\title{
Pyroglutamide-Based P2X7 Receptor Antagonists Targeting Inflammatory Bowel Disease
}

\section{Supporting information}

Germain Homerin $^{1,2}$, Samir Jawhara ${ }^{1, \S}$, Xavier Dezitter ${ }^{1,3,}$, Davy Baudelet ${ }^{1,2}$, Pierrick Dufrénoy ${ }^{1,2}$,

Benoît Rigo ${ }^{1,2}$, Régis Millet $^{1,3}$, Christophe Furman ${ }^{1,3}$, Guillaume Ragé $e^{1,3}$,Emmanuelle Lipka ${ }^{1,4}$, Amaury Farce ${ }^{1,3}$, Nicolas Renault ${ }^{1,3}$, Boualem Sendid ${ }^{1}$, Rogatien Charlet ${ }^{1}$, Jordan Leroy ${ }^{1}$, Melodie

Phanithavong ${ }^{5}$, Camille Richeval ${ }^{5}$, Jean-François Wiart ${ }^{5}$, Delphine Allorge ${ }^{5}$, Sahil Adriouch ${ }^{6,7}$,

Valérie Vouret-Craviari ${ }^{8,9}$, and Alina Ghinet ${ }^{1,2,10, *}$

${ }^{1}$ Inserm U995, LIRIC, Université de Lille, CHRU de Lille, Faculté de médecine - Pôle recherche, Place Verdun, F-59045 Lille Cedex, France

${ }^{2}$ Hautes Etudes d'Ingénieur (HEI), Yncréa Hauts-de-France, UCLille, Laboratoire de pharmacochimie, 13 rue de Toul, F-59046 Lille, France

${ }^{3}$ Institut de Chimie Pharmaceutique Albert Lespagnol, IFR114, 3 rue du Pr Laguesse, F-59006 Lille, France

${ }^{4}$ Faculté des Sciences Pharmaceutiques et Biologiques de Lille, Laboratoire de Chimie Analytique, F-59006 Lille Cedex, France

${ }^{5}$ CHRU de Lille, Centre de Biologie Pathologie, Laboratoire de Toxicologie \& Génopathies, Bd du Pr J. Leclercq, CS 70001, F-59037 Lille, France

${ }^{6}$ INSERM U905, F-76183 Rouen, France

${ }^{7}$ Normandie University, Institute for Research and Innovation in Biomedicine, F-76183 Rouen, France

${ }^{8}$ Institute for Research on Cancer and aging (IRCAN), F-06100 Nice, France

${ }^{9}$ University of Nice Cote d'Azur (UCA), F-06100 Nice, France

10 'Al. I. Cuza' University of Iasi, Faculty of Chemistry, Bd. Carol I, nr. 11, 700506 Iasi, Romania

Corresponding author: alina.ghinet@yncrea.fr 


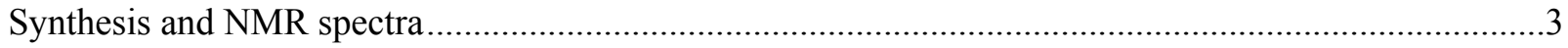

Chiral separation

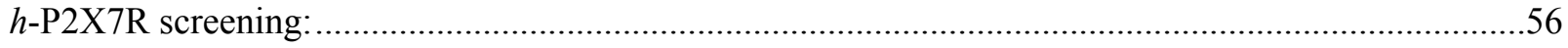

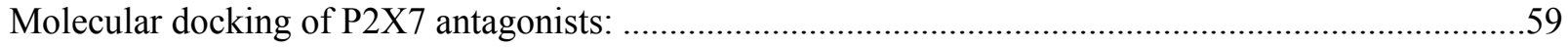

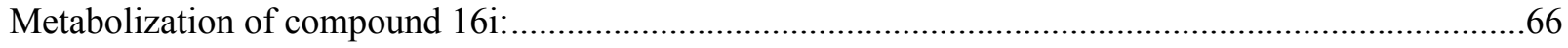

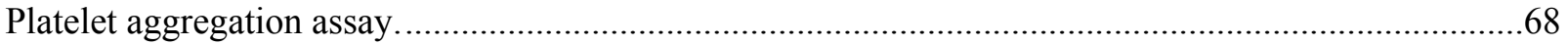

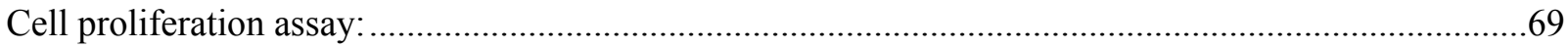

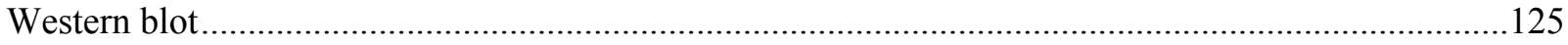

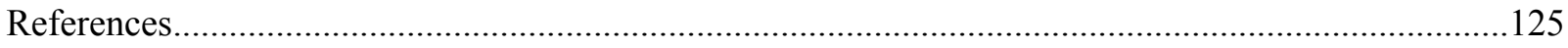




\section{Synthesis and NMR spectra}

Starting materials are commercially available and were used without further purification (suppliers: Carlo Erba Reagents S.A.S., Thermo Fisher Scientific Inc., and Sigma-Aldrich Co.). Intermediates were synthesized according to the methods described in the literature (references are given below, in figures S13 and S14, synthesis pathways are described in schemes S1-S6).

Nuclear magnetic resonance (NMR) spectra of unprecedented compounds are given below. They were acquired at $400 \mathrm{MHz}$ for ${ }^{1} \mathrm{H} \mathrm{NMR}$, at $100 \mathrm{MHz}$ for ${ }^{13} \mathrm{C} \mathrm{NMR}$, and at $376 \mathrm{MHz}$ for ${ }^{19} \mathrm{~F}$ NMR on a Varian 400-MR spectrometer with tetramethylsilane (TMS) as internal standard, at room temperature (RT).

\section{Chemistry.}

Esterification of PGA 2 was first conducted, leading to methyl pyroglutamate 5 (PGM) in quantitative yield. ${ }^{\text {la }}$ Synthesis of iminoether 6 and following rearrangement to methyl $N$-methyl pyroglutamate 7 was realized using dimethylsulfate. ${ }^{1 b}$ The $N$-methyl pyroglutamate 7 may be directly synthesized from methyl pyroglutamate $\mathbf{5}$ and methyl iodide also. The pathway involving iminoether $\mathbf{6}$ is however preferred for large-scale syntheses. Indeed, methyl iodide is toxic and very volatile while dimethyl sulfate is toxic and non-volatile. Further aminolysis of ester 7 with corresponding amines furnished series I of $N$-methyl PGAm 1a-d (Scheme S1).

Scheme S1. Synthesis of $N$-Methyl Pyroglutamide Derivatives 1a-d ${ }^{a}$ (Series I)

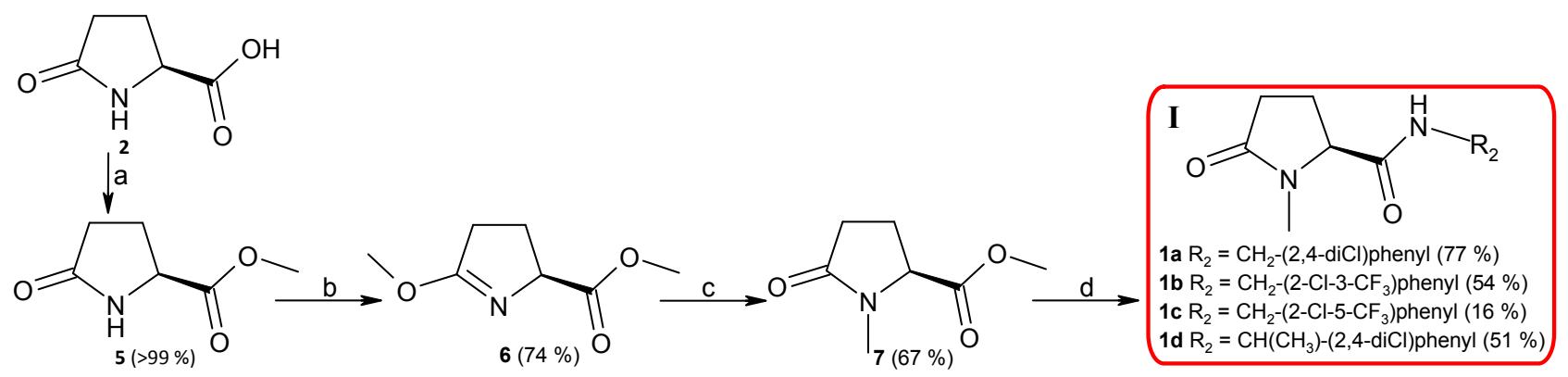

${ }^{a}$ Reagents and conditions. (a) $\mathrm{CH}_{3} \mathrm{SO}_{3} \mathrm{H}, \mathrm{MeOH} / \mathrm{CHCl}_{3}, \mathrm{MS} 3 \AA$, reflux, $24 \mathrm{~h}$, quantitative yield; (b) (i) $\mathrm{Me}_{2} \mathrm{SO}_{4}, 60^{\circ} \mathrm{C}, 12 \mathrm{~h}$; (ii) $\mathrm{Et}_{3} \mathrm{~N}, 0^{\circ} \mathrm{C}$-r.t., $1 \mathrm{~h}, 74 \%$; (c) $\mathrm{Me}_{2} \mathrm{SO}_{4}, \mathrm{THF}, 80^{\circ} \mathrm{C}, 4 \mathrm{~h}, 67 \%$; (d) amine, catalyst (PTSA or $\mathrm{ZrCl}_{4}$ ), r.t. $-120^{\circ} \mathrm{C}, 5-96 \mathrm{~h}, 16-77 \%$. 
$N$-aryl PGM 8a-l were next obtained by a copper initiated method we recently developed. ${ }^{\text {ic }}$ Aminolyses of esters 5, 7 and 8a-I furnished pyroglutamides 9-13, 15, 16a, 16e, 16g-o, 18 and 22. ${ }^{1 \mathrm{~d}}$ $N$-(hetero-)aryl derivatives 14, 16a-d, 16f, 16p-q, 17, 19-21 and 23 were obtained by copper initiated coupling from pyroglutamides $\mathbf{1 0 j}, \mathbf{1 1 e}, \mathbf{1 1 a}, \mathbf{1 1 g}-\mathbf{h}, \mathbf{1 2 b}$ and $\mathbf{1 3}$ (Scheme S2). ${ }^{1 \mathrm{c}}$

Scheme S2. Synthesis of $N$-Aryl Pyroglutamide Derivatives 14 to $\mathbf{2 3}^{a}$ (Series II) 


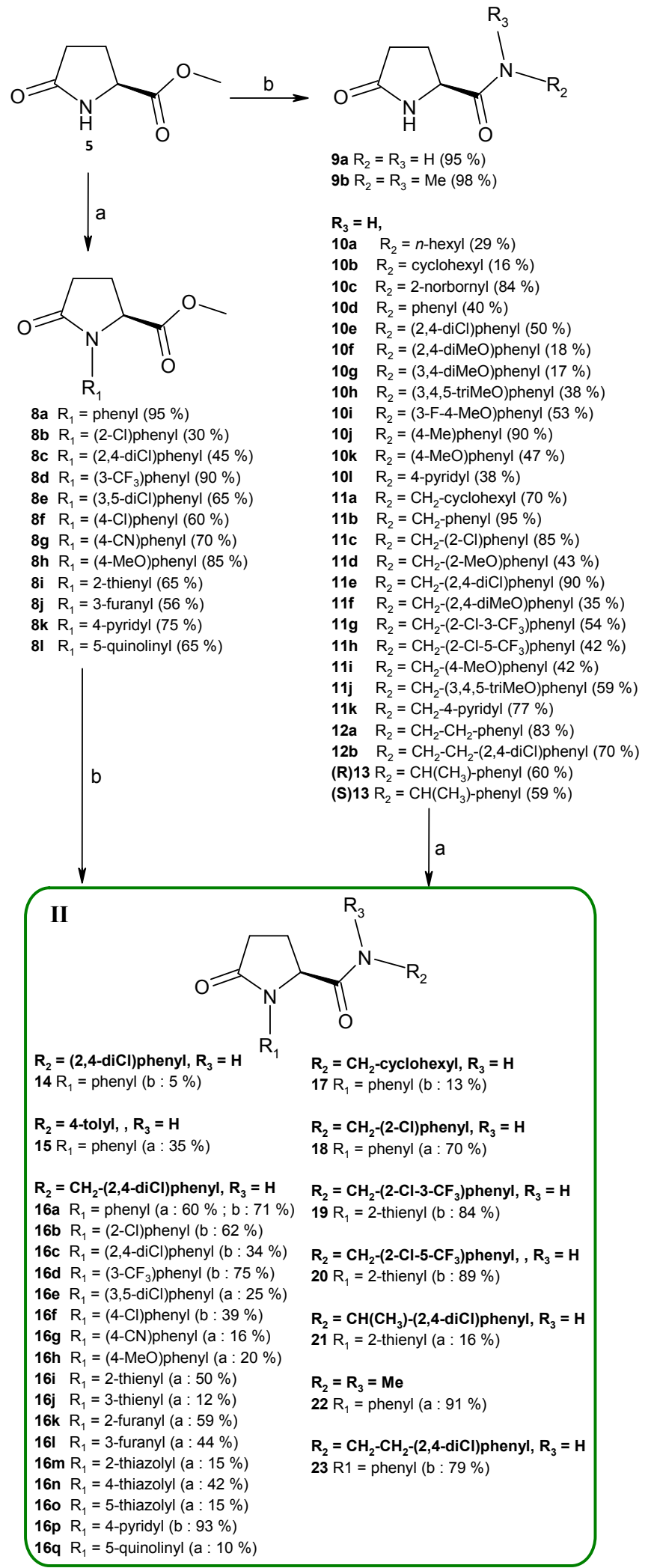

${ }^{a}$ Reagents and conditions. (a) $\mathrm{R}_{1}-\mathrm{X}(\mathrm{X}=\mathrm{Br}, \mathrm{I}), \mathrm{CuI}, N, N^{\prime}$-DMEDA, $\mathrm{Cs}_{2} \mathrm{CO}_{3}$, dioxane, $60^{\circ} \mathrm{C}-\mathrm{reflux}, 2-96 \mathrm{~h}, 12-$ $95 \%$; (b) amine, catalyst (PTSA or $\mathrm{ZrCl}_{4}$ ), r.t. $-120^{\circ} \mathrm{C}, 5-96 \mathrm{~h}, 5-98 \%$. 
PGM 5 was $N$-silylated to furnish compound $\mathbf{2 4},{ }^{1 \mathrm{f}}$ then reacted on the one hand with silylated

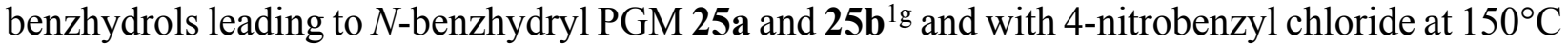
for $40 \mathrm{~h}$ to provide $N$-(4-nitrobenzyl) PGM 26. ${ }^{\text {ff }}$ Other $N$-benzyl PGM 27a, 27b and 27d-g were synthesized from Glu 3 according to our reductive amination method. ${ }^{\text {lh }}$ Ester $27 \mathrm{c}$ was synthesized from PGM 5 using $\mathrm{NaH}$ and 2,4-dichlorobenzyl chloride. ${ }^{1 \mathrm{i}}$ Amidation of esters 25-27 provided $\mathrm{N}$ benzyl PGAm 28-43 (Scheme S3). ${ }^{1 \mathrm{~d}}$

Scheme S3. Synthesis of $N$-Benzyl and $N$-Benzhydryl Pyroglutamide Derivatives 28 to $\mathbf{4 3}^{a}$ (Series III)

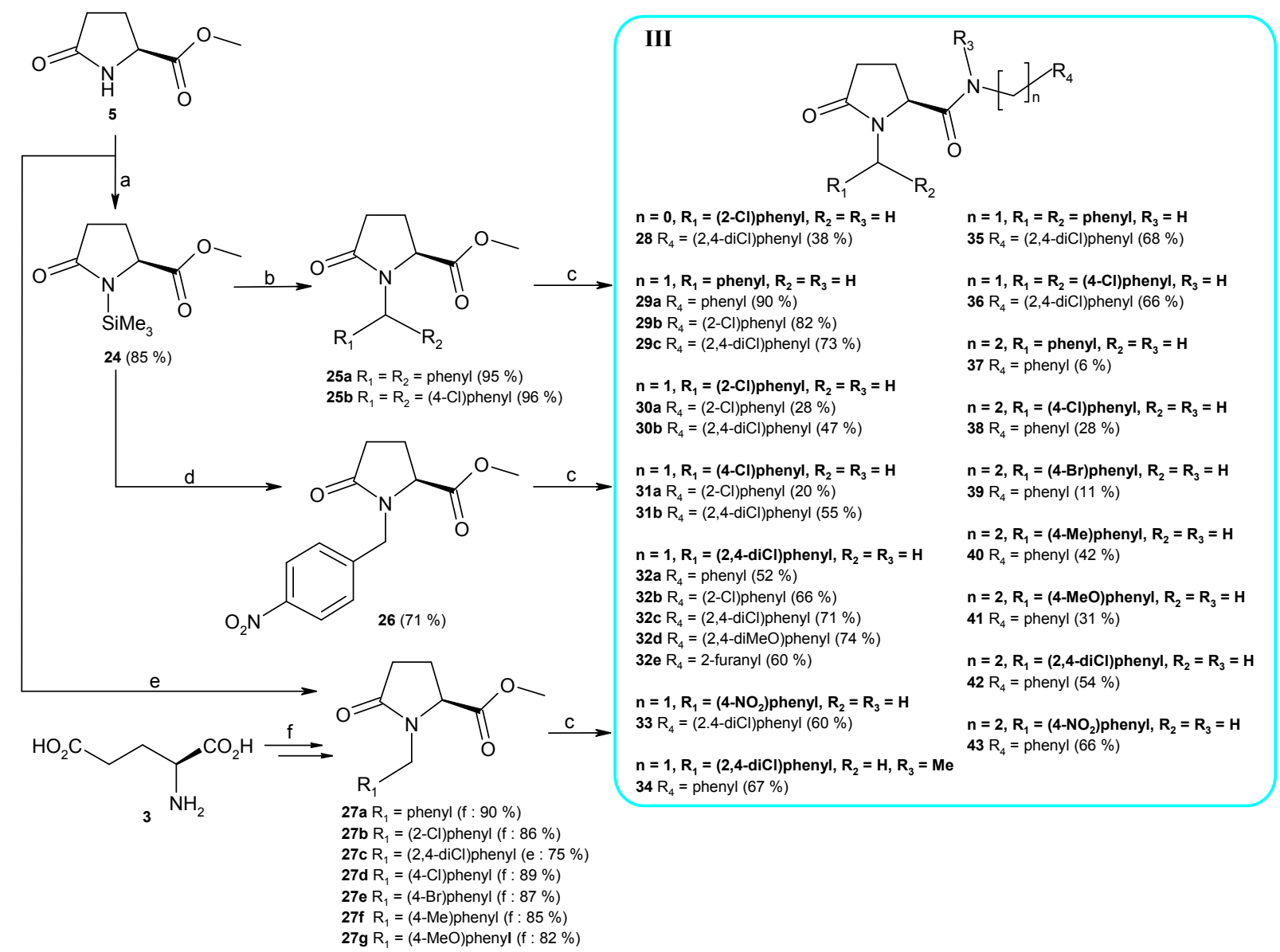

${ }^{a}$ Reagents and conditions. (a) $\mathrm{Me}_{3} \mathrm{SiCl}_{2} \mathrm{Et}_{3} \mathrm{~N}$, toluene, $80^{\circ} \mathrm{C}, 6 \mathrm{~h}, 85 \%$; (b) $\mathrm{Me}_{3} \mathrm{SiOCH}(\mathrm{Ph})_{2}, \mathrm{TfOH}, 130^{\circ} \mathrm{C}, 2-5 \mathrm{~h}$, 95-96\%; (c) amine, catalyst (PTSA or $\mathrm{ZrCl}_{4}$ ), r.t. $-120^{\circ} \mathrm{C}, 24-72 \mathrm{~h}, 6-90 \%$; (d) 4-nitrobenzyl chloride, $150^{\circ} \mathrm{C}, 40 \mathrm{~h}$, $71 \%$; (e) $\mathrm{ClCH}_{2}-(2,4-\mathrm{diCl}) \mathrm{Ph}, \mathrm{NaH}, \mathrm{THF}, 70^{\circ} \mathrm{C}, 24 \mathrm{~h}, 75 \%$; (f) (i) $\mathrm{Et}_{3} \mathrm{~N}, \mathrm{R}_{1}-\mathrm{CHO}, \mathrm{NaBH}_{4}, \mathrm{MeOH}, 2 \mathrm{~h}$; (ii) $\mathrm{EtOH}$, reflux, 5 h; (iii) $\mathrm{MeOH}, \mathrm{H}^{+}, 81-90 \%$. 
The introduction of a dimethylenamine moiety in position 3 of the lactam ring can be realized using Bredereck's reagent (BR). ${ }^{2}$ This transformation and further modifications of the obtained $\beta$ enaminones furnished compounds 44-48 constituting series IV (lactams modulated in position 3). Moreover, we have shown in two previous studies that BR was able to provide pyrroloimidazolediones 49 and $\mathbf{5 0}$ (compounds of series $\mathbf{V}$ ), which can be next processed to synthesize $\beta$-enaminones $\mathbf{5 1 - 5 4}$ (series IV) and pyrroloimidazoletriones $\mathbf{5 5}{ }^{1 \mathrm{j}, 1 \mathrm{k}}$ Thus, we enriched series IV with $\mathrm{NH}$-free lactams, and we obtained series $\mathbf{V}$ of pyrroloimidazoles (Scheme S4).

Interestingly, the Vilsmeier-Haak reaction (equivalent to Bredereck's reaction) on the substrate 11e, provided the $N$-formyl lactam 56, which was attached to series I for its bulkiness, while the electronic properties are strongly different (Scheme S4). ${ }^{1 \mathrm{j}}$ 
Scheme S4. Synthesis of Pyroglutamide Derivatives 44 to 55 (Series IV and V) and Pyroglutamide Derivatives $\mathbf{5 6}^{a}$ (Series I)

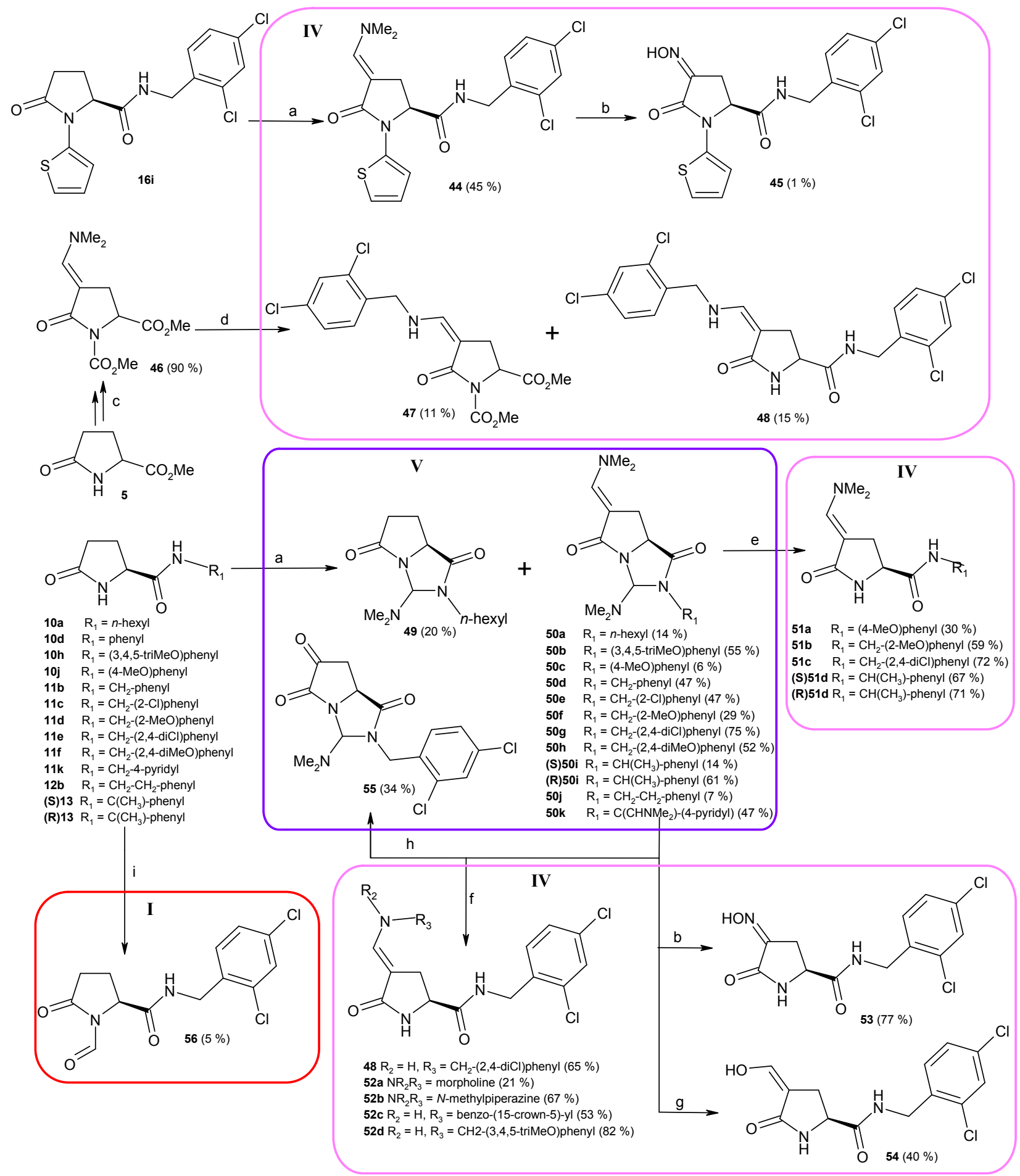

${ }^{a}$ Reagents and conditions. (a) $t$ - $\mathrm{BuOCH}\left(\mathrm{NMe}_{2}\right)_{2}, 120^{\circ} \mathrm{C}, 1-24 \mathrm{~h}, 7-75 \%$; (b) $\mathrm{NaNO}_{2}, \mathrm{CH}_{3} \mathrm{CO}_{2} \mathrm{H}$, THF, $0^{\circ} \mathrm{C}, 3 \mathrm{~h}, 1-77 \%$; (c) (1) $\mathrm{ClCO}_{2} \mathrm{Me}, \mathrm{Et}_{3} \mathrm{~N}, \mathrm{DMF},-10^{\circ} \mathrm{C}, 1 \mathrm{~h}, 33 \%$; (2) $t$-BuOCH$\left(\mathrm{NMe}_{2}\right)_{2}, 120^{\circ} \mathrm{C}, 2 \mathrm{~h}, 90 \%$; (d) amine, $\mathrm{ZrCl}_{4}, 60^{\circ} \mathrm{C}, 48 \mathrm{~h}, 11-15 \%$; (e) $\mathrm{MeOH}$, reflux, $24 \mathrm{~h}, 30-72 \%$; (f) amine, toluene, reflux, $24 \mathrm{~h}$, 21-82\%; (g) $\mathrm{CH}_{3} \mathrm{SO}_{3} \mathrm{H}, \mathrm{H}_{2} \mathrm{O}$, reflux, $24 \mathrm{~h}, 40 \%$; (h) $\mathrm{NaIO}_{4}, \mathrm{EtOAc} / \mathrm{CH}_{3} \mathrm{CN} / \mathrm{H}_{2} \mathrm{O}$, r.t., 1 h, 34\%; (i) $\left(\mathrm{Me}_{2} \mathrm{~N}^{+}=\mathrm{CHCl}, \mathrm{Cl}^{-}\right)$, DMF, r.t., 48 h, $5 \%$. 
Oxazolidinone 57 was synthesized from Ser $\mathbf{4}$ according the method reported in the literature. ${ }^{3}$ Methyl ester 58 was further obtained upon esterification of oxazolidinone $\mathbf{5 7},{ }^{\text {la }}$ and then treated with 2,4-dichlorobenzylamine to provide the amide 59. ${ }^{1 \mathrm{~d}}$ Afterward, the 2-iodothiophene was reacted with the resulting amide under copper-coupling conditions and provided $\mathrm{N}$ (thienyl)oxazolidinone $\mathbf{6 0}$ and side products (Scheme S5). ${ }^{1 \mathrm{c}}$

To introduce the amide linker in position 4 of the lactam, a synthetic strategy starting from biosourced itaconic acid $\mathbf{6 1}$ was privileged. Further esterification to dimethyl itaconate $\mathbf{6 2}$ and cyclisation using ammonia provided lactam $63 .{ }^{4}$ Aminolysis and copper-coupling conditions were then applied to lactam 63 and provided PGAm 64 and 65.

Some other analogues of the lactam moiety were investigated, such as pyridines $\mathbf{6 7}$ and $\mathbf{6 8}$, synthesized through previously described route. The extension of the pyrrolidone ring to piperidone was also realized. Compound $\mathbf{7 0}$ was obtained by peptide coupling, and used as the substrate of BR to furnish $\beta$-enaminone 71 . 
Scheme S5. Synthesis of Pyroglutamide Analogues 59, 60, 64, 65, 67, 68, 70 and $71^{a}$ (Series VI)
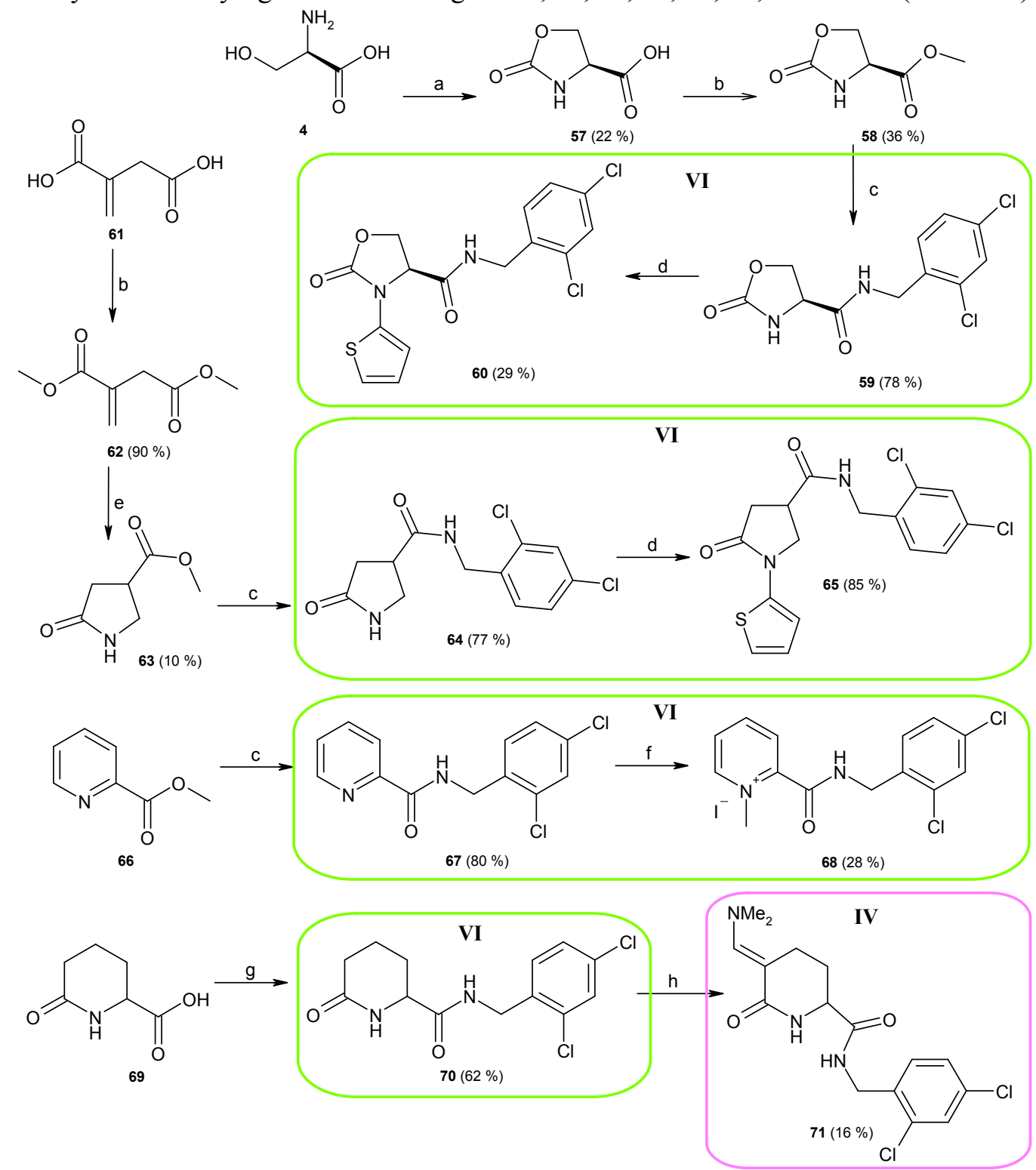

${ }^{a}$ Reagents and conditions. (a) $\mathrm{ClCO}_{2} \mathrm{Ph}, \mathrm{NaOH}$, toluene $/ \mathrm{H}_{2} \mathrm{O},<30^{\circ} \mathrm{C}, 3 \mathrm{~h}, 22 \%$; (b) $\mathrm{MeOH}, \mathrm{SOCl}_{2}$, reflux, $48 \mathrm{~h}, 36-$ 90\%; (c) 2,4-dichlorobenzylamine, PTSA, r.t., 24 h, 77-80\%; (d) 2-iodothiophene, CuI, $N, N^{\prime}$-DMEDA, $\mathrm{Cs}_{2} \mathrm{CO}_{3}$, dioxane, r.t., 24 h, 25-85\%; (e) $\mathrm{NH}_{3}, \mathrm{H}_{2} \mathrm{O}$, rt, 10\%; (f) MeI, reflux, 24 h, 28\%; (g) 2,4-dichlorobenzylamine, EDCI, $\mathrm{CH}_{2} \mathrm{Cl}_{2}$, r.t., $24 \mathrm{~h}, 62 \%$; (h) $t$ - $\mathrm{BuOCH}\left(\mathrm{NMe}_{2}\right)_{2}, 120^{\circ} \mathrm{C}, 3 \mathrm{~h}, 16 \%$.

In order to evaluate the affinity of our antagonists with the receptor, a radioligand was synthesized from aminoquinoleine $\mathbf{7 2}$. Thiocyanate $\mathbf{7 3}$ and aminoquinoleines $\mathbf{7 4}$ were synthesized according to a method of the literature (Scheme S6). ${ }^{5}$ The tritiation of compound $\mathbf{7 4 b}$ was outsourced to provide the radiolabeled P2X7-ligand 75, which was further used as the reference in the competitive binding assay further described. 
Scheme S6. Synthesis of P2X7 ligands 74 and $\mathbf{7 5}^{a}$

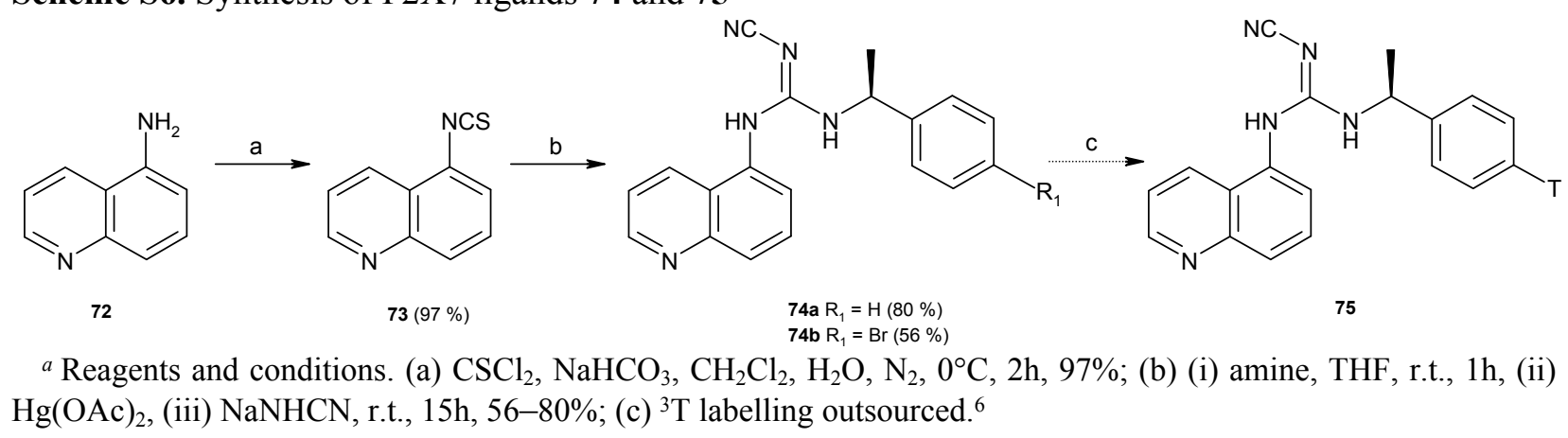

Because harsh chemical conditions were used for aminolyses, compound 16i was selected for supercritical fluid chiral (SFC) chromatography (Figure S15-2 of the Supporting Information) to verify the retention of configuration. Only one isomer was identified, confirming the retention of configuration along the syntheses previously described..$^{1 \mathrm{c}, 1 \mathrm{~d}, 1 \mathrm{j}, 1 \mathrm{k}}$

The synthesis or racemate of compound 16i has been also realized starting from methyl DLpyroglutamate. The racemate has also been analyzed by SFC and resulted in the presence of two enantiomers (Figure S15-1 of the Supporting Information). 
$\overbrace{N_{O}^{N}}^{H}>_{R_{2}}^{H}$

1a $\mathrm{R}_{2}=\mathrm{CH}_{2}$-(2,4-diCl)phenyl (ref 7)

$1 b \mathrm{R}_{2}=\mathrm{CH}_{2}-\left(2-\mathrm{Cl}-3-\mathrm{CF}_{3}\right)$ phenyl (ref 8)

1d $\mathrm{R}_{2}=\mathrm{CH}\left(\mathrm{CH}_{3}\right)-(2,4-$ dicl)phenyl (ref 9)<smiles>COC(=O)C1CCC(OC)=N1</smiles><smiles>[Y6]N1C(=O)CCC1C(=O)OC</smiles><smiles>[R]N1C(=O)CCC1C(=O)OC</smiles>

8a $R_{1}=$ phenyl (ref 13)

8b $R_{1}=(2-C l)$ phenyl (ref 13)

8c $R_{1}=(2,4-d i C l) p h e n y l$ (ref 13)

8d $\mathrm{R}_{1}=\left(3-\mathrm{CF}_{3}\right)$ phenyl (ref 13)

$8 e R_{1}=(3,5$-diCl)phenyl (ref 13)

8f $R_{1}=(4-C l)$ phenyl (ref 13)

$8 g R_{1}=(4-C N)$ phenyl (ref 13)

8 h $\mathrm{R}_{1}=(4-\mathrm{MeO})$ phenyl (ref 13)

8i $R_{1}=2$-thienyl (ref 13)

8j $R_{1}=3$-furanyl (ref 13)

$8 \mathbf{k} R_{1}=4$-pyridyl (ref 13)

$81 R_{1}=5$-quinolinyl (ref 13)

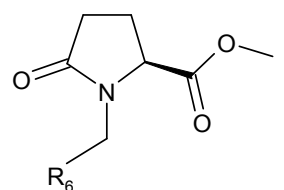

27a $R_{1}=$ phenyl (ref 22)

$27 b R_{1}=(2-C l)$ phenyl (ref 22)

$27 \mathrm{c} \mathrm{R} \mathrm{R}_{1}=(2,4$-diCl)phenyl (ref 22)

27d $\mathrm{R}_{1}=(4-\mathrm{Cl})$ phenyl (ref 22)

$27 e R_{6}=(4-B r)$ phenyl (ref 22)

$27 f R_{6}=(4-M e)$ phenyl (ref 22)

$27 \mathrm{~g} \mathrm{R}_{6}=(4-\mathrm{MeO})$ phenyl $($ ref 22$)$

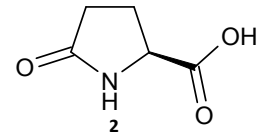

$\mathrm{HO}_{2} \mathrm{C}$

3<smiles>N[C](CO)C(=O)O</smiles><smiles>COC(=O)C1CCC(=O)N1</smiles><smiles>[R]N([R])C(=O)C1CCC(=O)N1</smiles>

9a $R_{2}=R_{3}=H$ (ref 13)

$9 b R_{2}=R_{3}=M e($ ref 13)

$\mathrm{R}_{3}=\mathrm{H}$,

10a $R_{2}=n$-hexyl (ref 14)

10b $R_{2}=$ cyclohexyl (ref 15)

10c $R_{2}=2$-norbornyl (ref 16)

10d $R_{2}=$ phenyl (ref 17)

10e $R_{2}=(2,4-d i C l)$ phenyl (ref 9)

$10 f \quad R_{2}=(2,4-$ diMeO)phenyl (ref 9)

10h $R_{2}=(3,4,5$-triMeO)phenyl (ref 16)

10j $R_{2}=(4-M e)$ phenyl (ref 13)

10k $\mathrm{R}_{2}=$ (4-MeO)phenyl (ref 18)

10I $\mathrm{R}_{2}=4$-pyridyl (ref 16)

11a $\mathrm{R}_{2}=\mathrm{CH}_{2}$-cyclohexyl (ref 9)

11b $\mathrm{R}_{2}=\mathrm{CH}_{2}$-phenyl (ref 19)

11c $\mathrm{R}_{2}=\mathrm{CH}_{2}-(2-\mathrm{Cl})$ phenyl (ref 20)

11d $\mathrm{R}_{2}=\mathrm{CH}_{2}-(2-\mathrm{MeO}$ )phenyl (ref 21)

11e $\mathrm{R}_{2}=\mathrm{CH}_{2}$-(2,4-diCl)phenyl ( $r$ ef 9 )

11f $\mathrm{R}_{2}=\mathrm{CH}_{2}$-(2,4-diMeO)phenyl (ref 9)

$11 \mathbf{k} \mathrm{R}_{2}=\mathrm{CH}_{2}-4$-pyridyl (ref 9)

12a $\mathrm{R}_{2}=\mathrm{CH}_{2}-\mathrm{CH}_{2}$-phenyl (ref 13)

12b $\mathrm{R}_{2}=\mathrm{CH}_{2}-\mathrm{CH}_{2}$-(2,4-diCl)phenyl (ref 16)

(R)13 $\mathrm{R}_{2}=\mathrm{C}\left(\mathrm{CH}_{3}\right)$-phenyl (ref 19)

(S) $13 \mathrm{R}_{2}=\mathrm{C}\left(\mathrm{CH}_{3}\right)$-phenyl (ref 19)<smiles>[R]C([R])N1C(=O)CCC1C(=O)N([R3])CC([R])(C)C</smiles>

$\mathrm{n}=\mathbf{0}, \mathrm{R}_{\mathbf{6}}=(\mathbf{2}-\mathrm{Cl})$ phenyl, $\mathrm{R}_{7}=\mathrm{H}, \mathrm{R}_{3}=\mathrm{H}$

$28 \mathrm{R}_{4}=(2,4$-diCl)phenyl (ref 9)

$\mathrm{n}=\mathbf{1}, \mathrm{R}_{\mathbf{6}}=$ phenyl, $\mathrm{R}_{7}=\mathrm{H}, \mathrm{R}_{3}=\mathrm{H}$

29a $R_{4}=$ phenyl (ref 9 )

29b $\mathrm{R}_{4}=(2-\mathrm{Cl})$ phenyl (ref 9)

29c $R_{4}=(2,4-d i C l)$ phenyl (ref 9)

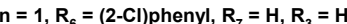

30b $\mathrm{R}_{4}=(2,4-\mathrm{diCl})$ phenyl (ref 9)

$\mathrm{n}=1, \mathrm{R}_{6}=\left(4-\mathrm{NO}_{2}\right)$ phenyl, $\mathrm{R}_{7}=\mathrm{H}, \mathrm{R}_{3}=\mathrm{H}$

$33 \mathrm{R}_{4}=(2,4-$ diCl)phenyl (ref 9)

$\mathrm{n}=1, \mathrm{R}_{6}=\mathrm{R}_{\mathbf{7}}=$ phenyl, $\mathrm{R}_{3}=\mathrm{H}$

$35 \mathrm{R}_{4}=(2,4-\mathrm{diCl})$ phenyl (ref 9)

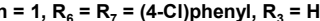

$36 R_{4}=(2,4-$ diCl)phenyl (ref 9)<smiles>[R]N([R])C(=O)C1CCC(=O)N1[R]</smiles>

$R_{\mathbf{2}}=$ 4-toluyl, , $R_{3}=\mathbf{H}$

$15 \mathrm{R}_{1}=$ phenyl (ref 13)

$\mathbf{R}_{\mathbf{2}}=\mathrm{CH}_{\mathbf{2}}$-(2,4-diCl)phenyl, $\mathrm{R}_{3}=\mathbf{H}$

16a $R_{1}=$ phenyl (ref 9)

16b $R_{1}=(2-C l)$ phenyl (ref 9)

16d $R_{1}=\left(3-C_{3}\right)$ phenyl (ref 9)

16e $R_{1}=(3,5-d i C l)$ phenyl (ref 13)

16i $R_{1}=2$-thienyl (ref 16)

16p $R_{1}=4$-pyridyl (ref 9)

16q $R_{1}=5$-quinolinyl (ref 9)

$\mathbf{R}_{\mathbf{2}}=\mathrm{CH}_{2}-(\mathbf{2}-\mathrm{Cl})$ phenyl, $\mathbf{R}_{3}=\mathrm{H}$

$18 \mathrm{R}_{1}=$ phenyl (ref 13)

$\mathbf{R}_{\mathbf{2}}=\mathbf{R}_{\mathbf{3}}=\mathbf{M e}$

$22 R_{1}=$ phenyl $(\operatorname{ref} 13)$

\author{
6)
}

26 (ref 22)<smiles>COC(=O)C1CCC(=O)N1S(C)(=O)=O</smiles>

24 (ref 22)

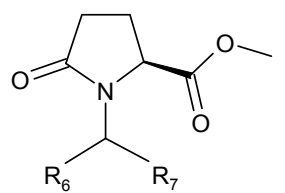

25a $R_{6}=R_{7}=$ phenyl (ref 23)

25b $R_{6}=R_{7}=(4-C l)$ phenyl (ref 23)<smiles>C/C=C(/S)N1C(=O)/C(=C/N(C)C)CC1C(=O)NCc1ccc(Cl)cc1Cl</smiles><smiles>O=C1NC(C(=O)NCc2ccc(Cl)cc2Cl)C/C1=C/NCc1ccc(Cl)cc1Cl</smiles>

Figure S1. Reagents and Products Synthesized as Described in the Literature (1). References for the synthesis of pyroglutamic acid derivatives are given at the last page of this document. 
<smiles>[R7]N1C(=O)C2CCC(=O)N2C1[14CH3]</smiles><smiles>[R10]N1C(=O)C2C/C(=C\N(C)C)C(=O)N2C1NC</smiles>

50a $\quad \mathrm{R}_{1}=n$-hexyl $(\operatorname{ref} 16)$

50b $R_{1}=(3,4,5$-triMeO)phenyl (ref 16)

50c $R_{1}=(4-M e O)$ phenyl (ref 16$)$

50d $\mathrm{R}_{1}=\mathrm{CH}_{2}$-phenyl (ref 16)

50e $\quad \mathrm{R}_{1}=\mathrm{CH}_{2}-(2-\mathrm{Cl})$ phenyl (ref 16)

50f $\quad \mathrm{R}_{1}=\mathrm{CH}_{2}-(2-\mathrm{MeO})$ phenyl (ref 16)

50g $\mathrm{R}_{1}=\mathrm{CH}_{2}-(2,4-$ diCl)phenyl (ref 16)

50h $\mathrm{R}_{1}=\mathrm{CH}_{2}$-(2,4-diMeO)phenyl (ref 16)

(S)50i $\mathrm{R}_{1}=\mathrm{CH}\left(\mathrm{CH}_{3}\right)$-phenyl (ref 16)

(R)50i $\mathrm{R}_{1}=\mathrm{CH}\left(\mathrm{CH}_{3}\right)$-phenyl (ref 25)

50j $\quad \mathrm{R}_{1}=\mathrm{CH}_{2}-\mathrm{CH}_{2}$-phenyl (ref 16)

50k $\quad \mathrm{R}_{1}=\mathrm{C}\left(\mathrm{CHNMe}_{2}\right)-(4-$ pyridyl) $($ ref 25$)$<smiles>O=C1CC(C(=O)NCc2ccc(Cl)cc2Cl)NC1=O</smiles>

$53($ ref 16)<smiles>O=CN1C(=O)CCC1C(=O)NCc1ccc(Cl)cc1Cl</smiles><smiles>O=C1NC(C(=O)O)CO1</smiles><smiles>COC(=O)C1COC(=O)N1</smiles>

58 (ref 26)<smiles></smiles><smiles>[R]N([R])/C=C1\CC(C(=O)NCc2ccc(Cl)cc2Cl)NC1=O</smiles>

52a $\mathrm{NR}_{2} \mathrm{R}_{3}=$ morpholine (ref 16) 52b NR $\mathrm{R}_{3}=\mathrm{N}$-methylpiperazine (ref 16) 52c $R_{2}=H, R_{3}=$ benzo-(15-crown-5)-yl (ref 16) 52d R $\mathrm{R}_{2}=\mathrm{H}, \mathrm{R}_{3}=\mathrm{CH}_{2}-(3,4,5$-triMeO)phenyl (ref 16) 51c $\mathrm{R}_{1}=\mathrm{CH}_{2}$-(2,4-diCl)phenyl (ref 16 (S)51d R $\mathrm{R}_{1}=\mathrm{CH}\left(\mathrm{CH}_{3}\right)$-phenyl (ref 25) (R)51d R $=\mathrm{CH}\left(\mathrm{CH}_{3}\right)$-phenyl (ref 25)<smiles>CNC1N(Cc2ccc(Cl)cc2Cl)C(=O)C2CC(=O)C(=O)N21</smiles><smiles>C=C(CC(=O)Cl)C(=O)O</smiles>

61<smiles>C=C(CC(=O)OC)C(=O)OC</smiles>

62
63 (ref 27)<smiles>CN(C)/C=C1/CCC(C(=O)NCc2ccc(Cl)cc2Cl)NC1=O</smiles><smiles>COC(=O)c1ccccn1</smiles>

66<smiles>O=C1CCCC(C(=O)O)N1</smiles>

69<smiles>O=C1CCCC(C(=O)NCc2ccc(Cl)cc2Cl)N1</smiles>

70 (ref 16)<smiles>Nc1cccc2ncccc12</smiles><smiles>O=S(=O)(O)c1cccc2ncccc12</smiles><smiles>C[C@H](N/C(=N/C#N)Nc1cccc2ncccc12)c1ccc(Br)cc1</smiles><smiles>CC(N/C(=N/C#N)Nc1cccc2ncccc12)c1ccc(F)cc1</smiles>

Figure S2. Reagents and Products Synthesized as Described in the Literature (2). References for the synthesis of pyroglutamic acid derivatives and other compounds are given at the last page of this document. 
(S)- $N$-[2-Chloro-5-(trifluoromethyl)benzyl]-1-methyl-5-oxopyrrolidine-2-carboxamide (1c).

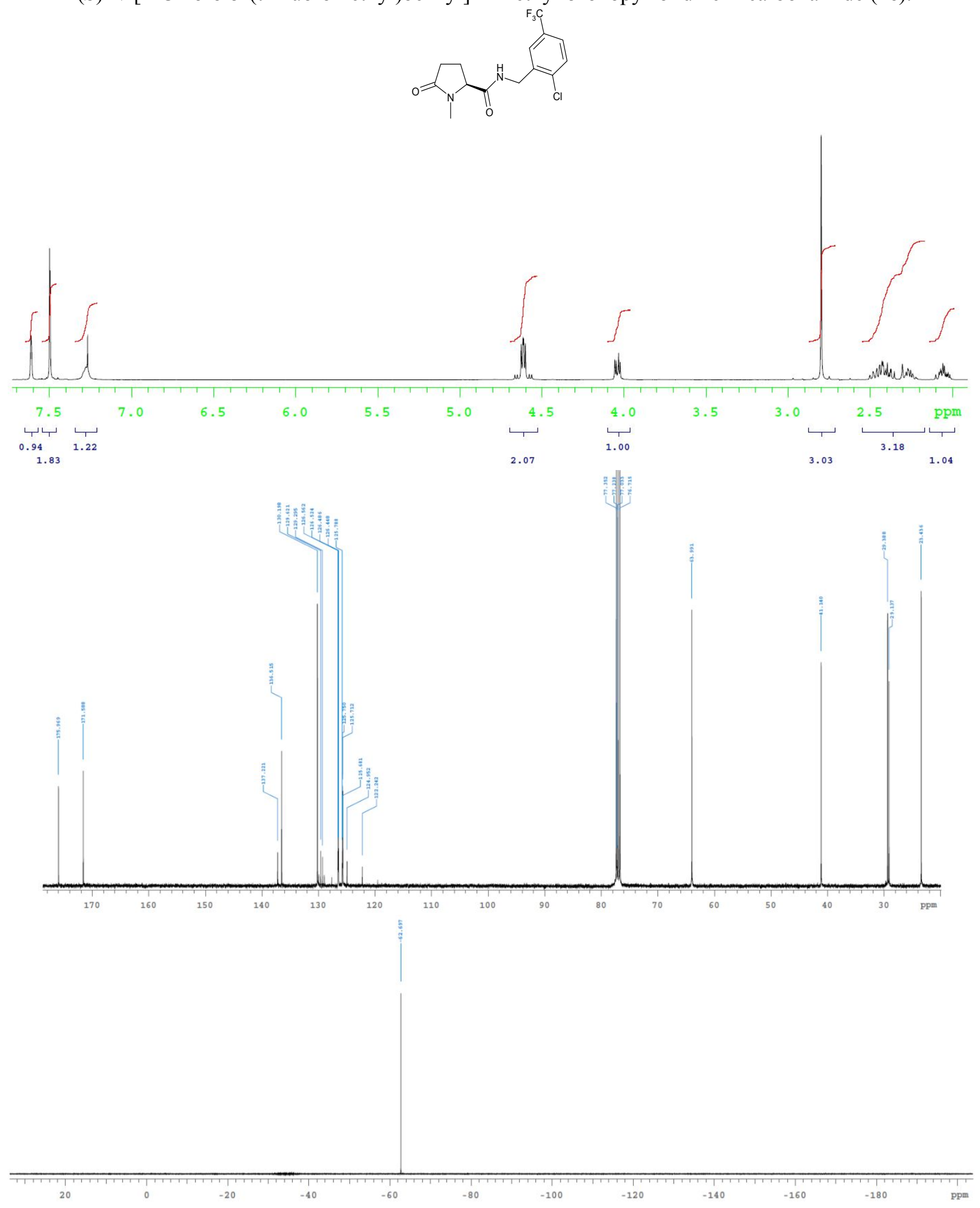


(S)-N-(3,4-Dimethoxyphenyl)-5-oxopyrrolidine-2-carboxamide (10g).

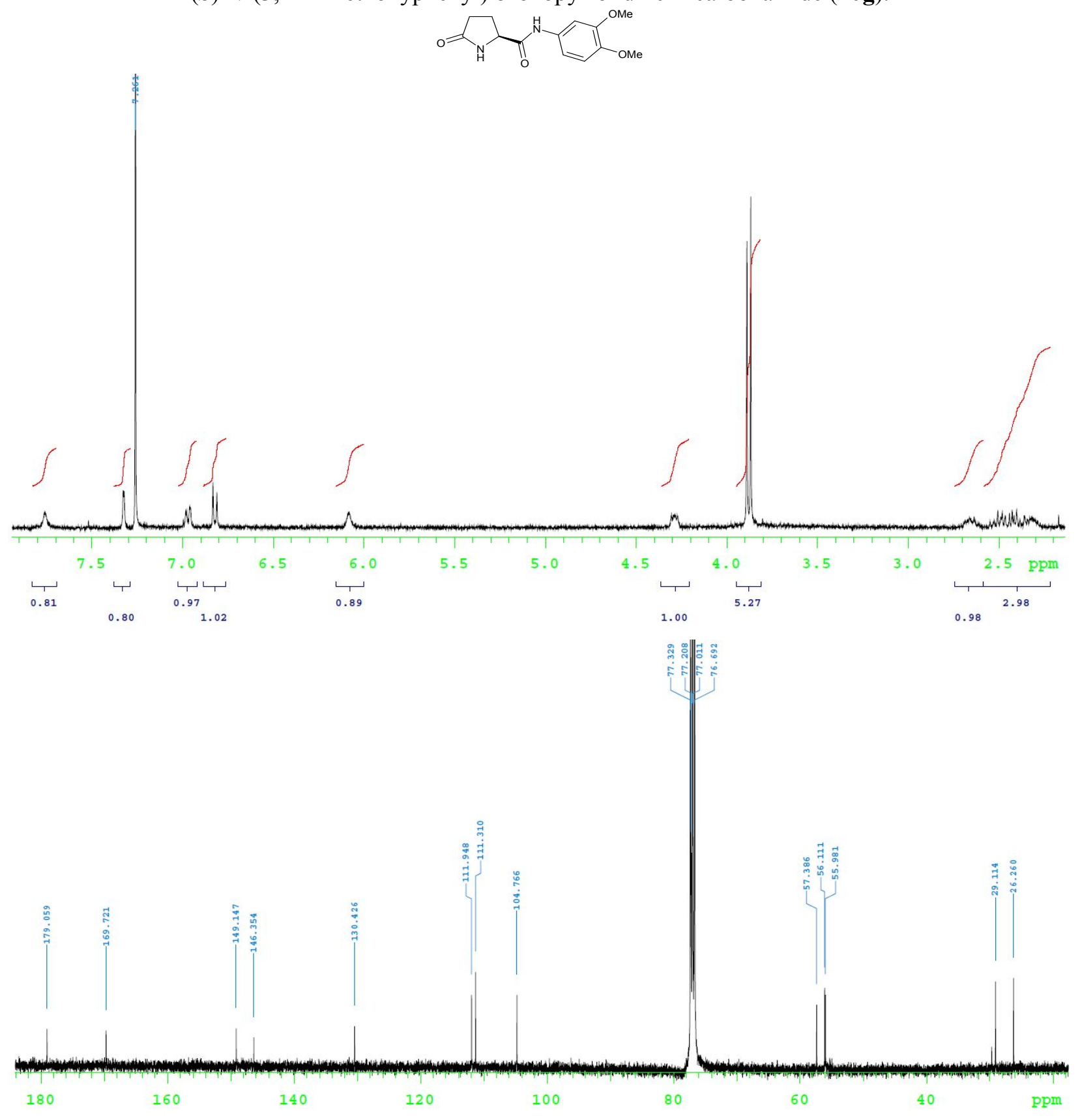


(S)-N-(3-Fluoro-4-methoxyphenyl)-5-oxopyrrolidine-2-carboxamide (10i).
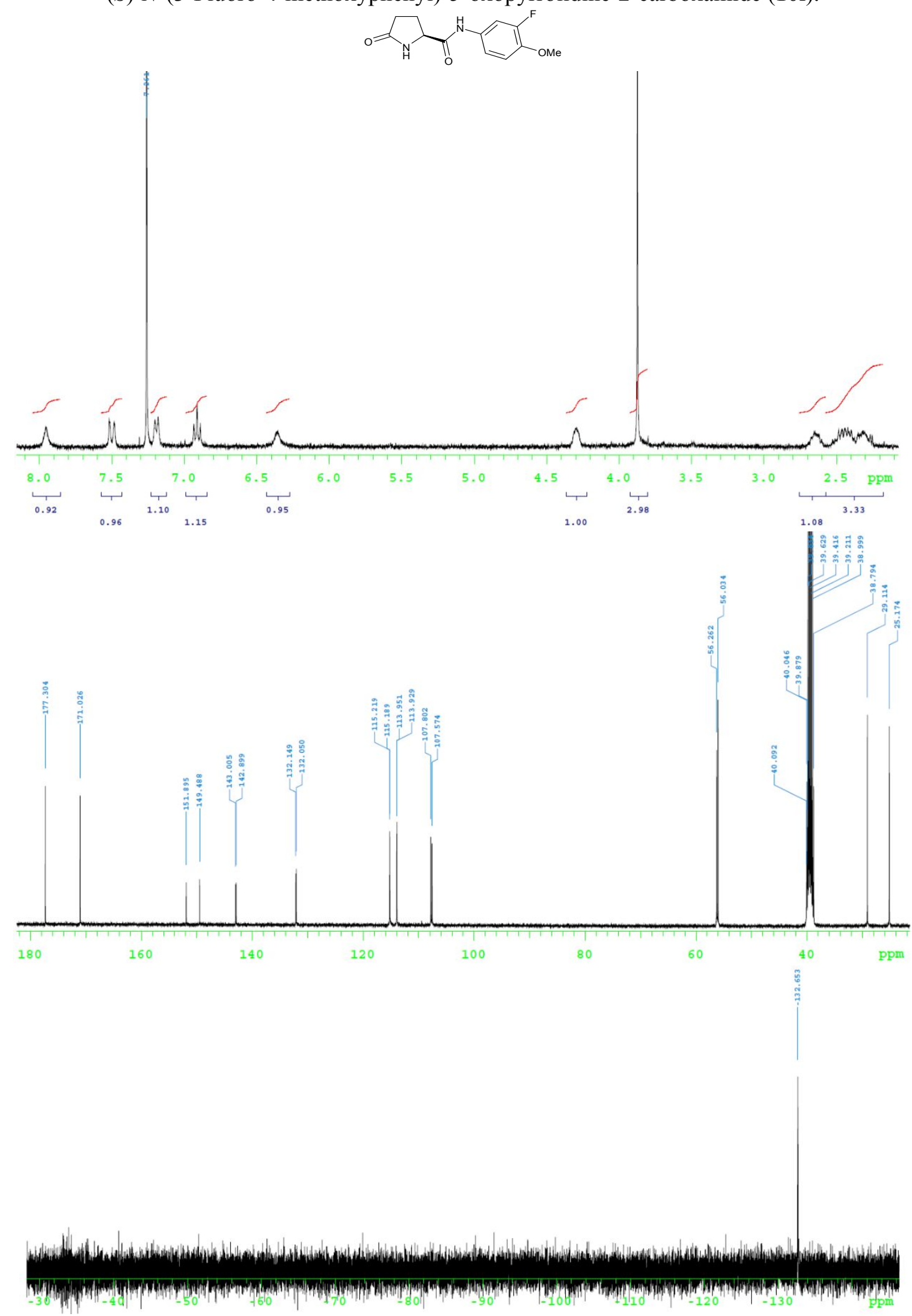
(S)-N-(2-Chloro-3-(trifluoromethyl)benzyl)-5-oxopyrrolidine-2-carboxamide (11g).

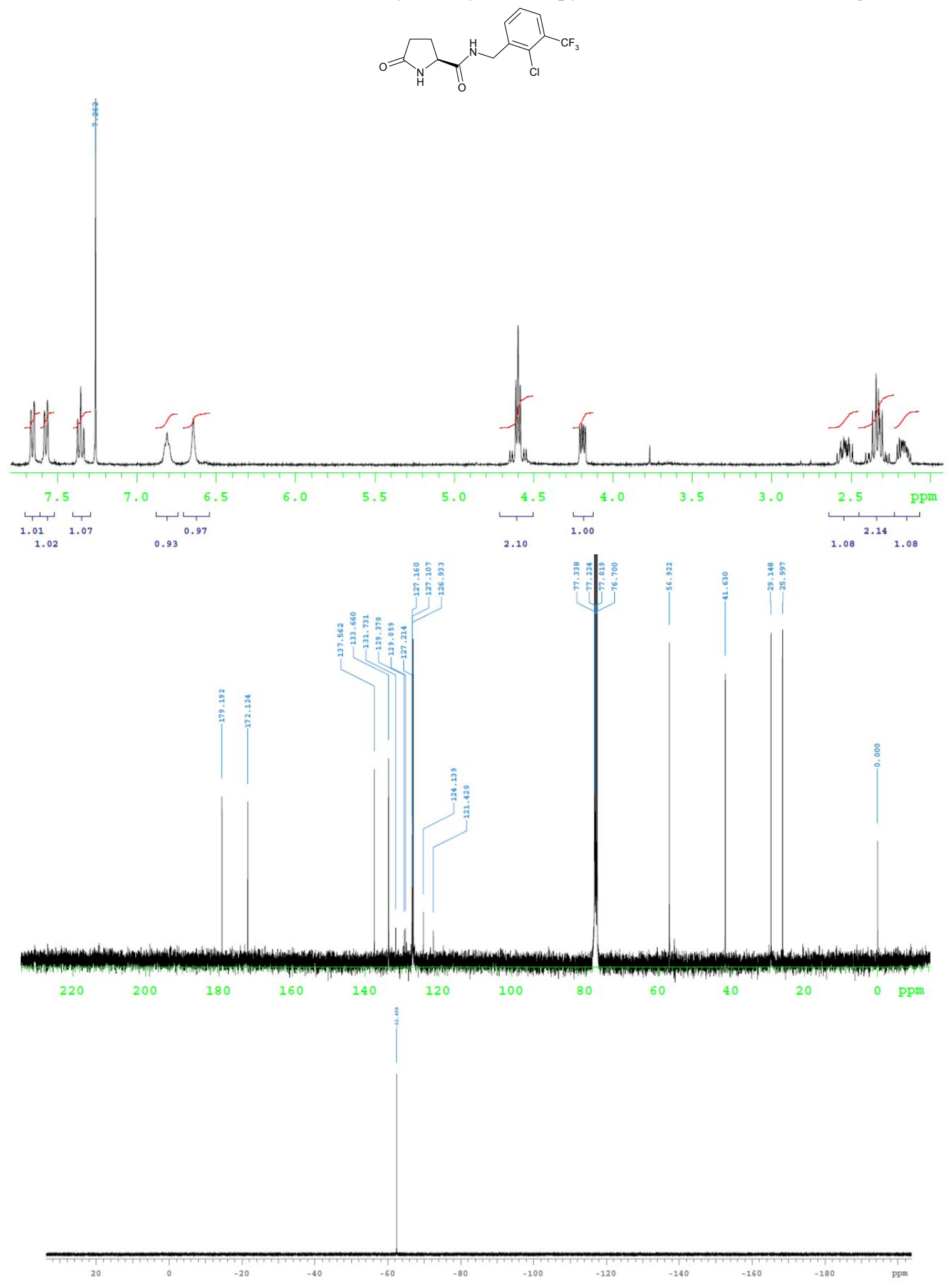


(S)- $N$-(2-Chloro-5-(trifluoromethyl)benzyl)-5-oxopyrrolidine-2-carboxamide (11h).

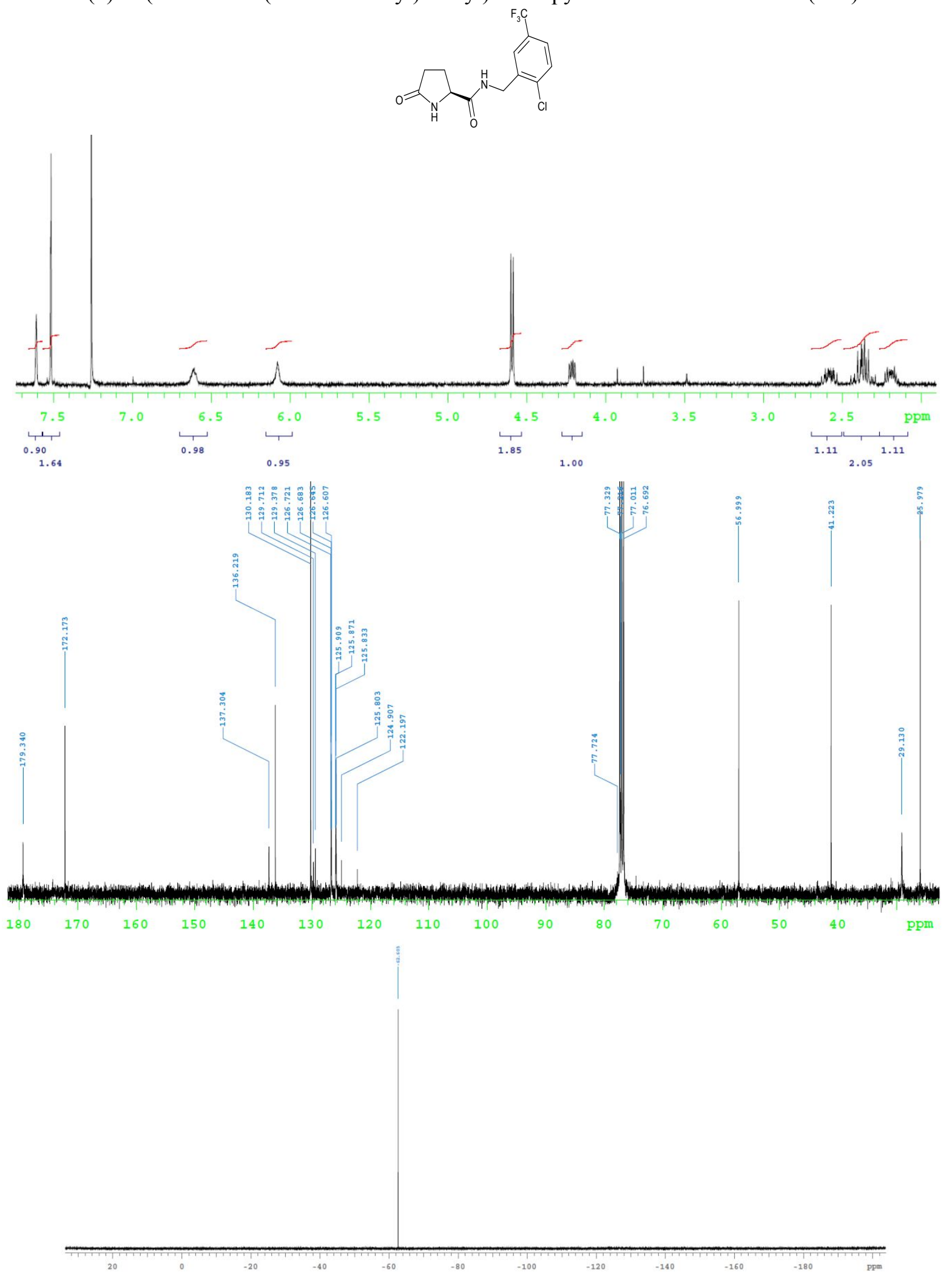


(S)-N-(4-Methoxybenzyl)-5-oxopyrrolidine-2-carboxamide (11i).
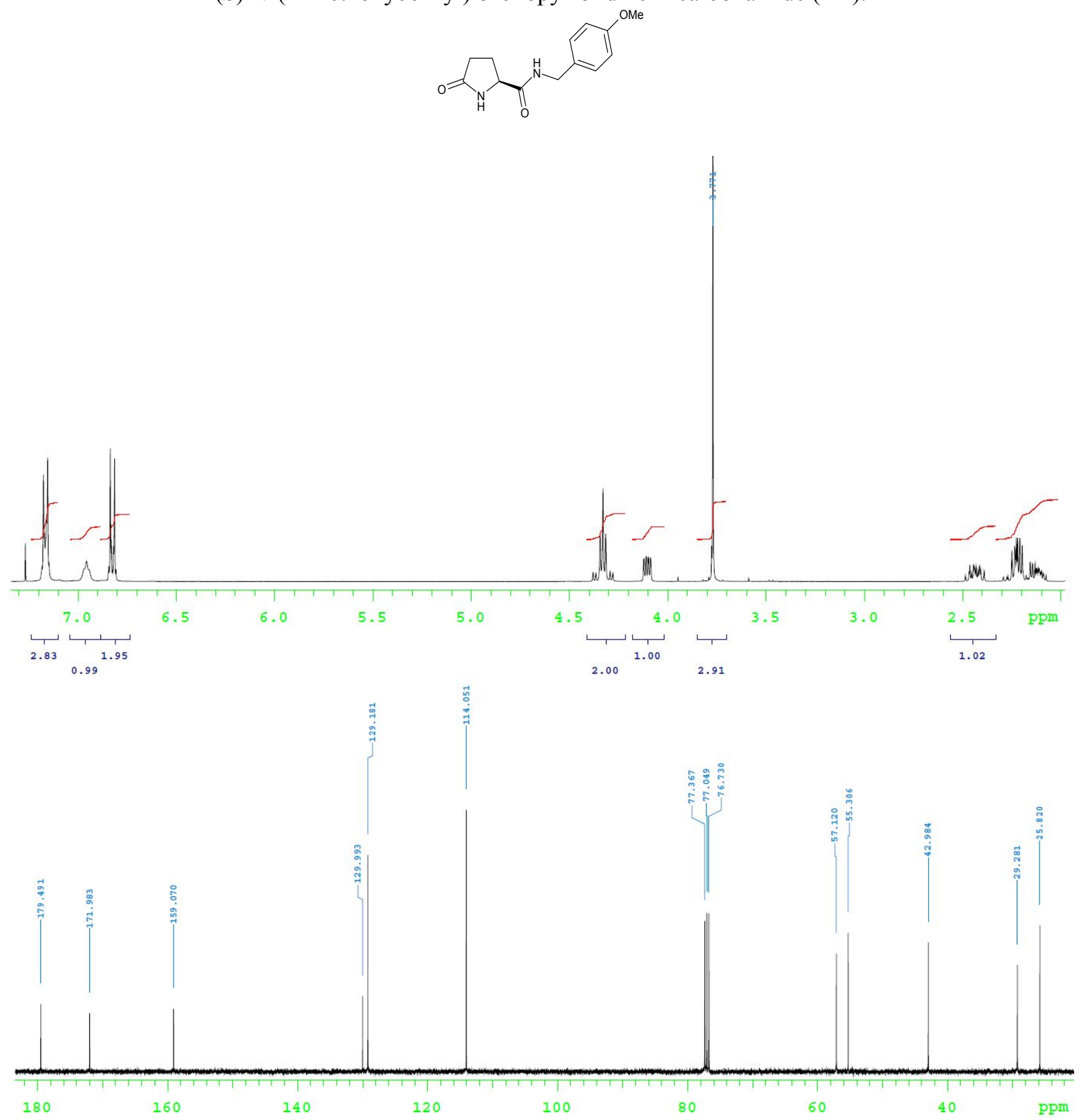
(S)-5-Oxo- $N$-(3,4,5-trimethoxybenzyl)pyrrolidine-2-carboxamide (11j).

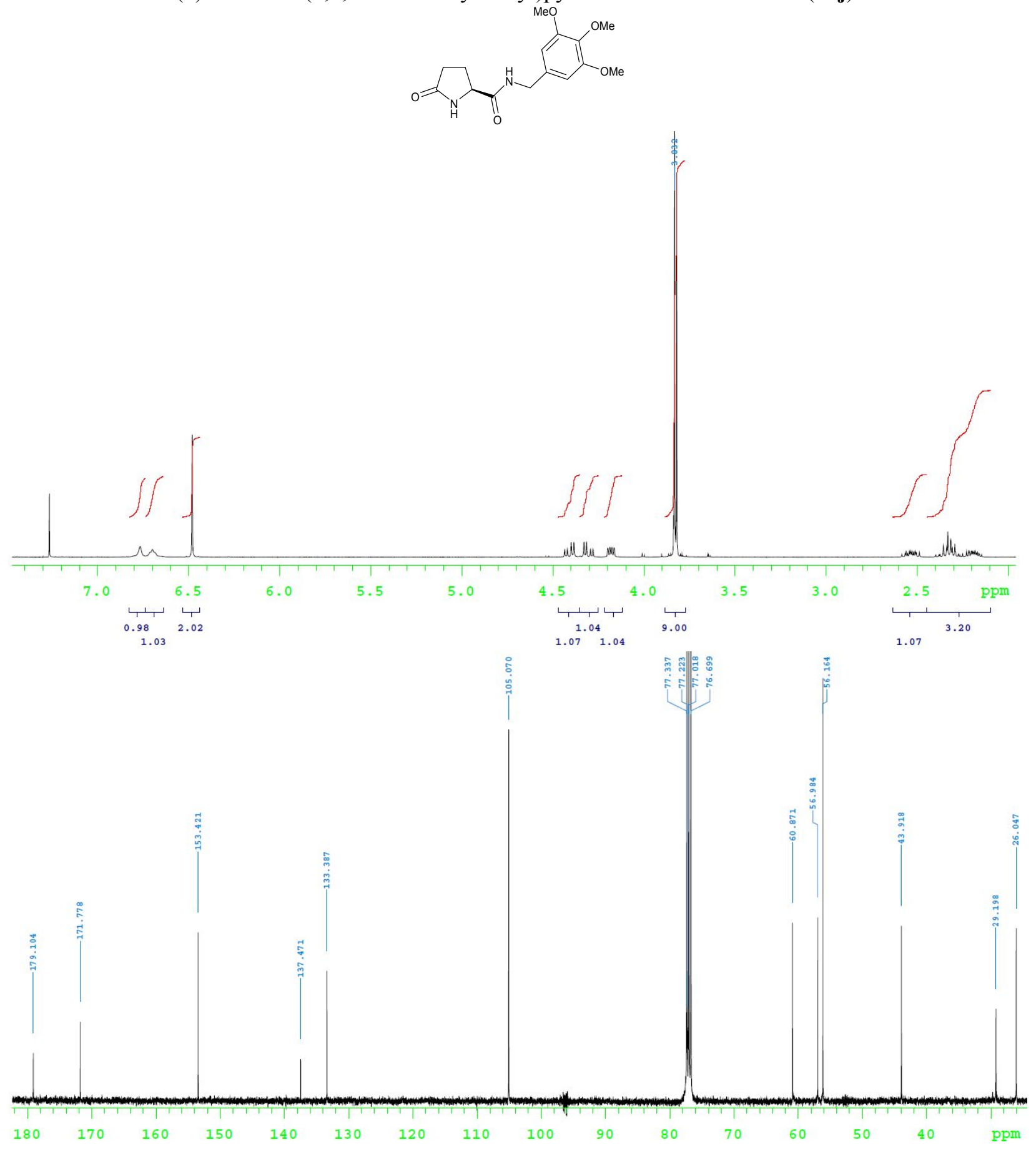


(S)-N-(2,4-Dichlorophenyl)-5-oxo-1-phenylpyrrolidine-2-carboxamide (14).
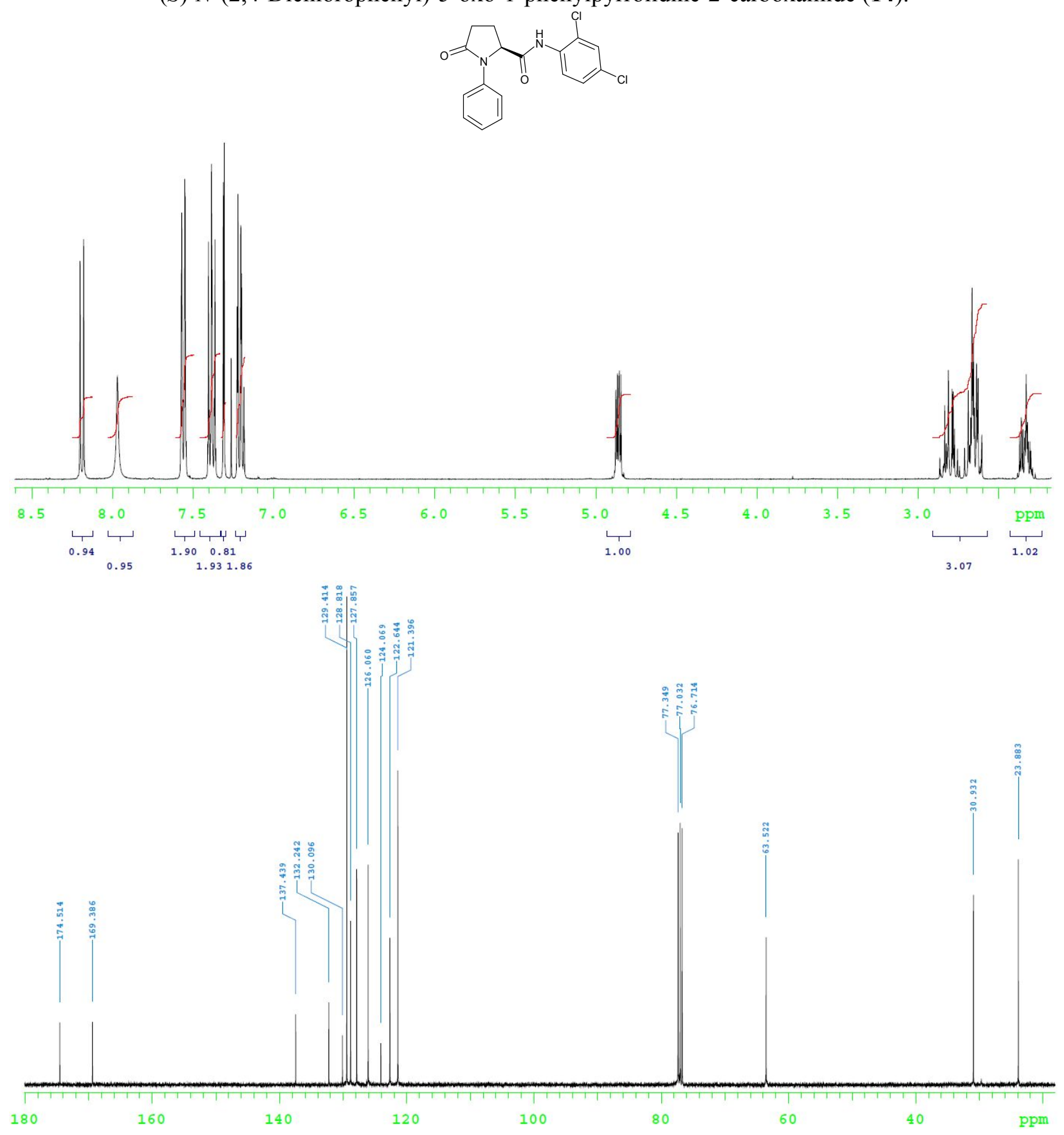
S22 
(S)-N-(2,4-Dichlorobenzyl)-5-oxo-1-(1,3-thiazol-2-yl)pyrrolidine-2-carboxamide (16m).

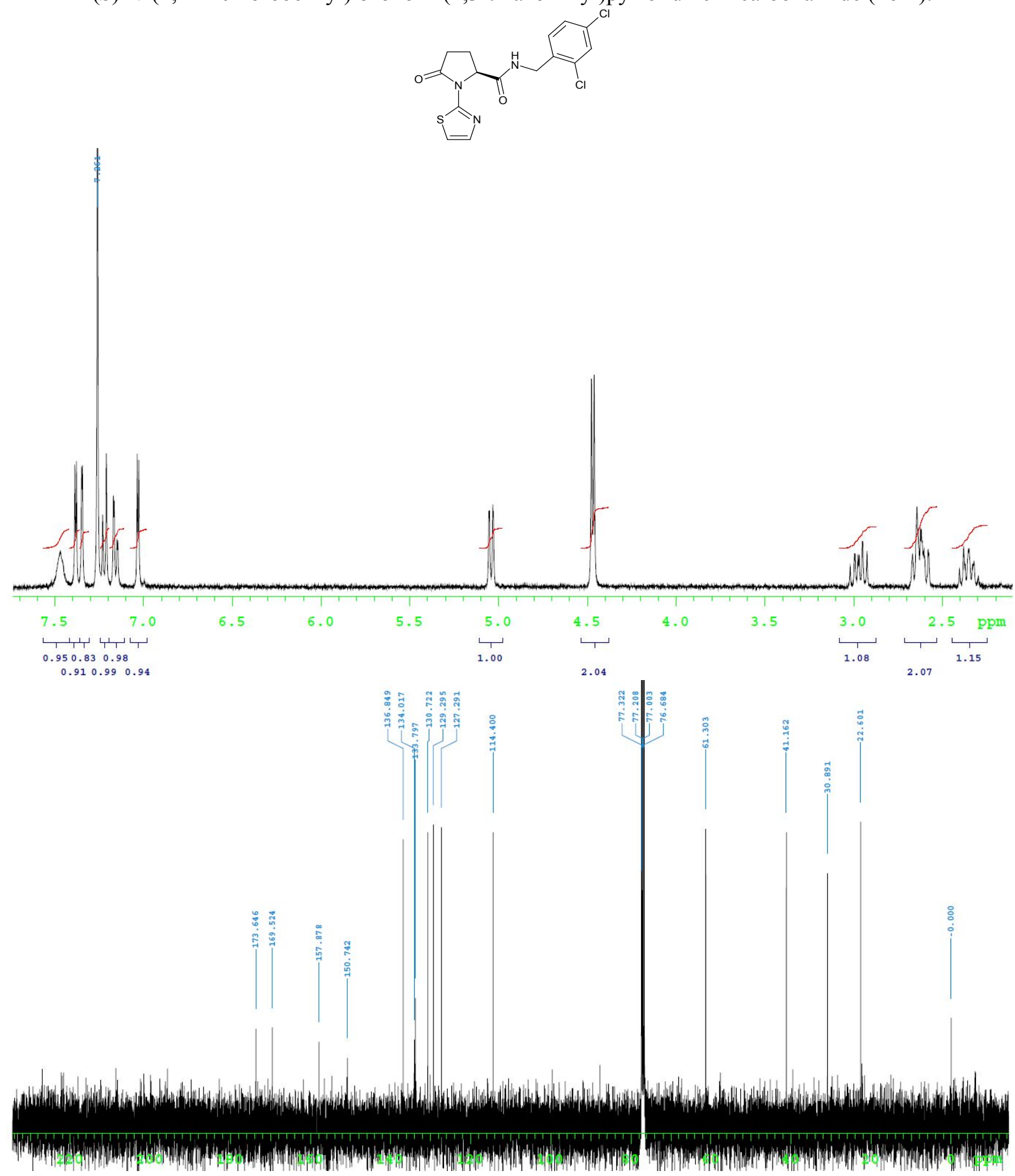


(S)-N-(2,4-Dichlorobenzyl)-5-oxo-1-(1,3-thiazol-4-yl)pyrrolidine-2-carboxamide (16n).

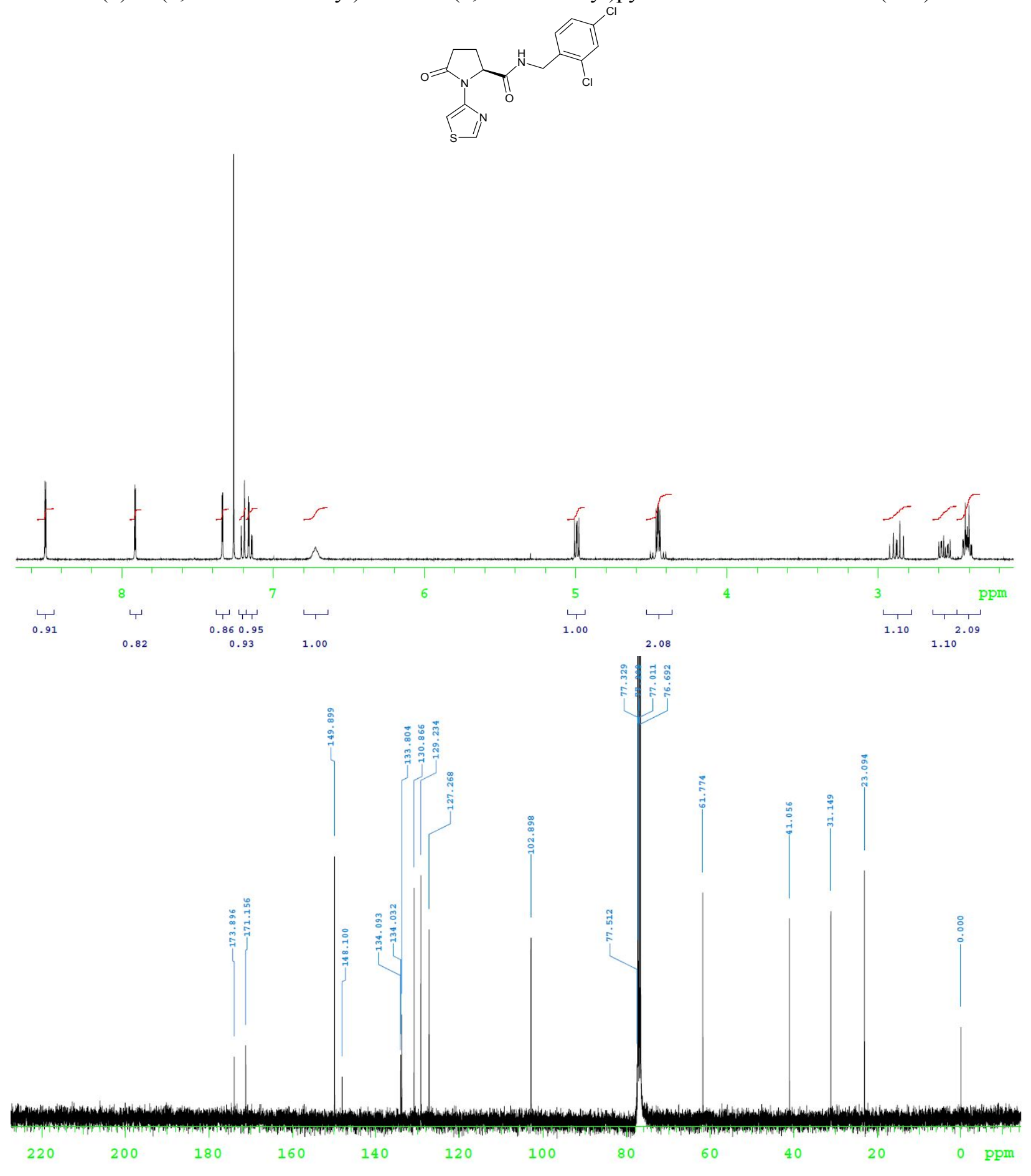


(S)-N-(2,4-Dichlorobenzyl)-5-oxo-1-(1,3-thiazol-5-yl)pyrrolidine-2-carboxamide (16o).

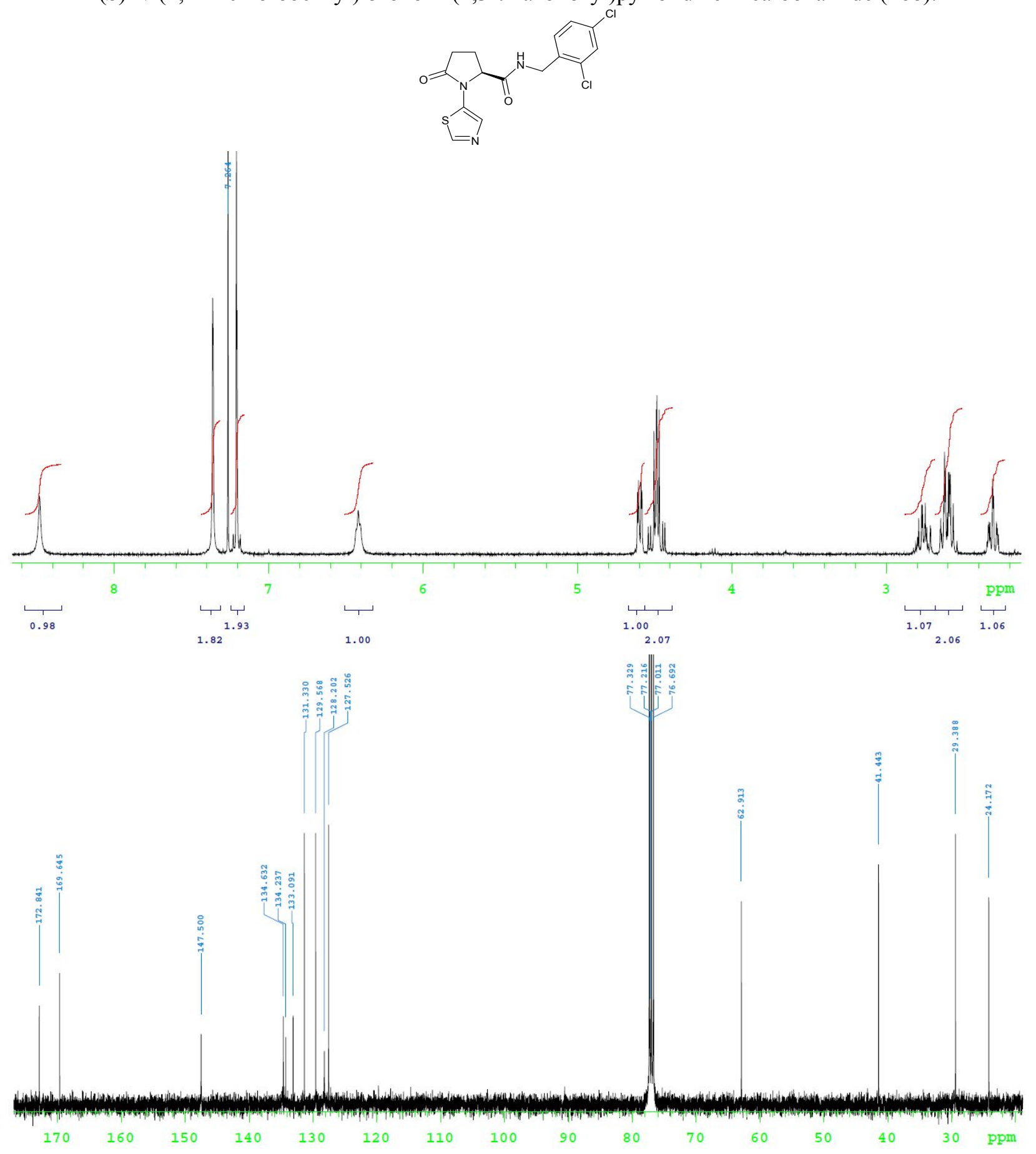


(S)-N-(Cyclohexylmethyl)-5-oxo-1-phenylpyrrolidine-2-carboxamide (17).
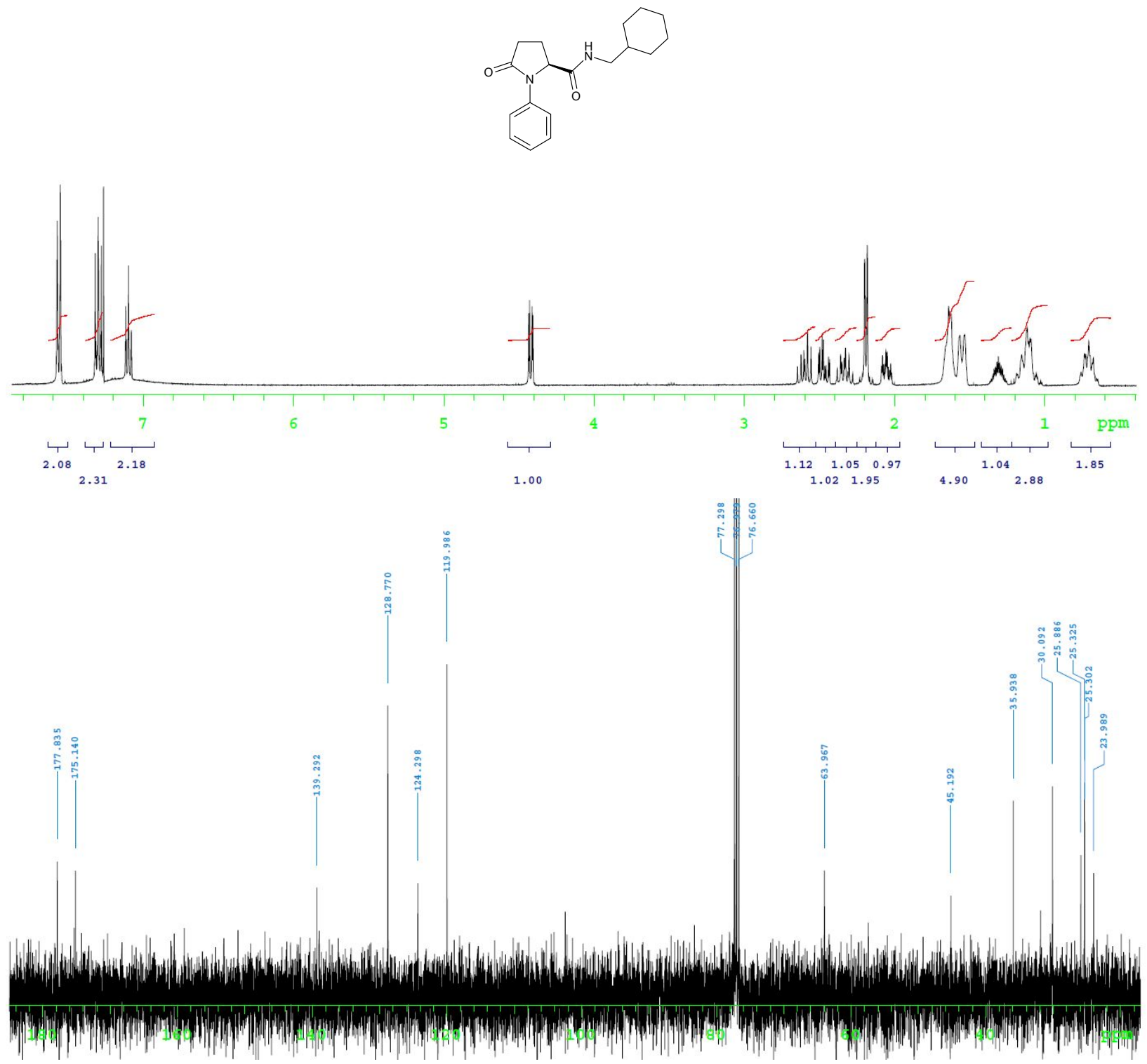

S26 
(S)-N-[2-Chloro-3-(trifluoromethyl)benzyl]-5-oxo-1-(thiophen-2-yl)pyrrolidine-2-carboxamide (19).

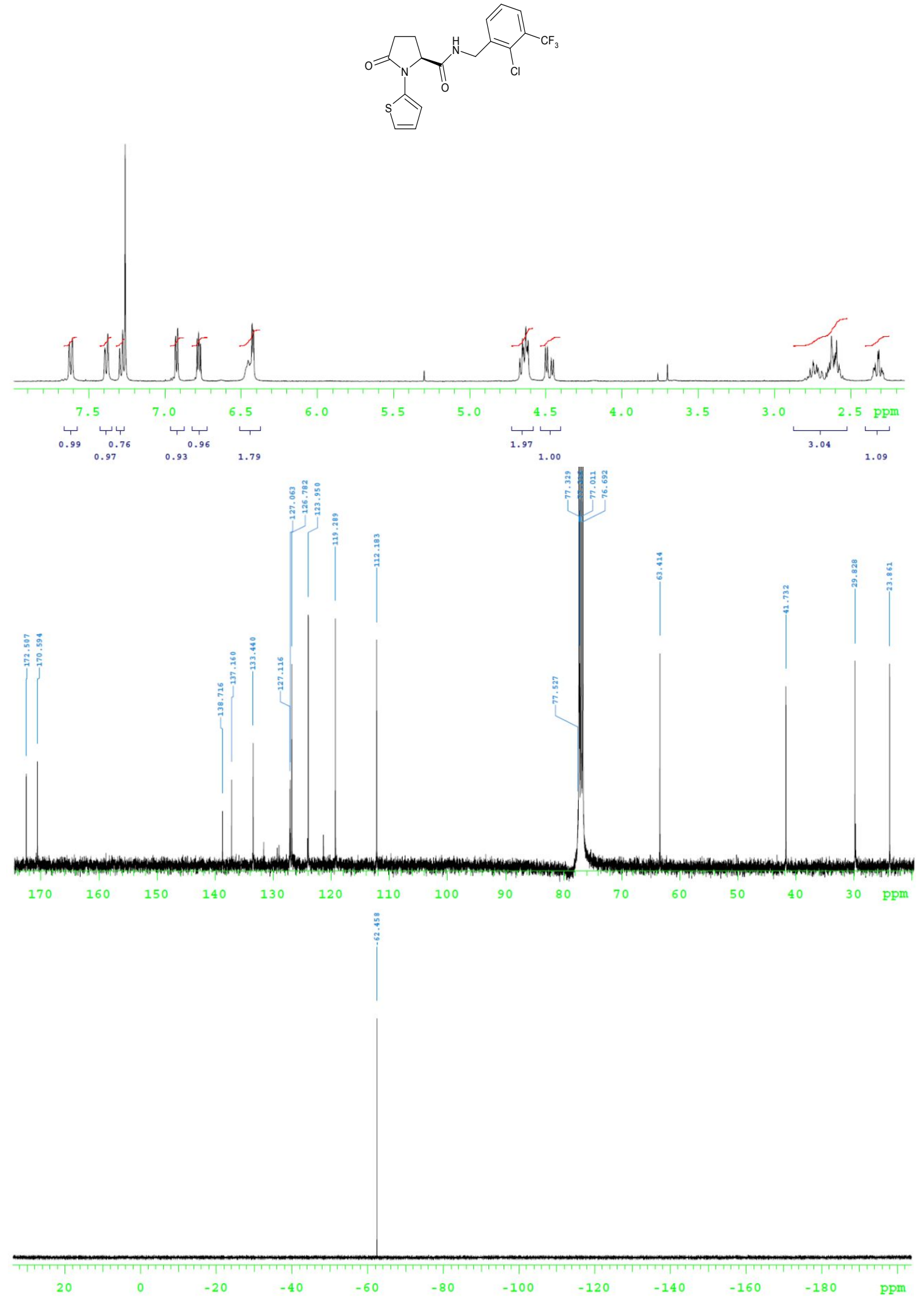


(S)-N-[2-Chloro-5-(trifluoromethyl)benzyl]-5-oxo-1-(thiophen-2-yl)pyrrolidine-2-carboxamide (20).
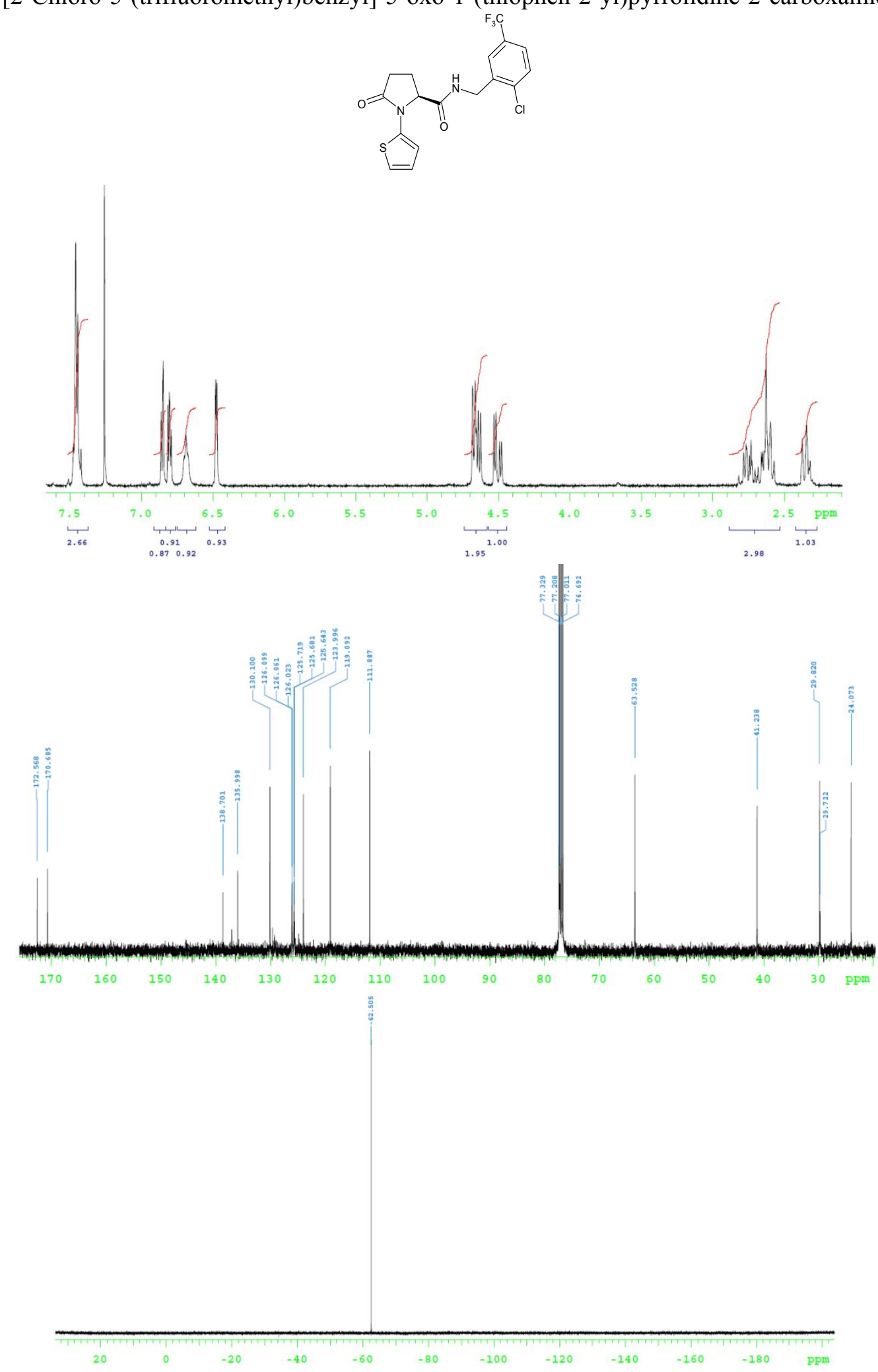
(S)-N-[2-(2,4-Dichlorophenyl)ethyl]-5-oxo-1-phenylpyrrolidine-2-carboxamide (23).

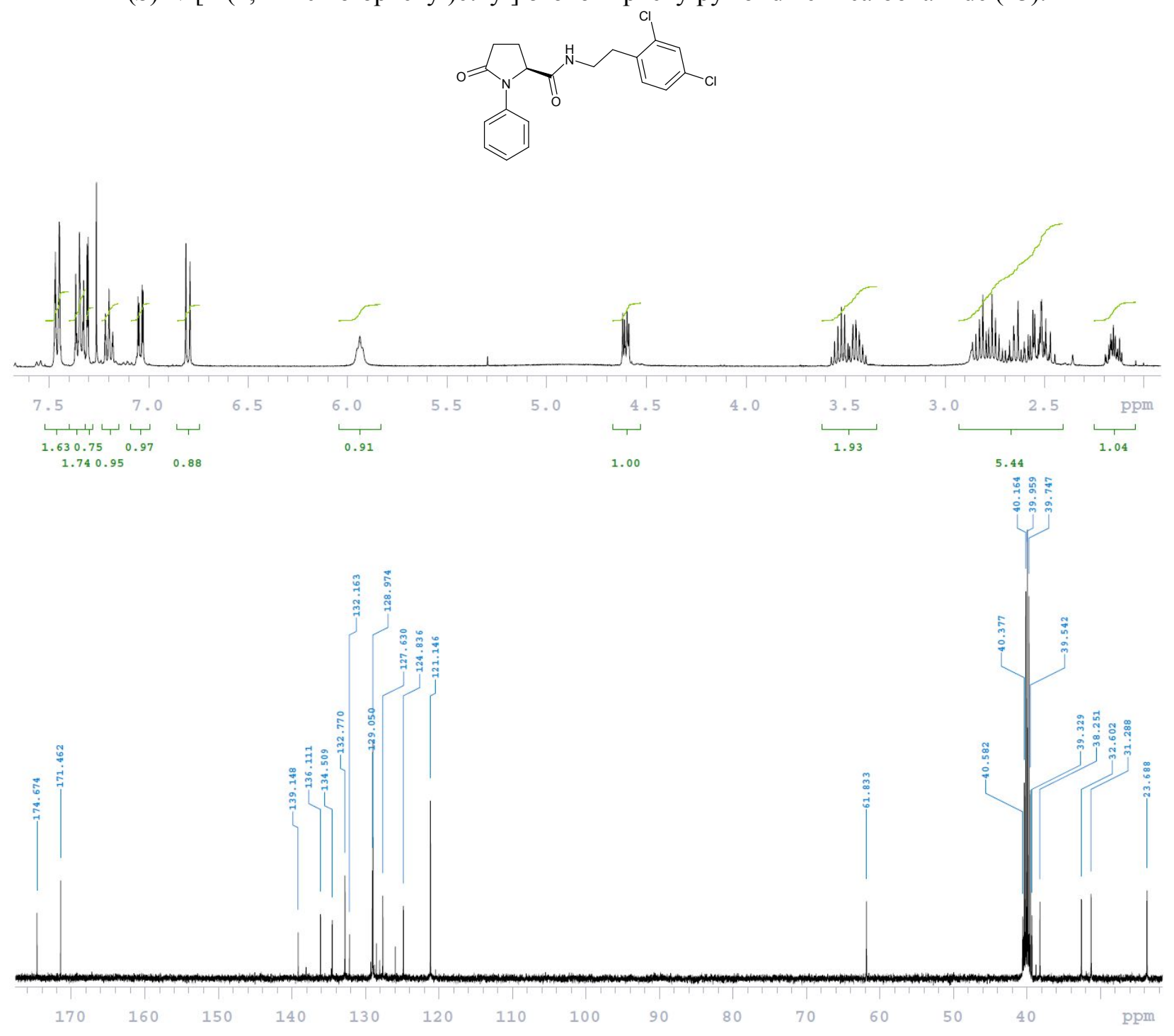


(S)-N,1-bis(2-Chlorobenzyl)-5-oxopyrrolidine-2-carboxamide (30a).

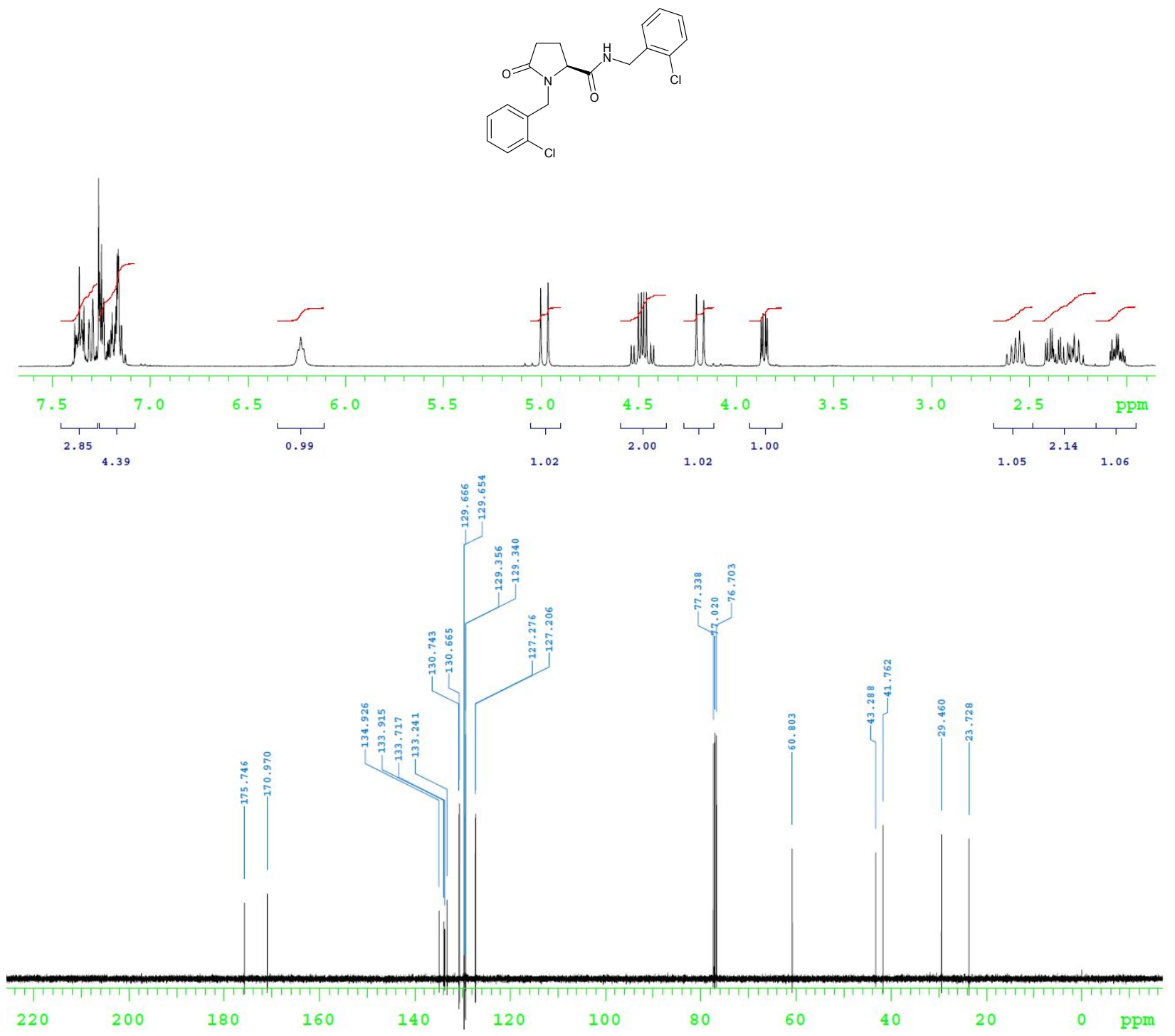


(S)-N-(2-Chlorobenzyl)-1-(4-chlorobenzyl)-5-oxopyrrolidine-2-carboxamide (31a).

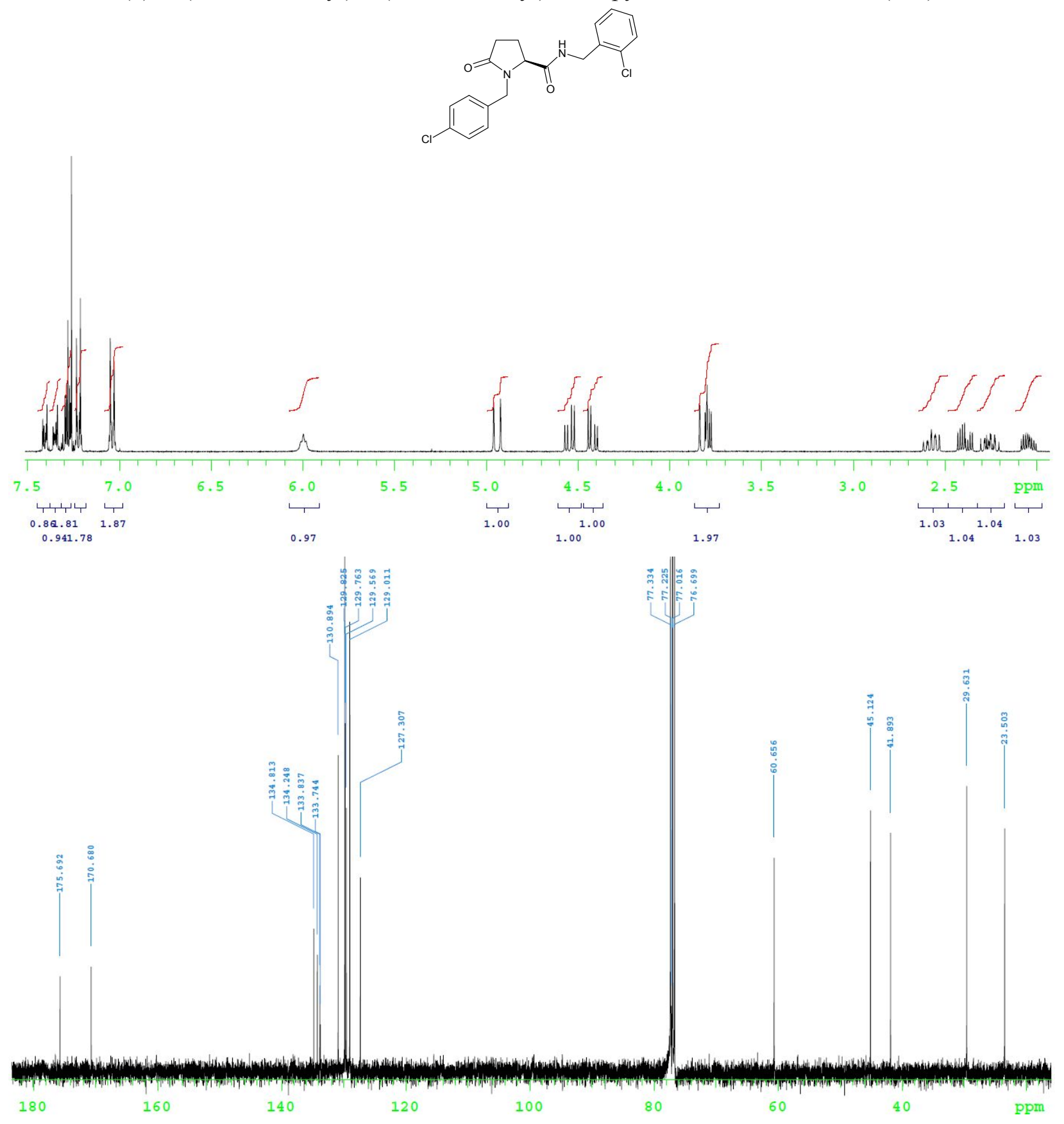


(S)-1-(4-Chlorobenzyl)- $N$-(2,4-dichlorobenzyl)-5-oxopyrrolidine-2-carboxamide (31b).
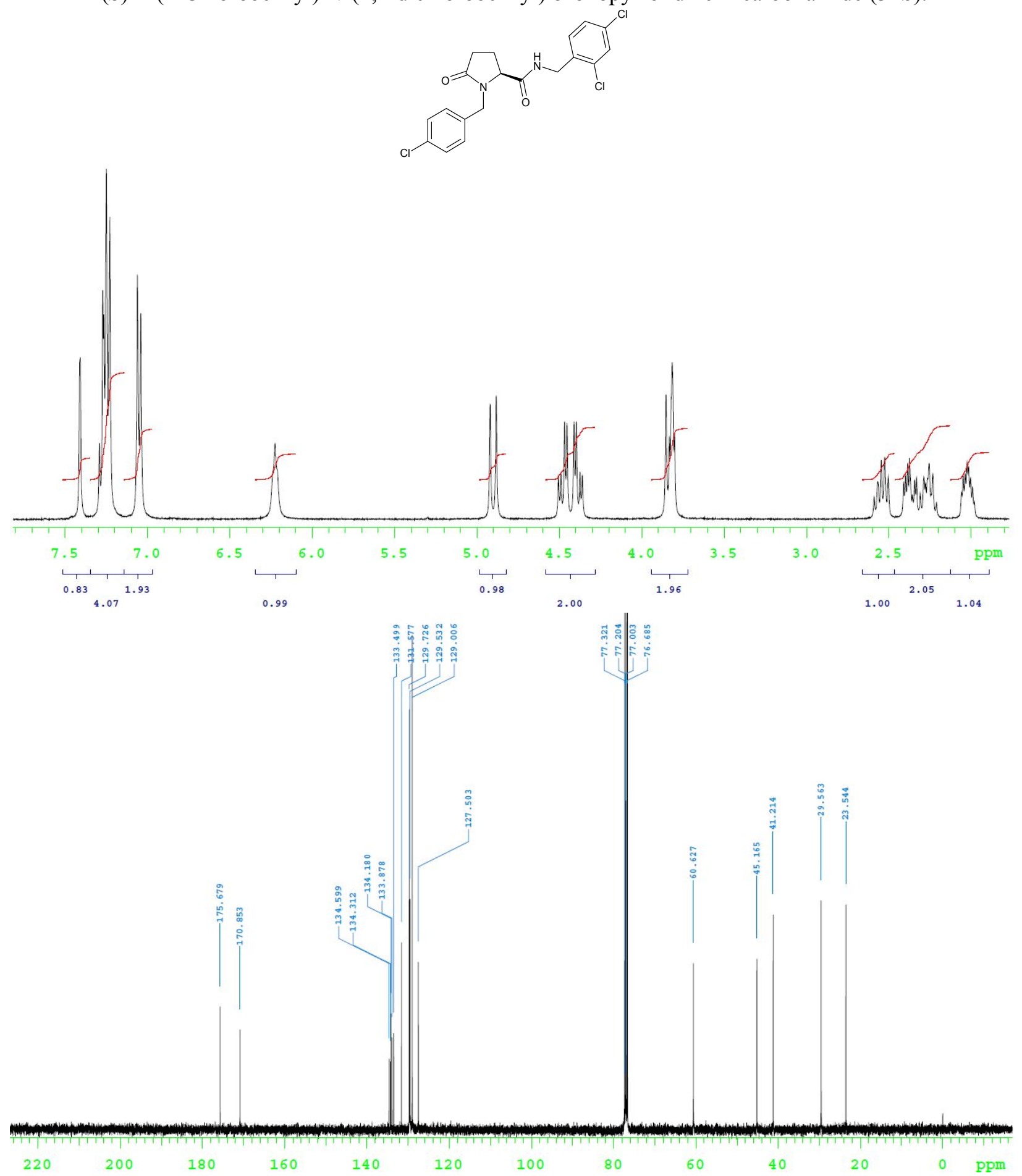
(S)-N-Benzyl-1-(2,4-dichlorobenzyl)-5-oxopyrrolidine-2-carboxamide (32a).

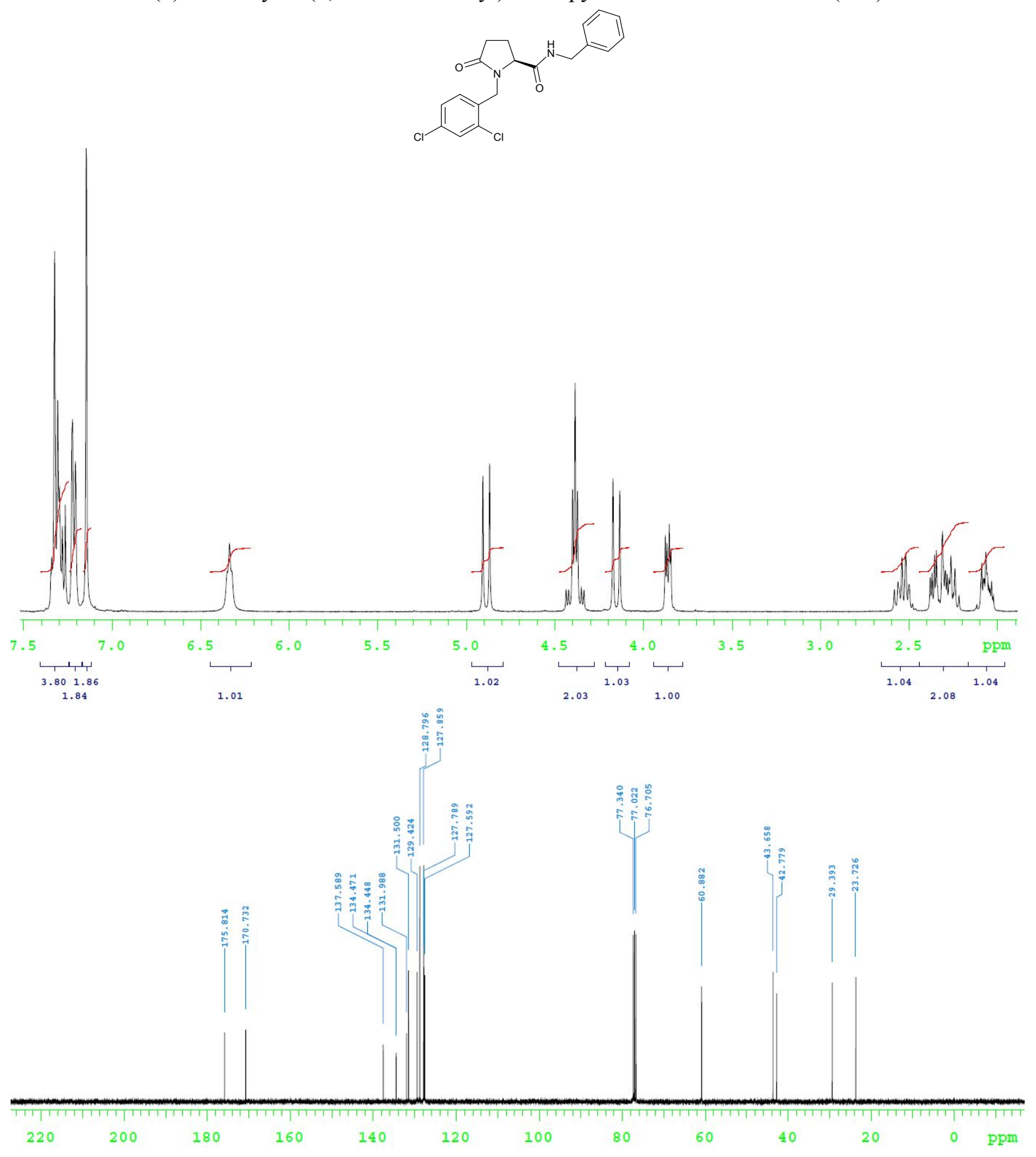


(S)-N-(2-Chlorobenzyl)-1-(2,4-dichlorobenzyl)-5-oxopyrrolidine-2-carboxamide (32b).

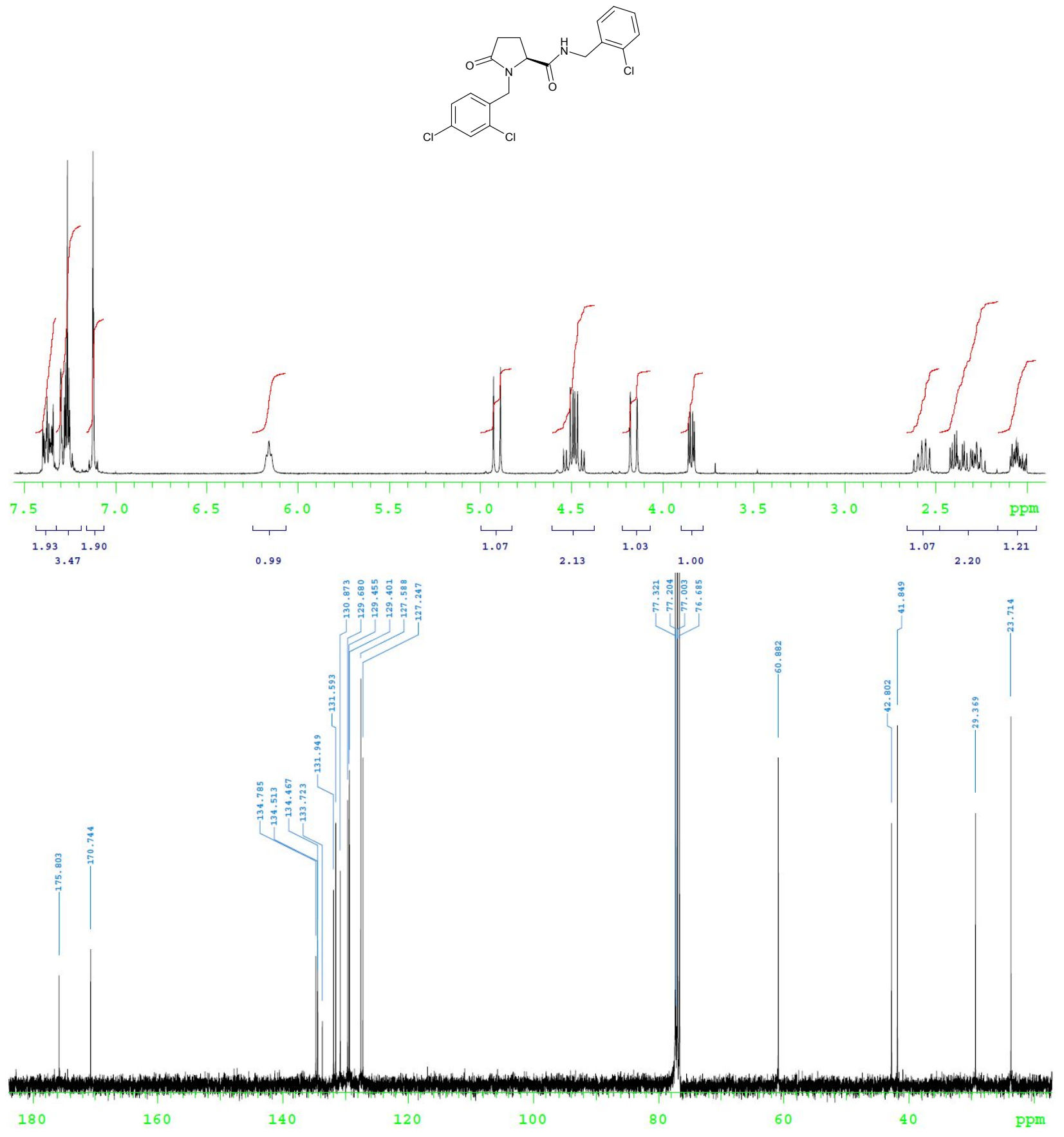


(S)-N,1-bis(2,4-Dichlorobenzyl)-5-oxopyrrolidine-2-carboxamide (32c).

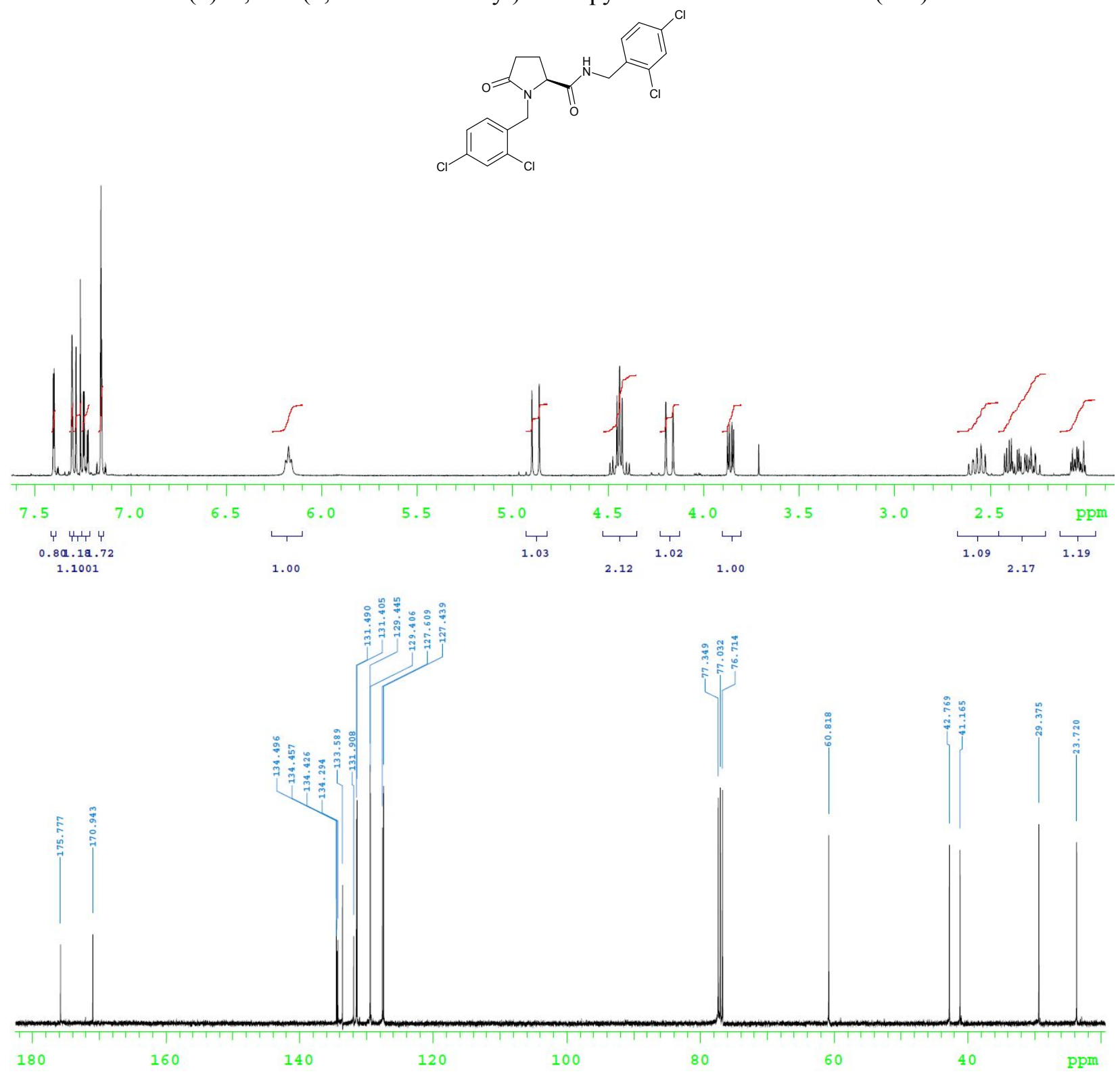


(S)-1-(2,4-Dichlorobenzyl)- $N$-(2,4-dimethoxybenzyl)-5-oxopyrrolidine-2-carboxamide (32d).
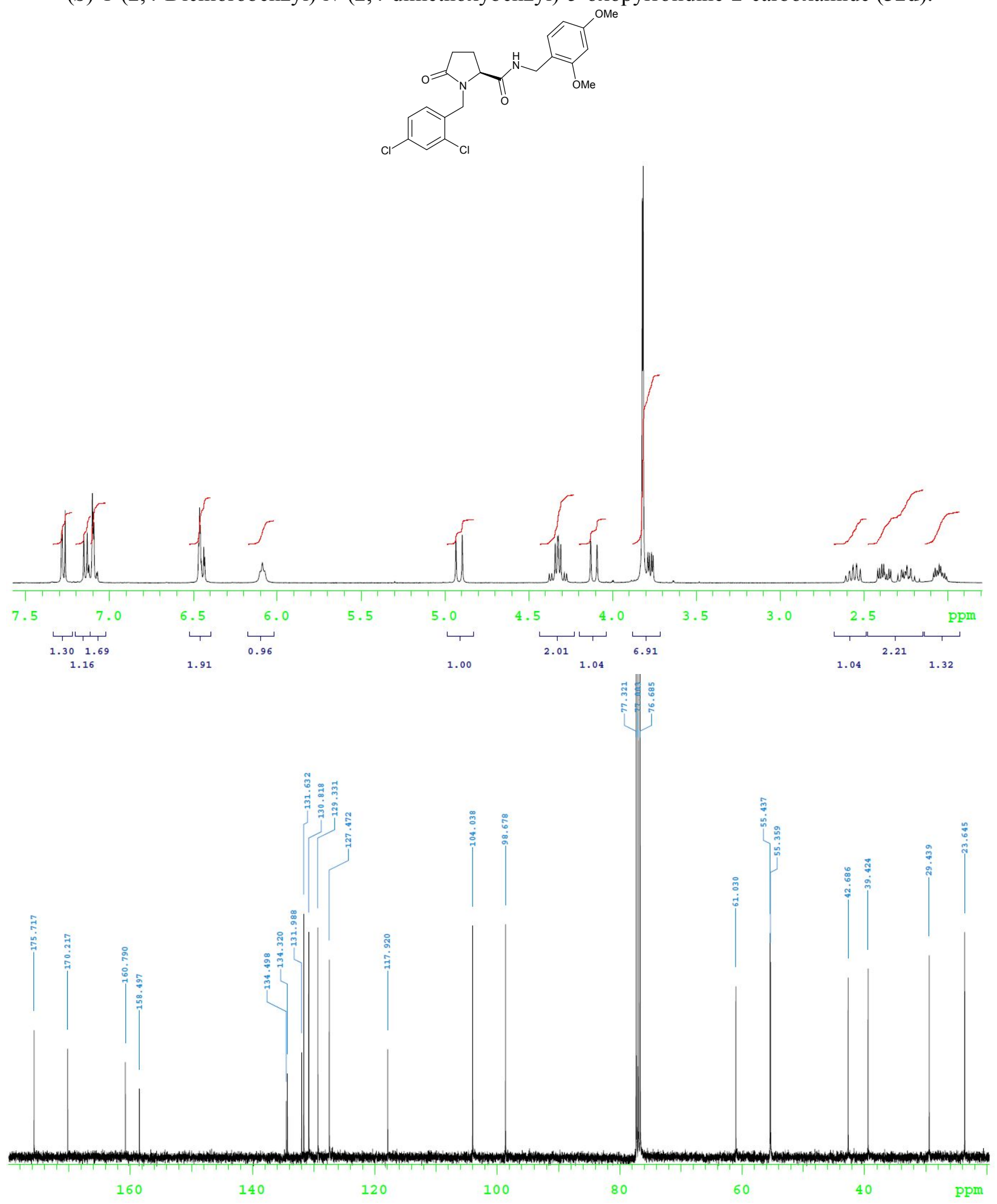
(S)-1-(2,4-Dichlorobenzyl)- $N$-(furan-2-ylmethyl)-5-oxopyrrolidine-2-carboxamide (32e).

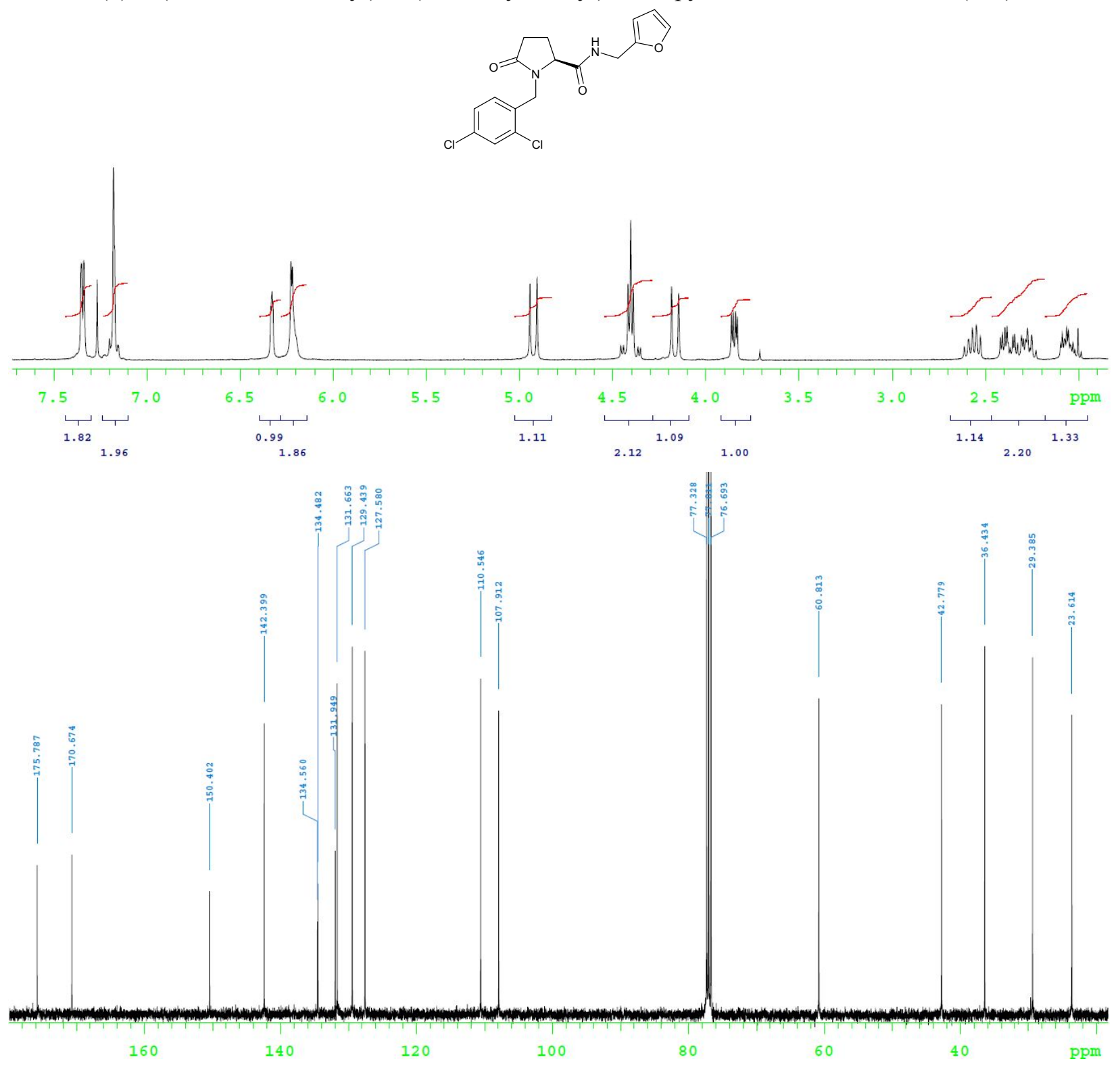


(S)- $N$-Benzyl-1-(2,4-dichlorobenzyl)- $N$-methyl-5-oxopyrrolidine-2-carboxamide (34).

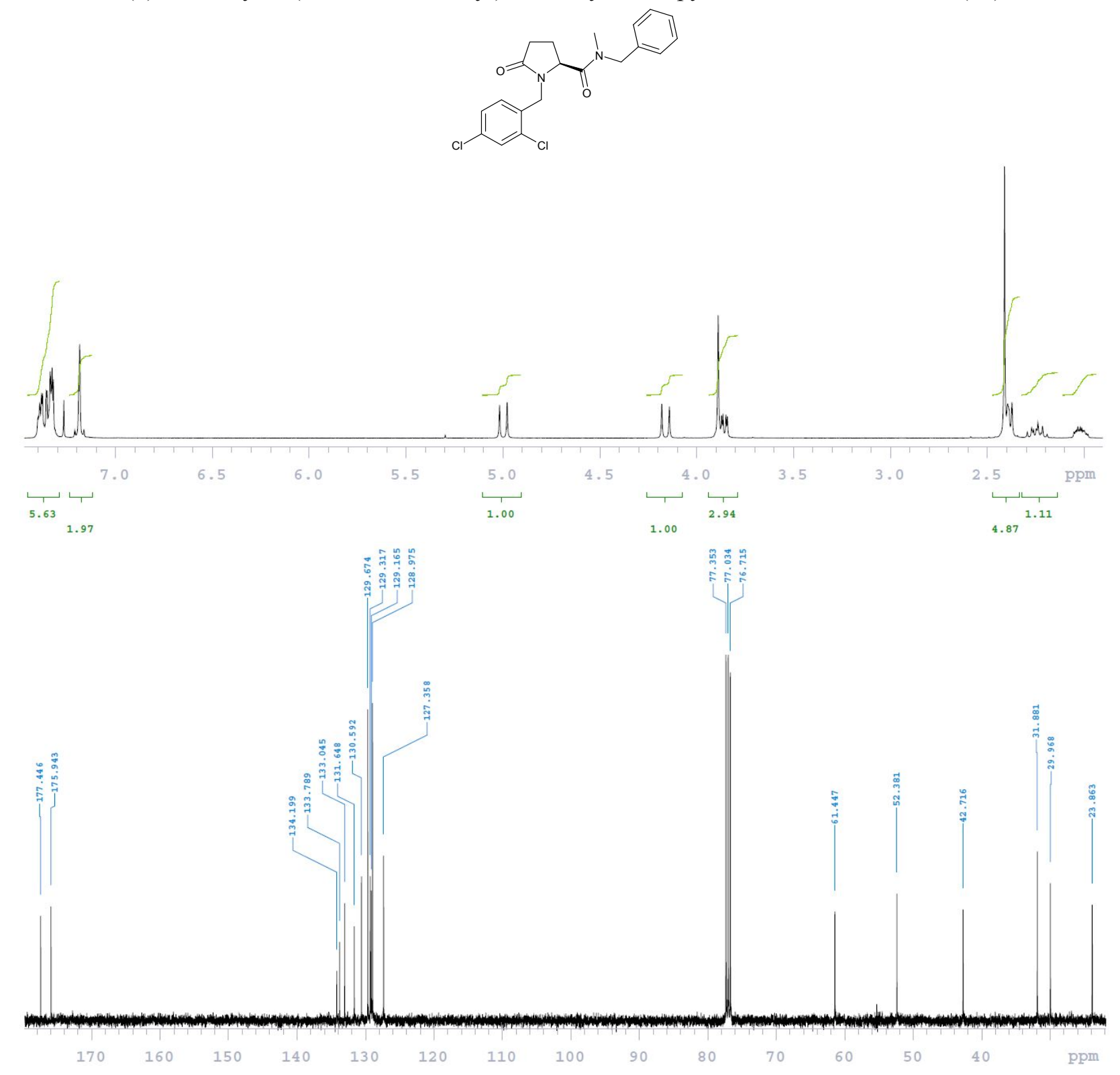


(S)-1-Benzyl-5-oxo- $N$-(2-phenylethyl)pyrrolidine-2-carboxamide (37).

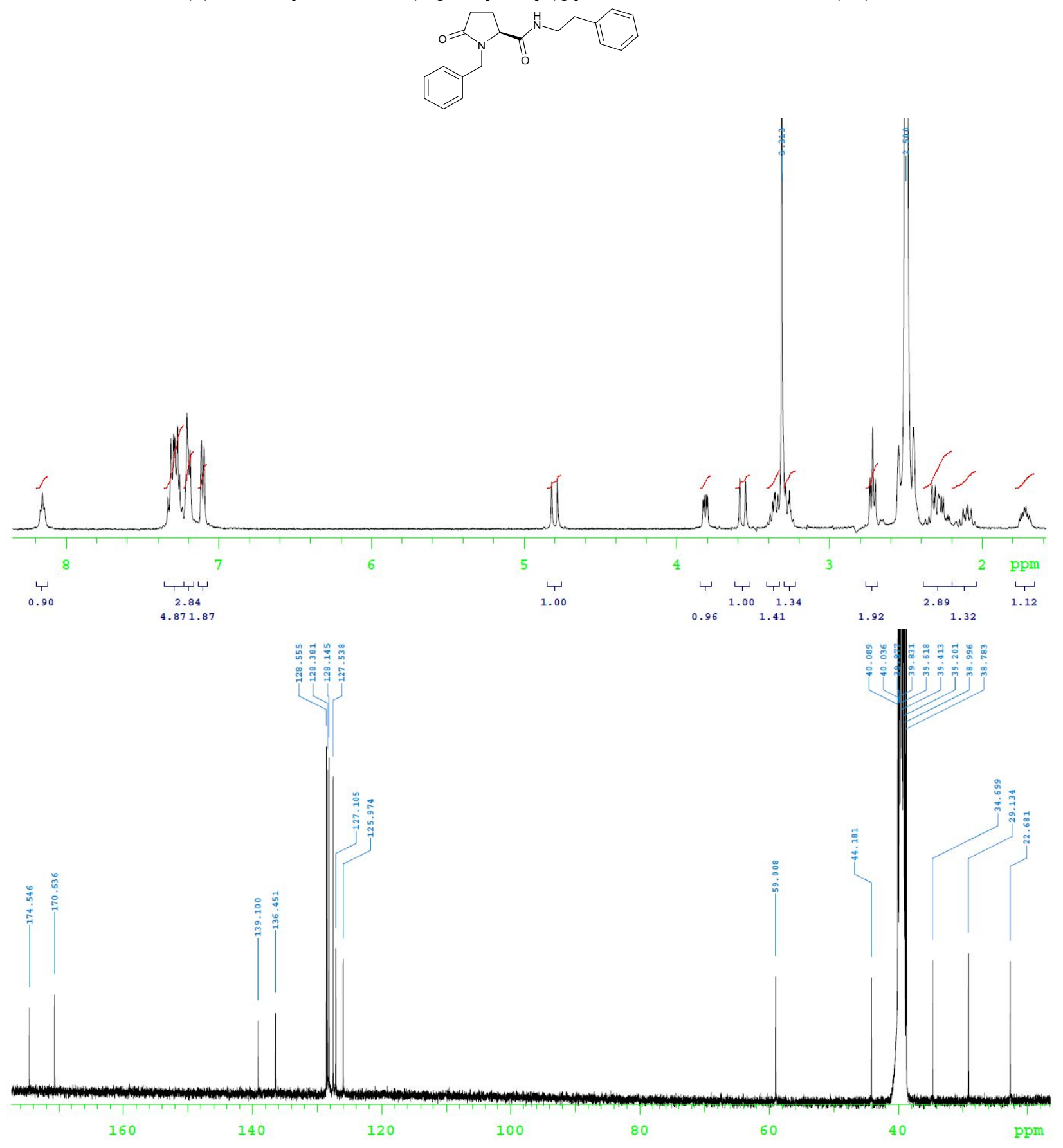


(S)-1-(4-Chlorobenzyl)-5-oxo- $N$-(2-phenylethyl)pyrrolidine-2-carboxamide (38).

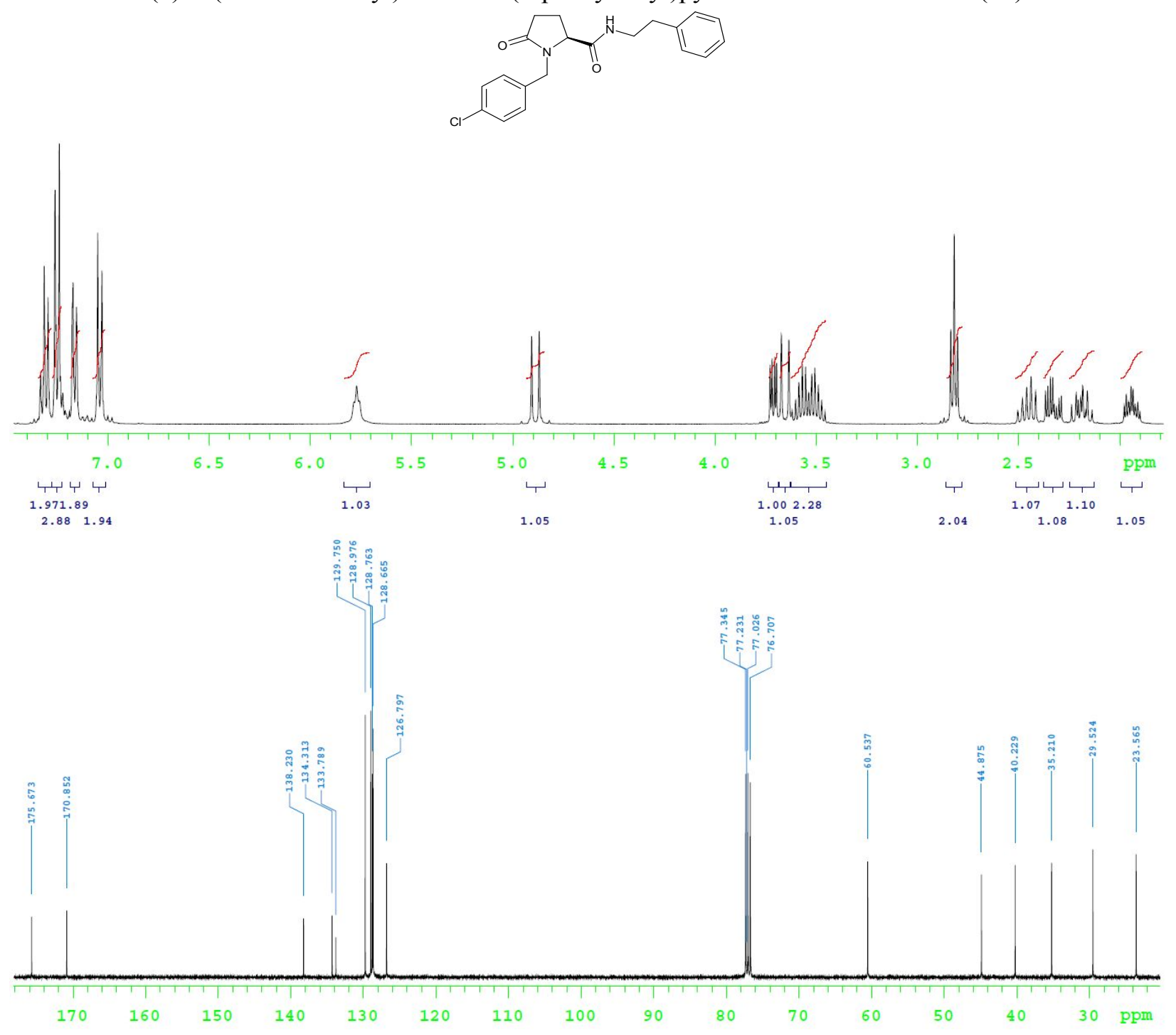


(S)-1-(4-Bromobenzyl)-5-oxo-N-(2-phenylethyl)pyrrolidine-2-carboxamide (39).

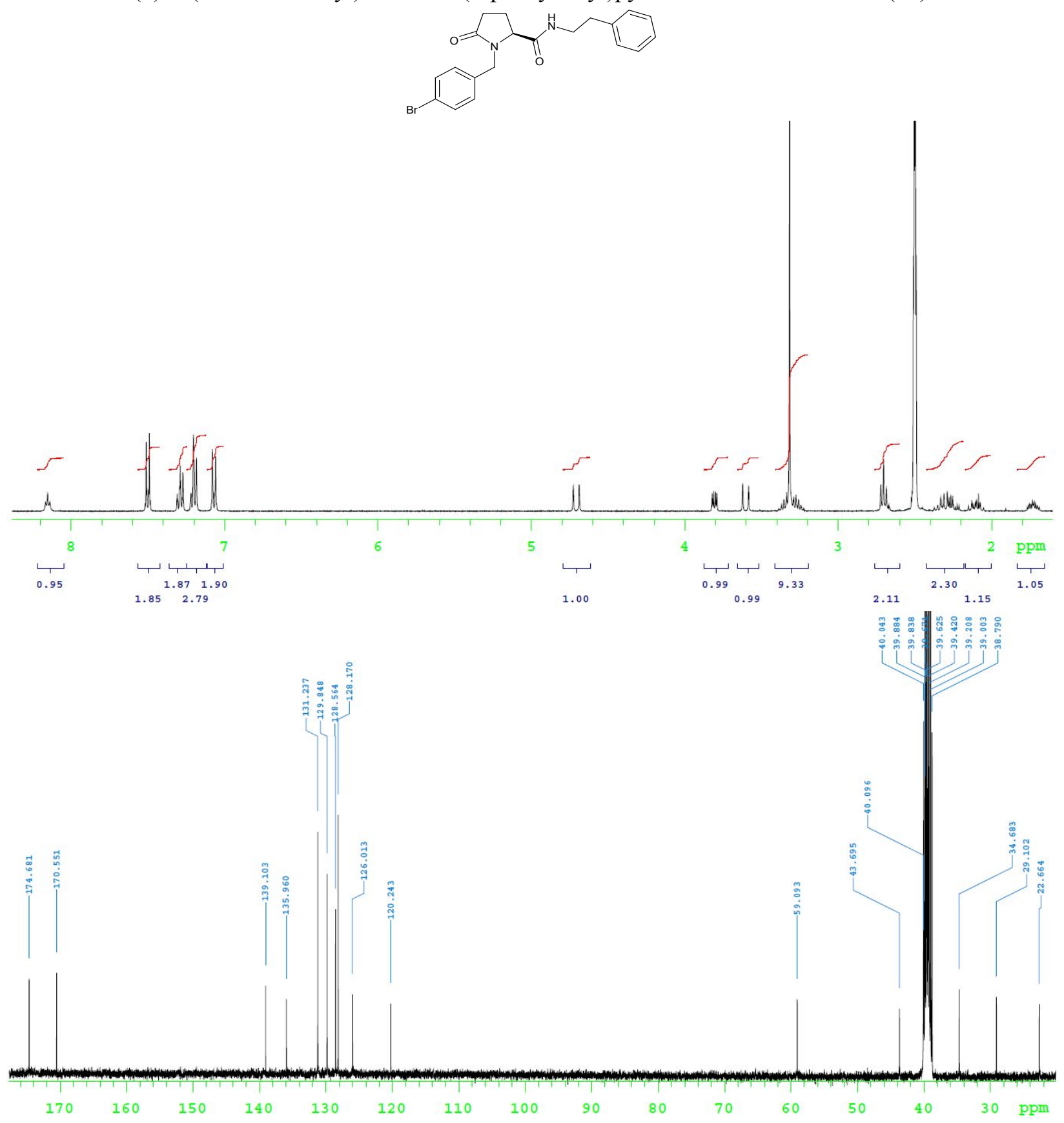


(S)-1-(4-Methylbenzyl)-5-oxo- $N$-(2-phenylethyl)pyrrolidine-2-carboxamide (40).

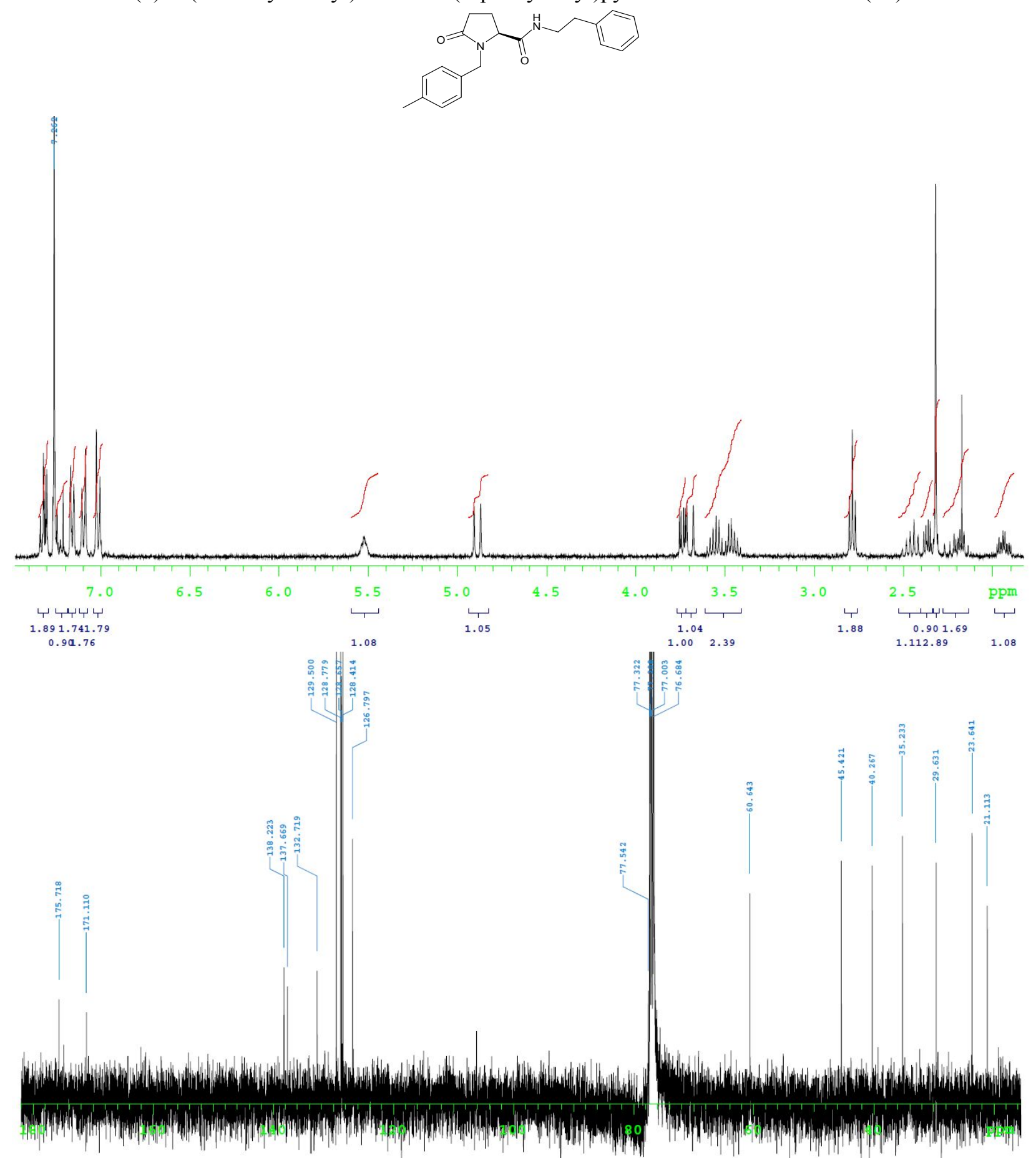


(S)-1-(4-Methoxybenzyl)-5-oxo- $N$-(2-phenylethyl)pyrrolidine-2-carboxamide (41).

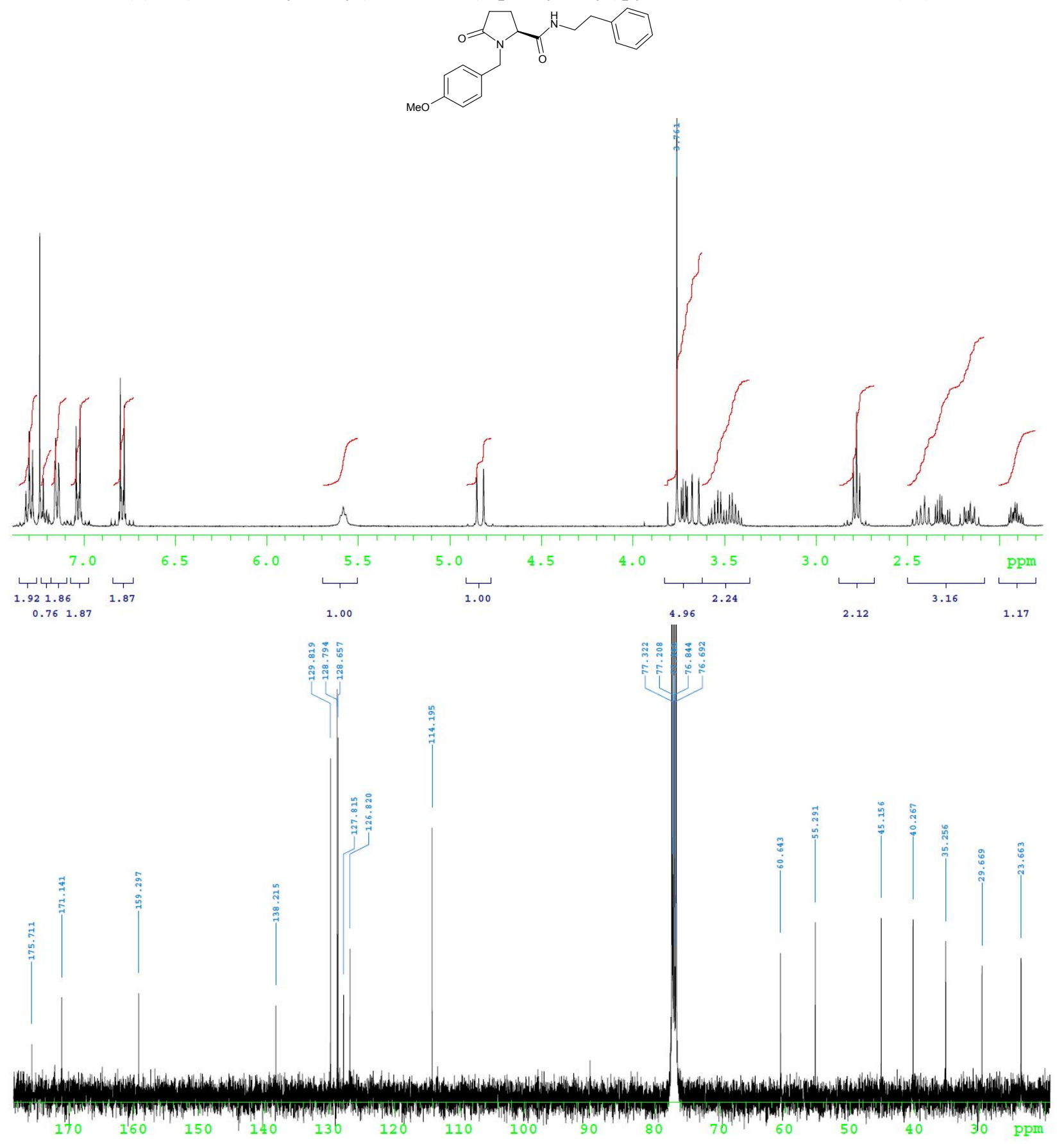


(S)-1-(2,4-dichlorobenzyl)-5-oxo- $N$-(2-phenylethyl)pyrrolidine-2-carboxamide (42).

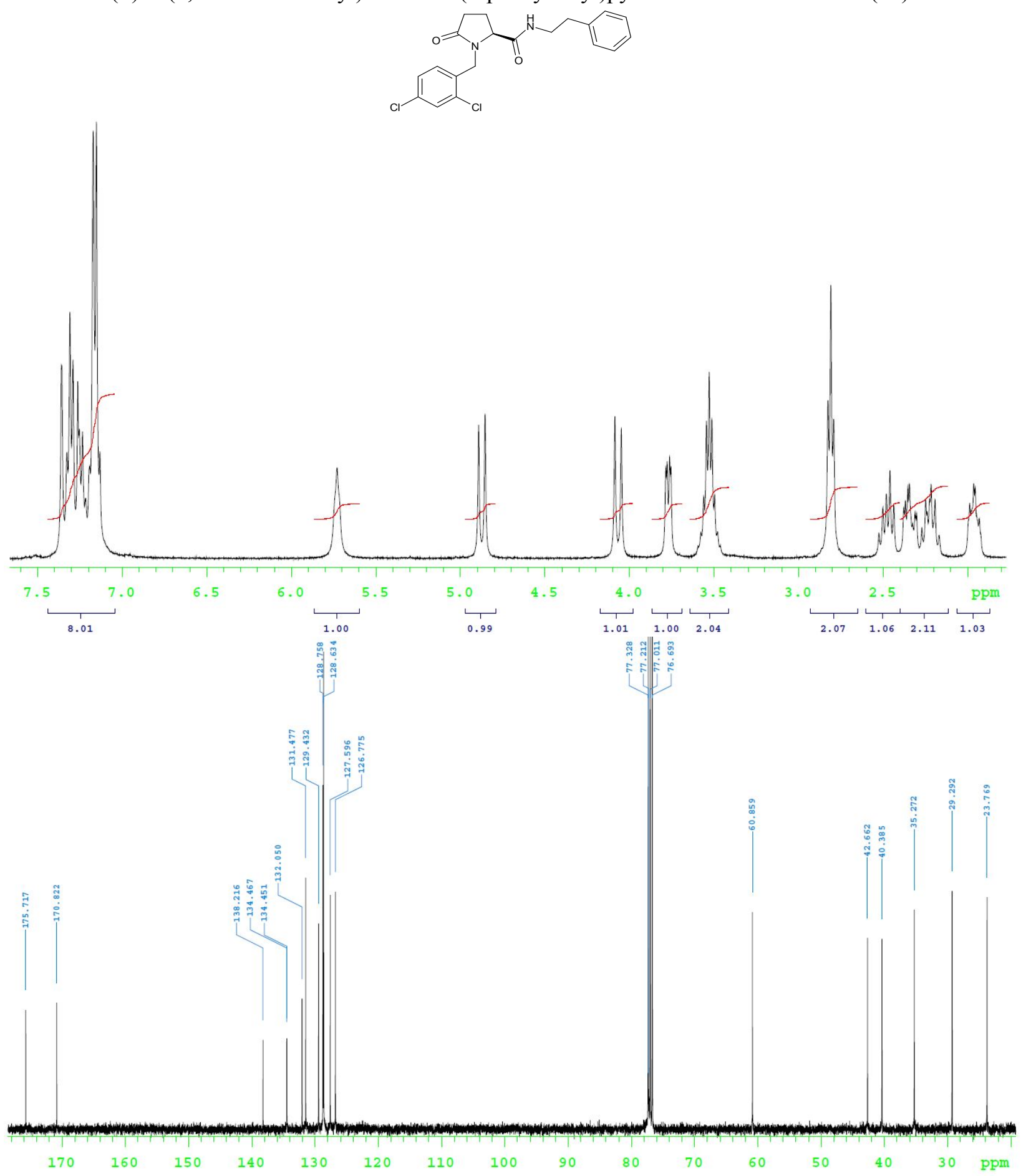


(S)-1-(4-nitrobenzyl)-5-oxo- $N$-(2-phenylethyl)pyrrolidine-2-carboxamide (43).

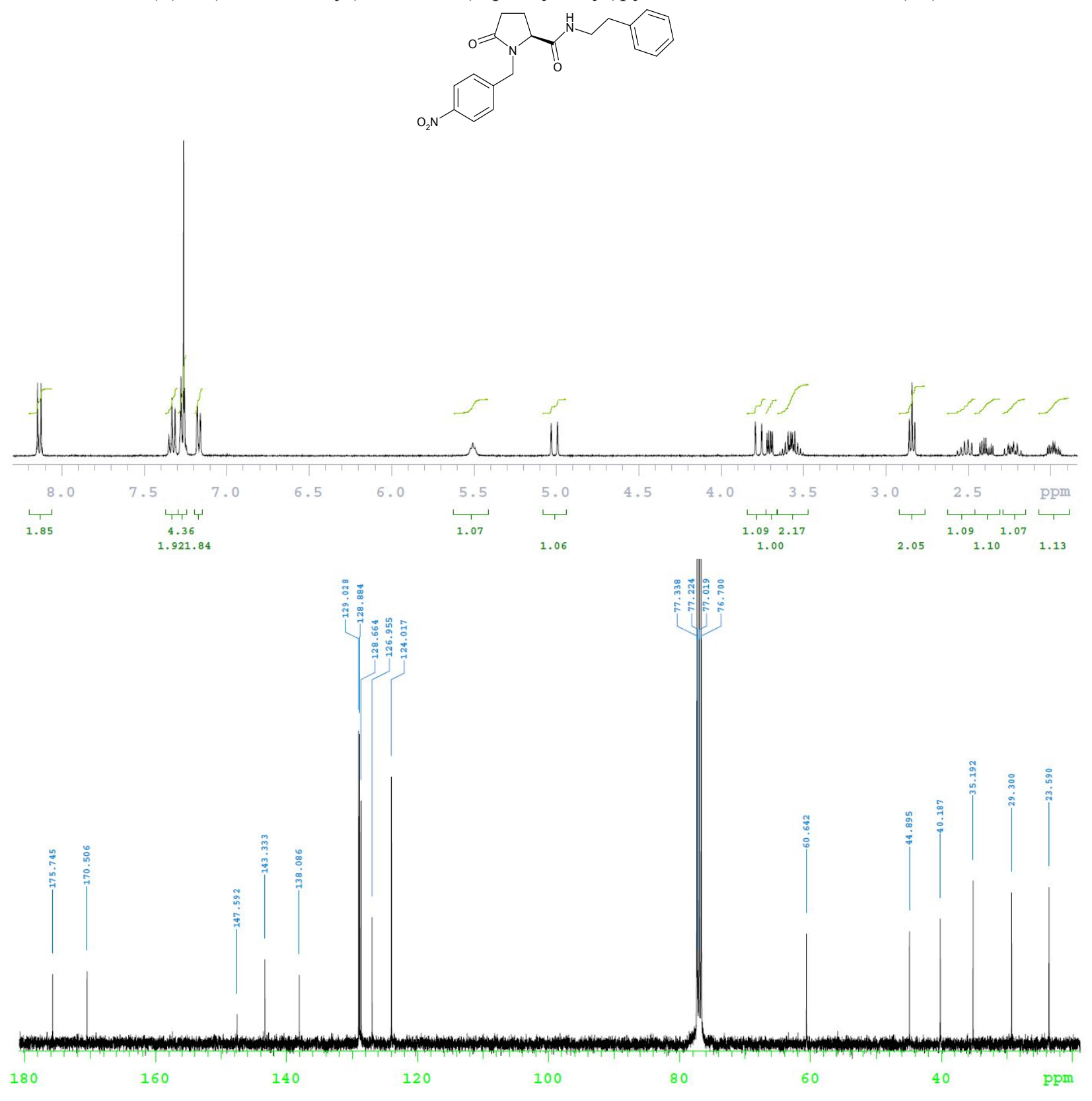


(S)-N-(2,4-Dichlorobenzyl)-4-hydroxyimino-5-oxo-1-(thiophen-2-yl)pyrrolidine-2-carboxamide (45).

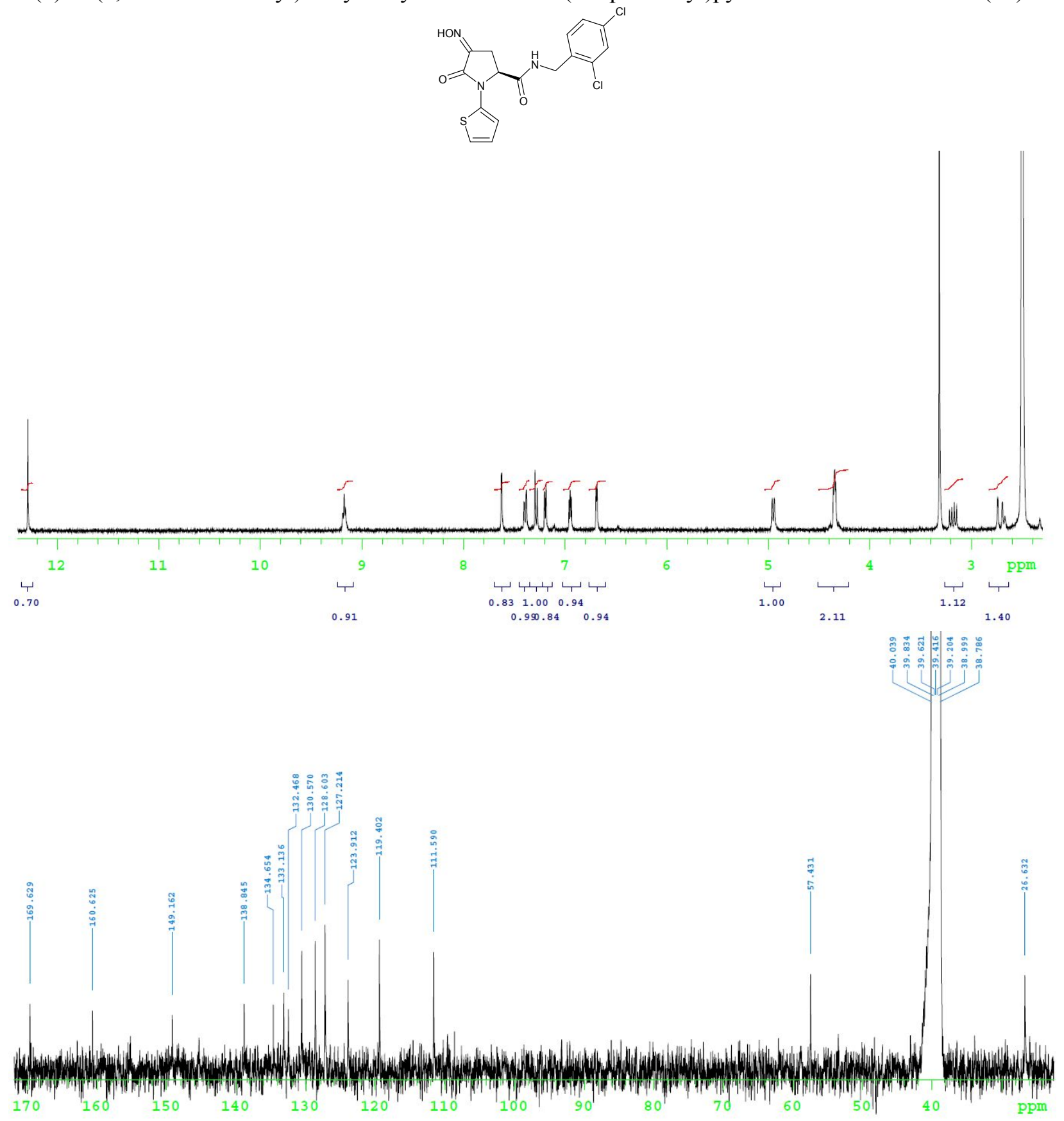


(S)-Methyl 4-[(dichlorobenzylamino)methylidene]-5-oxopyrrolidine-1,2-dicarboxylate (47).

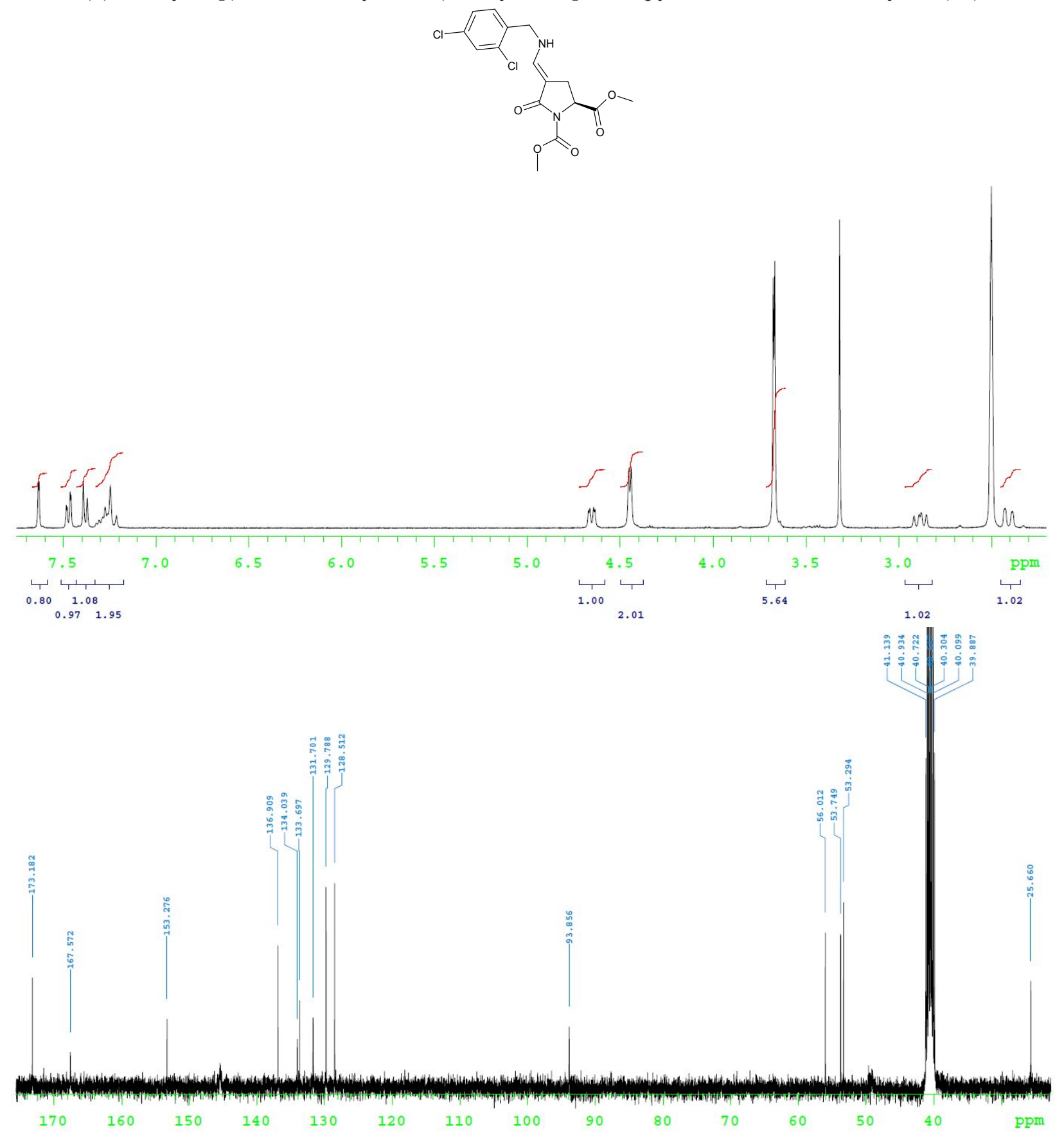


(S)-N-(2,4-Dichlorobenzyl)-2-oxo-3-(thiophen-2-yl)-1,3-oxazolidine-4-carboxamide (60).<smiles>O=C(NCc1ccc(Cl)cc1Cl)C1COC(=O)N1c1cccs1</smiles>

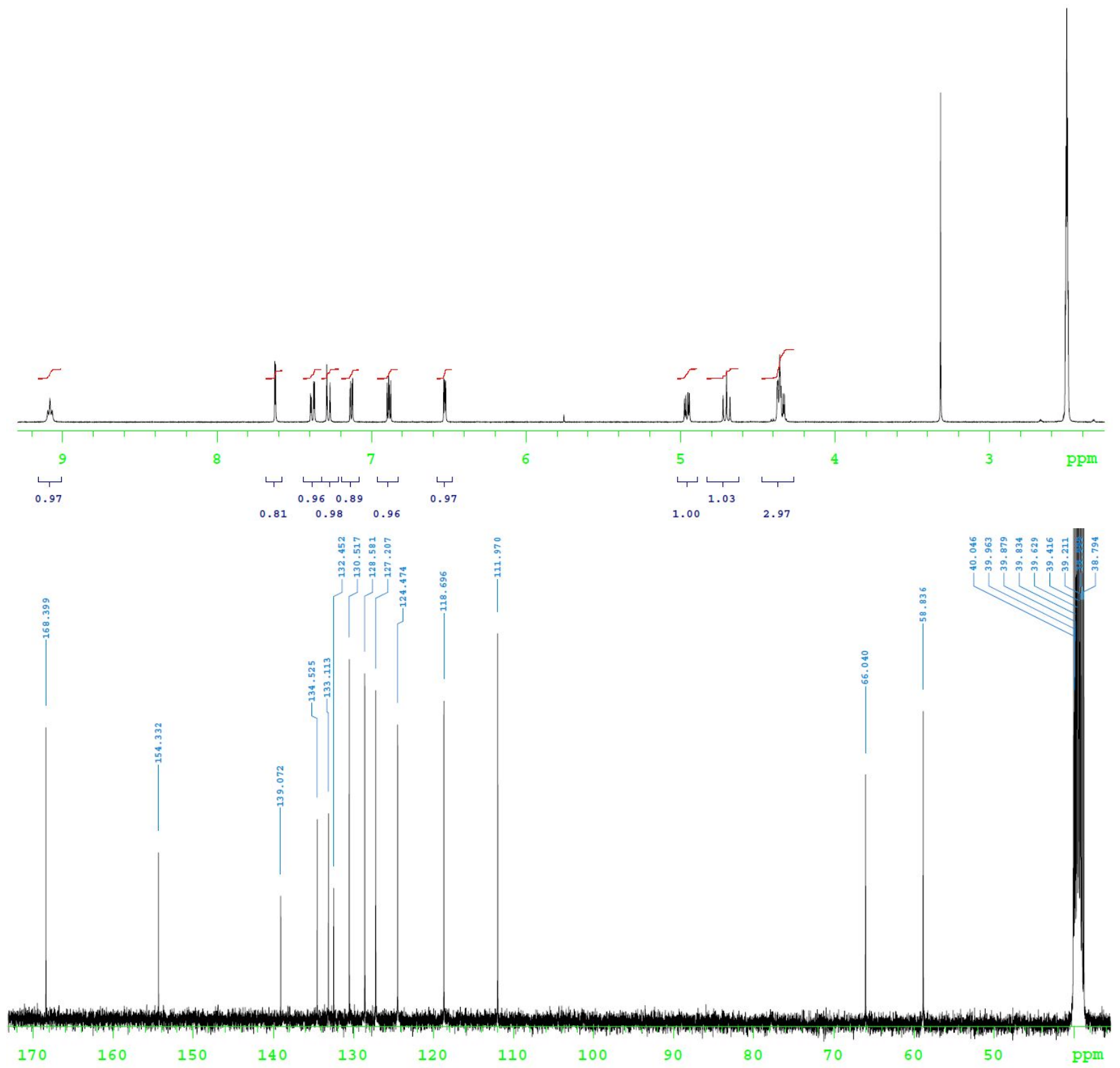


$N$-(2,4-Dichlorobenzyl)-5-oxopyrrolidine-3-carboxamide (64).<smiles>O=C1CC(C(=O)NCc2ccc(Cl)cc2Cl)CN1</smiles>
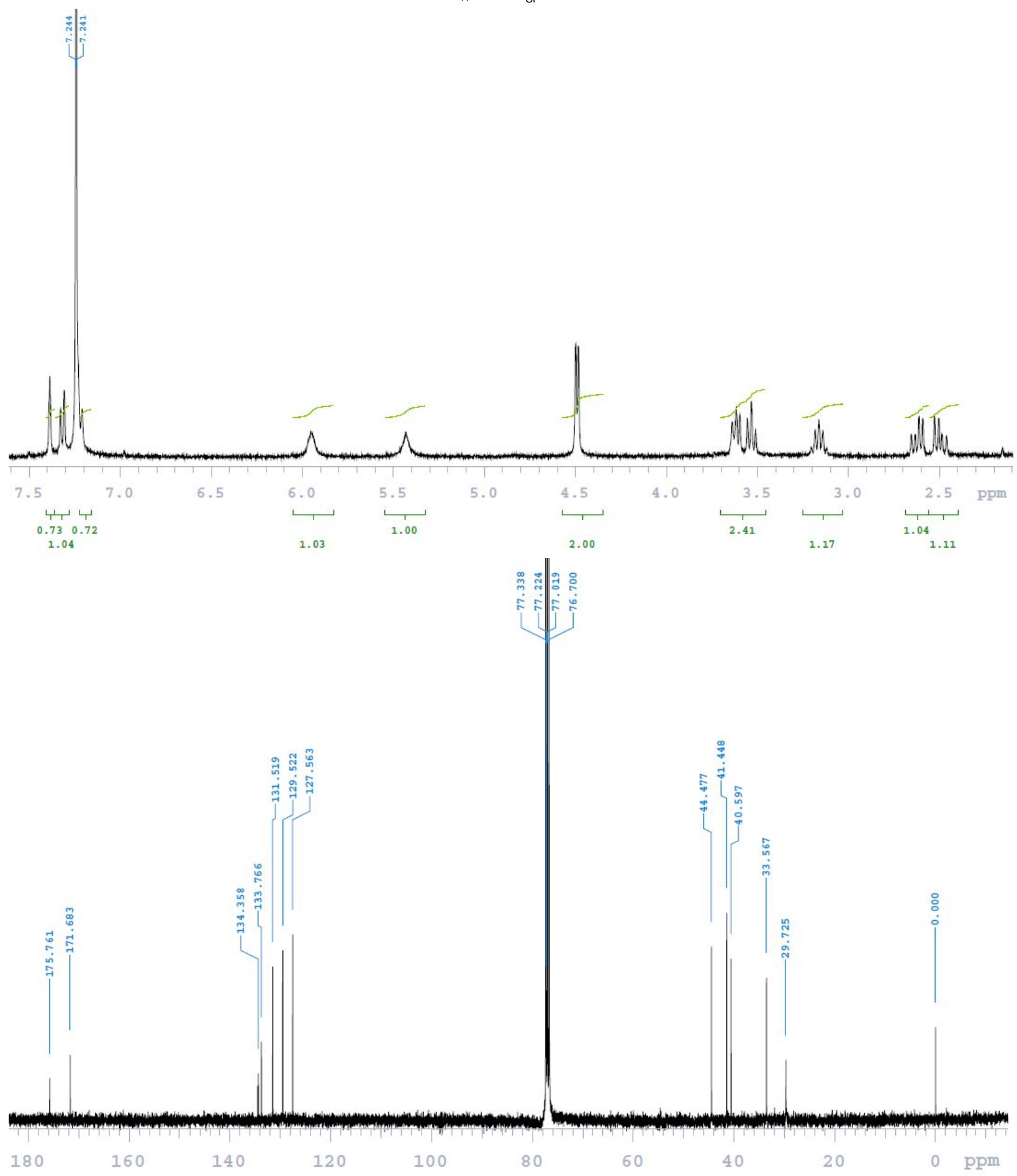
$N$-(2,4-Dichlorobenzyl)-5-oxo-1-thien-2-ylpyrrolidine-3-carboxamide (65).

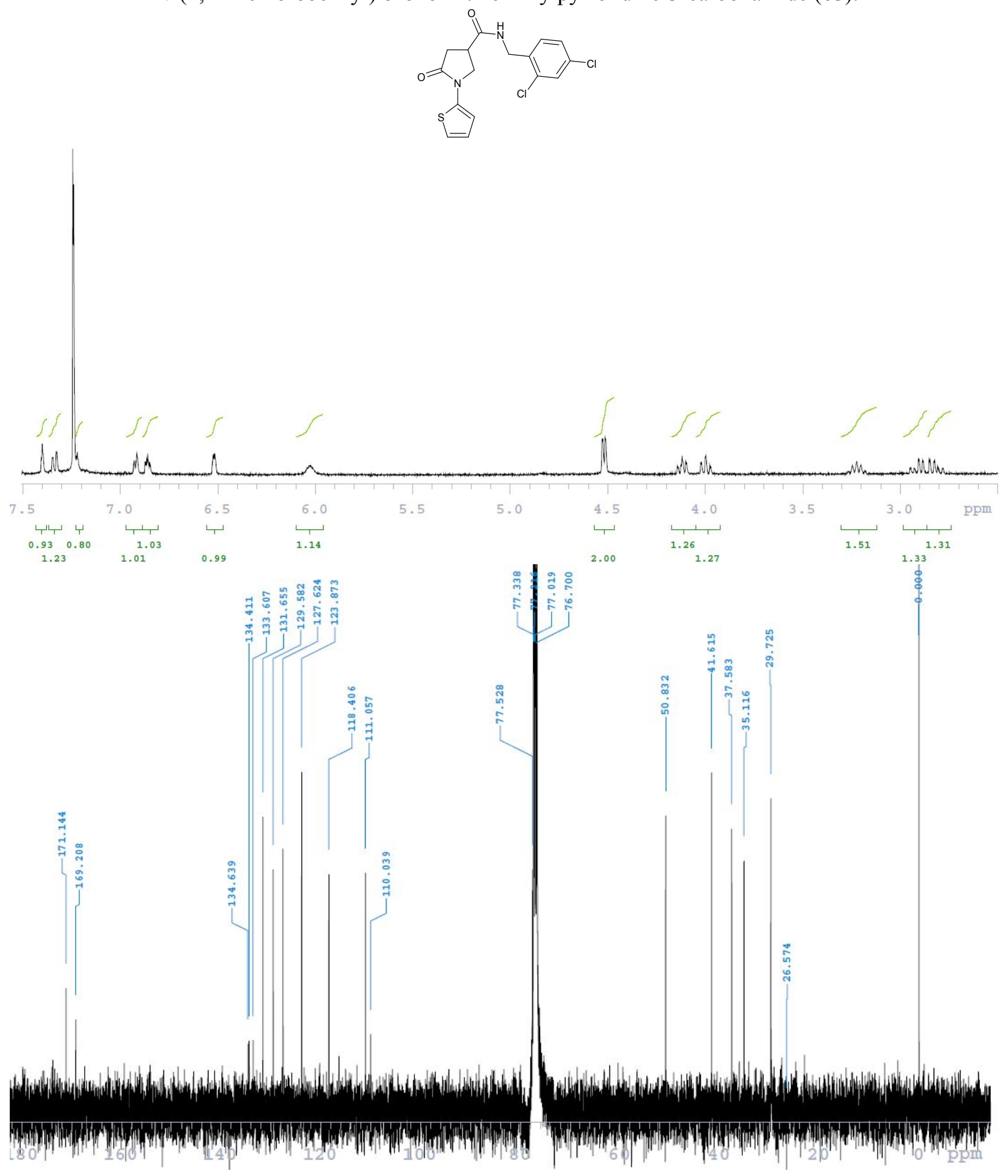


$N$-(2,4-Dichlorobenzyl)pyridine-2-carboxamide (67).<smiles>O=C(NCc1ccc(Cl)cc1Cl)c1ccccn1</smiles>
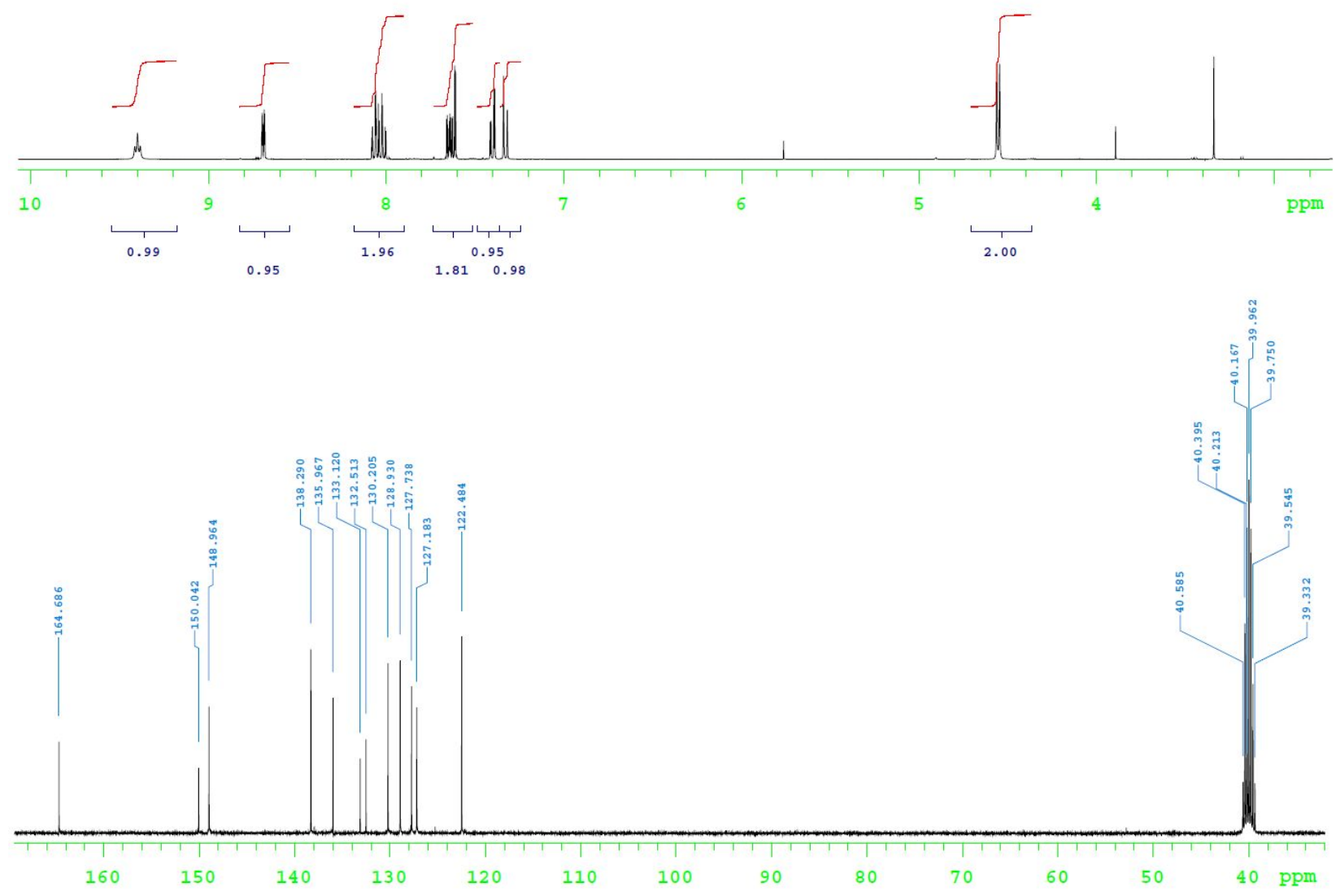

$40 \mathrm{ppm}$ 
2-\{[(2,4-Dichlorobenzyl)amino]carbonyl $\}$-1-methylpyridinium iodide (68).<smiles></smiles>
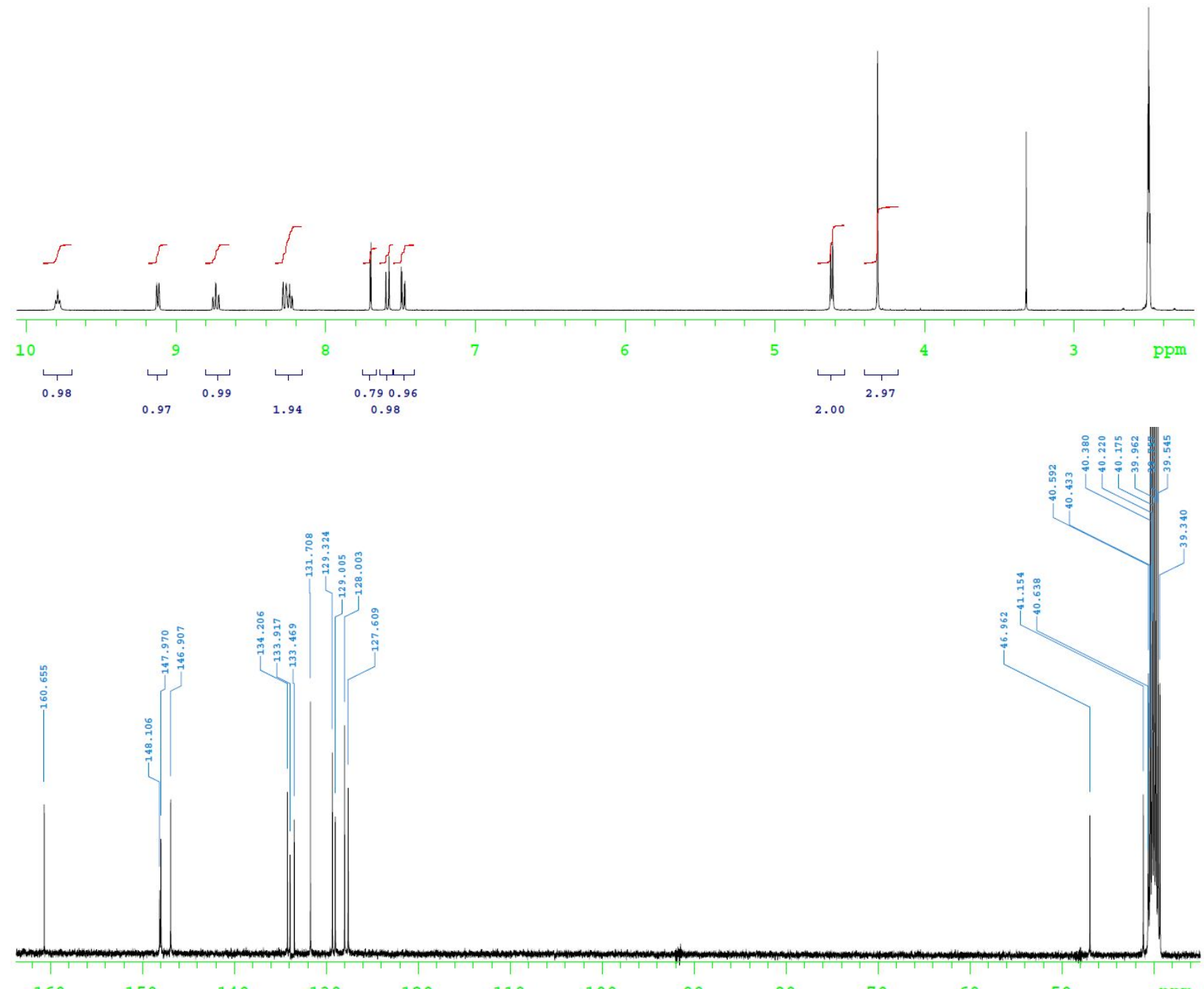

160

150

140

130

120

110

100

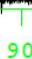

80

70

60

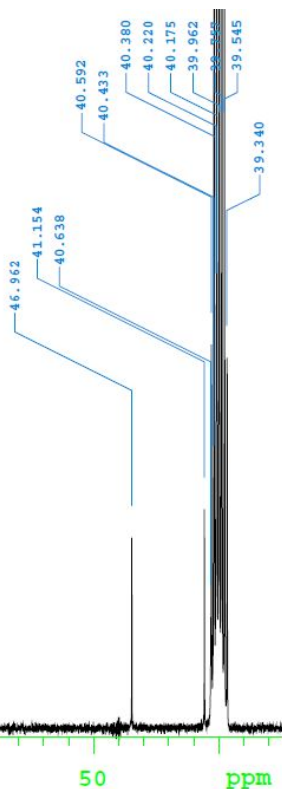


(S,E)-2-Cyano-1-(1-phenylethyl)-3-(quinolin-5-yl)guanidine (74a).

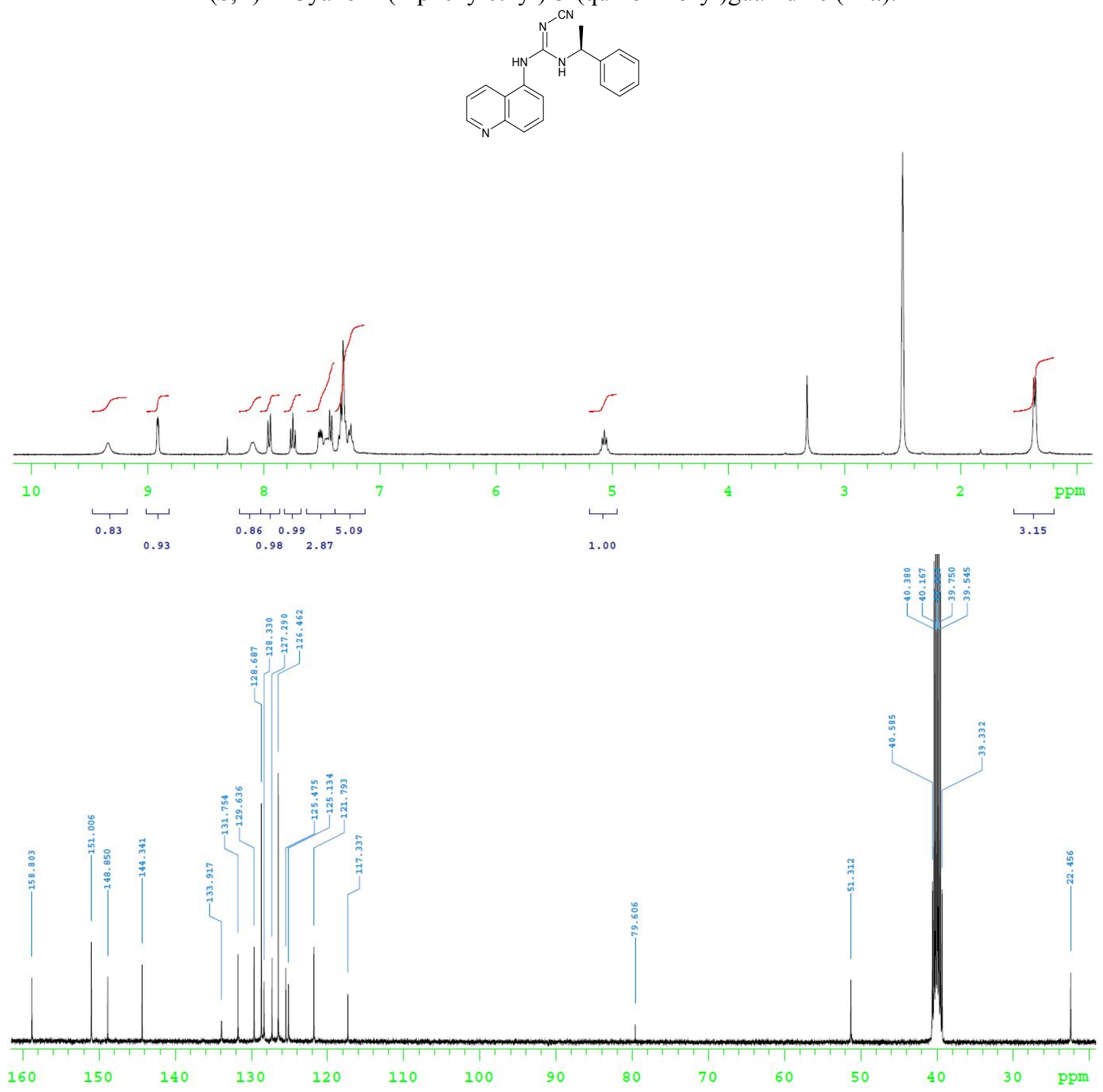




\section{Chiral separation}

Materials and methods

Stationary phases:

The chiral analytical column used for this study, was a Chiralpak AD-H, purchased from Chiral Technologies Europe (Illkirch, France). This column had dimensions $250 \mathrm{~mm}$ x $4.6 \mathrm{~mm}$ i.d. with $5 \mu \mathrm{m}$ particle size and was coated on a silica-gel support.

\section{Chromatographic system and conditions:}

The chromatographic system used was an SFC-PICLAB hybrid 10-20 apparatus (PIC Solution, Avignon, France) equipped with an autosampler comprised a 48-vial plate and a 24-vial plate (model Alias, Emmen, Netherlands), three model 40P pumps: two for $\mathrm{CO}_{2}$ and a third for the modifier (Knauer, Berlin, Germany), a column oven with a Valco ten-position column selection valve, and a Valco six-position solvent switching valve. The proportion of the modifier in the mobile phase was adjusted by a piston pump. It was then directly added in the $\mathrm{CO}_{2}$ feeding, and the mixture of the both (modifier and $\mathrm{CO}_{2}$ ) was pumped by another piston pump at the total flow rate. The pump head used for pumping the $\mathrm{CO}_{2}$ was cooled to $-8^{\circ} \mathrm{C}$ by a cryostat (model Minichiller, Huber, Offenburg, Germany). The injection valve was supplied with $19.6 \mu \mathrm{L}$ sample loops. The system was also composed of a Smartline 2600 diode array detector (DAD) (Knauer, Berlin, Germany). Detection wavelength was set at $210 \mathrm{~nm}$. After the detector, the outlet pressure was controlled by a back-pressure regulator (BPR). The outlet regulator tube was heated to $55^{\circ} \mathrm{C}$ to avoid ice formation during the $\mathrm{CO}_{2}$ depressurization. The system was controlled and the data were acquired with the SFC PicLab Analytic Online v.3.1.2 software and the data were processed with the Analytic Offline v.3.2.0 software (PIC Solution, Avignon, France). During the separation optimization, the mobile phase was $\mathrm{CO}_{2}$-modifier mixtures with the proportion of methanol of $20 \%$, flow rate was $4 \mathrm{~mL} / \mathrm{min}$. All analyses were run in isocratic mode. The column oven temperature was $40^{\circ} \mathrm{C}$ and the outlet pressure was maintained at 120 bar for all experiments.

\section{$\underline{\text { Results }}$}

Chiral separation of racemate 16i resulted in resulted in two isomers (Figure S15-1).

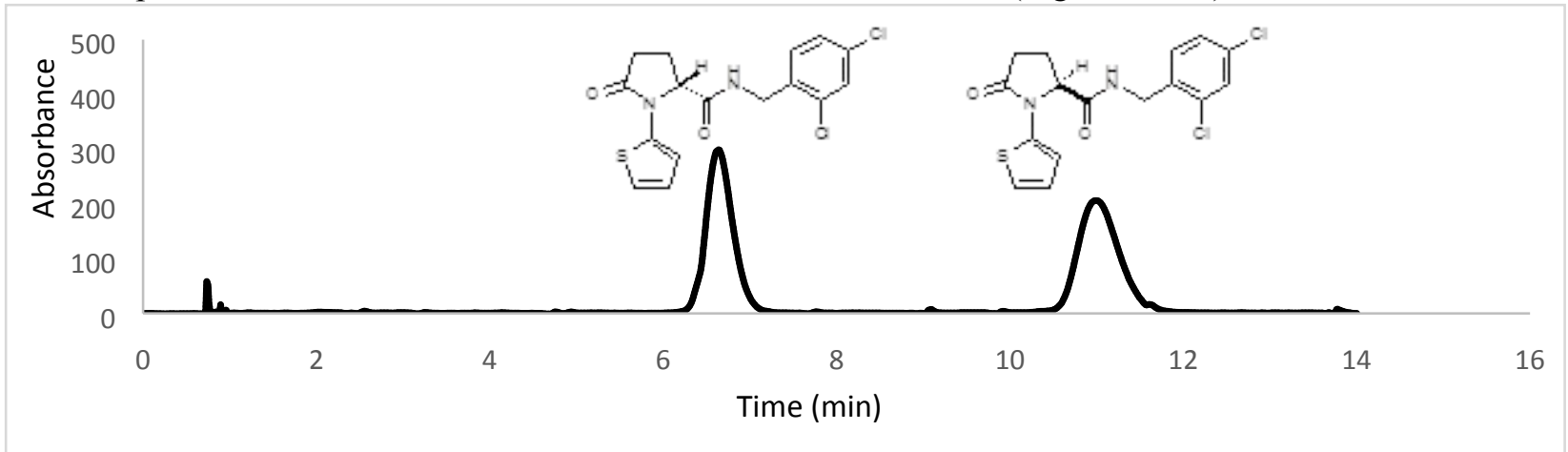

Figure S3-1. Supercritical Fluid Chromatography of Racemate of Compound 16i resulted in two isomers $\left(\mathrm{t}_{\mathrm{R} 1}, 6.6 \mathrm{~min}\right.$; $\left.\mathrm{t}_{\mathrm{R} 2}, 11.4 \mathrm{~min}\right)$. 
Chiral separation of enantiopure compound 16i resulted in one isomer (Figure S1-2).

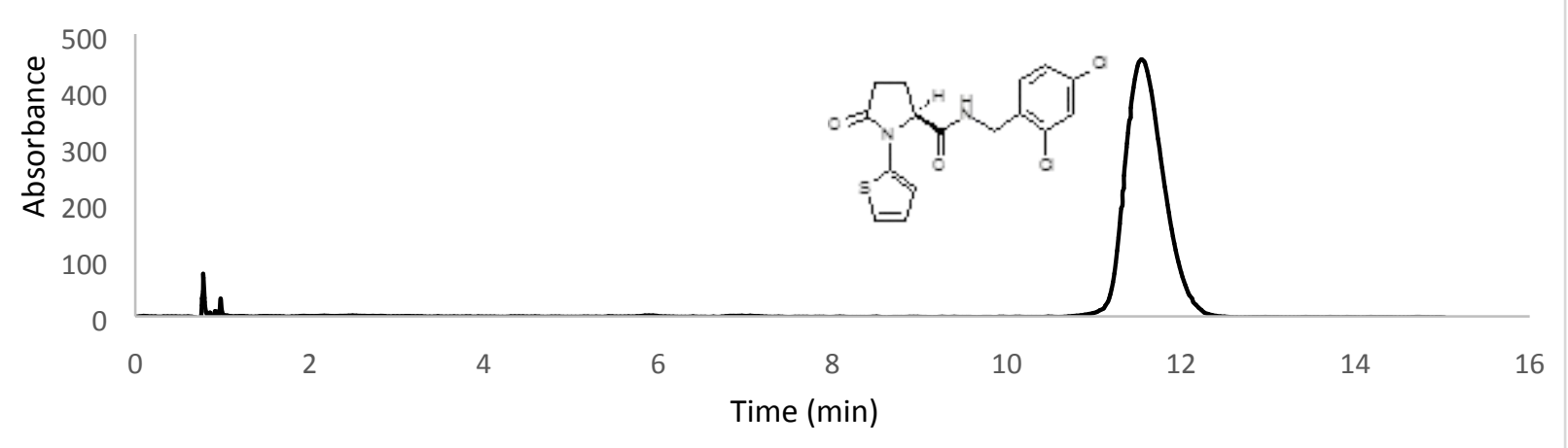

Figure S4-2. Supercritical Fluid Chromatography of Enantiopure Compound 16i resulted in one isomer ( $\left.\mathrm{t}_{\mathrm{R}}, 11.6 \mathrm{~min}\right)$. 


\section{h-P2X7R screening:}

Material and methods

HEK-293 cells overexpressing human P2X7R were stained with the calcium indicator Fluo-3 AM and then incubated with the non-permeant intercalants TO-PRO-3 (large pore opening) and propidium iodide (cell viability) in presence of different molecules at $10^{-5} \mathrm{M}$ concentration for $15 \mathrm{~min}$ before addition of the P2X7R agonist BzATP $(100 \mu \mathrm{M})$. Compounds 1a was used as a reference antagonist for human P2X7R. Cells were then analyzed after 1 hour by flow cytometry using Cyan cytometer (Beckman). Percentages of cells displaying P2X7R activation (Fluo-3/TO-PRO-3 double positive cells) in presence of the molecules were calculated.

$\underline{\text { Results }}$

Results for series I-VI are shown in tables S1-S6.

Table S1. Series I, TO-PRO-3 uptake assay in $h$ P2X7-expressing HEK293 cells

\begin{tabular}{|c|c|c|c|c|}
\hline compd & $\mathrm{R} 1$ & $\mathrm{R} 2$ & $\begin{array}{c}\text { Inhibition of } h- \\
\text { P2X7 at } 10^{-5} \mathrm{M} \\
(\%)^{\mathrm{a}}\end{array}$ & $\mathrm{IC}_{50} \pm \mathrm{SD}(\mathrm{nM})^{\mathrm{a}}$ \\
\hline $1 \mathrm{a}$ & $\mathrm{Me}$ & $\mathrm{CH}_{2}-(2,4-\mathrm{diCl}) \mathrm{Ph}$ & $99.9 \pm 0.3 \%$ & $474 \pm 12.0$ \\
\hline $1 b$ & $\mathrm{Me}$ & $\mathrm{CH}_{2}-\left(2-\mathrm{Cl}-3-\mathrm{CF}_{3}\right) \mathrm{Ph}$ & $98.0 \pm 0.6 \%$ & $119.3 \pm 7.0$ \\
\hline 1c & $\mathrm{Me}$ & $\mathrm{CH}_{2}-\left(2-\mathrm{Cl}-5-\mathrm{CF}_{3}\right) \mathrm{Ph}$ & $0 \%{ }^{b}$ & $\underline{-c}$ \\
\hline 1d & $\mathrm{Me}$ & $\mathrm{CH}\left(\mathrm{CH}_{3}\right)-(2,4-\mathrm{diCl}) \mathrm{Ph}$ & $99.4 \pm 0.5 \%$ & $1305.0 \pm 262.0$ \\
\hline 56 & $\mathrm{CHO}$ & $\mathrm{CH}_{2}-(2,4-\mathrm{diCl}) \mathrm{Ph}$ & $25.4 \pm 3.3 \%$ & ${ }^{c}$ \\
\hline
\end{tabular}

${ }^{a} \%$ Inhibition values at $10 \mu \mathrm{M}$ were expressed as a percentage, relative to maximum incorporation of TO-PRO-3 stimulated by BzATP only, and $\mathrm{IC}_{50}$ values were obtained from concentration-response curves. Data values are expressed as means \pm SDs. All experiments were repeated at least 3 times. ${ }^{b}$ No activity at $10 \mu \mathrm{M}$. ${ }^{c}$ Not determined.

Table S2. Series II, TO-PRO-3 uptake assay in $h \mathrm{P} 2 \mathrm{X} 7$-expressing HEK293

\begin{tabular}{|c|c|c|c|c|c|}
\hline compd & $\mathrm{R}_{1}$ & $\mathrm{R}_{2}$ & $\mathrm{R}_{3}$ & $\begin{array}{c}\text { Inhibition of } h-\mathrm{P} 2 \mathrm{X} 7 \\
\text { at } 10^{-5} \mathrm{M}^{\mathrm{a}}\end{array}$ & $\begin{array}{c}\mathrm{IC}_{50} \pm \mathrm{SD} \\
(\mathrm{nM})^{\mathrm{a}}\end{array}$ \\
\hline 14 & $\mathrm{Ph}$ & $(2,4-\mathrm{diCl}) \mathrm{Ph}$ & $\mathrm{H}$ & $0 \%^{b}$ & ${ }^{c}$ \\
\hline 15 & $\mathrm{Ph}$ & 4-toluyl & $\mathrm{H}$ & $0 \%{ }^{b}$ & ${ }^{c}$ \\
\hline $16 a$ & $\mathrm{Ph}$ & $\mathrm{CH}_{2}-(2,4-\mathrm{diCl}) \mathrm{Ph}$ & $\mathrm{H}$ & $50.0 \pm 0.3 \%$ & $10000.0 \pm 151.1$ \\
\hline $16 \mathrm{~b}$ & $(2-\mathrm{Cl}) \mathrm{Ph}$ & $\mathrm{CH}_{2}-(2,4-\mathrm{diCl}) \mathrm{Ph}$ & $\mathrm{H}$ & $0 \%{ }^{b}$ & ${ }^{c}$ \\
\hline $16 \mathrm{c}$ & $(2,4-\mathrm{diCl}) \mathrm{Ph}$ & $\mathrm{CH}_{2}-(2,4-\mathrm{diCl}) \mathrm{Ph}$ & $\mathrm{H}$ & $0 \%{ }^{b}$ & $c$ \\
\hline 16d & $\left(3-\mathrm{CF}_{3}\right) \mathrm{Ph}$ & $\mathrm{CH}_{2}-(2,4-\mathrm{diCl}) \mathrm{Ph}$ & $\mathrm{H}$ & $0 \%^{b}$ & ${ }_{c}^{c}$ \\
\hline $16 \mathrm{e}$ & $(3,5-\mathrm{diCl}) \mathrm{Ph}$ & $\mathrm{CH}_{2}-(2,4-\mathrm{diCl}) \mathrm{Ph}$ & $\mathrm{H}$ & $0 \%{ }^{b}$ & $c$ \\
\hline $16 f$ & (4-Cl)Ph & $\mathrm{CH}_{2}-(2,4-\mathrm{diCl}) \mathrm{Ph}$ & $\mathrm{H}$ & $0 \%{ }^{b}$ & $c$ \\
\hline $16 \mathrm{~g}$ & $(4-\mathrm{CN}) \mathrm{Ph}$ & $\mathrm{CH}_{2}-(2,4-\mathrm{diCl}) \mathrm{Ph}$ & $\mathrm{H}$ & $0 \%{ }^{b}$ & ${ }^{c}$ \\
\hline $16 \mathrm{~h}$ & (4-MeO)Ph & $\mathrm{CH}_{2}-(2,4-\mathrm{diCl}) \mathrm{Ph}$ & $\mathrm{H}$ & $0 \%^{b}$ & ]$^{c}$ \\
\hline $16 \mathbf{i}$ & 2-thienyl & $\mathrm{CH}_{2}-(2,4-\mathrm{diCl}) \mathrm{Ph}$ & $\mathrm{H}$ & $98.2 \pm 14.3 \%$ & $229 . \overline{0} \pm 4.5$ \\
\hline $16 \mathrm{j}$ & 3-thienyl & $\mathrm{CH}_{2}-(2,4-\mathrm{diCl}) \mathrm{Ph}$ & $\mathrm{H}$ & $78.3 \pm 14.2 \%$ & $813.2 \pm 56.7$ \\
\hline $16 k$ & 2-furanyl & $\mathrm{CH}_{2}-(2,4-\mathrm{diCl}) \mathrm{Ph}$ & $\mathrm{H}$ & $94.4 \pm 2.4 \%$ & $244.0 \pm 105.0$ \\
\hline 161 & 3-furanyl & $\mathrm{CH}_{2}-(2,4-\mathrm{diCl}) \mathrm{Ph}$ & $\mathrm{H}$ & $84.7 \pm 9.4 \%$ & $199.5 \pm 15.5$ \\
\hline $16 \mathrm{~m}$ & 2-thiazolyl & $\mathrm{CH}_{2}-(2,4-\mathrm{diCl}) \mathrm{Ph}$ & $\mathrm{H}$ & $67.9 \pm 8.7 \%$ & $415.0 \pm 85.0$ \\
\hline $16 n$ & 4-thiazolyl & $\mathrm{CH}_{2}-(2,4-\mathrm{diCl}) \mathrm{Ph}$ & $\mathrm{H}$ & $75.9 \pm 23.6 \%$ & $256.4 \pm 1.0$ \\
\hline 160 & 5-thazolyl & $\mathrm{CH}_{2}-(2,4-\mathrm{diCl}) \mathrm{Ph}$ & $\mathrm{H}$ & $62.1 \pm 9.1 \%$ & $c$ \\
\hline $16 p$ & 4-pyridyl & $\mathrm{CH}_{2}-(2,4-\mathrm{diCl}) \mathrm{Ph}$ & $\mathrm{H}$ & $0 \%^{b}$ & $c$ \\
\hline $16 q$ & 5-quinolinyl & $\mathrm{CH}_{2}-(2,4-\mathrm{diCl}) \mathrm{Ph}$ & $\mathrm{H}$ & $0 \%{ }^{b}$ & $c$ \\
\hline 17 & $\mathrm{Ph}$ & $\mathrm{CH}_{2}$-cyclohexyl & $\mathrm{H}$ & $0 \%{ }^{b}$ & $c$ \\
\hline 18 & $\mathrm{Ph}$ & $\mathrm{CH}_{2}-(2-\mathrm{Cl}) \mathrm{Ph}$ & $\mathrm{H}$ & $0 \%^{b}$ & ${ }^{c}$ \\
\hline 19 & 2-thienyl & $\mathrm{CH}_{2}-\left(2-\mathrm{Cl}-3-\mathrm{CF}_{3}\right) \mathrm{Ph}$ & $\mathrm{H}$ & $99.9 \pm 1.0 \%$ & $100 . \overline{0} \pm 10.1$ \\
\hline 20 & 2-thienyl & $\mathrm{CH}_{2}-\left(2-\mathrm{Cl}-5-\mathrm{CF}_{3}\right) \mathrm{Ph}$ & $\mathrm{H}$ & $17.5 \pm 5.7 \%$ & $\underline{c}$ \\
\hline 21 & 2-thienyl & $\mathrm{CH}\left(\mathrm{CH}_{3}\right)-(2,4-\mathrm{diCl}) \mathrm{Ph}$ & $\mathrm{H}$ & $0 \%^{b}$ & $c$ \\
\hline 22 & $\mathrm{Ph}$ & $\mathrm{Me}$ & $\mathrm{Me}$ & $0 \%^{b}$ & $c$ \\
\hline 23 & $\mathrm{Ph}$ & $\mathrm{CH}_{2}-\mathrm{CH}_{2}-(2,4-\mathrm{diCl}) \mathrm{Ph}$ & $\mathrm{H}$ & $0 \%^{b}$ & $c$ \\
\hline
\end{tabular}

$a \%$ Inhibition values at $10 \mu \mathrm{M}$ were expressed as a percentage, relative to maximum incorporation of TO-PRO-3 stimulated by BzATP only, and $\mathrm{IC}_{50}$ values were obtained from concentration-response curves. Data values are expressed as means \pm SDs. All experiments were repeated at least 3 times. ${ }^{b}$ No activity at $10 \mu \mathrm{M}$. ${ }^{c}$ Not determined. 
Table S3. Series III, TO-PRO-3 uptake assay in $h \mathrm{P} 2 \mathrm{X} 7$-expressing HEK293

\begin{tabular}{|c|c|c|c|c|c|c|c|}
\hline compd & $\mathrm{n}$ & $\mathrm{R}_{1}$ & $\mathrm{R}_{2}$ & $\mathrm{R}_{3}$ & $\mathrm{R}_{4}$ & $\begin{array}{l}\text { Inhibition of } h- \\
\mathrm{P} 2 \mathrm{X} 7 \text { at } 10^{-5} \mathrm{M}^{\mathrm{a}}\end{array}$ & $\mathrm{IC}_{50} \pm \mathrm{SD}(\mathrm{nM})^{\mathrm{a}}$ \\
\hline 28 & 0 & $(2-\mathrm{Cl}) \mathrm{Ph}$ & $\mathrm{H}$ & $\mathrm{H}$ & $(2,4-\mathrm{diCl}) \mathrm{Ph}$ & $0 \%^{b}$ & $c$ \\
\hline 29a & 1 & $\mathrm{Ph}$ & $\mathrm{H}$ & $\mathrm{H}$ & $\mathrm{Ph}$ & $0 \%^{b}$ & $c$ \\
\hline $29 b$ & 1 & $\mathrm{Ph}$ & $\mathrm{H}$ & $\mathrm{H}$ & $(2-\mathrm{Cl}) \mathrm{Ph}$ & $0 \%^{b}$ & $\tau^{c}$ \\
\hline $29 \mathrm{c}$ & 1 & $\mathrm{Ph}$ & $\mathrm{H}$ & $\mathrm{H}$ & $(2,4-\mathrm{diCl}) \mathrm{Ph}$ & $99.6 \pm 0.6 \%$ & $1130 . \overline{3 \pm} 120.2$ \\
\hline 30a & 1 & $(2-\mathrm{Cl}) \mathrm{Ph}$ & $\mathrm{H}$ & $\mathrm{H}$ & $(2-\mathrm{Cl}) \mathrm{Ph}$ & $0 \%^{b}$ & ${ }^{c}$ \\
\hline 30b & 1 & $(2-\mathrm{Cl}) \mathrm{Ph}$ & $\mathrm{H}$ & $\mathrm{H}$ & $(2,4-\mathrm{diCl}) \mathrm{Ph}$ & $85.4 \pm 0.8 \%$ & $3461.2 \pm 523.0$ \\
\hline 31a & 1 & $(4-\mathrm{Cl}) \mathrm{Ph}$ & $\mathrm{H}$ & $\mathrm{H}$ & $(2-\mathrm{Cl}) \mathrm{Ph}$ & $0 \%^{b}$ & c \\
\hline 31b & 1 & (4-Cl)Ph & $\mathrm{H}$ & $\mathrm{H}$ & $(2,4-\mathrm{diCl}) \mathrm{Ph}$ & $0 \%^{b}$ & $c$ \\
\hline $32 a$ & 1 & $(2,4-\mathrm{diCl}) \mathrm{Ph}$ & $\mathrm{H}$ & $\mathrm{H}$ & $\mathrm{Ph}$ & $0 \%^{b}$ & $c$ \\
\hline $32 \mathrm{~b}$ & 1 & $(2,4-\mathrm{diCl}) \mathrm{Ph}$ & $\mathrm{H}$ & $\mathrm{H}$ & $(2-\mathrm{Cl}) \mathrm{Ph}$ & $0 \%^{b}$ & $c$ \\
\hline $32 \mathrm{c}$ & 1 & (2,4-diCl)Ph & $\mathrm{H}$ & $\mathrm{H}$ & $(2,4-\mathrm{diCl}) \mathrm{Ph}$ & $0 \%^{b}$ & ${ }^{c}$ \\
\hline 32d & 1 & $(2,4-\mathrm{diCl}) \mathrm{Ph}$ & $\mathrm{H}$ & $\mathrm{H}$ & $(2,4-\mathrm{diMeO}) \mathrm{Ph}$ & $0 \%^{b}$ & $c$ \\
\hline $32 \mathrm{e}$ & 1 & $(2,4-\mathrm{diCl}) \mathrm{Ph}$ & $\mathrm{H}$ & $\mathrm{H}$ & 2-furanyl & $0 \%^{b}$ & $c$ \\
\hline 33 & 1 & $\left(4-\mathrm{NO}_{2}\right) \mathrm{Ph}$ & $\mathrm{H}$ & $\mathrm{H}$ & $(2,4-\mathrm{diCl}) \mathrm{Ph}$ & $0 \%^{b}$ & ${ }^{c}$ \\
\hline 34 & 1 & $(2,4-\mathrm{diCl}) \mathrm{Ph}$ & $\mathrm{H}$ & $\mathrm{Me}$ & $\mathrm{Ph}$ & $0 \%^{b}$ & $c$ \\
\hline 35 & 1 & $\mathrm{Ph}$ & $\mathrm{Ph}$ & $\mathrm{H}$ & $(2,4-\mathrm{diCl}) \mathrm{Ph}$ & $0 \%^{b}$ & ${ }^{c}$ \\
\hline 36 & 1 & $(4-\mathrm{Cl}) \mathrm{Ph}$ & (4-Cl)Ph & $\mathrm{H}$ & $(2,4-\mathrm{diCl}) \mathrm{Ph}$ & $0 \%^{b}$ & ${ }^{c}$ \\
\hline 37 & 2 & $\mathrm{Ph}$ & $\mathrm{H}$ & $\mathrm{H}$ & $\mathrm{Ph}$ & $0 \%^{b}$ & ${ }^{c}$ \\
\hline 38 & 2 & (4-Cl)Ph & $\mathrm{H}$ & $\mathrm{H}$ & $\mathrm{Ph}$ & $0.1 \pm 0.6 \%$ & $-c$ \\
\hline 39 & 2 & $(4-\mathrm{Br}) \mathrm{Ph}$ & $\mathrm{H}$ & $\mathrm{H}$ & $\mathrm{Ph}$ & $2.9 \pm 4.0 \%$ & ${ }_{c}$ \\
\hline 40 & 2 & $(4-\mathrm{Me}) \mathrm{Ph}$ & $\mathrm{H}$ & $\mathrm{H}$ & $\mathrm{Ph}$ & $0 \%^{b}$ & $c$ \\
\hline 41 & 2 & $(4-\mathrm{MeO}) \mathrm{Ph}$ & $\mathrm{H}$ & $\mathrm{H}$ & $\mathrm{Ph}$ & $0 \%^{b}$ & $c$ \\
\hline 42 & 2 & $(2,4-\mathrm{diCl}) \mathrm{Ph}$ & $\mathrm{H}$ & $\mathrm{H}$ & $\mathrm{Ph}$ & $0 \%^{b}$ & ${ }^{c}$ \\
\hline 43 & 2 & $\left(4-\mathrm{NO}_{2}\right) \mathrm{Ph}$ & $\mathrm{H}$ & $\mathrm{H}$ & $\mathrm{Ph}$ & $0 \%^{b}$ & ${ }^{c}$ \\
\hline
\end{tabular}

a\% Inhibition values at $10 \mu \mathrm{M}$ were expressed as a percentage, relative to maximum incorporation of TO-PRO-3 stimulated by BzATP only, and $\mathrm{IC}_{50}$ values were obtained from concentration-response curves. Data values are expressed as means \pm SDs. All experiments were repeated at least 3 times. ${ }^{b}$ No activity at $10 \mu \mathrm{M}$. ${ }^{c}$ Not determined.

Table S4. Series IV, TO-PRO-3 uptake assay in $h$ P2X7-expressing HEK293

\begin{tabular}{|c|c|c|c|c|c|c|}
\hline compd & $\mathrm{n}$ & R1 & $\mathrm{R} 2$ & Z & $\begin{array}{l}\text { Inhibition of } h- \\
\mathrm{P} 2 \mathrm{X} 7 \text { at } 10^{-5} \mathrm{M}^{\mathrm{a}}\end{array}$ & $\mathrm{IC}_{50} \pm \mathrm{SD}(\mathrm{nM})^{\mathrm{a}}$ \\
\hline 44 & 1 & 2-thienyl & $\mathrm{NH}-\mathrm{CH}_{2}-(2,4-\mathrm{diCl}) \mathrm{Ph}$ & $\mathrm{CH}-\mathrm{NMe}_{2}$ & $9.8 \pm 21.9 \%$ & $\underline{-}^{c}$ \\
\hline 45 & 1 & 2-thienyl & $\mathrm{NH}-\mathrm{CH}_{2}-(2,4-\mathrm{diCl}) \mathrm{Ph}$ & $\mathrm{N}-\mathrm{OH}$ & $76.7 \pm 22.6 \%$ & $3724.0 \pm 943.0$ \\
\hline 47 & 1 & $\mathrm{CO}_{2} \mathrm{Me}$ & $\mathrm{OMe}$ & $\mathrm{CH}-\mathrm{NH}-\mathrm{CH}_{2}-(2,4-\mathrm{diCl}) \mathrm{Ph}$ & $98.7 \pm 1.7 \%$ & $4267.0 \pm 885.0$ \\
\hline 48 & 1 & $\mathrm{H}$ & $\mathrm{NH}-\mathrm{CH}_{2}-(2,4-\mathrm{diCl}) \mathrm{Ph}$ & $\mathrm{CH}-\mathrm{NH}-\mathrm{CH}_{2}-(2,4-\mathrm{diCl}) \mathrm{Ph}$ & $0 \%^{b}$ & $\underline{c}$ \\
\hline 51a & 1 & $\mathrm{H}$ & $\mathrm{NH}-(4-\mathrm{MeO}) \mathrm{Ph}$ & $\mathrm{CH}-\mathrm{NMe}_{2}$ & $0 \%{ }^{b}$ & $-c$ \\
\hline $51 \mathrm{~b}$ & 1 & $\mathrm{H}$ & $\mathrm{NH}-\mathrm{CH}_{2}-(2-\mathrm{MeO}) \mathrm{Ph}$ & $\mathrm{CH}-\mathrm{NMe}_{2}$ & $0 \%{ }^{b}$ & $-c$ \\
\hline 51c & 1 & $\mathrm{H}$ & $\mathrm{NH}-\mathrm{CH}_{2}-(2,4-\mathrm{diCl}) \mathrm{Ph}$ & $\mathrm{CH}-\mathrm{NMe}_{2}$ & $53.3 \pm 17.4 \%$ & $-c$ \\
\hline (S)51d & 1 & $\mathrm{H}$ & $\mathrm{NH}-\mathrm{CH}\left(\mathrm{CH}_{3}\right)-\mathrm{Ph}$ & $\mathrm{CH}-\mathrm{NMe}_{2}$ & $0 \%^{b}$ & ${ }^{c}$ \\
\hline (R)51d & 1 & $\mathrm{H}$ & $\mathrm{NH}-\mathrm{CH}\left(\mathrm{CH}_{3}\right)-\mathrm{Ph}$ & $\mathrm{CH}-\mathrm{NMe}_{2}$ & $0 \%^{b}$ & $-c$ \\
\hline $52 a$ & 1 & $\mathrm{H}$ & $\mathrm{NH}-\mathrm{CH}_{2}-(2,4-\mathrm{diCl}) \mathrm{Ph}$ & CH-morpholinyl & $56.5 \pm 11.3 \%$ & $9557.0 \pm 756.0$ \\
\hline $52 b$ & 1 & $\mathrm{H}$ & $\mathrm{NH}-\mathrm{CH}_{2}-(2,4-\mathrm{diCl}) \mathrm{Ph}$ & CH-(4-methyl)piperazyl & $49.2 \pm 5.0 \%$ & $6947.0 \pm 486.0$ \\
\hline $52 \mathrm{c}$ & 1 & $\mathrm{H}$ & $\mathrm{NH}-\mathrm{CH}_{2}-(2,4-\mathrm{diCl}) \mathrm{Ph}$ & CH-NH-benzo-(15-crown-5)-yl & $31.4 \pm 10.8 \%$ & $\underline{-c}$ \\
\hline 52d & 1 & $\mathrm{H}$ & $\mathrm{NH}-\mathrm{CH}_{2}-(2,4-\mathrm{diCl}) \mathrm{Ph}$ & $\mathrm{CH}-\mathrm{NH}-\mathrm{CH}_{2}-(3,4,5$-triMeO)Ph & $16.2 \pm 1.6 \%$ & $-c$ \\
\hline 53 & 1 & $\mathrm{H}$ & $\mathrm{NH}-\mathrm{CH}_{2}-(2,4-\mathrm{diCl}) \mathrm{Ph}$ & $\mathrm{N}-\mathrm{OH}$ & $52.0 \pm 0.7 \%$ & ${ }^{c}$ \\
\hline 54 & 1 & $\mathrm{H}$ & $\mathrm{NH}-\mathrm{CH}_{2}-(2,4-\mathrm{diCl}) \mathrm{Ph}$ & $\mathrm{CH}-\mathrm{OH}$ & $48.3 \pm 3.8 \%$ & ${ }^{c}$ \\
\hline 71 & 2 & $\mathrm{H}$ & $\mathrm{NH}-\mathrm{CH}_{2}-(2,4-\mathrm{diCl}) \mathrm{Ph}$ & $\mathrm{CH}-\mathrm{NMe}_{2}$ & $5.9 \pm 5.8 \%$ & ${ }^{c}$ \\
\hline
\end{tabular}

a\% Inhibition values at $10 \mu \mathrm{M}$ were expressed as a percentage, relative to maximum incorporation of TO-PRO-3 stimulated by BzATP only, and $\mathrm{IC}_{50}$ values were obtained from concentration-response curves. Data values are expressed as means \pm SDs. All experiments were repeated at least 3 times. ${ }^{b}$ No activity at $10 \mu \mathrm{M}$. ${ }^{c}$ Not determined. 
Table S5. Series V, TO-PRO-3 uptake assay in $h \mathrm{P} 2 \mathrm{X} 7$-expressing HEK293

\begin{tabular}{|c|c|c|c|}
\hline compd & $\mathrm{R}_{1}$ & Z & $\begin{array}{l}\text { Inhibition of } h- \\
\text { P2X7 at } 10^{-5} \mathrm{M}^{\text {a }}\end{array}$ \\
\hline 49 & $n$-hexyl & - & $0 \%^{b}$ \\
\hline $50 a$ & $n$-hexyl & $\mathrm{CH}-\mathrm{NMe}_{2}$ & $0 \%^{b}$ \\
\hline $50 \mathrm{~b}$ & $(3,4,5$-triMeO)Ph & $\mathrm{CH}-\mathrm{NMe}_{2}$ & $0 \%^{b}$ \\
\hline 50c & $(4-\mathrm{MeO}) \mathrm{Ph}$ & $\mathrm{CH}-\mathrm{NMe}_{2}$ & $0 \%^{b}$ \\
\hline 50d & $\mathrm{CH}_{2}-\mathrm{Ph}$ & $\mathrm{CH}-\mathrm{NMe}_{2}$ & $0 \%^{b}$ \\
\hline $50 e$ & $\mathrm{CH}_{2}-(2-\mathrm{Cl}) \mathrm{Ph}$ & $\mathrm{CH}-\mathrm{NMe}_{2}$ & $3.6 \pm 0.6 \%$ \\
\hline $50 \mathrm{f}$ & $\mathrm{CH}_{2}-(2-\mathrm{MeO}) \mathrm{Ph}$ & $\mathrm{CH}-\mathrm{NMe}_{2}$ & $3.0 \pm 0.9 \%$ \\
\hline $50 \mathrm{~g}$ & $\mathrm{CH}_{2}-(2,4-\mathrm{diCl}) \mathrm{Ph}$ & $\mathrm{CH}-\mathrm{NMe}_{2}$ & $23.7 \pm 13.6 \%$ \\
\hline $50 \mathrm{~h}$ & $\mathrm{CH}_{2}-(2,4-\mathrm{diMeO}) \mathrm{Ph}$ & $\mathrm{CH}-\mathrm{NMe}_{2}$ & $0 \%^{b}$ \\
\hline (S)50i & $\mathrm{CH}\left(\mathrm{CH}_{3}\right)-\mathrm{Ph}$ & $\mathrm{CH}-\mathrm{NMe}_{2}$ & $0 \%^{b}$ \\
\hline (R)50i & $\mathrm{CH}\left(\mathrm{CH}_{3}\right)-\mathrm{Ph}$ & $\mathrm{CH}-\mathrm{NMe}_{2}$ & $37.2 \pm 1.2 \%$ \\
\hline $\mathbf{5 0 j}$ & $\mathrm{CH}_{2}-\mathrm{CH}_{2}-\mathrm{Ph}$ & $\mathrm{CH}-\mathrm{NMe}_{2}$ & $0 \%^{b}$ \\
\hline $50 \mathrm{k}$ & $\mathrm{C}\left(\mathrm{CHNMe}_{2}\right)$-(4-Pyridyl) & $\mathrm{CH}-\mathrm{NMe}_{2}$ & $0 \%^{b}$ \\
\hline 55 & $\mathrm{CH}_{2}-(2,4-\mathrm{diCl}) \mathrm{Ph}$ & $\mathrm{O}$ & $0 \%^{b}$ \\
\hline
\end{tabular}

$a \%$ Inhibition values at $10 \mu \mathrm{M}$ were expressed as a percentage, relative to maximum incorporation of TO-PRO-3 stimulated by BzATP only, and $\mathrm{IC}_{50}$ values were obtained from concentration-response curves. Data values are expressed as means \pm SDs. All experiments were repeated at least 3 times. ${ }^{b}$ No activity at $10 \mu \mathrm{M}$.

Table S6. Series VI, TO-PRO-3 uptake assay in $h$ P2X7-expressing HEK293

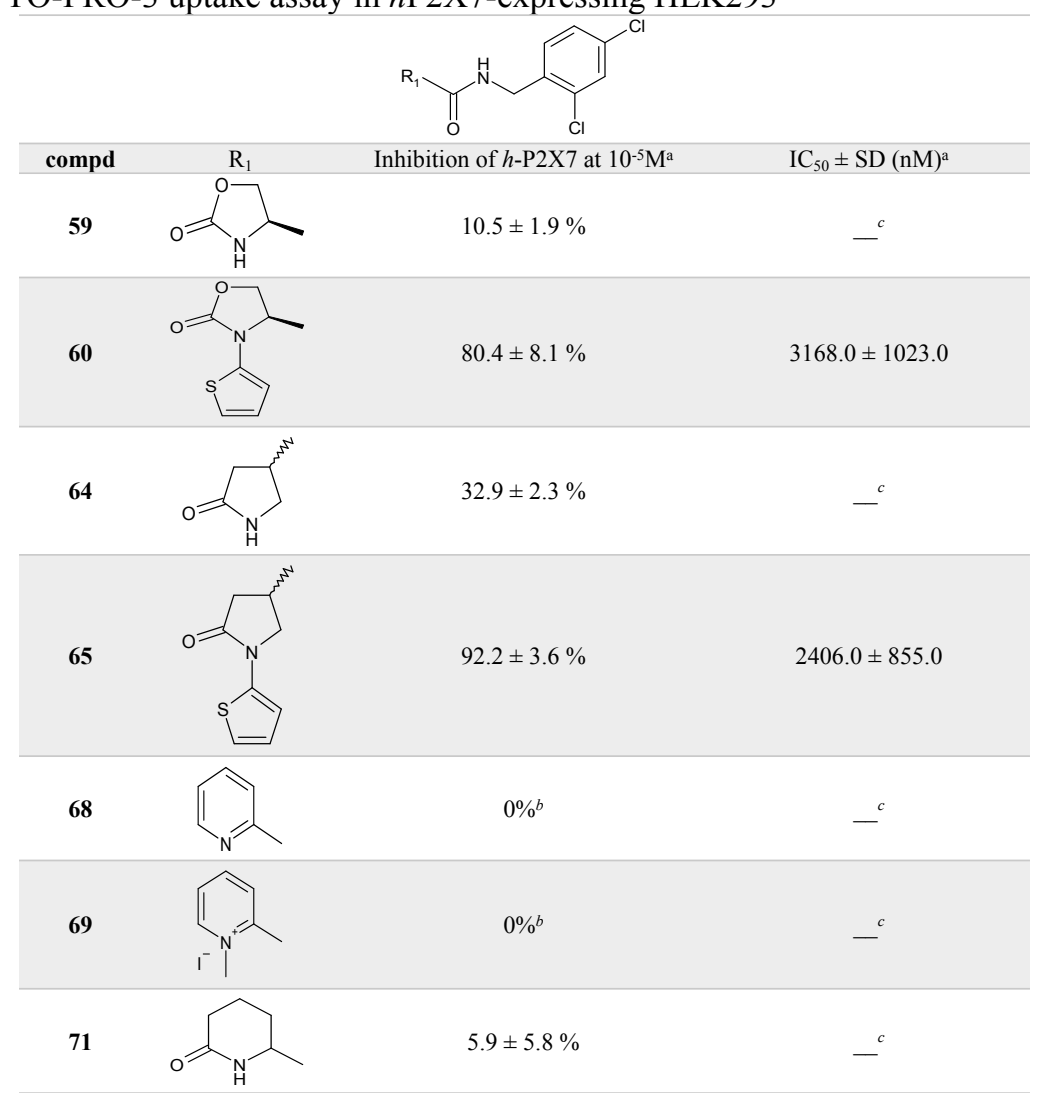

a\% Inhibition values at $10 \mu \mathrm{M}$ were expressed as a percentage, relative to maximum incorporation of TO-PRO-3 stimulated by BzATP only, and $\mathrm{IC}_{50}$ values were obtained from concentration-response curves. Data values are expressed as means \pm SDs. All experiments were repeated at least 3 times. ${ }^{b}$ No activity at $10 \mu \mathrm{M}$. ${ }^{c}$ Not determined. 


\section{Molecular docking of P2X7 antagonists:}

$P d$-P2X7 was used as structural template for docking studies as sequence is totally conserved into region corresponding to allosteric binding site. Among the 6 experimental structures of $p d-\mathrm{P} 2 \mathrm{X} 7$ co-crystallized with different reference allosteric antagonists, we chose the 5U1X PDB entry co-crystallized with JNJ47965567 exhibiting the highest structural similarity with the studied chemical series as low-weighted and coiled structures. Molecular docking jobs were performed with AutodockVina 1.1.2, ${ }^{28}$ using the 5U1X homotrimeric P2X7 structure with 2 of 3 allosteric binding sites occupied and centering a large searching box that includes the whole extracellular trimeric region in order to assess the specificity for allosteric binding site. Receptor-ligand interaction was derived from a pKd calculated from the Vina score, a standard free energy of binding $\left(\Delta G_{b i n d}^{0}\right)$ associated to each binding docking pose, and according to the following thermodynamic relationship:

$$
\begin{gathered}
\Delta G_{\text {bind }}^{0}=-R T \ln K_{e q}=-2,303 \cdot R T \log _{10} K_{e q} \\
p K_{d}=\frac{\Delta G_{b i n d}^{0}}{-2,303 \cdot R T}
\end{gathered}
$$

Figures S2-S13 are showing superimposition of docking poses of selected P2X7 potential antagonists of this study.

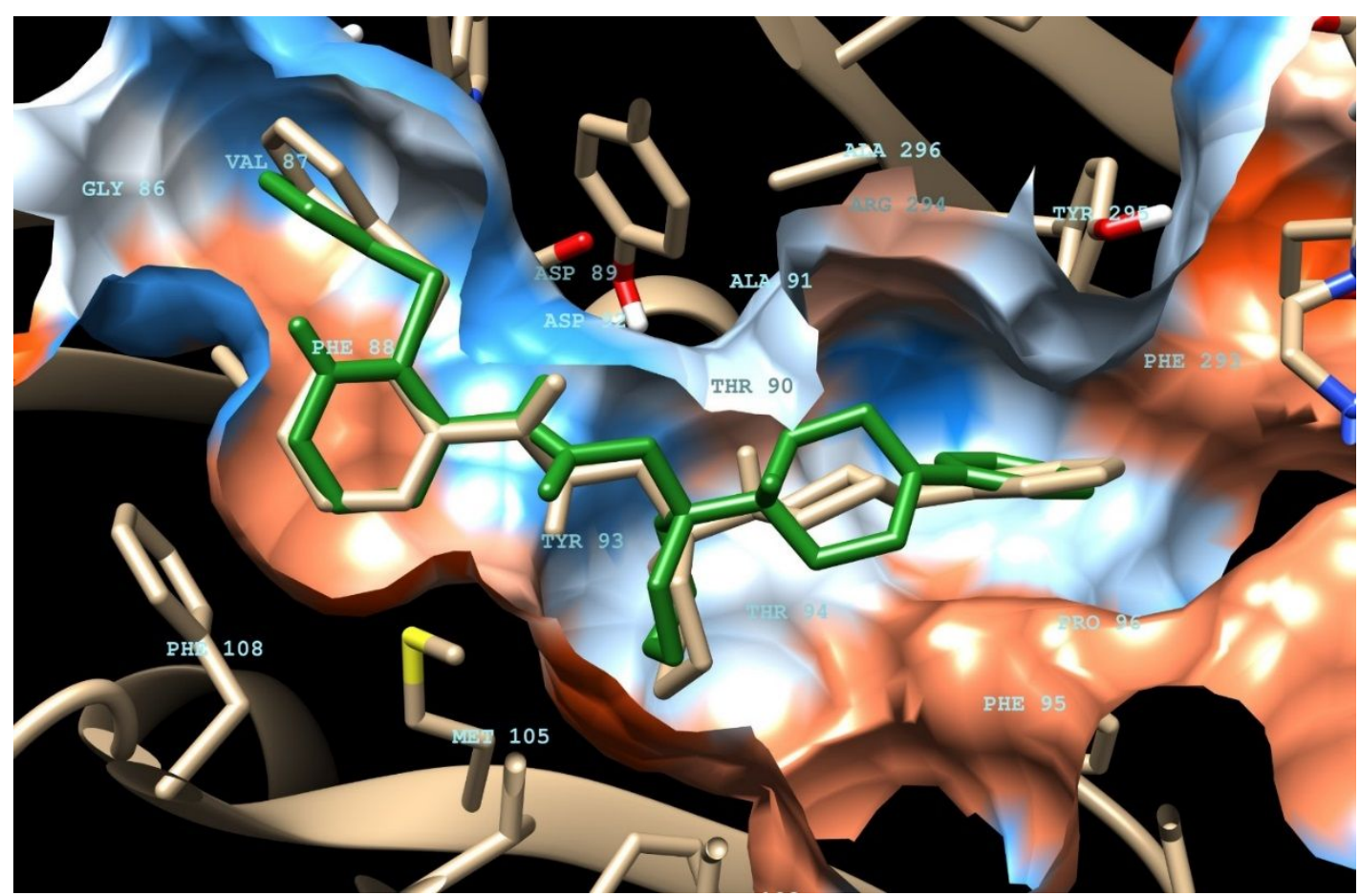

Figure S2. JNJ47965567 experimental pose (grey) vs JNJ47965567predicted docking pose (green). 


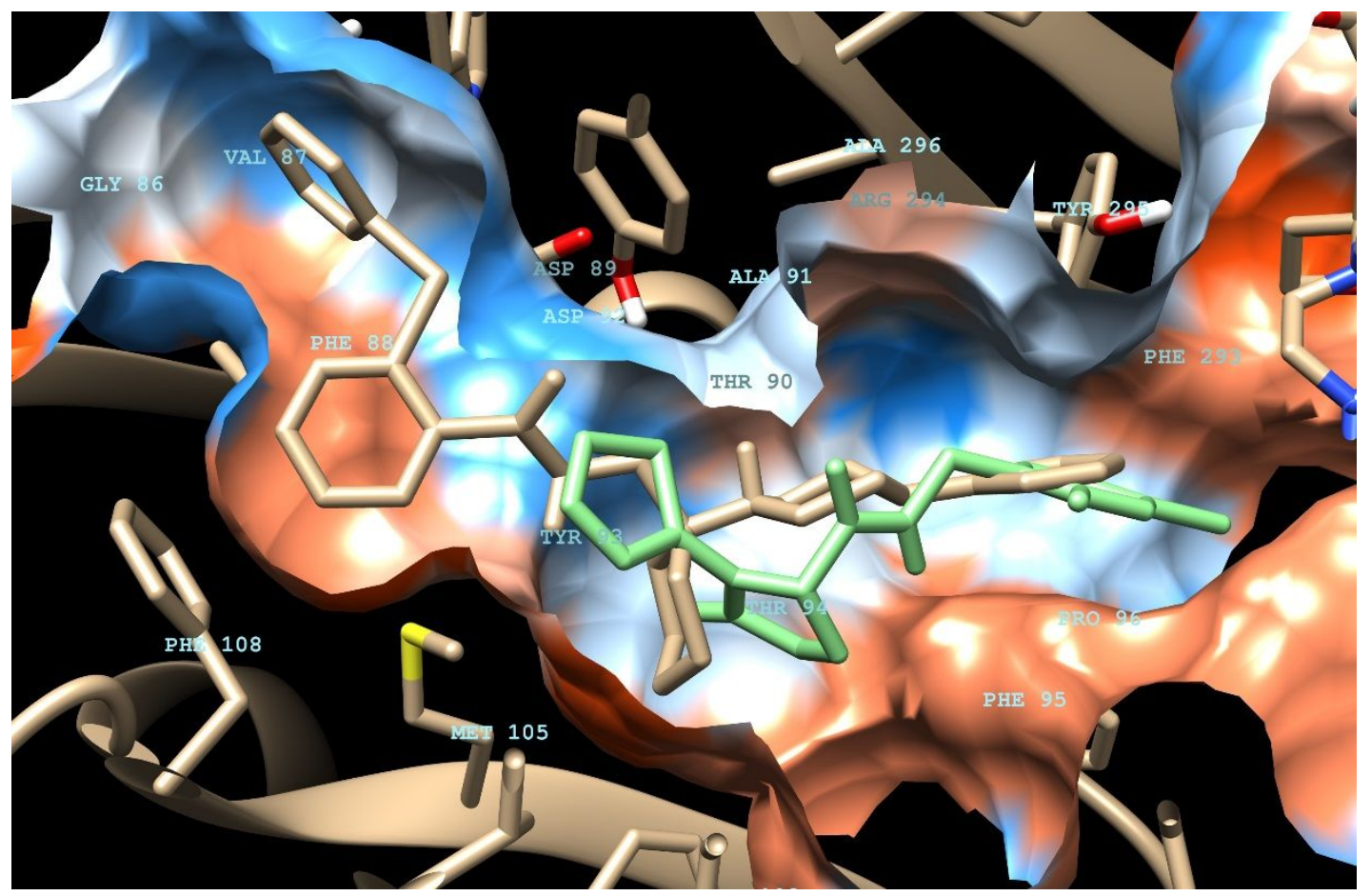

Figure S3. JNJ47965567 experimental pose (grey) vs docking pose predicted for 16i (light green).

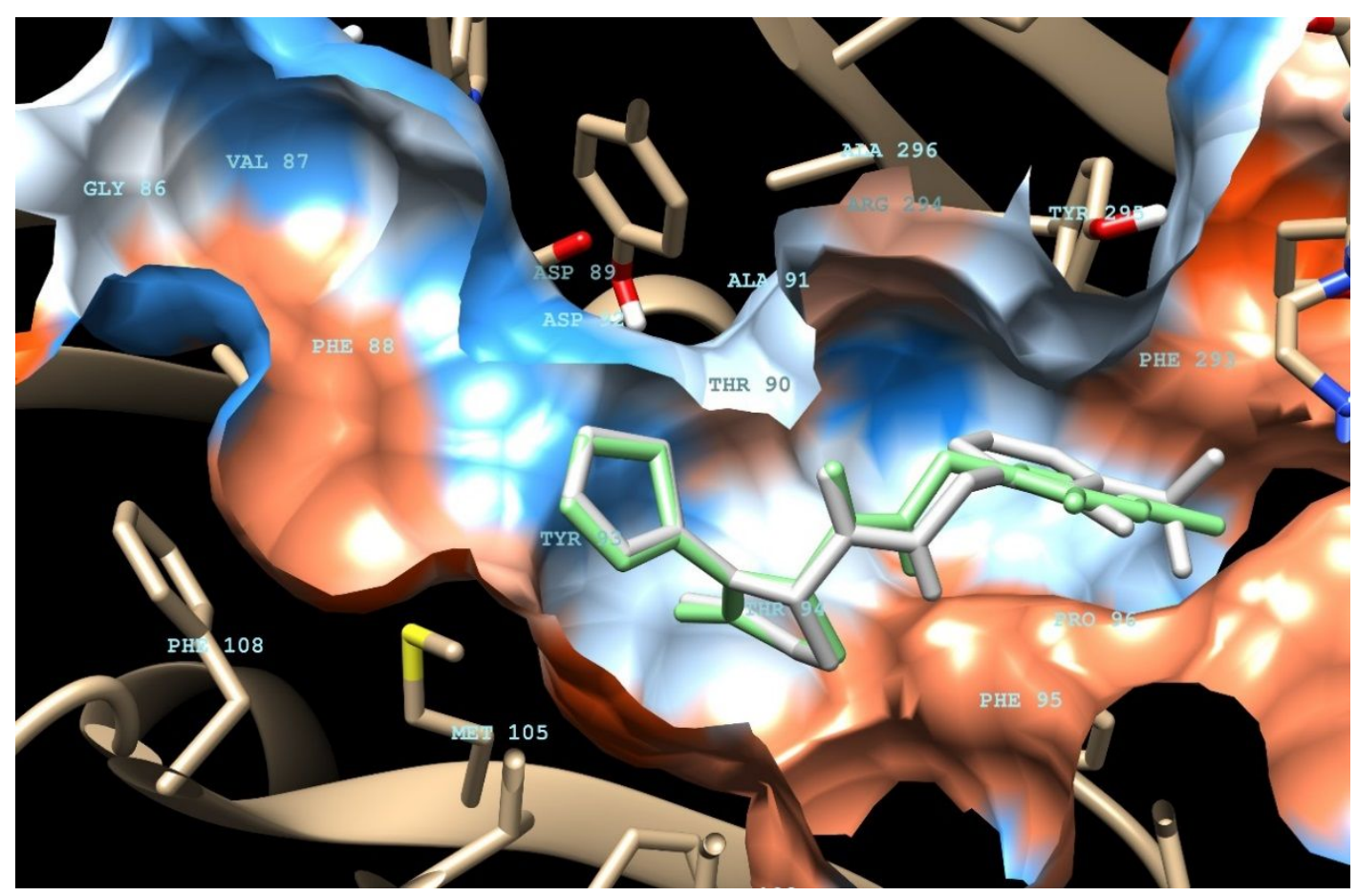

Figure S4. Docking poses predicted for $\mathbf{1 6}$ (light green) vs $\mathbf{1 9}$ (white). 


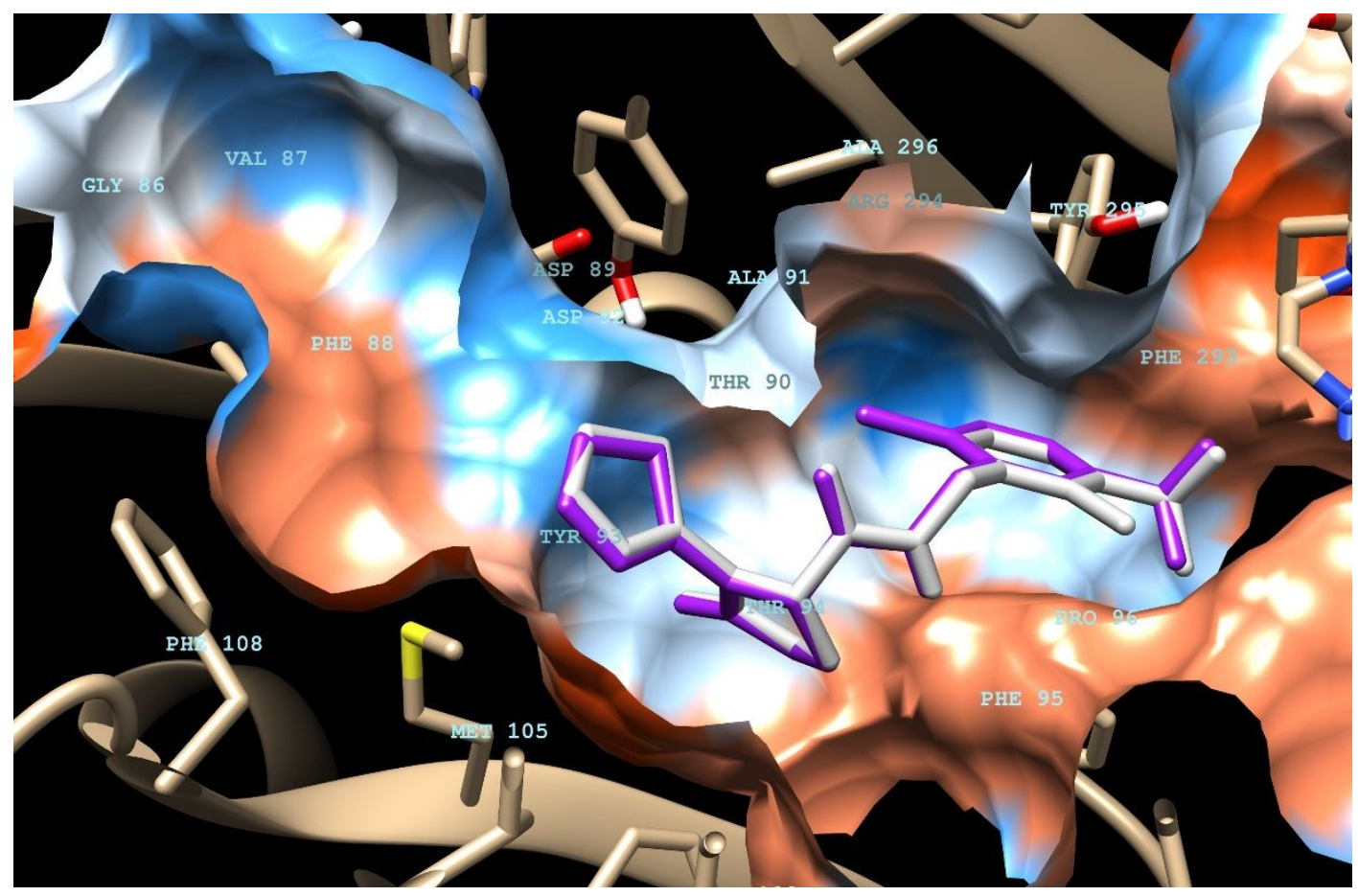

Figure S4. Docking poses predicted for $\mathbf{1 9}$ (white) vs $\mathbf{2 0}$ (pink).

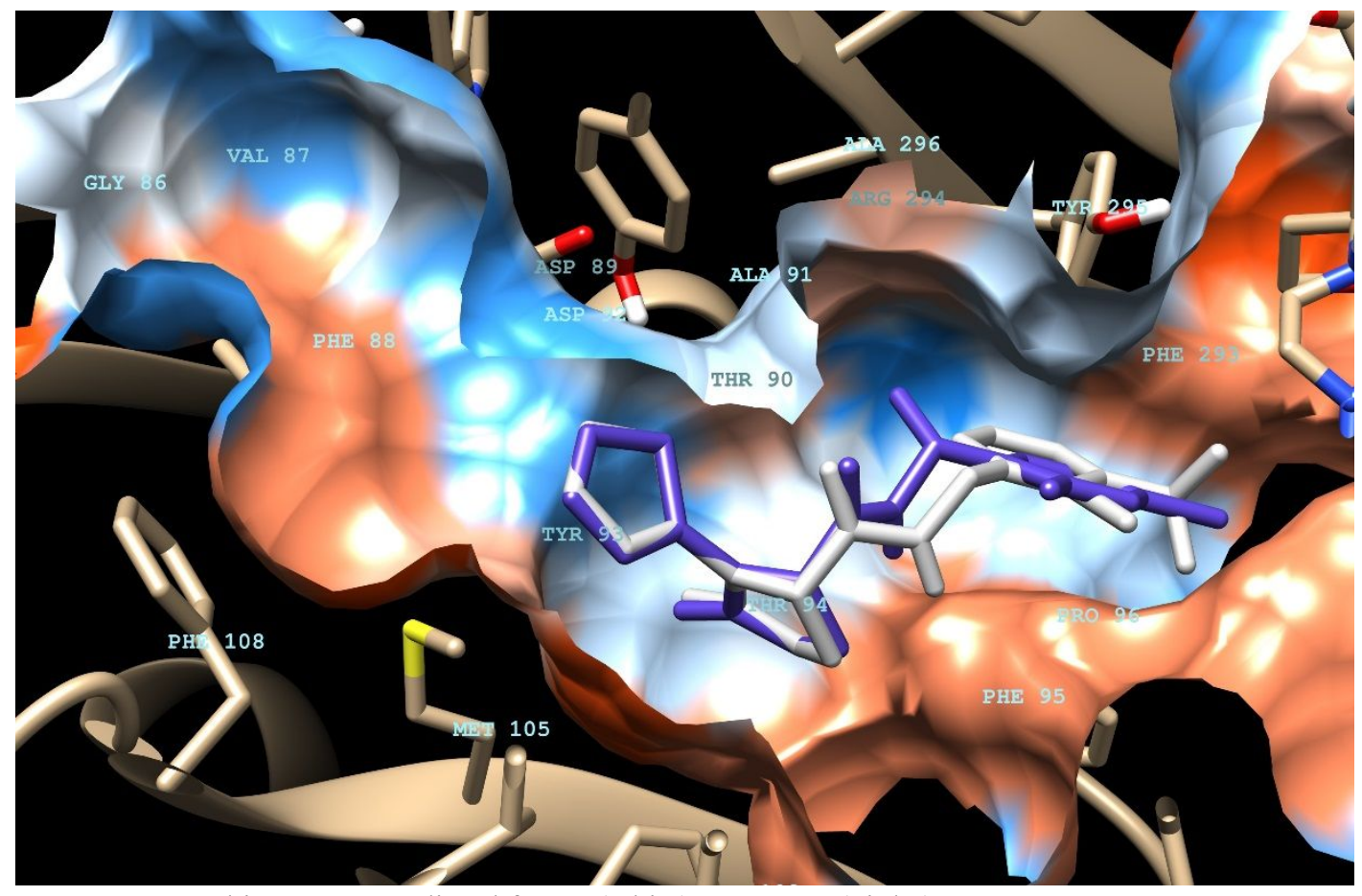

Figure S6. Docking poses predicted for 19 (white) vs (R)-21 (violet). 


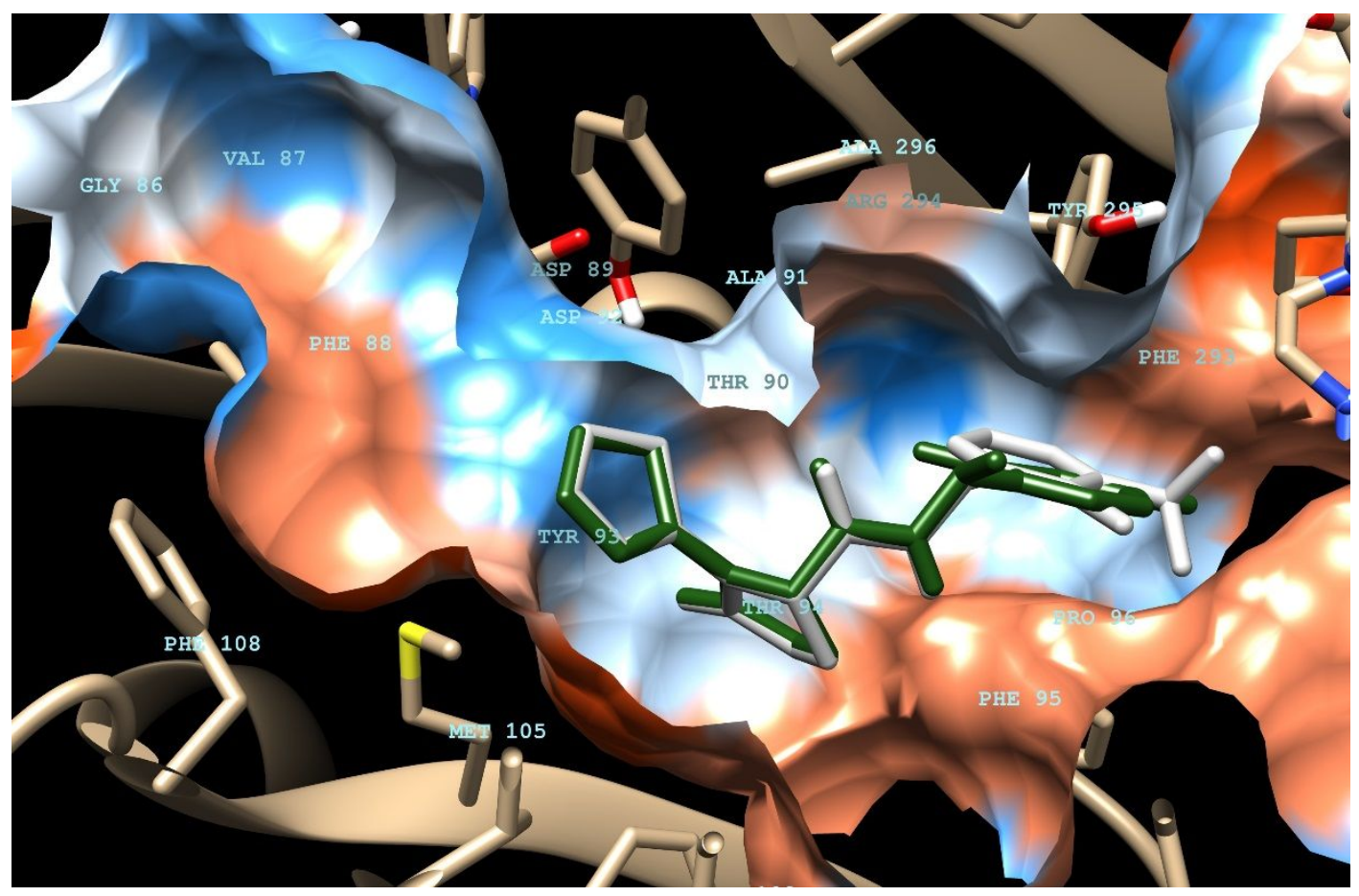

Figure S7. Docking poses predicted for 19 (white) vs (S)-21 (green).

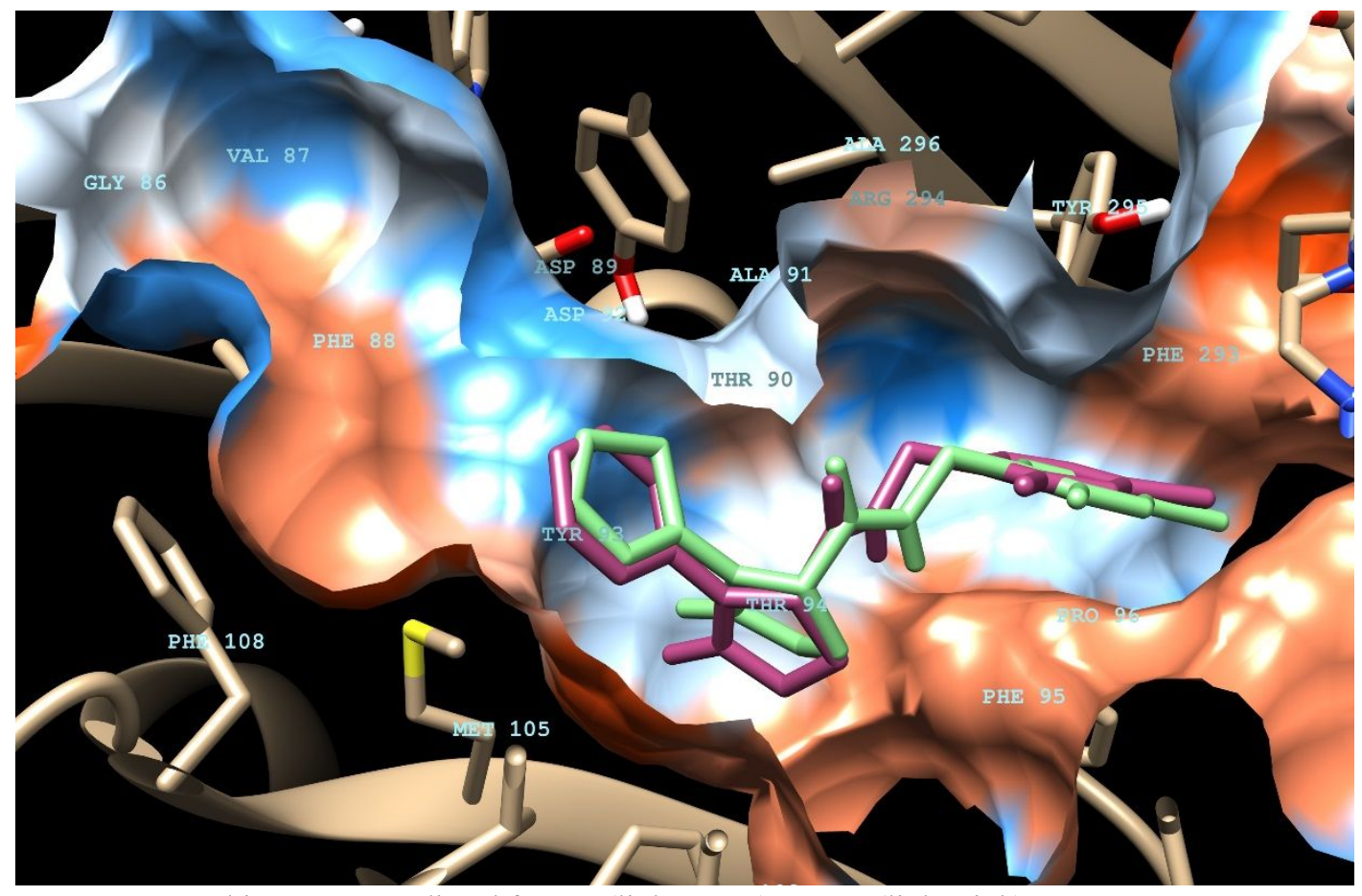

Figure S8. Docking poses predicted for 16i (light green) vs 16a (light pink). 


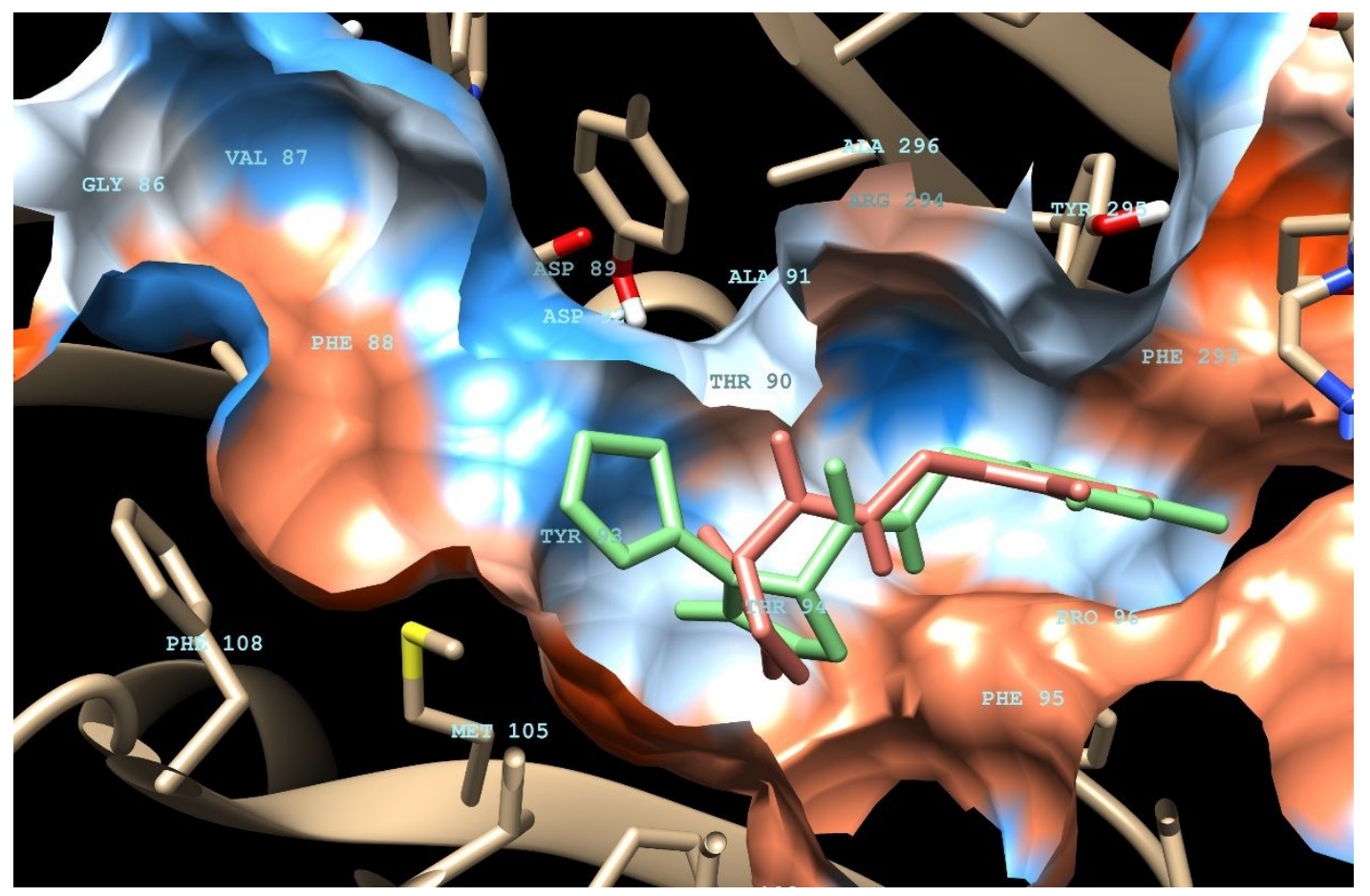

Figure S9. Docking poses predicted for 16i (light green) vs 1a (orange).

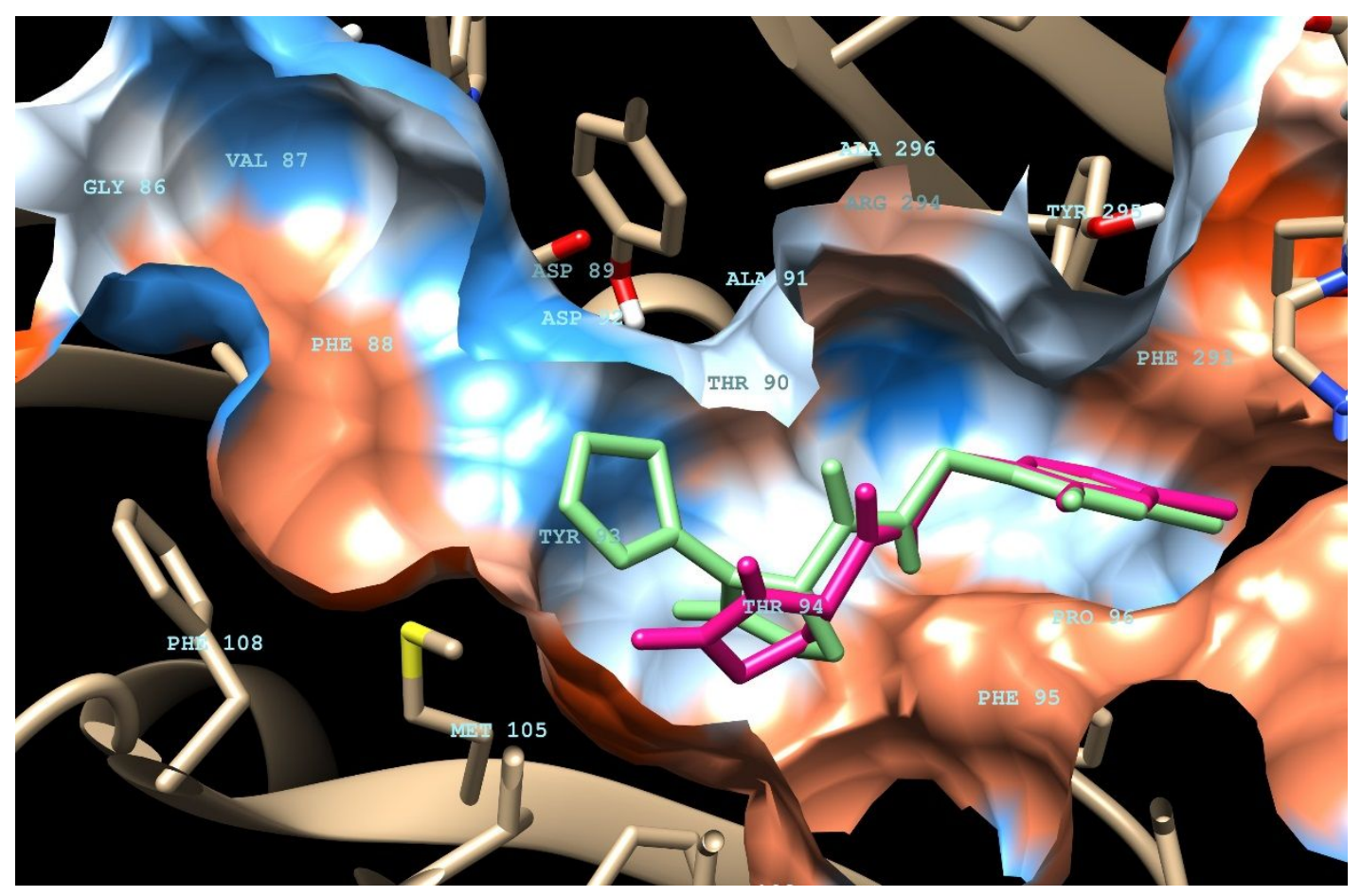

Figure S10. Docking poses predicted for 16i (light green) vs 11e (pink). 


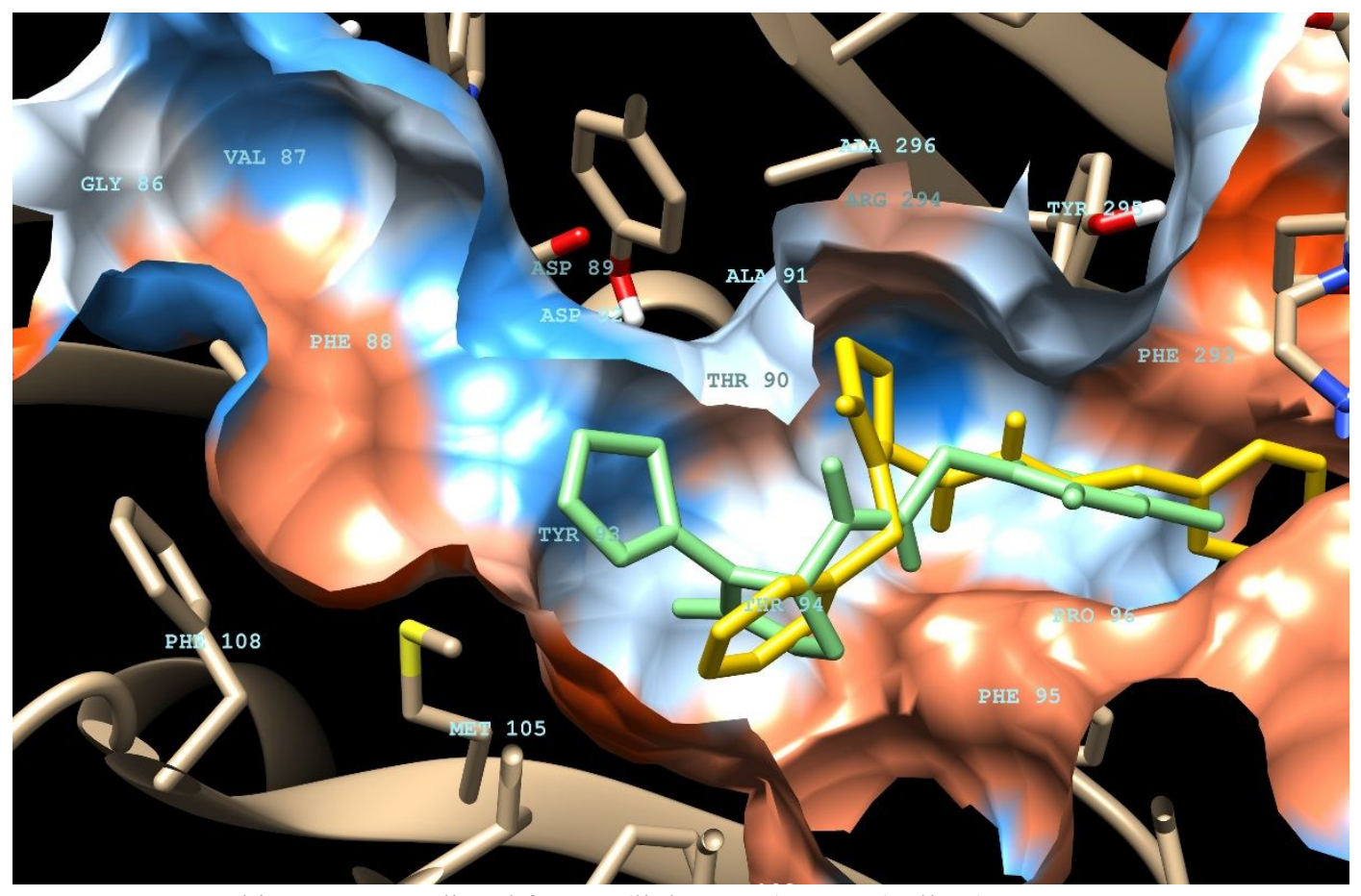

Figure S11. Docking poses predicted for 16i (light green) vs 37 (yellow).

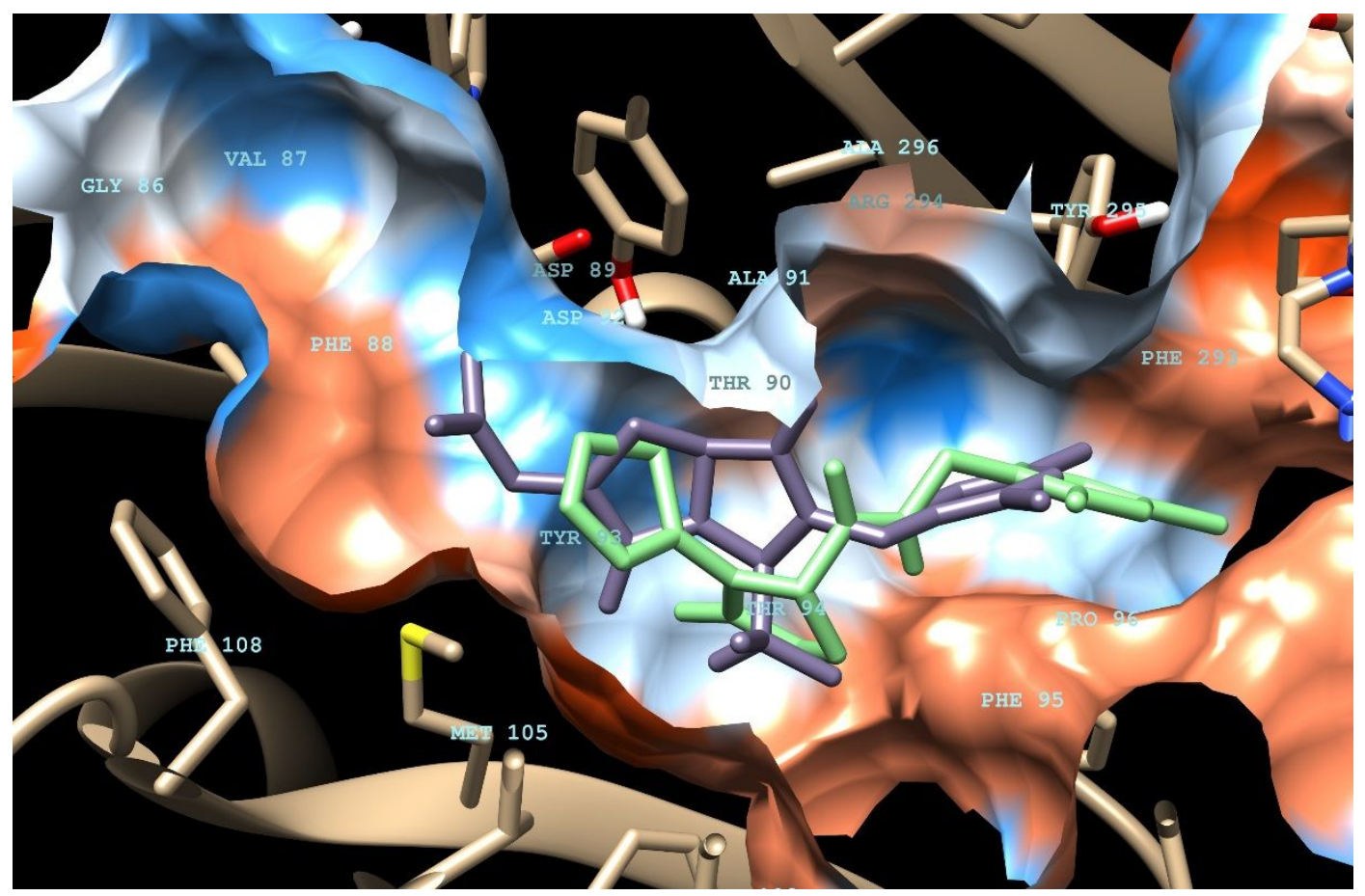

Figure S12. Docking poses predicted for 16i (light green) vs $50 \mathrm{~g} 1^{\text {st }}$ best fitting pose (grey). 


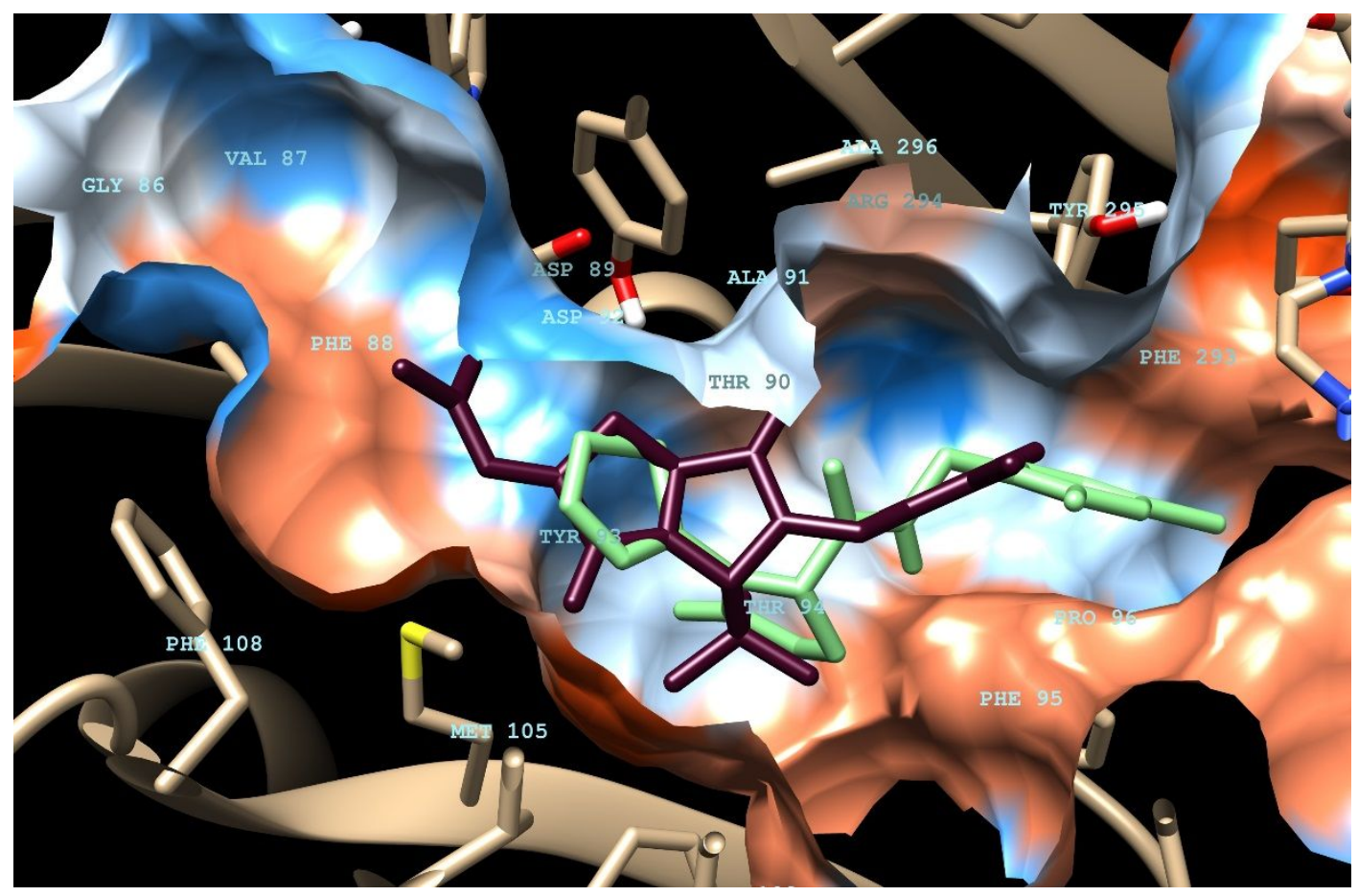

Figure S13. Docking poses predicted for $\mathbf{1 6}$ (light green) vs $\mathbf{5 0 g} 2^{\text {nd }}$ best fitting pose (dark red). 


\section{Metabolization of compound 16i:}

Materials and methods ${ }^{29}$

In vitro incubation with human liver microsomes. Briefly, once thawed, HLMs ( $2 \mathrm{mg}$ protein $/ \mathrm{mL}$ for a final volume of $100 \mu \mathrm{L}$ ) were pre-activated by alamethicin on ice in an intermediate volume of $50 \mu \mathrm{L}$ in $0.1 \mathrm{M}$ Tris-HCl- $\mathrm{MgCl}_{2}(10 \mathrm{mM} \mathrm{MgCl} 2$ and $100 \mathrm{mM}$ Tris- $\mathrm{HCl}$ solution $)$ at $\mathrm{pH}$ 7.4. This mixture was added to a 50 $\mu \mathrm{L}$-dried residue of a methanol-DMSO (9/1) solution of compound $16 \mathrm{i}$ at a concentration of $0.2 \mathrm{mg} / \mathrm{mL}$. Fifty $\mu \mathrm{L}$ of a cofactor mixture (5 mM UDPGA, $1.3 \mathrm{mM}$ NADPH, $3.3 \mathrm{mM} \mathrm{G6P}$ and $0.5 \mathrm{U} / \mathrm{mL}$ G6PD) in 0.1 $\mathrm{M}$ Tris- $\mathrm{HCl}$ was then added. The enzymatic reaction was performed at $37^{\circ} \mathrm{C}$ for $60 \mathrm{~min}$, and stopped by the addition of $100 \mu \mathrm{L}$ of methanol-containing methyl-clonazepam at $1.25 \mathrm{mg} / \mathrm{L}$ and $\beta$-OH-ethyltheophyllin at $16 \mathrm{mg} / \mathrm{L}$, used as internal standards for the chromatographic analysis. Samples were centrifuged at $4{ }^{\circ} \mathrm{C}$ for $14 \mathrm{~min}$ at $32000 \mathrm{~g}$ and supernatants were diluted in $400 \mu \mathrm{L}$ of 3\% 5-sulfosalicylic acid before injection of $75 \mu \mathrm{L}$ into the chromatographic system.

Liquid chromatography with quadrupole-time of flight mass spectrometry detection. The Waters ultraperformance liquid chromatography system consisted of two binary solvent manager LC pumps, a sample manager autosampler and a column manager oven Acquity ${ }^{\mathrm{TM}}$. Mass spectrometry data were acquired using an XEVO G2-XS QTOF (Waters, Milford, MA, USA) instrument controlled with MassLynx ${ }^{\mathrm{TM}} 4.1$ software. For mass accuracy, a LockSpray ${ }^{\mathrm{TM}}$ interface was used with a Leucine Enkephalin $\left([\mathrm{M}+\mathrm{H}]^{+} \mathrm{m} / z\right.$ $556.2771)$ solution $(200 \mathrm{pg} / \mu \mathrm{L})$ infused at $10 \mu \mathrm{L} / \mathrm{min}$ as the lock mass, providing exact mass measurement within 1 ppm RMS mass accuracy.

Extractions of the sample ( $75 \mu \mathrm{L}$ of diluted supernatants) was performed using an OASIS HLB on-line column $(30 \times 2.1 \mathrm{~mm}, 20 \mu \mathrm{m})$ (Waters, Milford, MA, USA). The mobile phases consisted in $0.2 \%$ ammonia solution (A1) and $100 \%$ methanol (B1) and the elution profile was as follows: $0.5 \mathrm{~min}, 0 \% \mathrm{~B} 1$, flow rate $2.0 \mathrm{~mL} / \mathrm{min} ; 1.0 \mathrm{~min}, 0 \% \mathrm{~B} 1$, flow rate $0.2 \mathrm{~mL} / \mathrm{min} ; 15 \mathrm{~min}, 100 \% \mathrm{~B}$, flow rate $0.2 \mathrm{~mL} / \mathrm{min} ; 30 \mathrm{~min}, 100 \%$ B1, flow rate $2.0 \mathrm{~mL} / \mathrm{min}$. The chromatographic separation was performed using an Acquity HSS C18 column $(150 \times 2 \mathrm{~mm}, 1.8 \mu \mathrm{m})$ (Waters, Milford, MA, USA) in an oven temperature of $50{ }^{\circ} \mathrm{C}$, and mobile phases including ammonium formate buffer $5 \mathrm{mM}$, pH 3 (A2) and acetonitrile in 1\% formic acid (B2); flow rate of $0.4 \mathrm{~mL} / \mathrm{min}$ was used. Initial concentration of mobile phase B2 (3\%) held until $0.5 \mathrm{~min}$, increased to $40 \%$ at $25 \mathrm{~min}$, then to $100 \%$ at $26 \mathrm{~min}$, and finally held until $30 \mathrm{~min}$.

For detection, mass spectrometric conditions were as follows: positive electrospray ionization interface $(\mathrm{ESI}+), 20 \mathrm{~V}$ as ion spray voltage, source temperature at $140{ }^{\circ} \mathrm{C}$ and desolvatation temperature at $500{ }^{\circ} \mathrm{C}$ with a desolvation gas flow rate of $900 \mathrm{~L} / \mathrm{h}$, nitrogen as desolvation gas and argon as collision gas. Conditions for the time of flight mass spectrometer (TOF MS ) scanmodewere as follows: scan range 100$1000 \mathrm{~m} / \mathrm{z}$ for function 1 and $50-1000 \mathrm{~m} / \mathrm{z}$, with a collision energy ramp from 10-40 eV, for function 2 .

Data processing and evaluation of compound 16i metabolites were performed using the Metabolynx ${ }^{\mathrm{TM}} 4.1$ software (Waters, Milford, MA, USA). Theoretically possible biotransformations of compound 16i were simulated, including possible hydroxylation, $N$-dealkylation, deamination and glucuronidation transformations. The automated metabolite profiling process was completed using the fragmentation interpretation software tool MassFragment ${ }^{\mathrm{TM}} 4.1$ (Waters, Milford, MA, USA), to allow software driven assignment of metabolite structures from fragmentation patterns.

As previously reported, a control sample containing propafenone was analyzed using the incubation and chromatographic conditions as for the metizolam in order to assess the efficacy of the enzymatic system. The efficacy was considered to be correct if the production of both main CYP- and UGT-derived metabolites of propafenone (i.e., hydroxypropafenone and hydroxypropafenone glucuronide) was observed.

$\underline{\text { Results }}$

Figure 14 shows UPLC spectrograms of pure compound 16i (Figure 14.A), pure compound 10e (Figure 14.B) and supernatant of the metabolization assay of $\mathbf{1 6} \mathbf{i}$, where only detected mass of $158.9768 \pm 0.0002$ g. $\mathrm{mol}^{-1}$ (Figure 14.C) corresponding to common mass fragmentation of 10e (red pic, retention time $=6.43$ 
min, mass spectrum is shown in figure 14.D) and 16i (purple pic, retention time $=9.68 \mathrm{~min}$, mass spectrum is shown in figure 14.E). The black pic is an artefact observed in other assays belonging to non-related studies and associated with the protocol, moreover no fragments of the mass spectrum of this pic correspond to a potential fragment of $\mathbf{1 6} \mathbf{i}$ or to a potential fragment of a metabolite of $\mathbf{1 6} \mathbf{i}$. No other significant pic, except pics associated with the protocol and commonly observed, was seen in the chromatogram of the supernatants for complete mass spectrum (not shown). These results suggest that the main metabolite of $N$ heteroaryl-PGAm 16i is the $N H$-free lactam 10e.
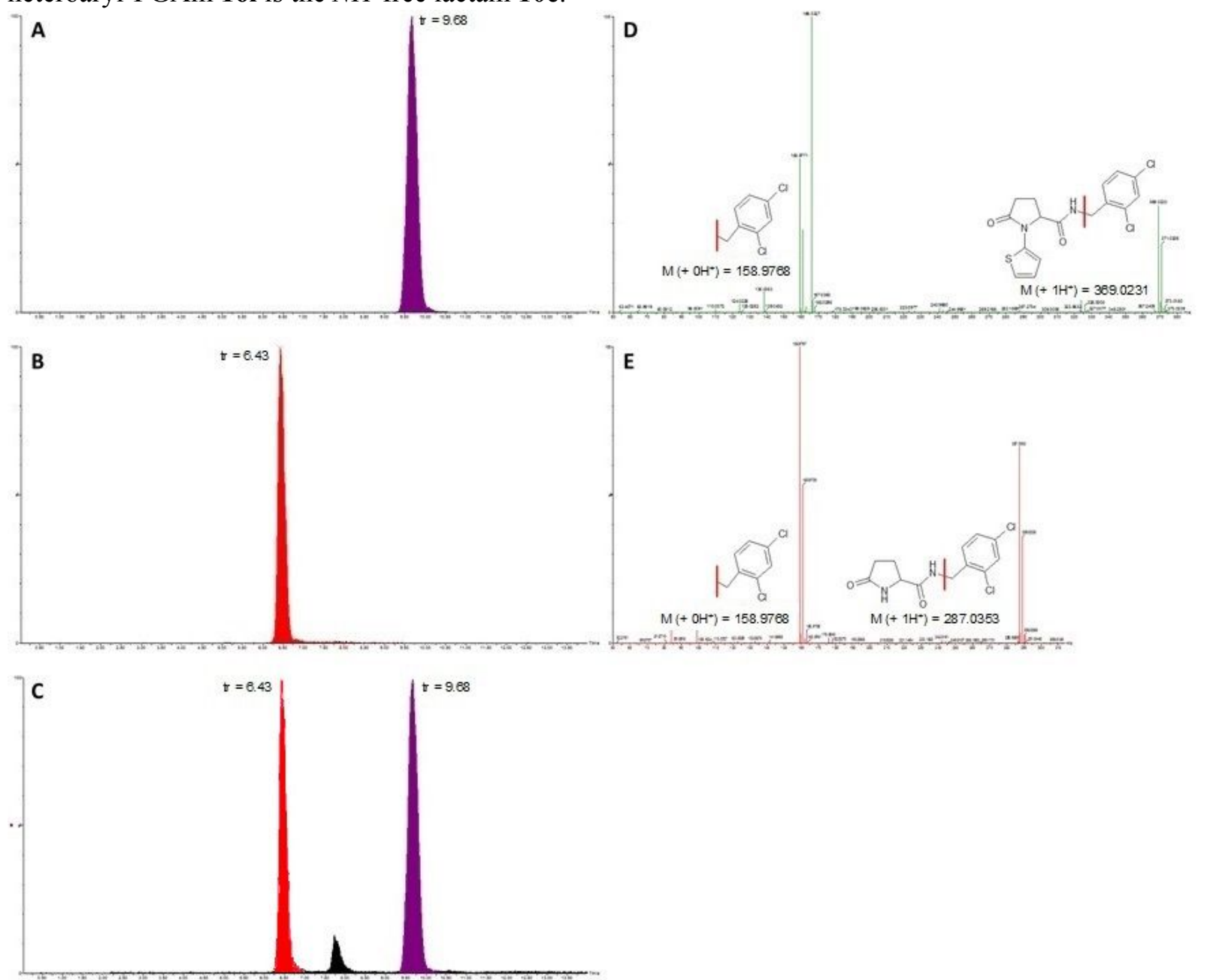

Figure S14. Metabolization of compound 16i by human hepatic microsomes. A: UPLC spectrogram of pure compound 16i; B: UPLC spectrogram of pure compound 10e; C: UPLC spectrogram of supernatant of the metabolization assay of 16i, where only detected mass of $158.9768 \pm 0.0002 \mathrm{~g}^{\mathrm{mol}} \mathrm{mol}^{-1}$ is shown; D: compound 10e (red pics) mass spectrum and structures of main fragments; E: compound 16i (purple pics) mass spectrum and structures of main fragments. 


\section{Platelet aggregation assay.}

Materials and methods

Preparation of washed platelets. Whole blood was collected from HDs. Platelets were isolated by differential centrifugation at increasing acceleration and then washed in Hank's balanced salt solution (HBSS). ${ }^{30}$

Platelet aggregation assay. Washed human platelets $(107 \mathrm{cells} / \mathrm{ml})$ were preincubated with Bz-ATP and Lipopolysaccharides (LPS) $(10 \mu \mathrm{g} / \mathrm{mL})$ and with P2X7 receptor antagonists $\mathbf{1 b}$ and $\mathbf{1 6} \mathbf{i}$ at a concentration of $10^{-5}$ or $10^{-6}$ for $10 \mathrm{~min}$ in HBSS solution and then treated with either thrombin $(0.2 \mathrm{U} / \mathrm{ml})$, to induce platelet aggregation. Platelet aggregation was recorded continuously after addition of the agonist for 8 min using an aggregometer (LABITEC APACT $\left.{ }^{\circledR} 4004\right)$.

$\underline{\text { Results }}$

No significant effect was observed, indicating the probable absence of interaction of studied compounds (Mol1 = compound 1b, Mol2 = compound 16i) with P2RX1 and influence on the platelets aggregation.

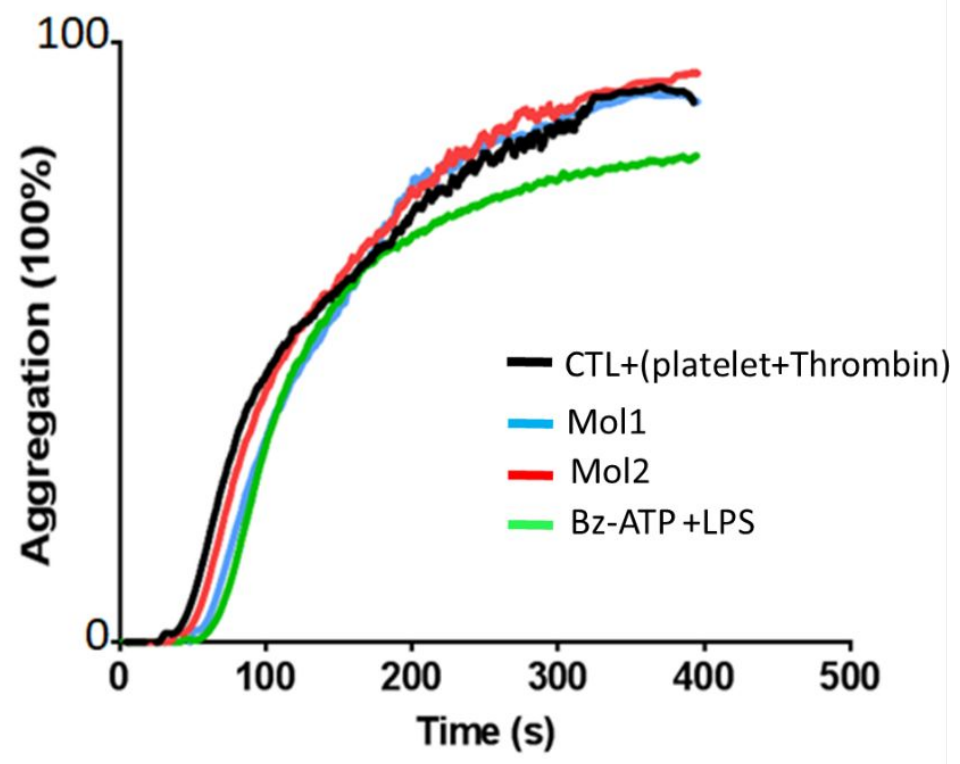

Figure S15. Platelet aggregation after exposure to compounds $\mathbf{1 b}$ (Mol1) and 16i (Mol2). 


\section{Cell proliferation assay:}

The cell proliferation assay was realized with 65 selected compounds on a panel of 60 human cancer cell lines at the National Cancer Institute, Germantown, MD, USA and was conducted using a 48h exposure using the sulfurhodamine B assay. One dose mean graphics are given bellow. 


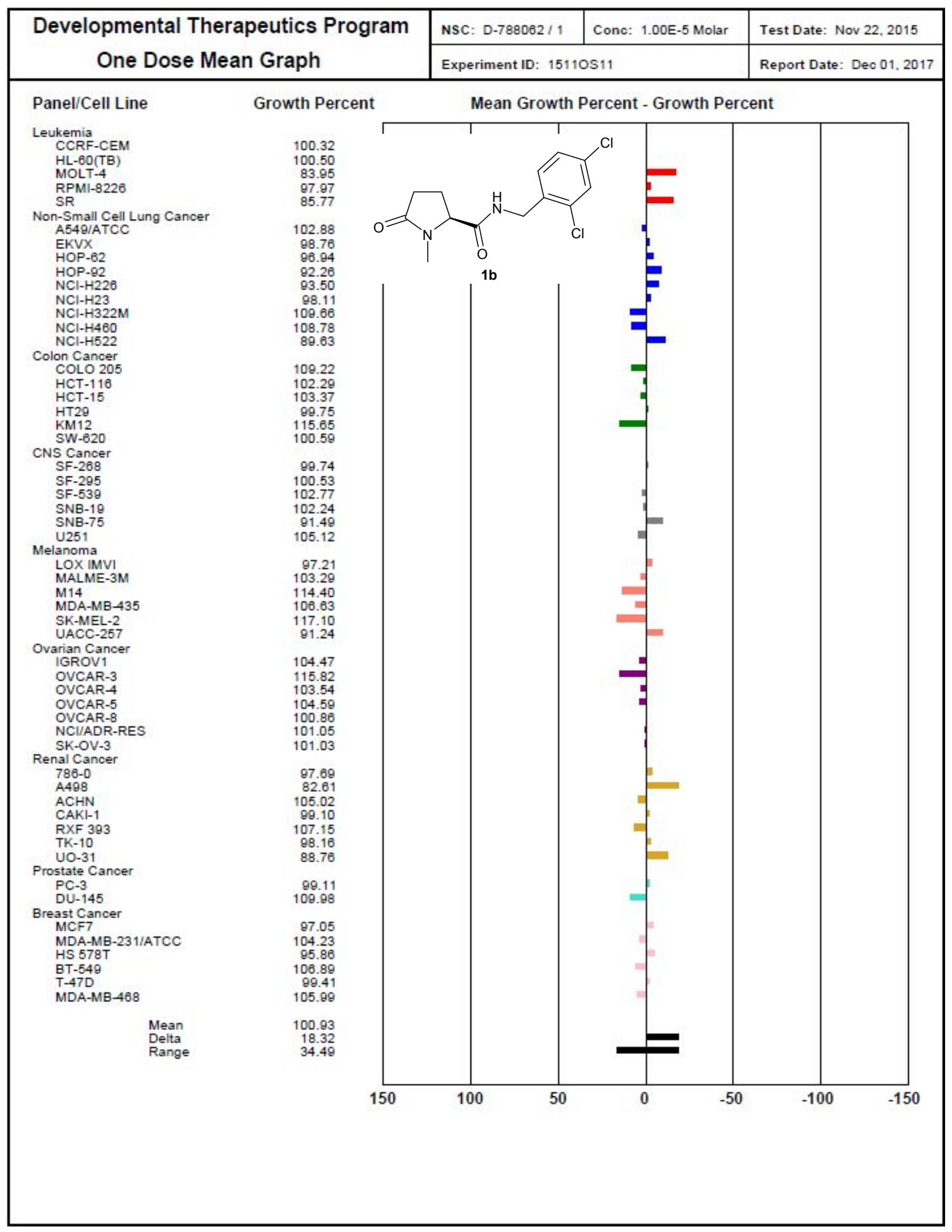




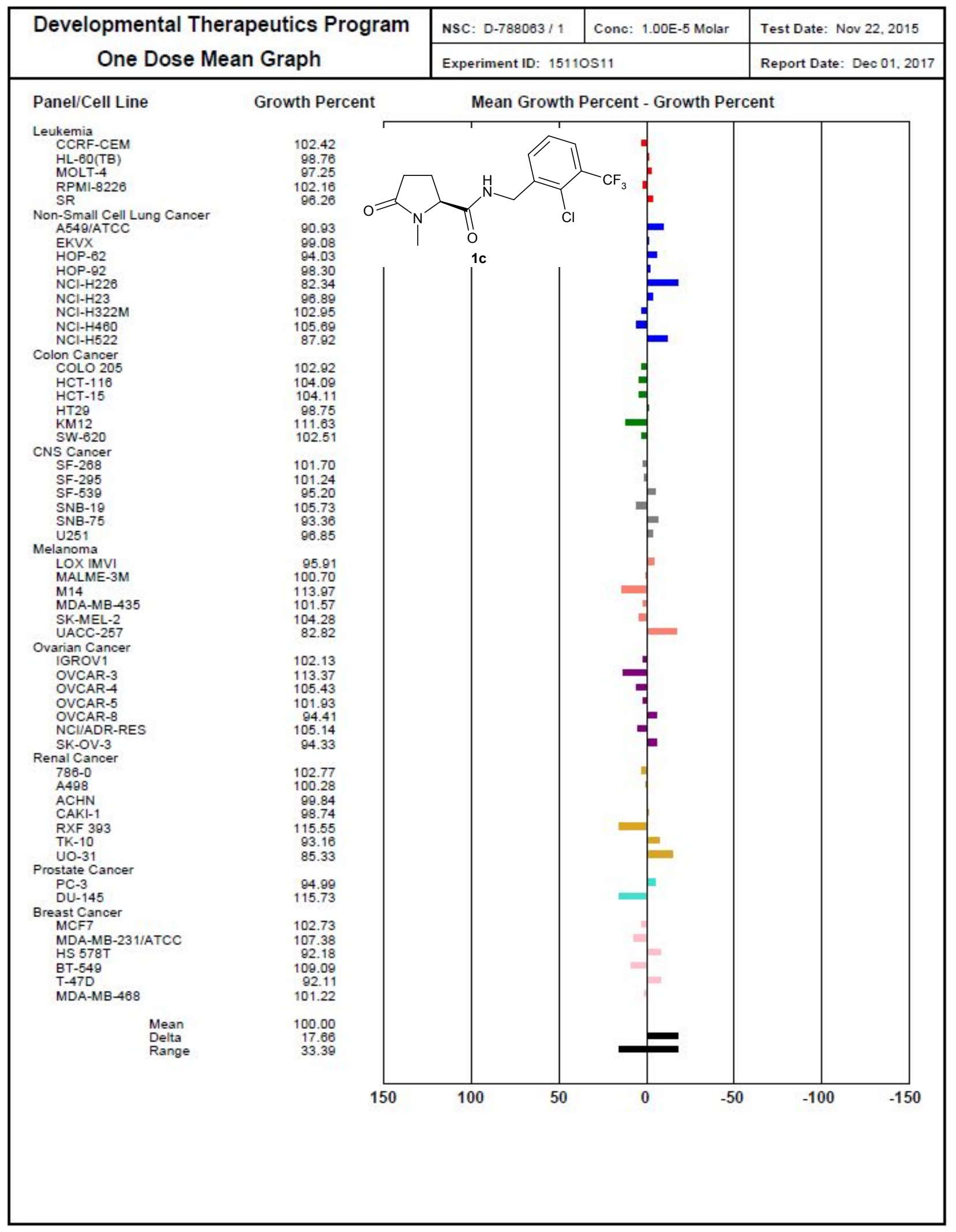




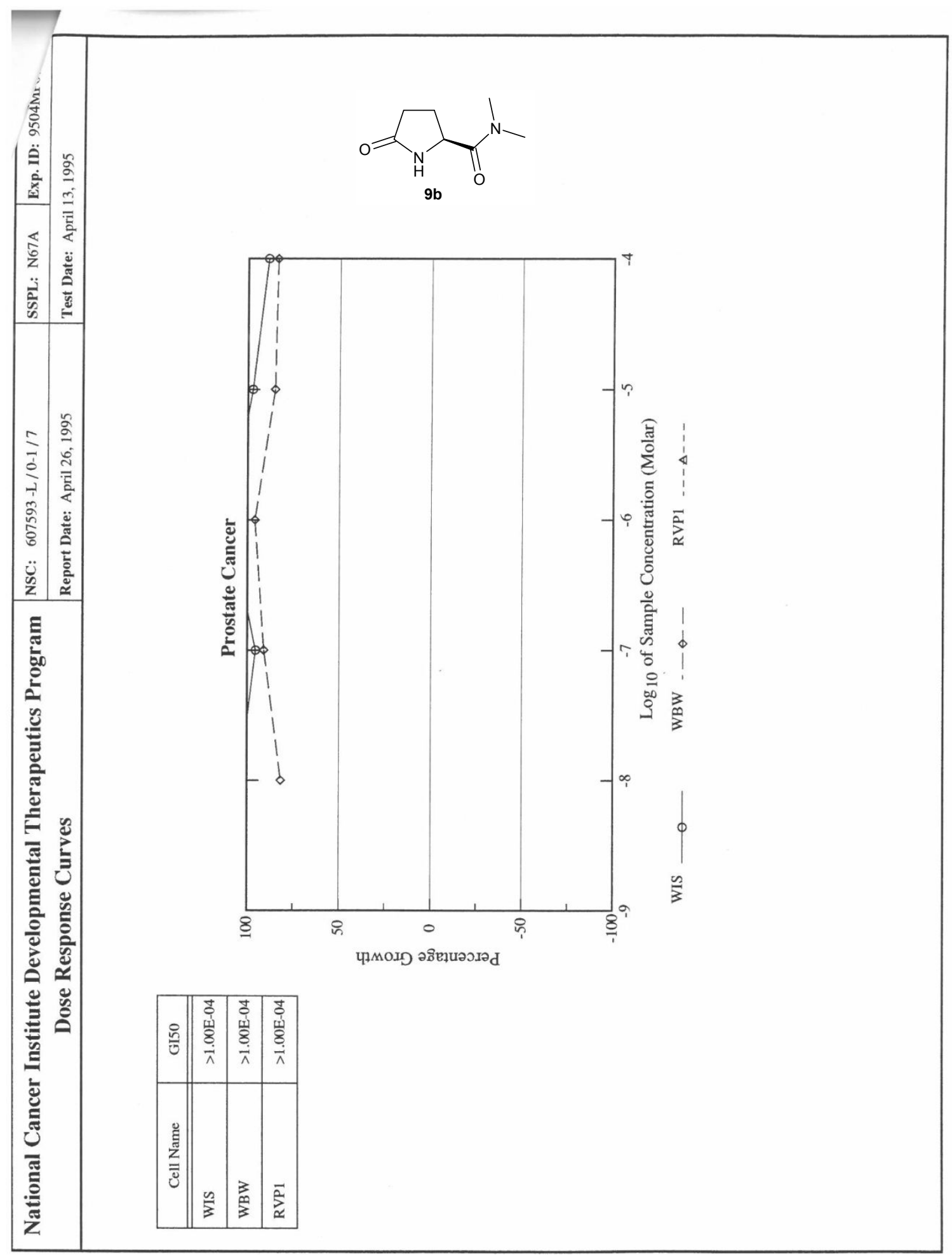

$7 \mathrm{~g}$ 


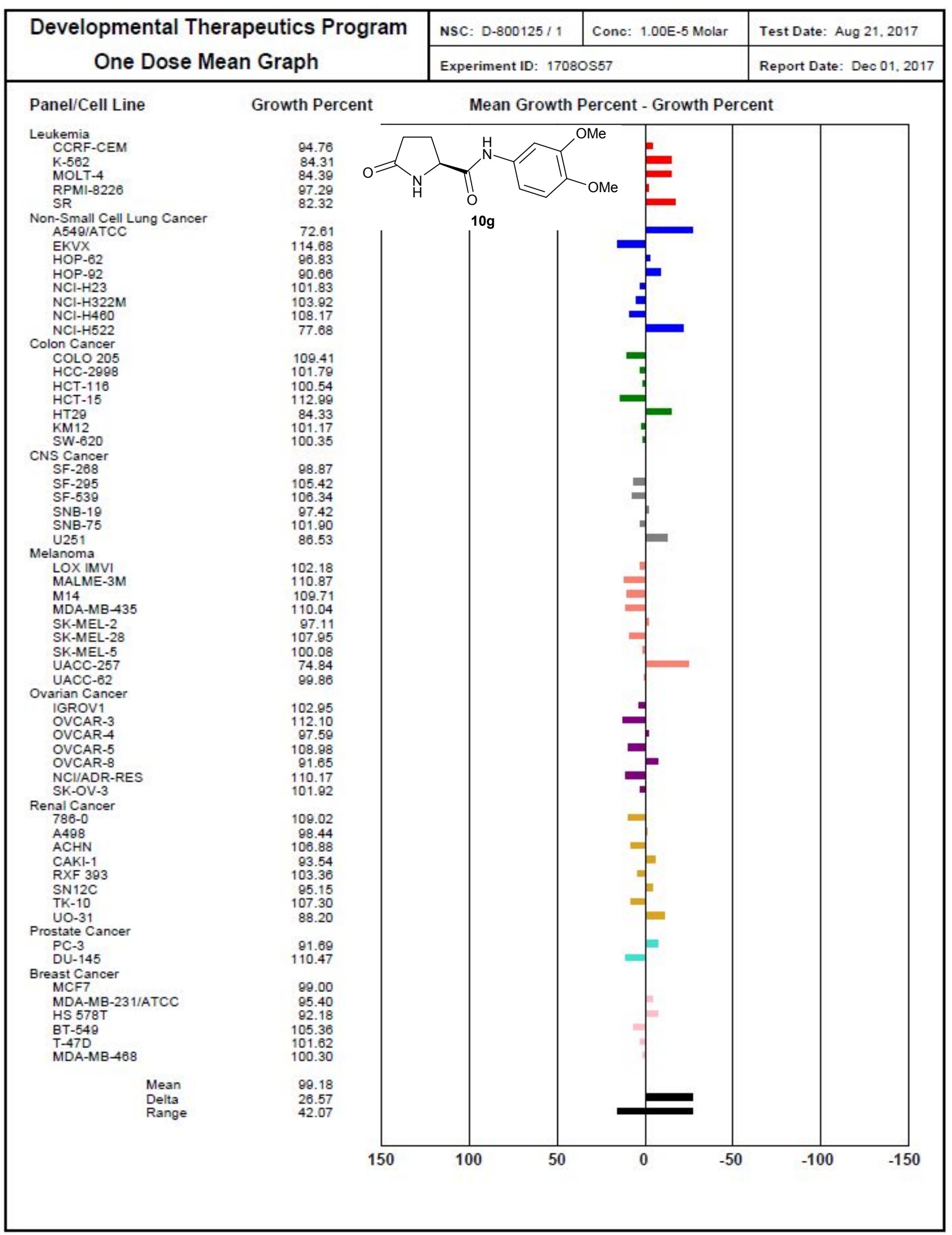




\begin{tabular}{|c|c|c|c|c|c|}
\hline \multirow{2}{*}{\multicolumn{2}{|c|}{$\begin{array}{c}\text { Developmental Therapeutics Program } \\
\text { One Dose Mean Graph }\end{array}$}} & NSC: D-798011/1 & Conc: $1.00 E-5$ Molar & \multirow{2}{*}{\multicolumn{2}{|c|}{$\begin{array}{l}\text { Test Date: May 08, } 2017 \\
\text { Report Date: Dec 01, } 2017\end{array}$}} \\
\hline & & \multicolumn{2}{|c|}{ Experiment ID: 17050500} & & \\
\hline \multicolumn{2}{|c|}{ Panel/Cell Line $\quad$ Growth Percent } & Mean Growth & Percent - Growth Per & ent & \\
\hline \multicolumn{6}{|c|}{$\begin{array}{l}\text { Leukemia } \\
\text { CCRF-CEM }\end{array}$} \\
\hline $\begin{array}{l}\mathrm{HL}-60(\mathrm{~TB}) \\
\mathrm{K}-562\end{array}$ & $\begin{array}{l}11.08 \\
127.99 \\
105.62\end{array}$ & & & & \\
\hline $\begin{array}{l}\text { MOLT - } \\
\text { MPLML-8226 } \\
\text { RPM }\end{array}$ & $\begin{array}{r}98.18 \\
115.54\end{array}$ & & & & \\
\hline SR & $\begin{array}{r}115.54 \\
94.30\end{array}$ & & & & \\
\hline \multicolumn{6}{|l|}{$\begin{array}{l}\text { Non-Small Cell Lung Cancer } \\
\text { A549/ATCC }\end{array}$} \\
\hline \multirow{2}{*}{\multicolumn{6}{|c|}{$\begin{array}{l}\text { A554/ATCC } \\
\text { EKVX } \\
\text { HOP-62 } \\
\text { HOP-92 }\end{array}$}} \\
\hline & $\begin{array}{r}112.24 \\
93.15\end{array}$ & & & & \\
\hline \multicolumn{6}{|l|}{$\begin{array}{l}\mathrm{NCl}-\mathrm{H} 228 \\
\mathrm{NC}-\mathrm{H} 23\end{array}$} \\
\hline \multicolumn{6}{|l|}{$\begin{array}{l}\mathrm{NCl}-\mathrm{H} 322 \mathrm{M} \\
\mathrm{NCl}-\mathrm{H} 460\end{array}$} \\
\hline \multicolumn{6}{|l|}{$\begin{aligned} \mathrm{NCCl}-\mathrm{H} 252 \\
\text { Colon Cancer }\end{aligned}$} \\
\hline \multicolumn{6}{|l|}{$\begin{array}{l}\text { Colon Cancer } \\
\text { COLO } 205 \\
\text { HCC-2998 }\end{array}$} \\
\hline $\begin{array}{l}\text { HCC-2998 } \\
\text { HCT-116 }\end{array}$ & $\begin{array}{l}96.53 \\
99.39\end{array}$ & & & & \\
\hline \multicolumn{6}{|l|}{$\begin{array}{l}\text { HCT-116 } \\
\text { HCT-15 } \\
\text { HT⿰冫中 }\end{array}$} \\
\hline \multicolumn{6}{|l|}{$\begin{array}{l}\text { K128 } \\
\text { KM12 } \\
\text { SW-620 }\end{array}$} \\
\hline \multicolumn{6}{|l|}{ CNS Cancer } \\
\hline \multirow{2}{*}{\multicolumn{6}{|c|}{$\begin{array}{l}\text { SF-268 } \\
\text { SF-295 } \\
\text { SF-539 }\end{array}$}} \\
\hline \multirow{2}{*}{\multicolumn{5}{|c|}{$\begin{array}{l}\text { SN-639 } \\
\text { SNB- } 75\end{array}$}} & \\
\hline & & & & & \\
\hline \multicolumn{6}{|l|}{$\begin{array}{l}\text { U251 } \\
\text { Melanoma }\end{array}$} \\
\hline \multicolumn{6}{|l|}{$\begin{array}{l}\text { LOX IMVI } \\
\text { MALME-3M }\end{array}$} \\
\hline \multicolumn{6}{|l|}{$\begin{array}{l}\text { MALME-3M } \\
\text { M14 }\end{array}$} \\
\hline \multirow{2}{*}{\multicolumn{6}{|c|}{$\begin{array}{l}\text { SK-MEL-2 } \\
\text { SK-MEL-28 } \\
\text { SK-MEL-5 }\end{array}$}} \\
\hline & $\begin{array}{l}102.48 \\
111.54\end{array}$ & & & & \\
\hline & $\begin{array}{l}111.04 \\
11.06\end{array}$ & & & & \\
\hline \multicolumn{6}{|l|}{$\begin{array}{l}\text { UACC-62 } \\
\text { Ovarian Cancer }\end{array}$} \\
\hline \multirow{2}{*}{\multicolumn{6}{|c|}{$\begin{array}{l}\text { IGROV1 } \\
\text { OVCAR-3 } \\
\text { OVCAR-4 }\end{array}$}} \\
\hline & 114.45 & & & & \\
\hline $\begin{array}{l}\text { OVCAR-5 } \\
\text { OVCAR-8 }\end{array}$ & $\begin{array}{r}92.36 \\
118.17\end{array}$ & & & & \\
\hline $\begin{array}{l}\text { NCI/ADR-RES } \\
\text { SK-OV-3 }\end{array}$ & $\begin{array}{r}95.29 \\
124.23\end{array}$ & & & & \\
\hline Renal Cancer & 187.20 & & & & \\
\hline ACHN & $\begin{array}{l}10.12 \\
102.68\end{array}$ & & & & \\
\hline $\begin{array}{l}\text { CAKl-1 } \\
\text { RXF } 393\end{array}$ & $\begin{array}{r}100.53 \\
9.47\end{array}$ & & $=$ & & \\
\hline $\begin{array}{l}\text { SN12C } \\
\text { TK-10 }\end{array}$ & $\begin{array}{r}97.88 \\
105.58\end{array}$ & & $F$ & & \\
\hline vo-31 & $\begin{array}{r}105.58 \\
98.06\end{array}$ & & $=$ & & \\
\hline $\begin{array}{l}\text { Prostate Cancer } \\
\text { PC-3 }\end{array}$ & 105.54 & & & & \\
\hline $\begin{array}{l}\text { DU-145 } \\
\text { Breast Cancer }\end{array}$ & 107.83 & & & & \\
\hline MCF7 & $\begin{array}{l}101.23 \\
107.8\end{array}$ & & & & \\
\hline $\begin{array}{l}\text { MDA-MB-231/ATCC } \\
\text { HS 578T }\end{array}$ & $\begin{array}{l}107.18 \\
105.21\end{array}$ & & & & \\
\hline $\begin{array}{l}\mathrm{BT}-549 \\
\mathrm{~T}-47 \mathrm{D}\end{array}$ & $\begin{array}{l}105.85 \\
113.85\end{array}$ & & $=$ & & \\
\hline MDA-MB-468 & 112.81 & & 4 & & \\
\hline $\begin{array}{l}\text { Mean } \\
\text { Delta }\end{array}$ & $\begin{array}{l}104.91 \\
1828\end{array}$ & & & & \\
\hline $\begin{array}{l}\text { Delta } \\
\text { Range }\end{array}$ & $\begin{array}{l}16.26 \\
40.00\end{array}$ & & & & \\
\hline & 150 & 100 & 0 & -100 & -150 \\
\hline
\end{tabular}




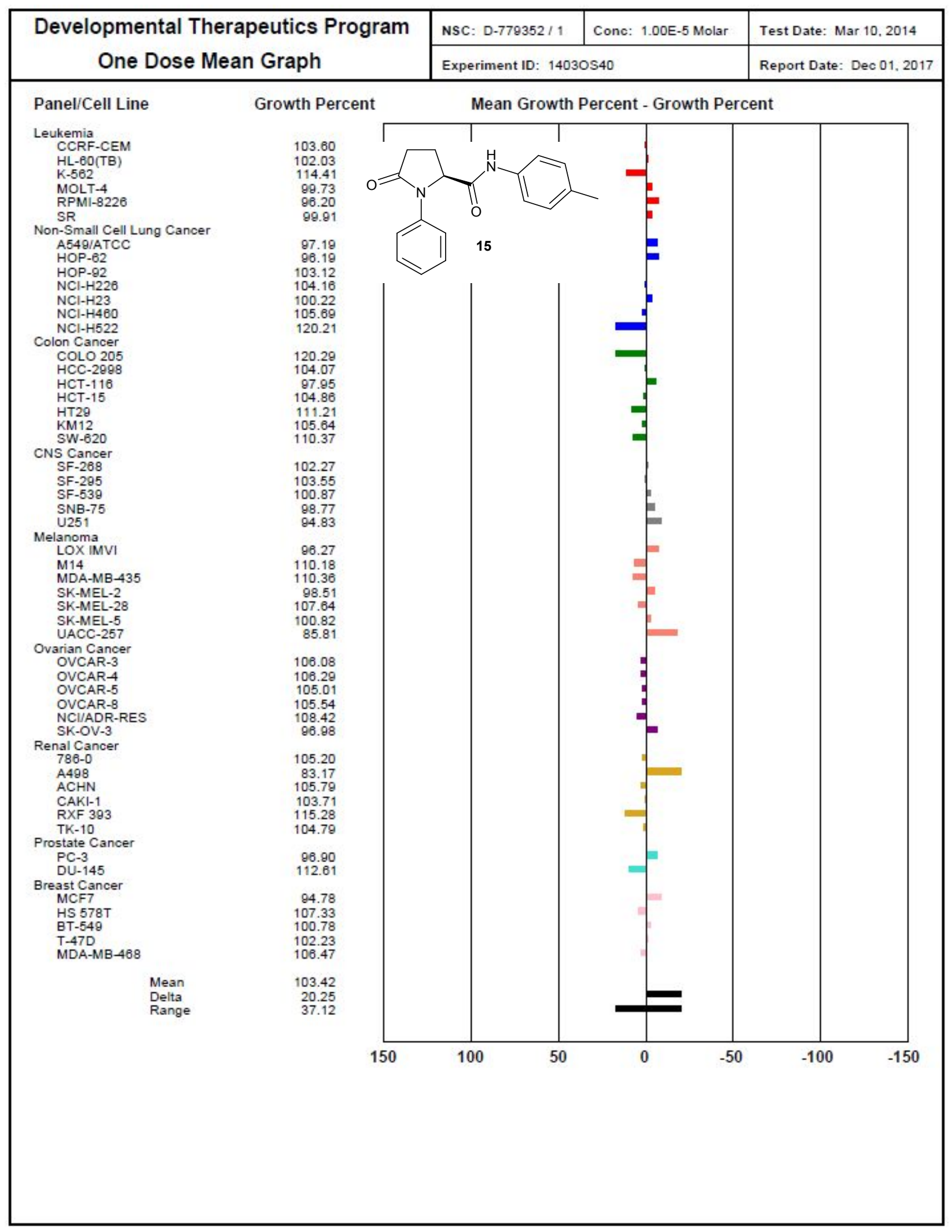




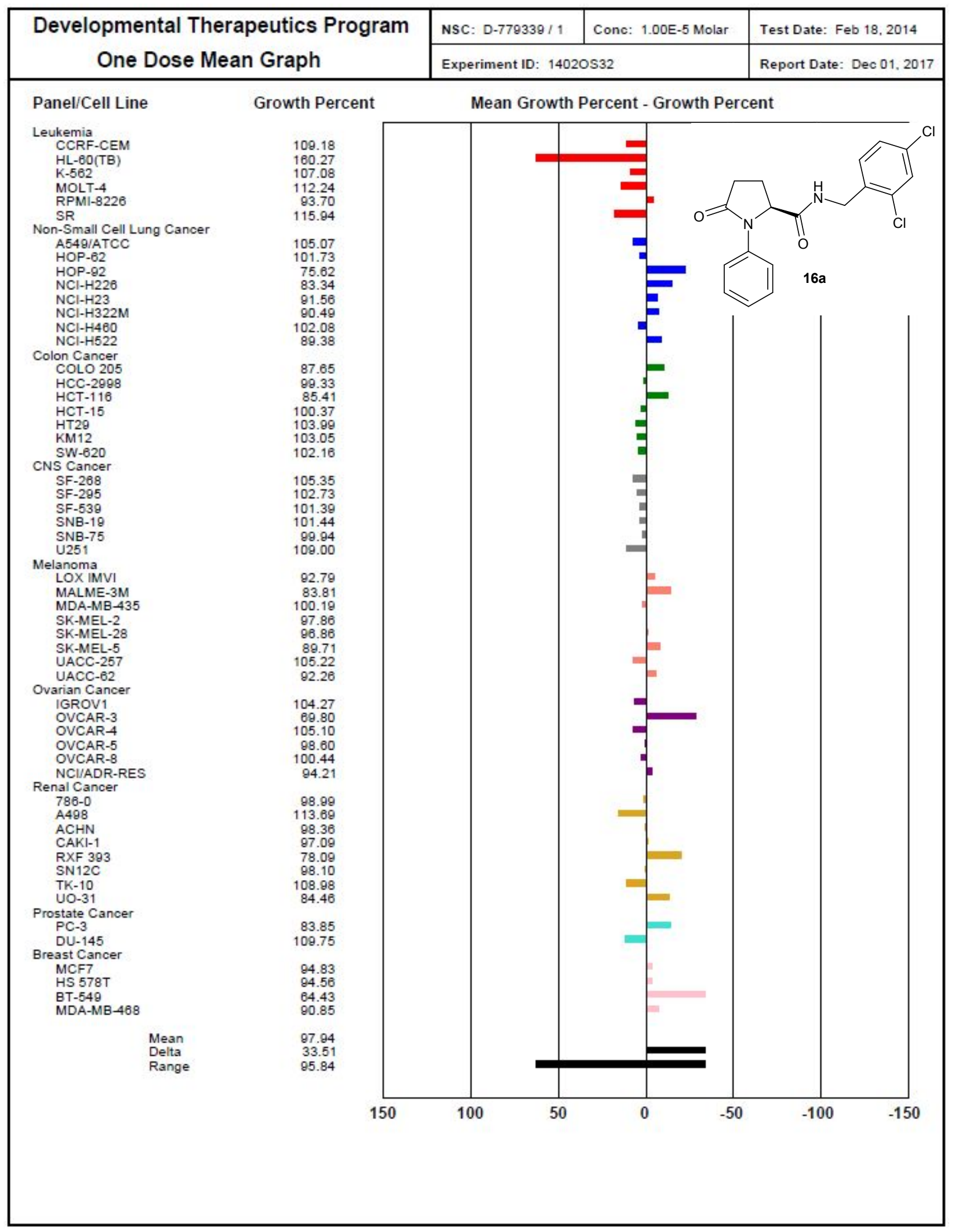




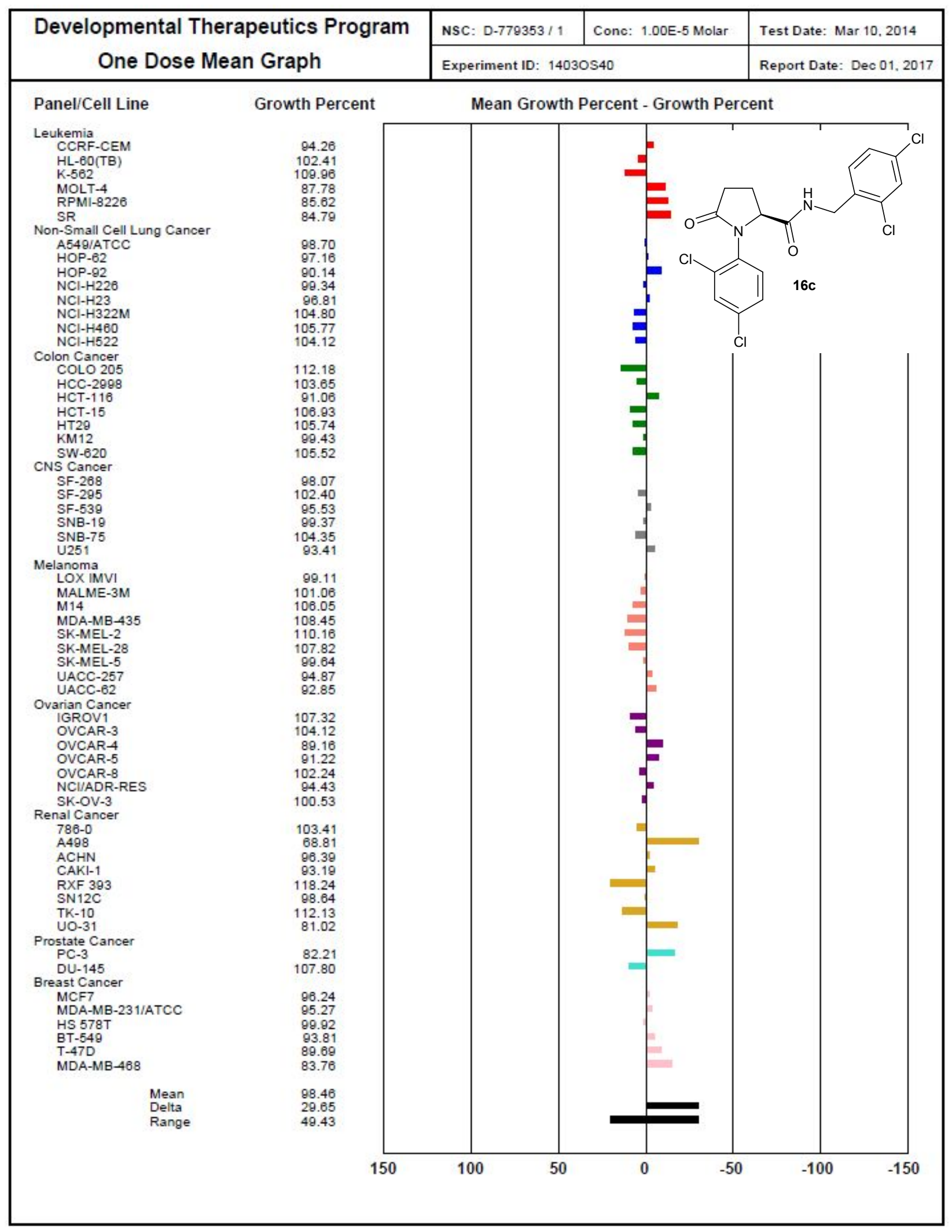




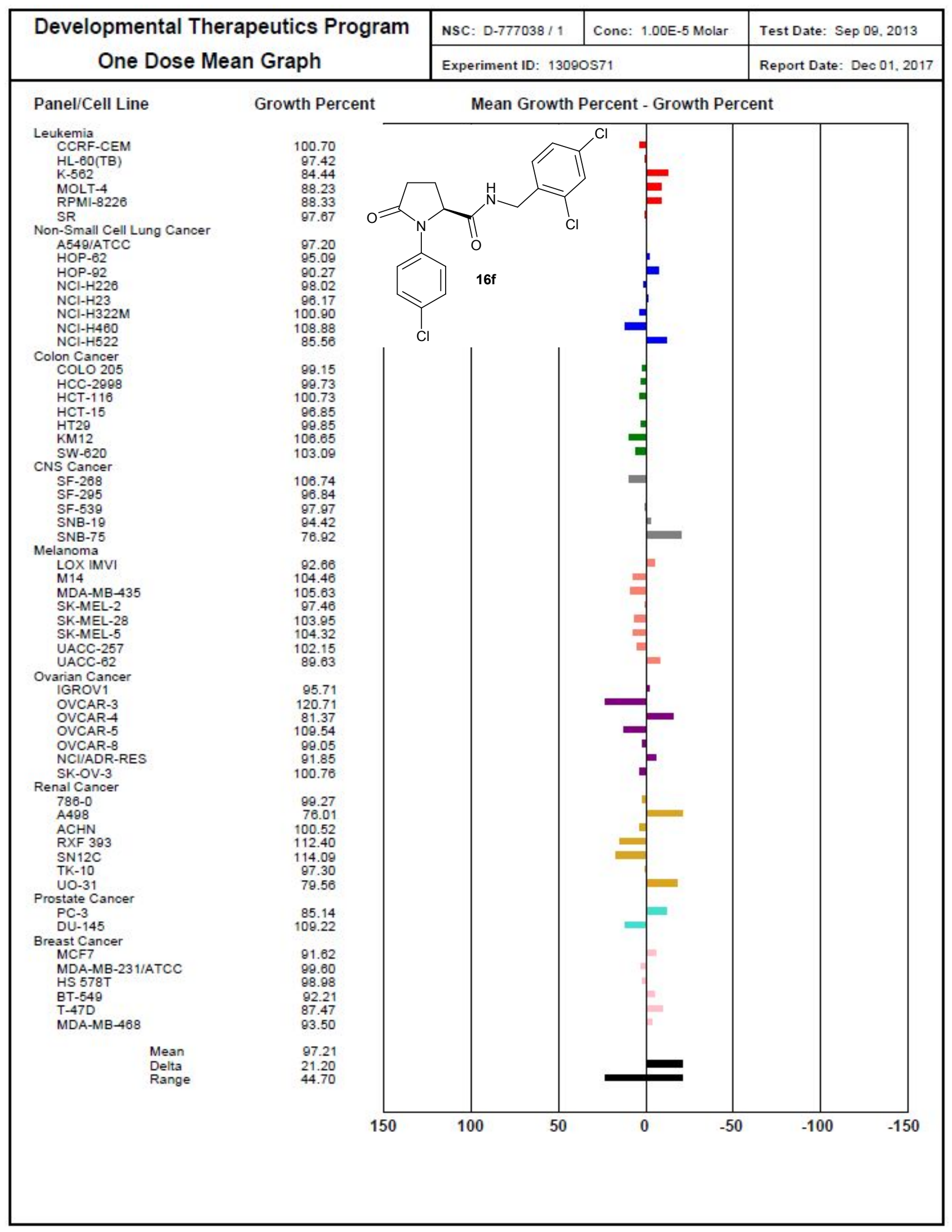




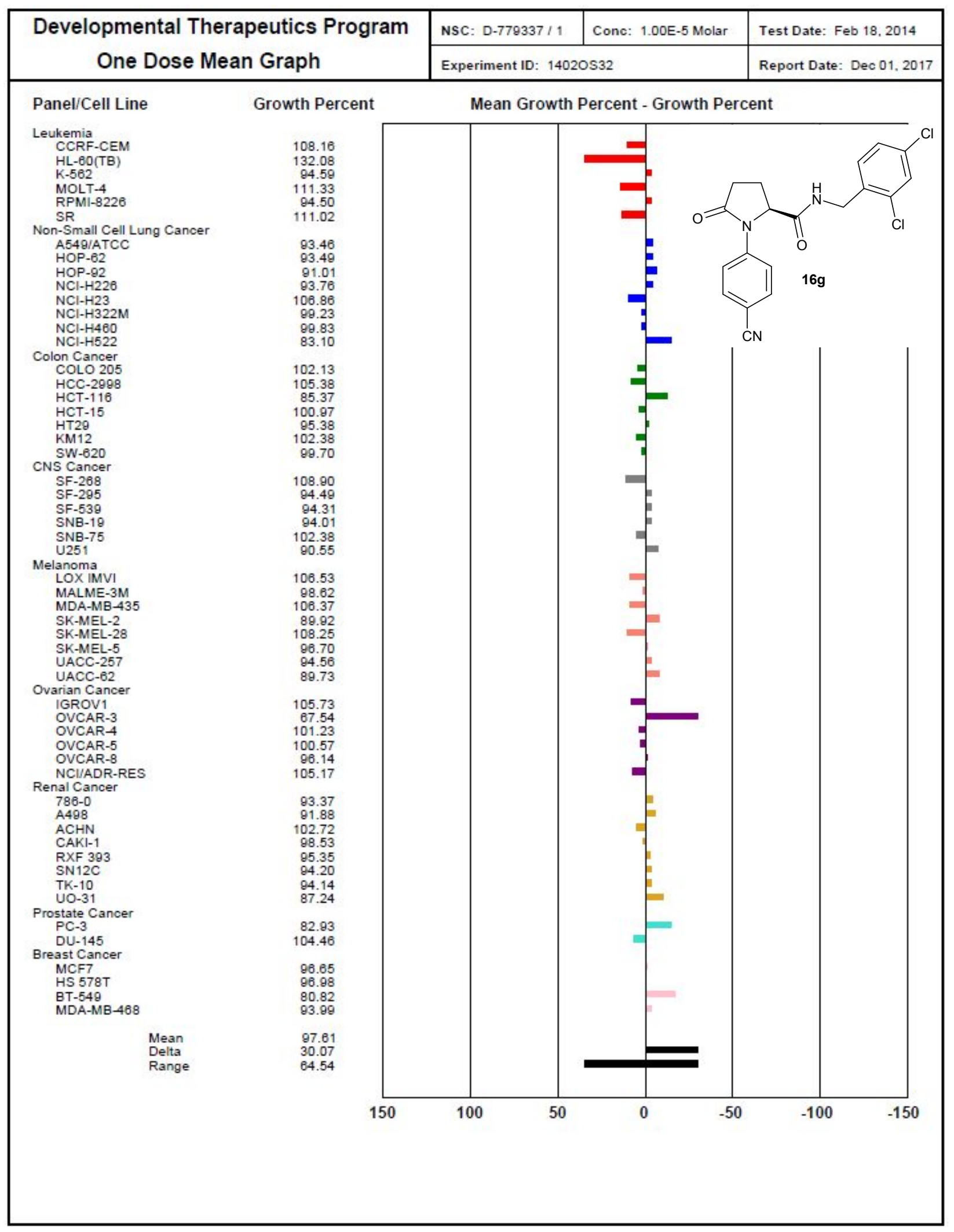




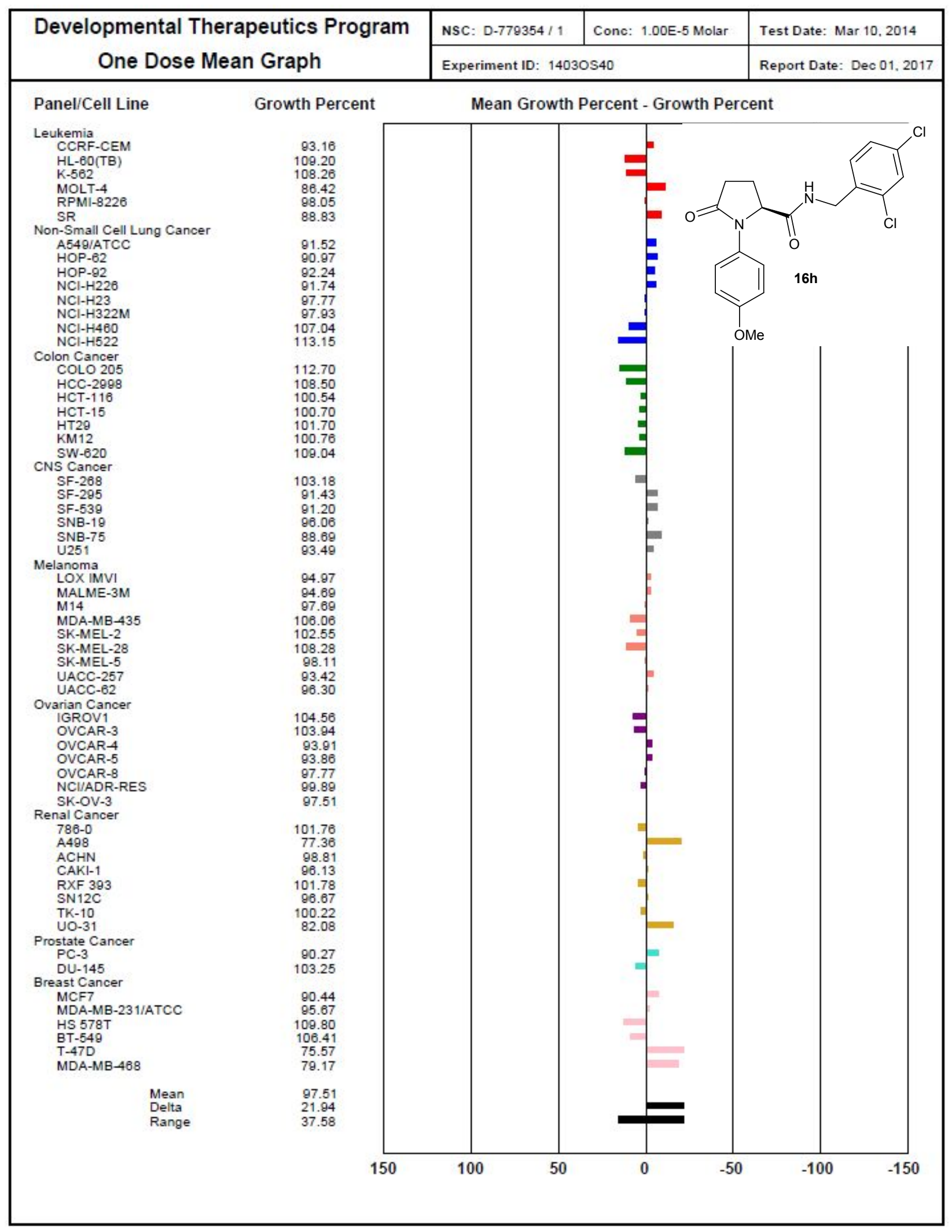




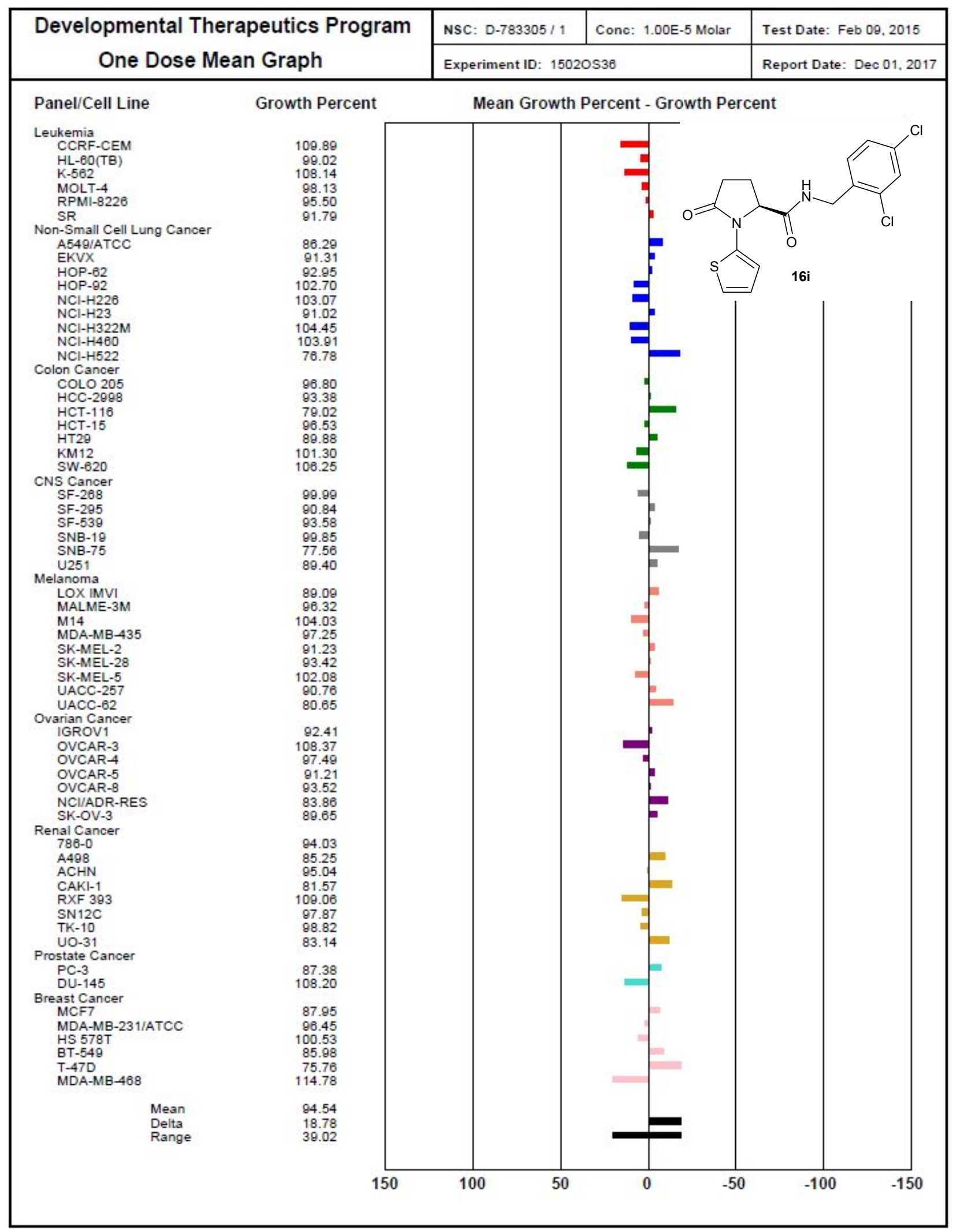




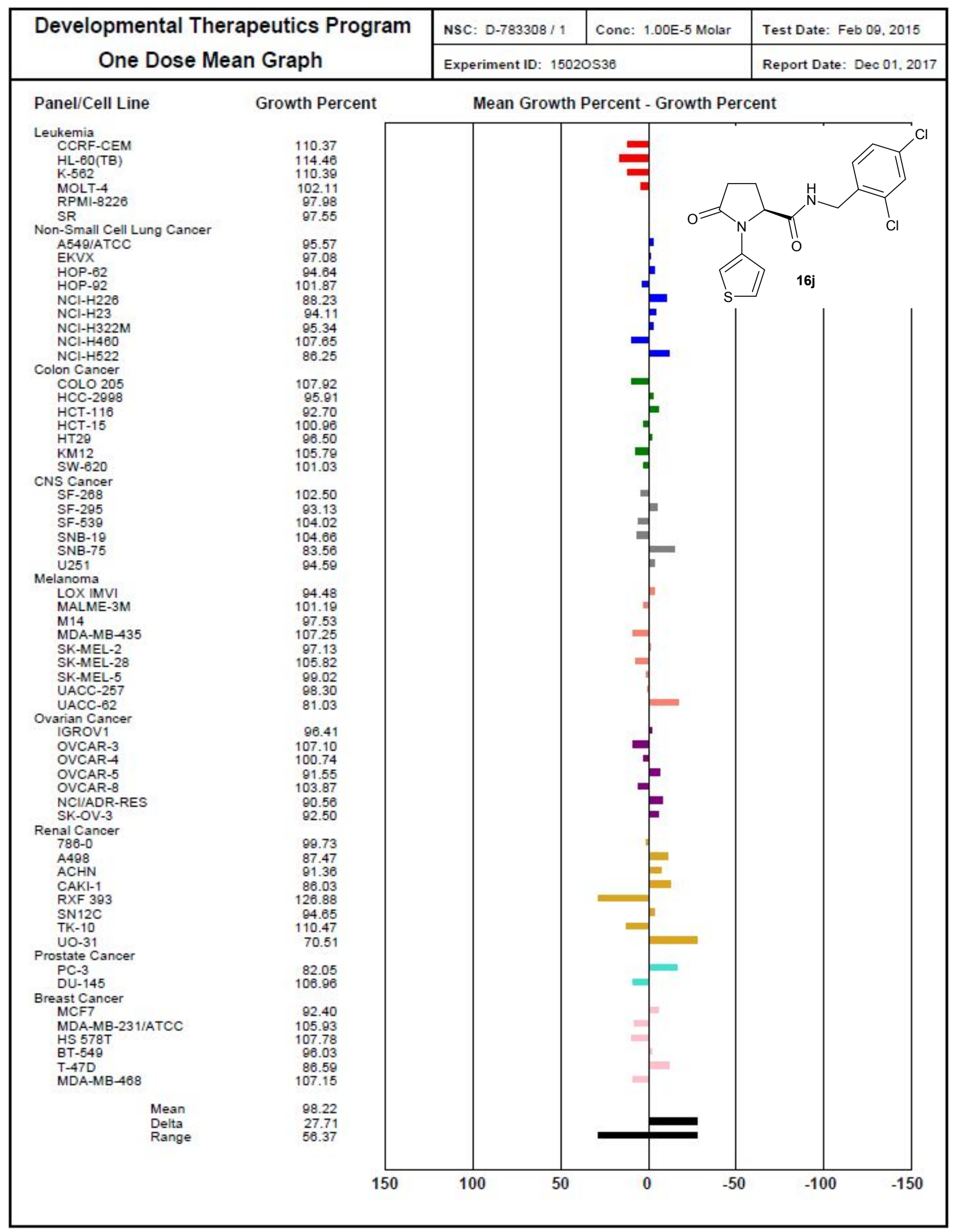




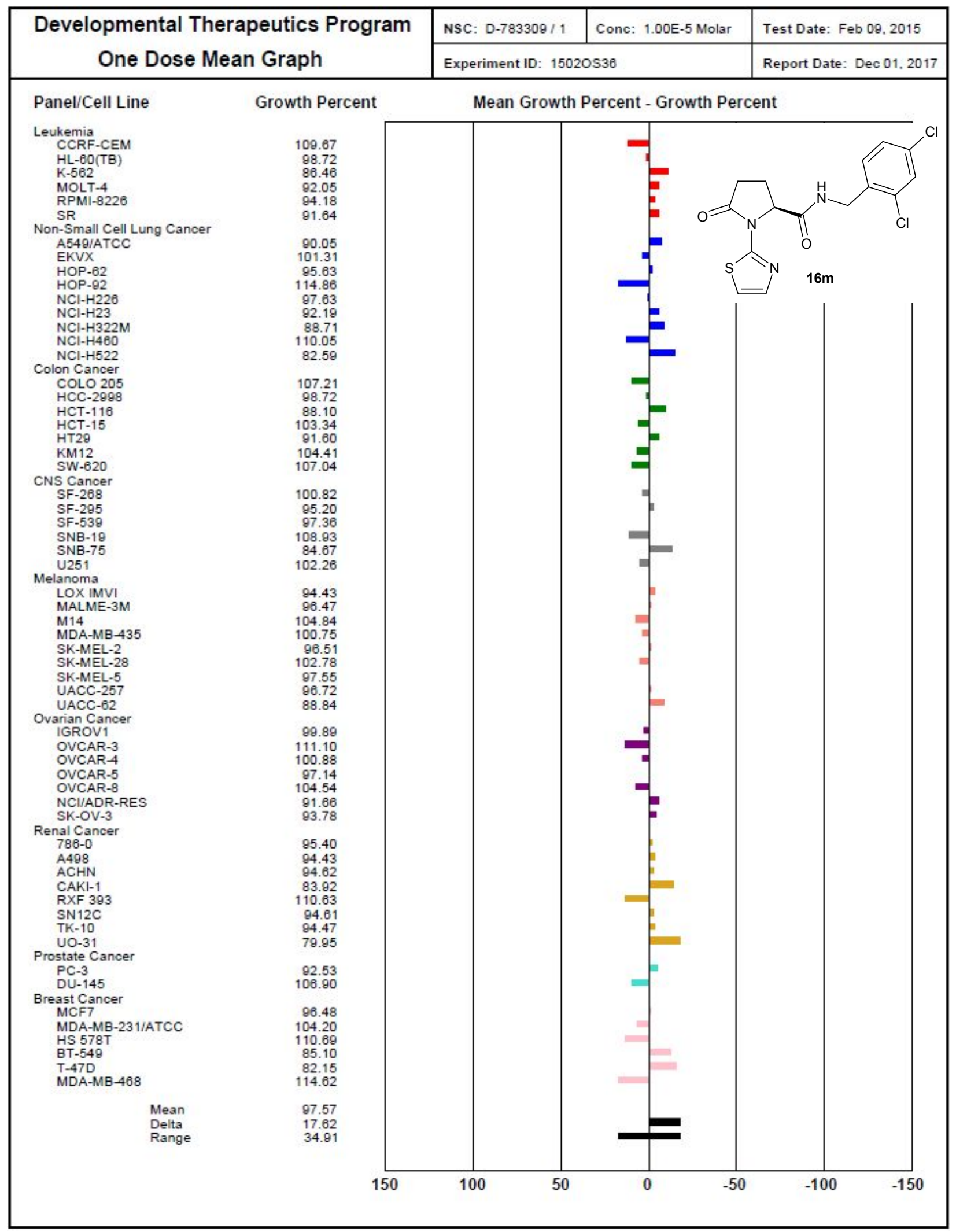




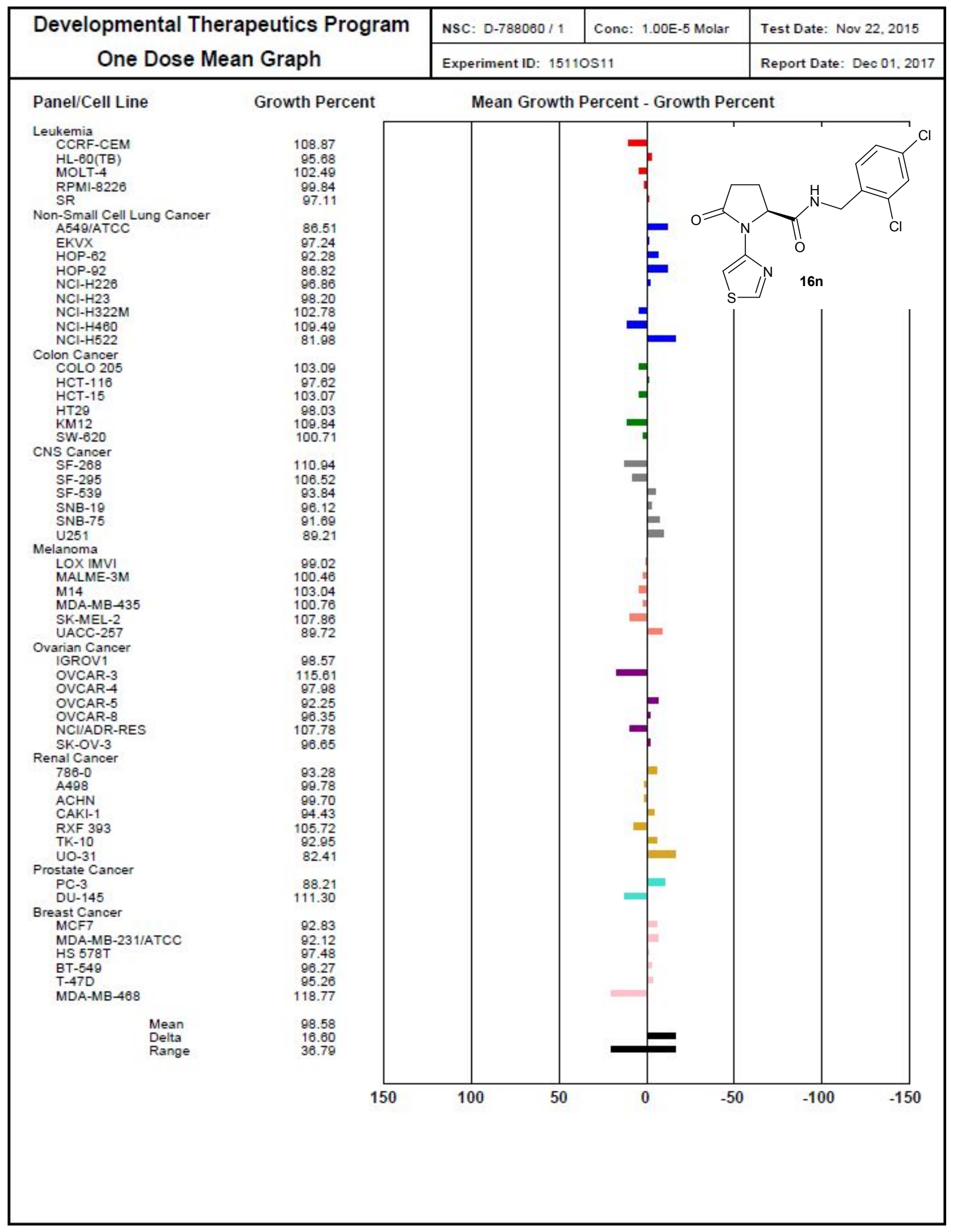




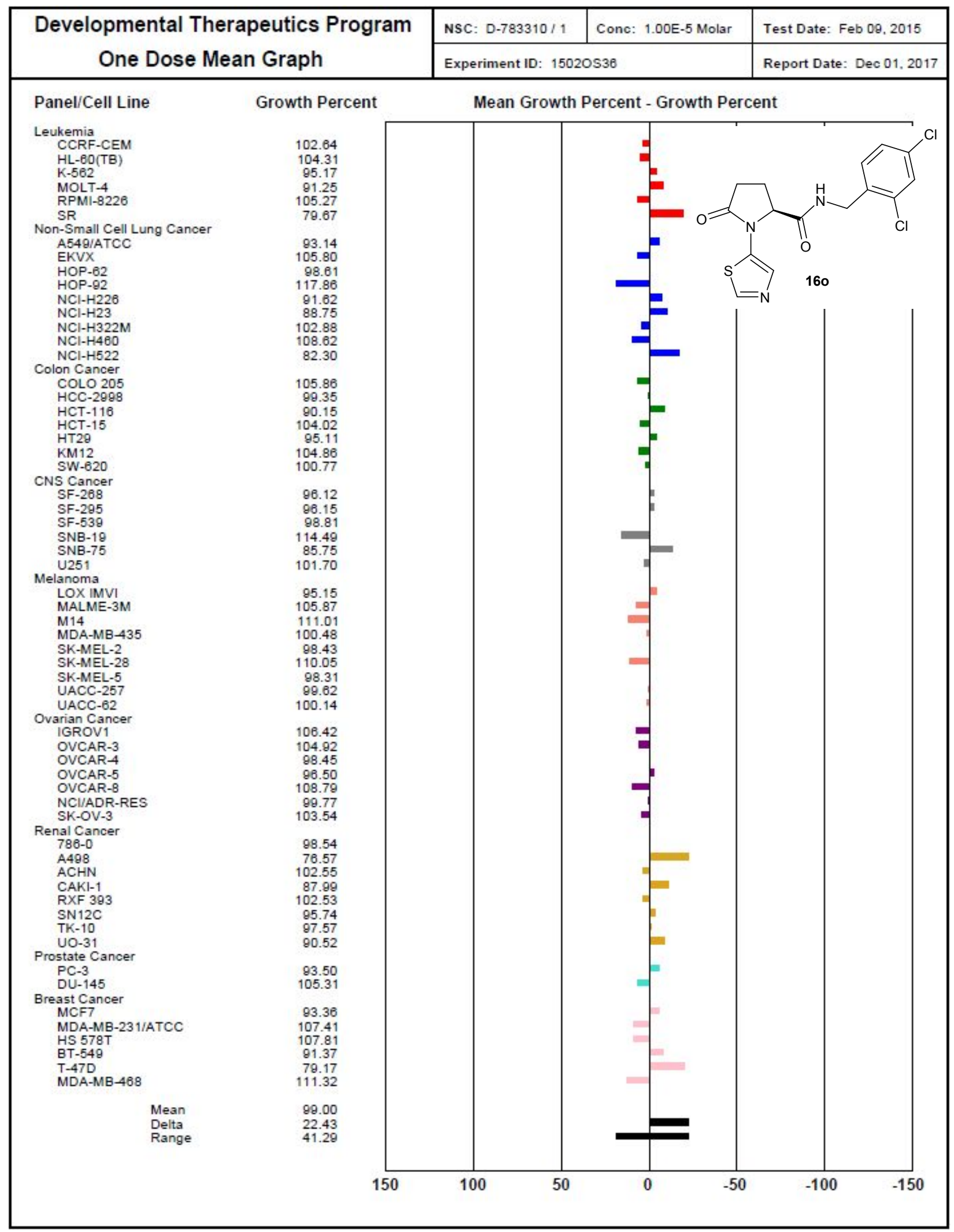




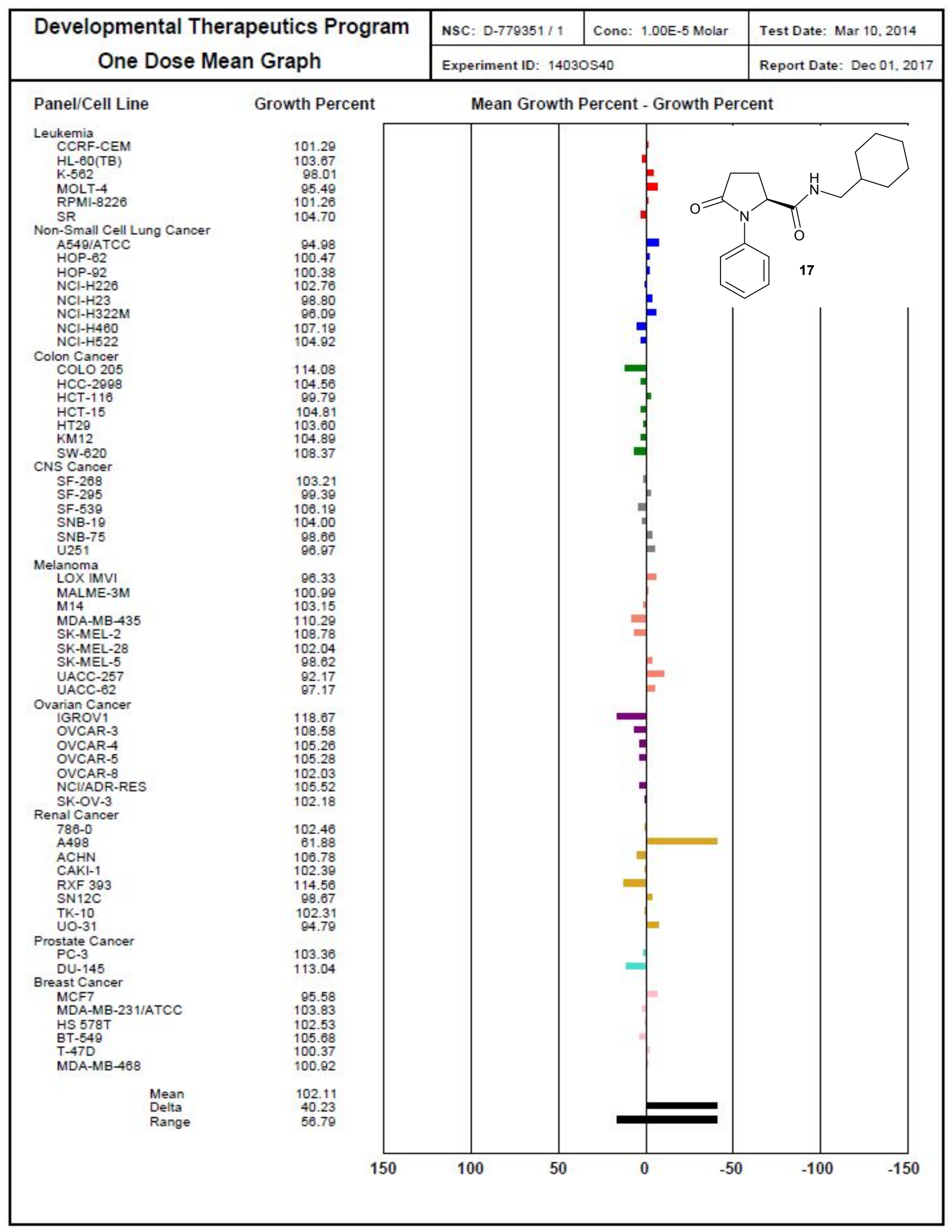




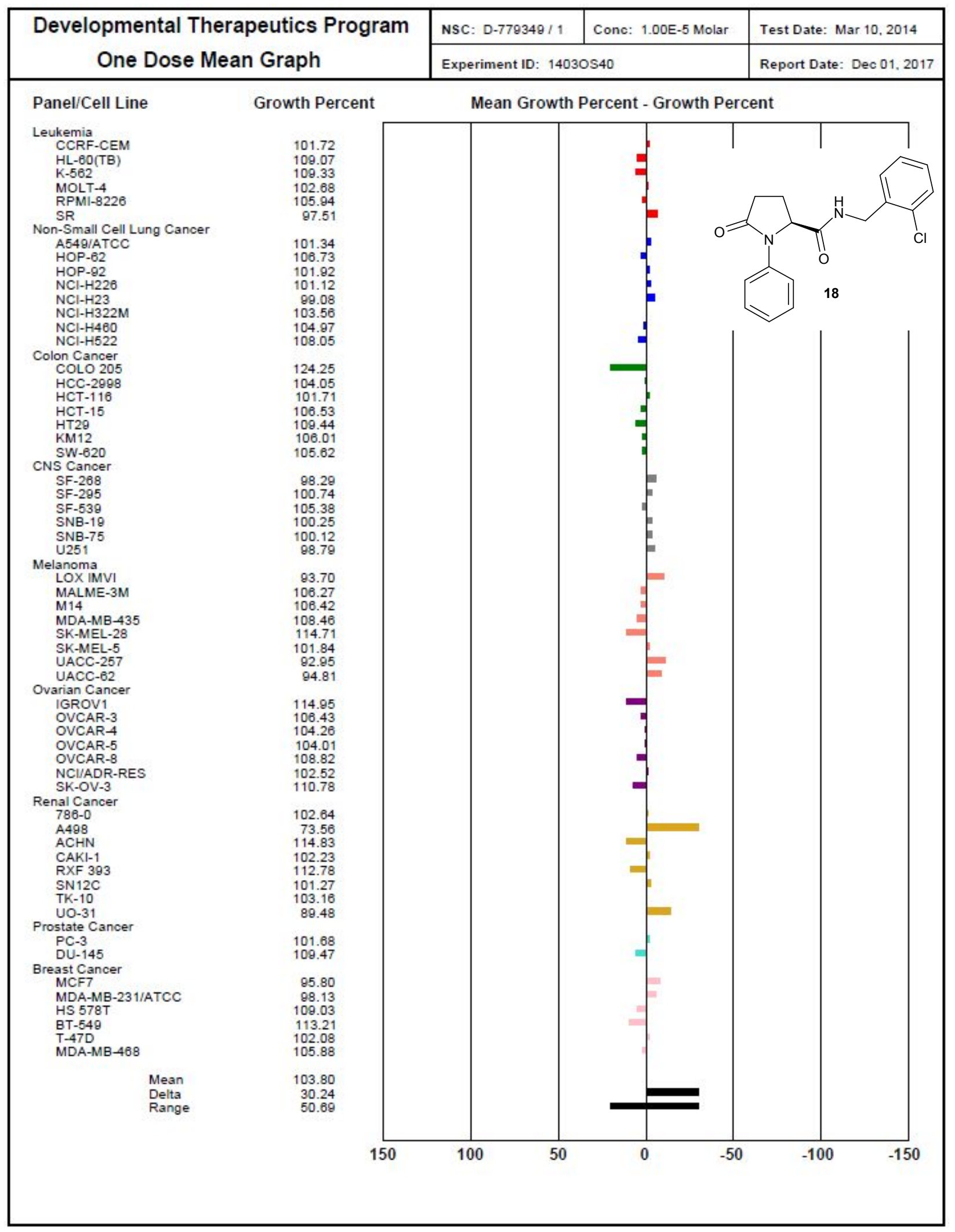




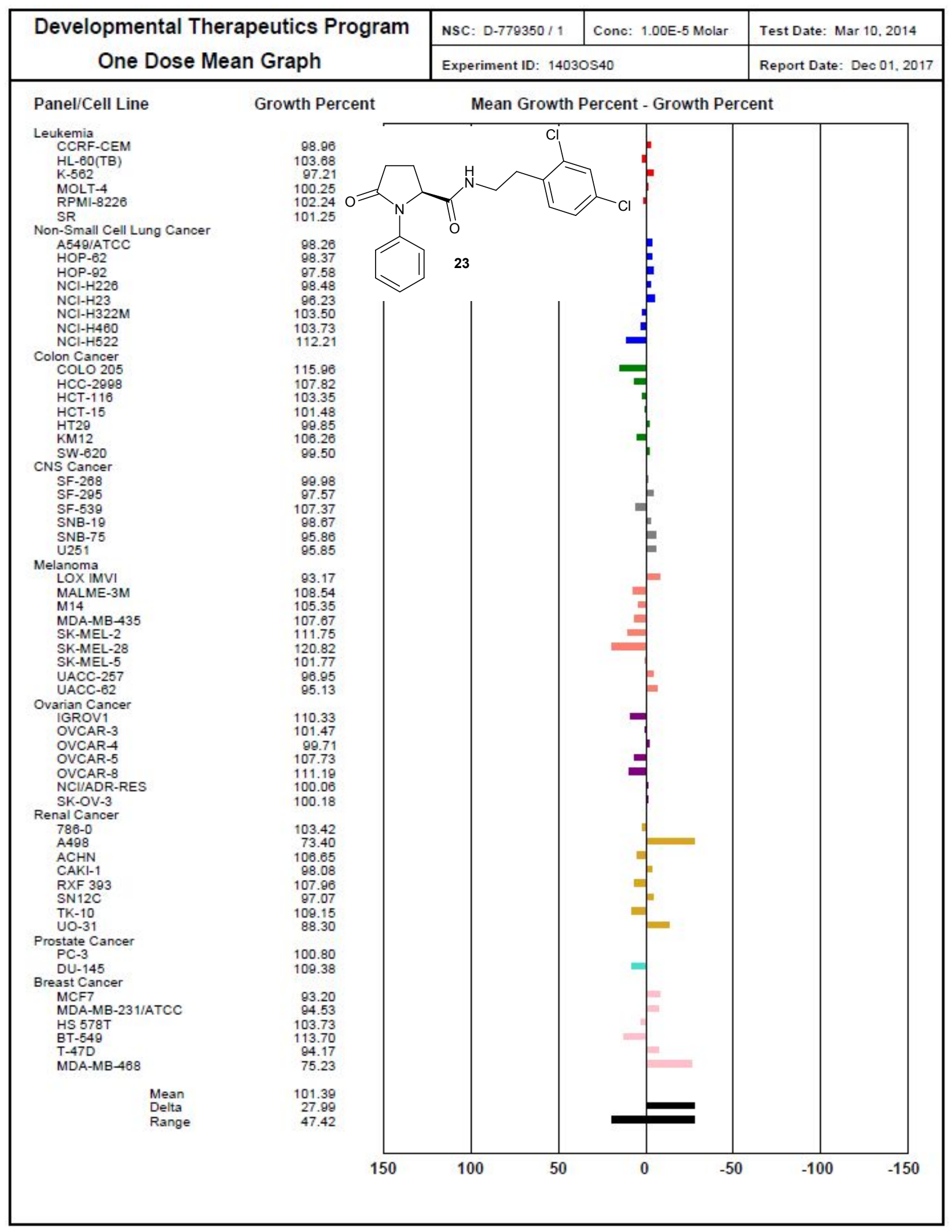




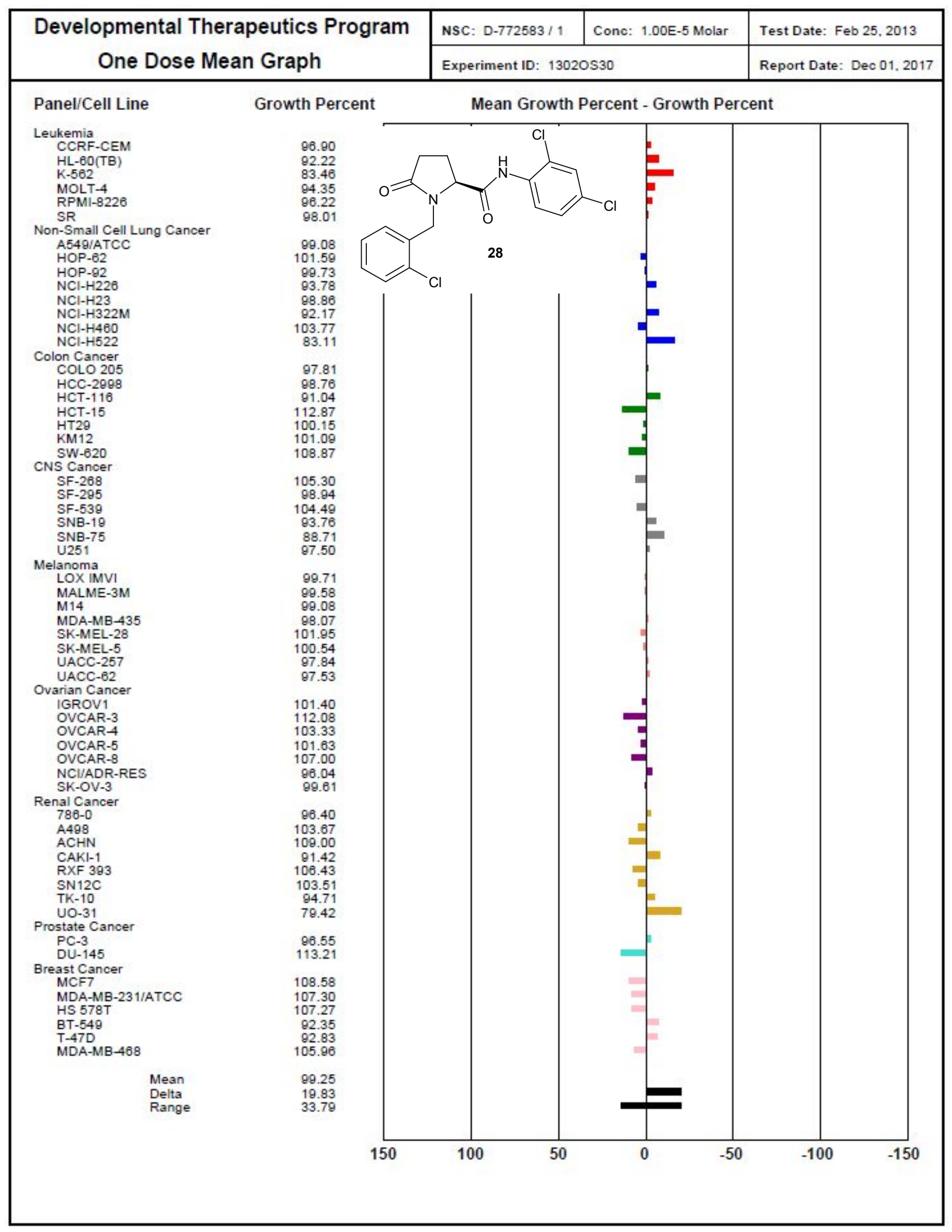




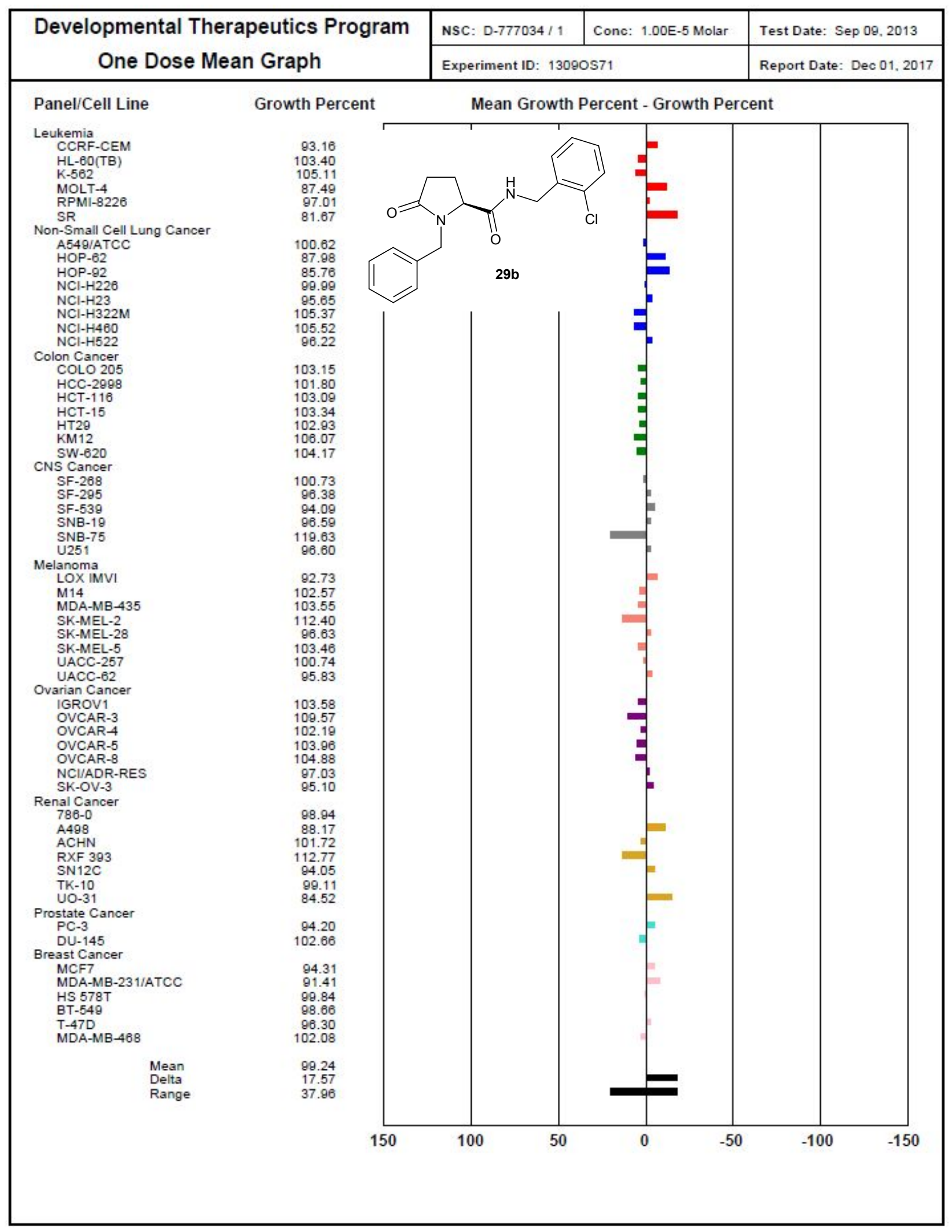




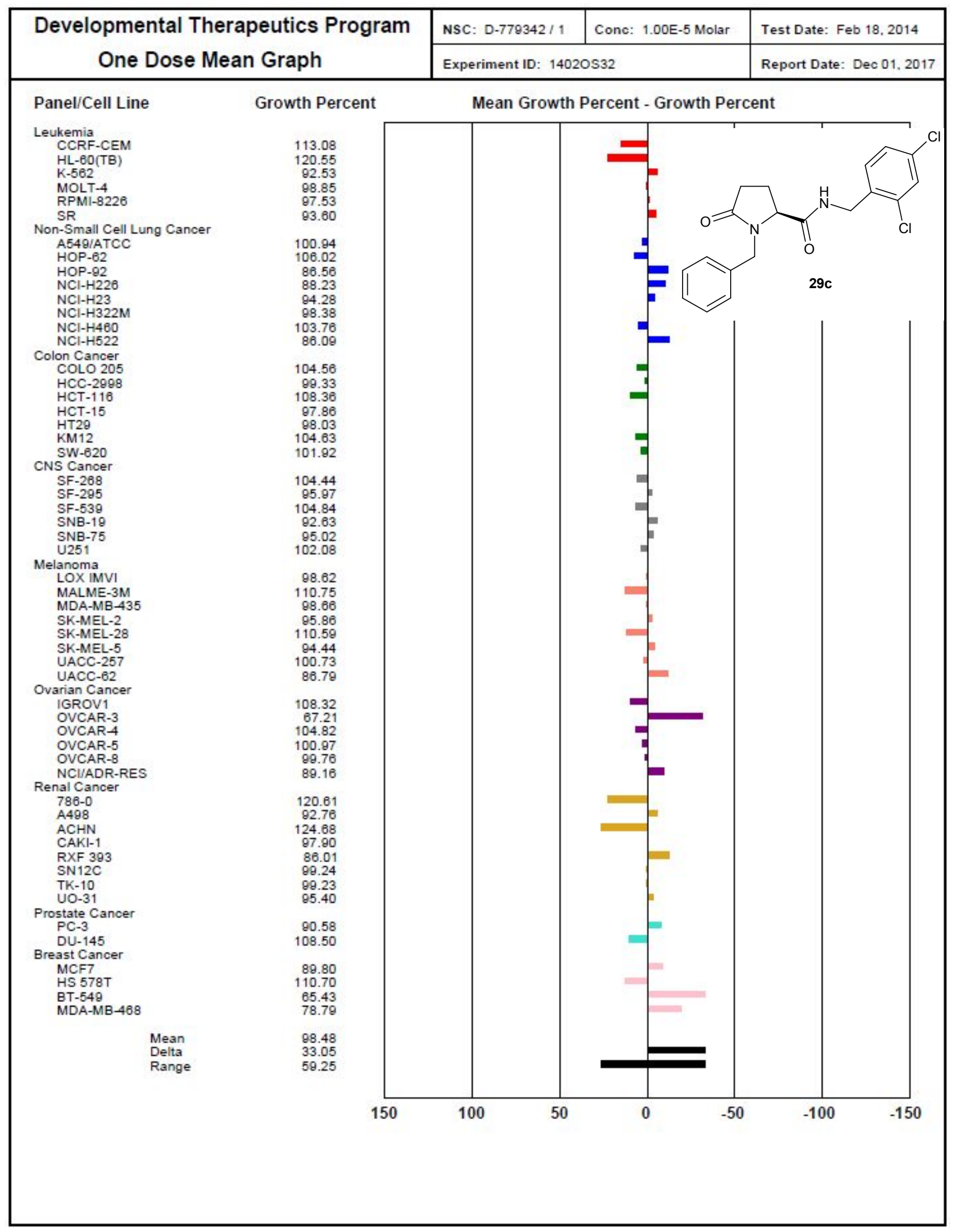




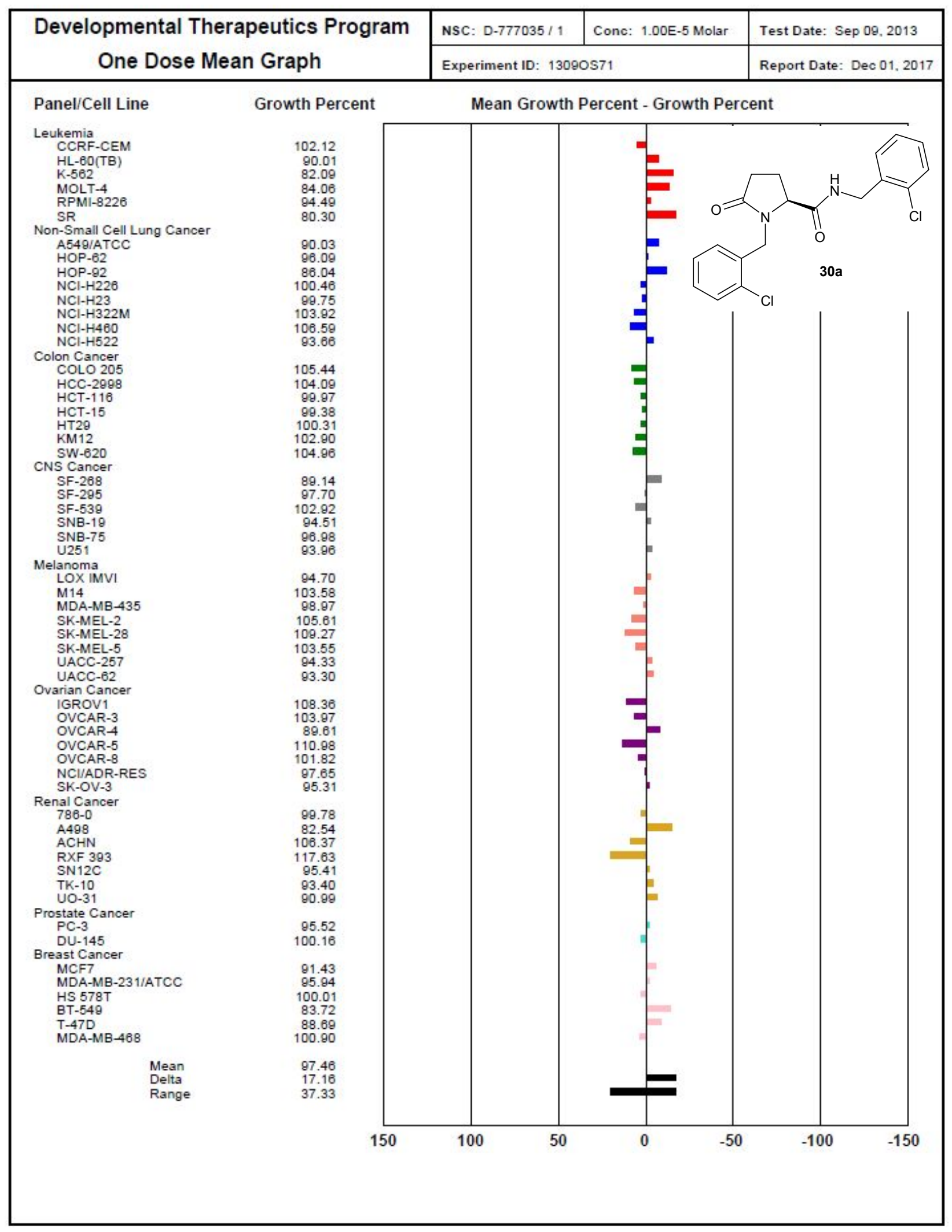




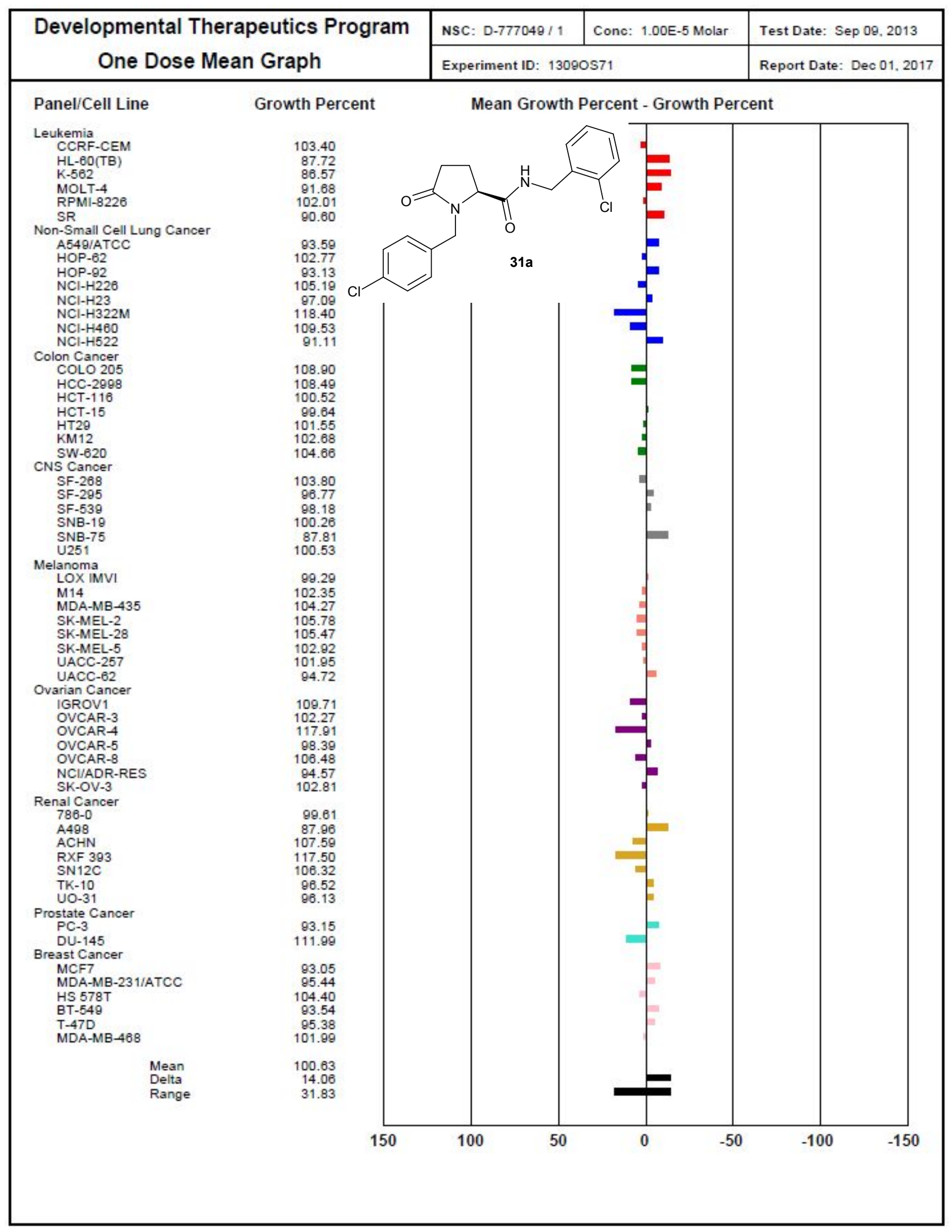




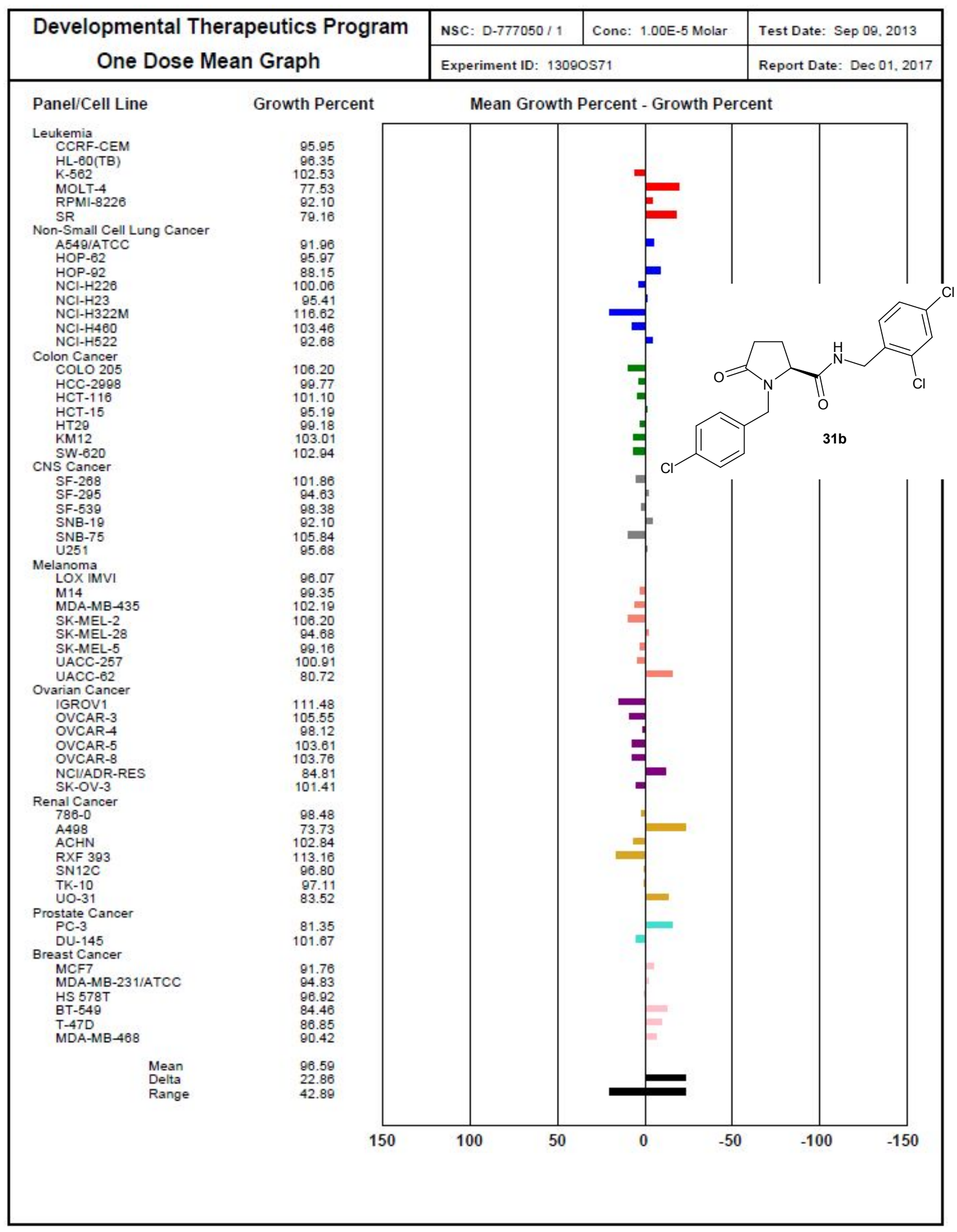




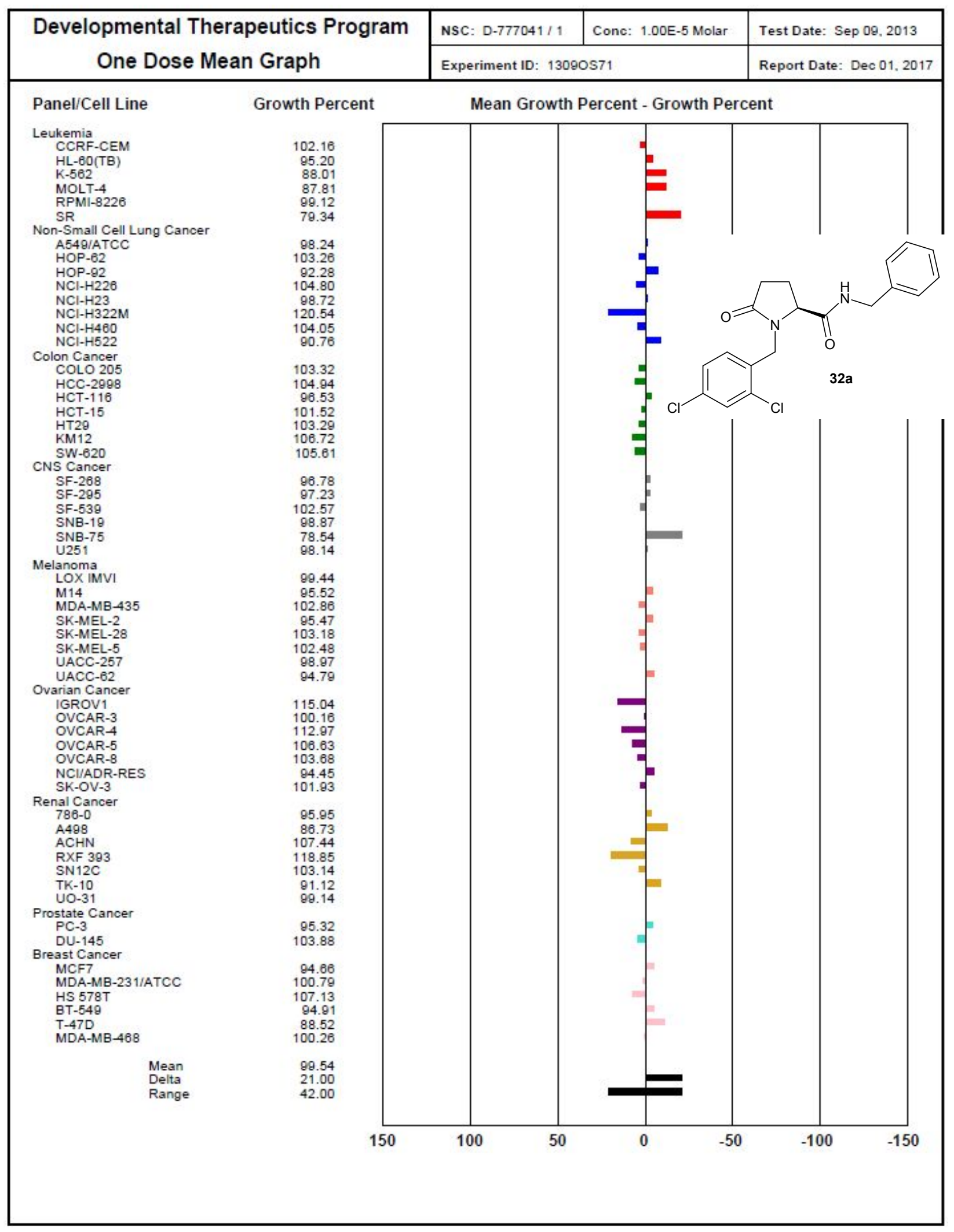




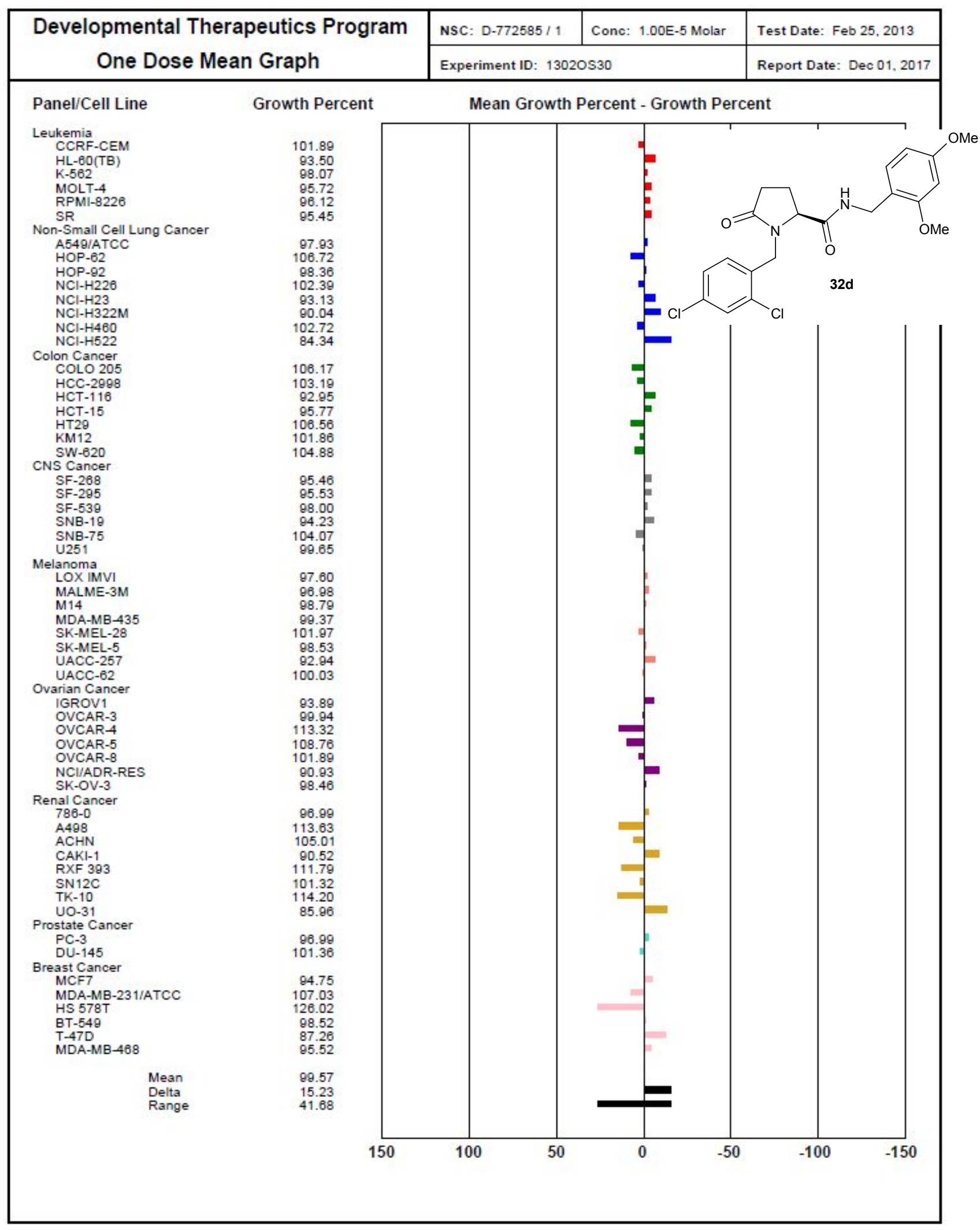




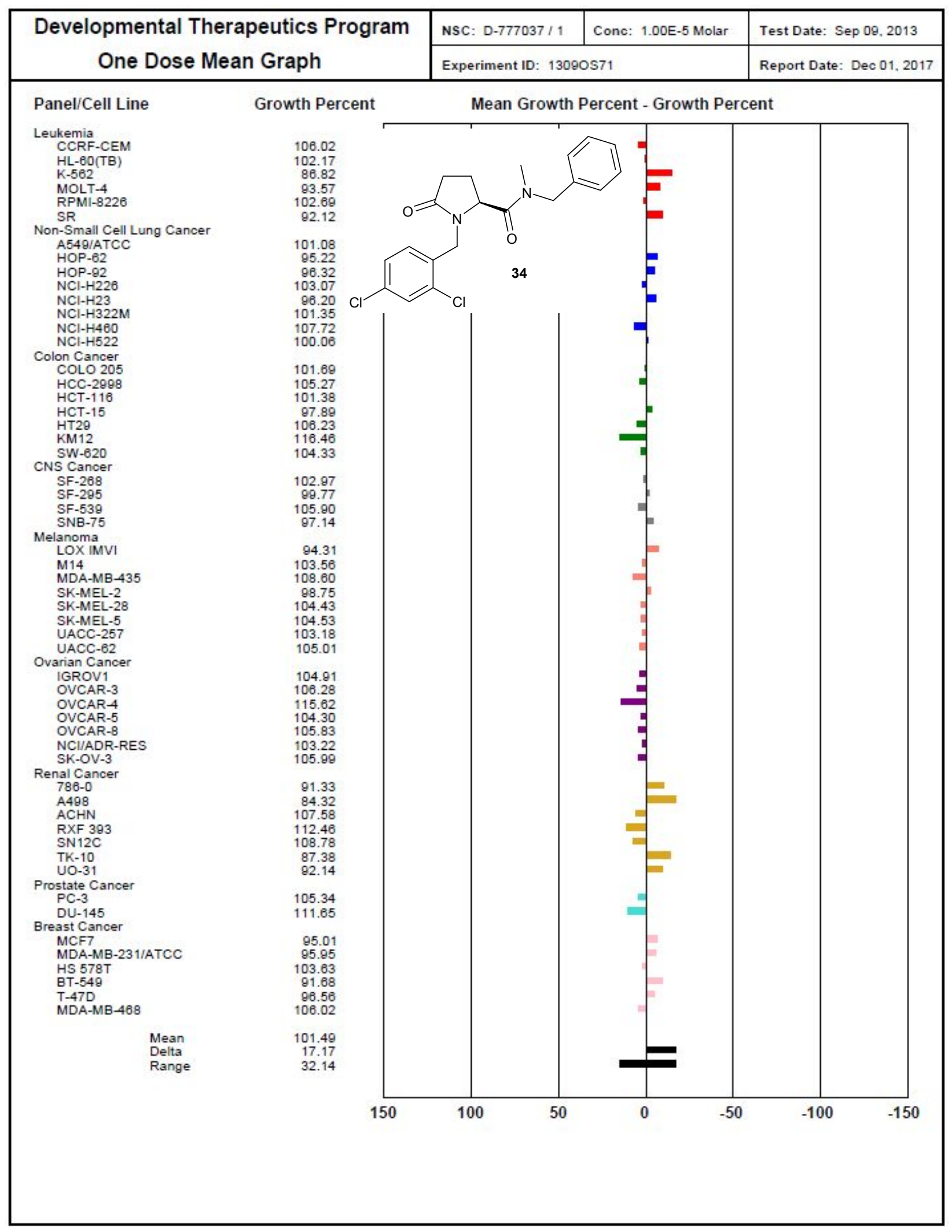




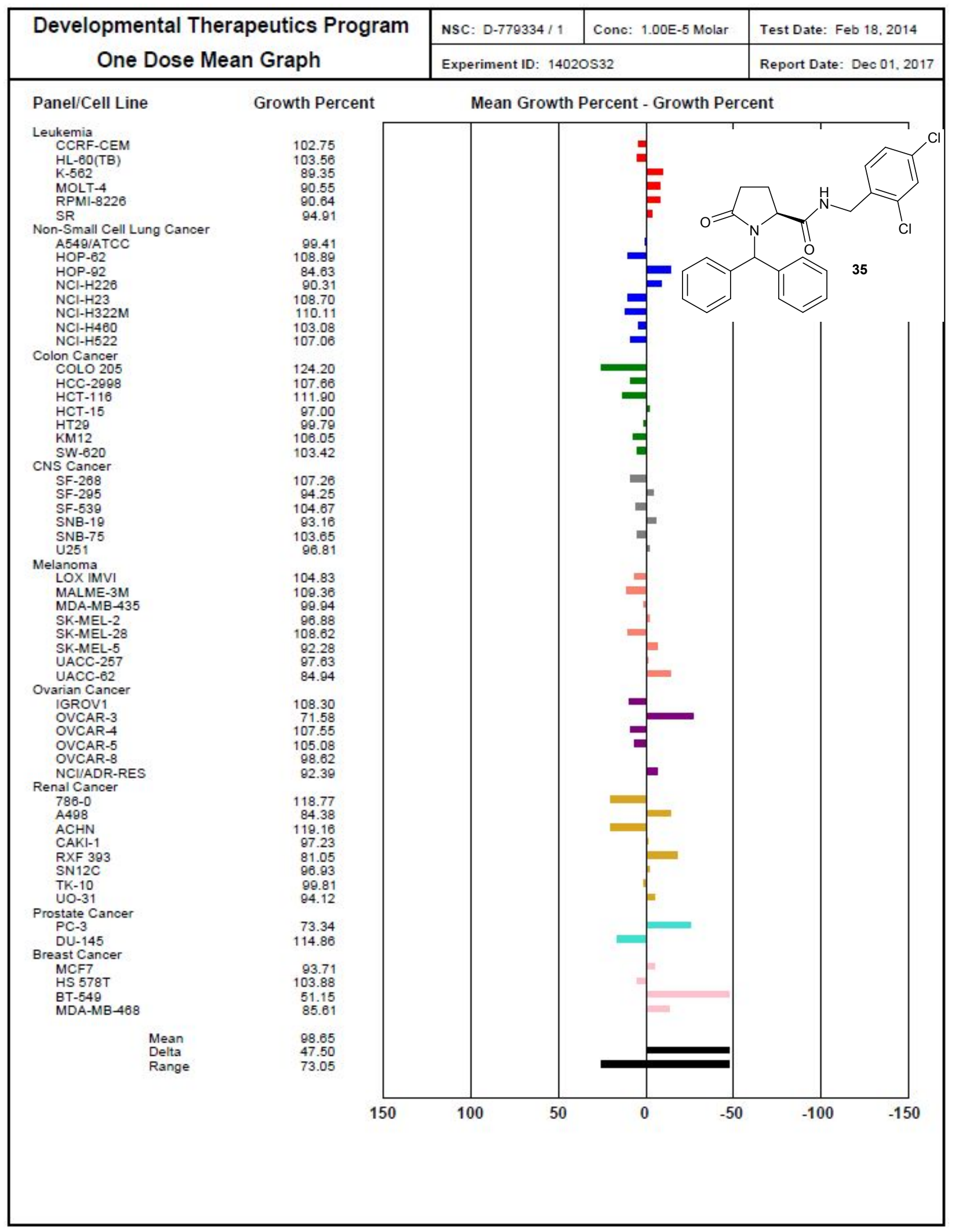




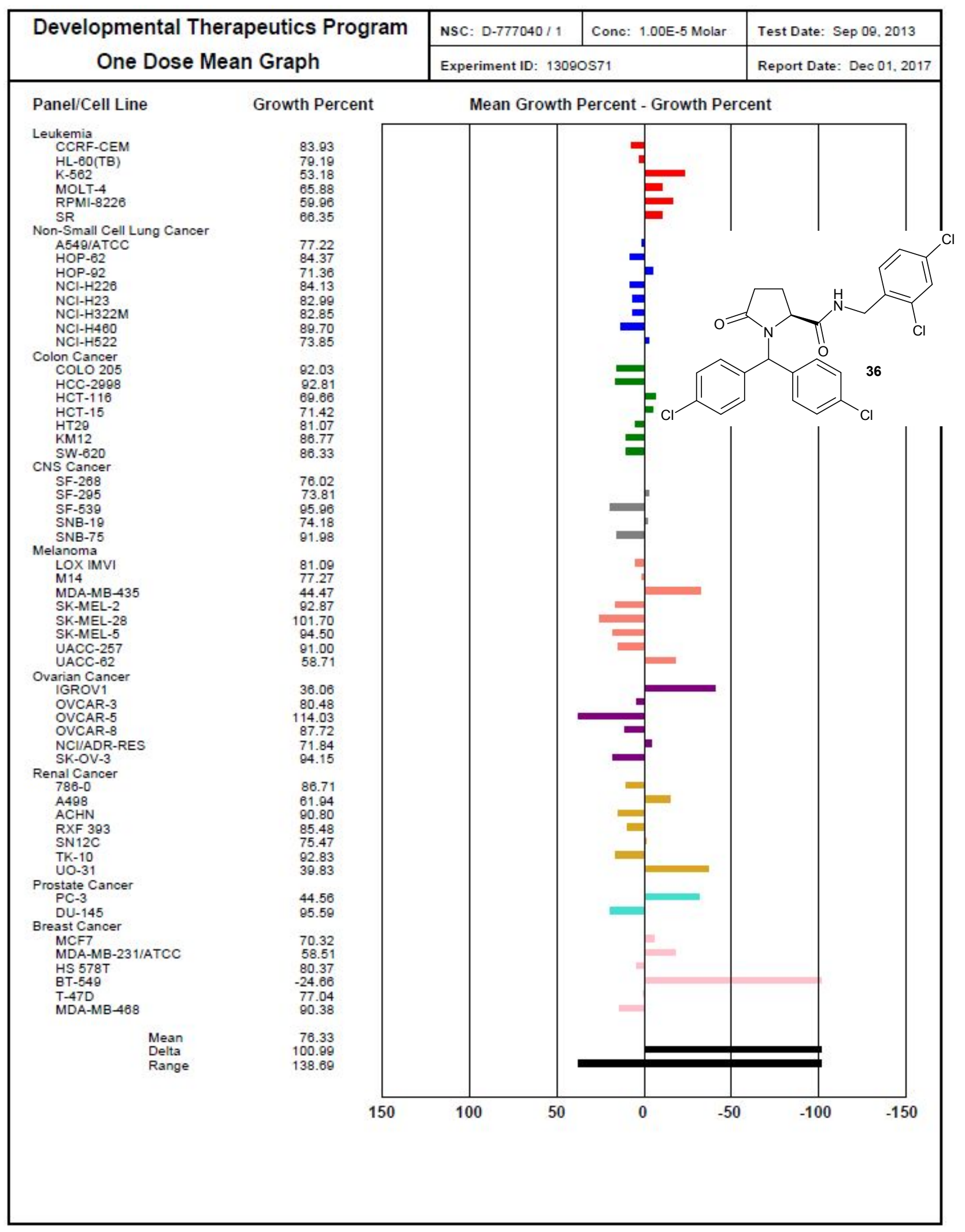




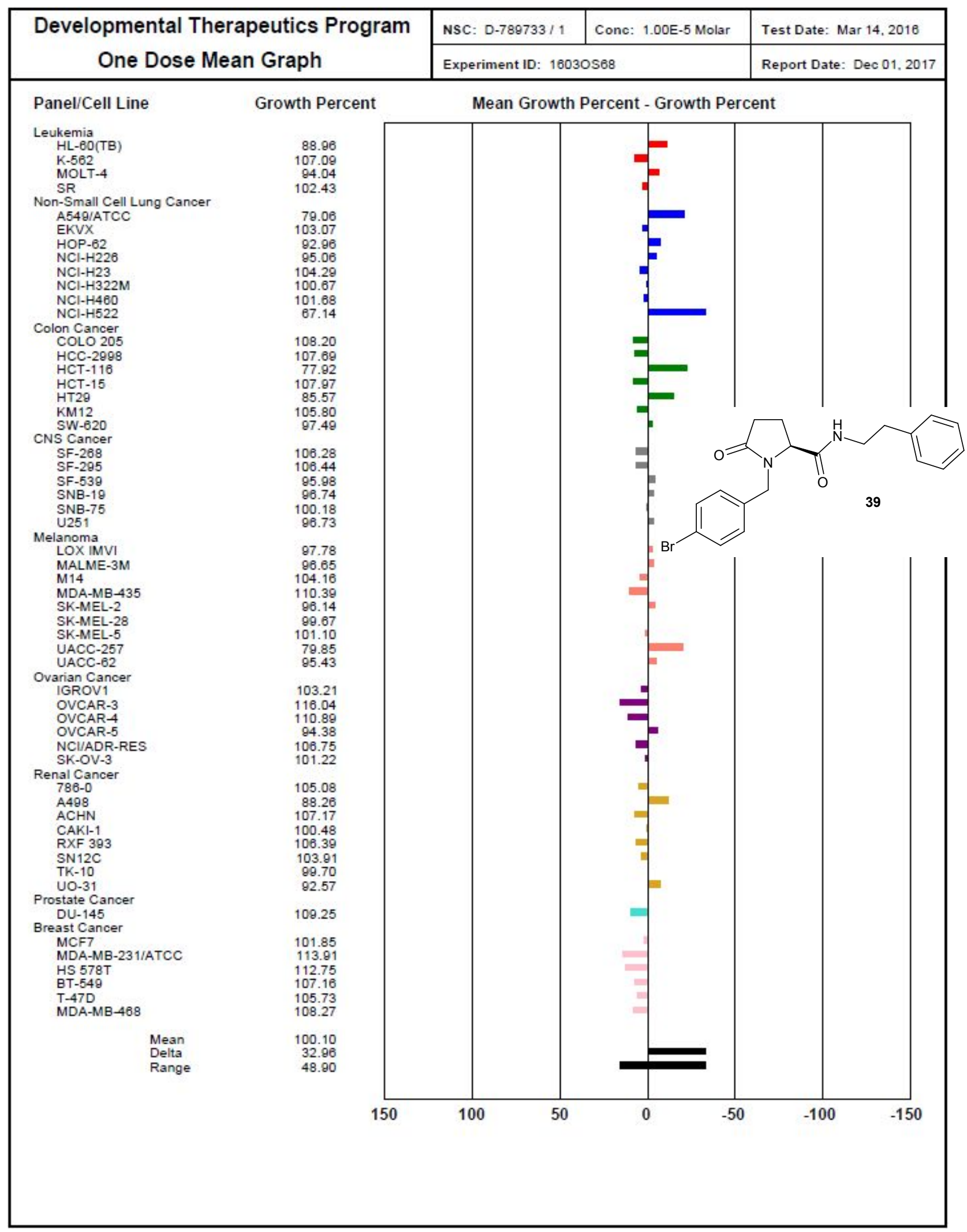




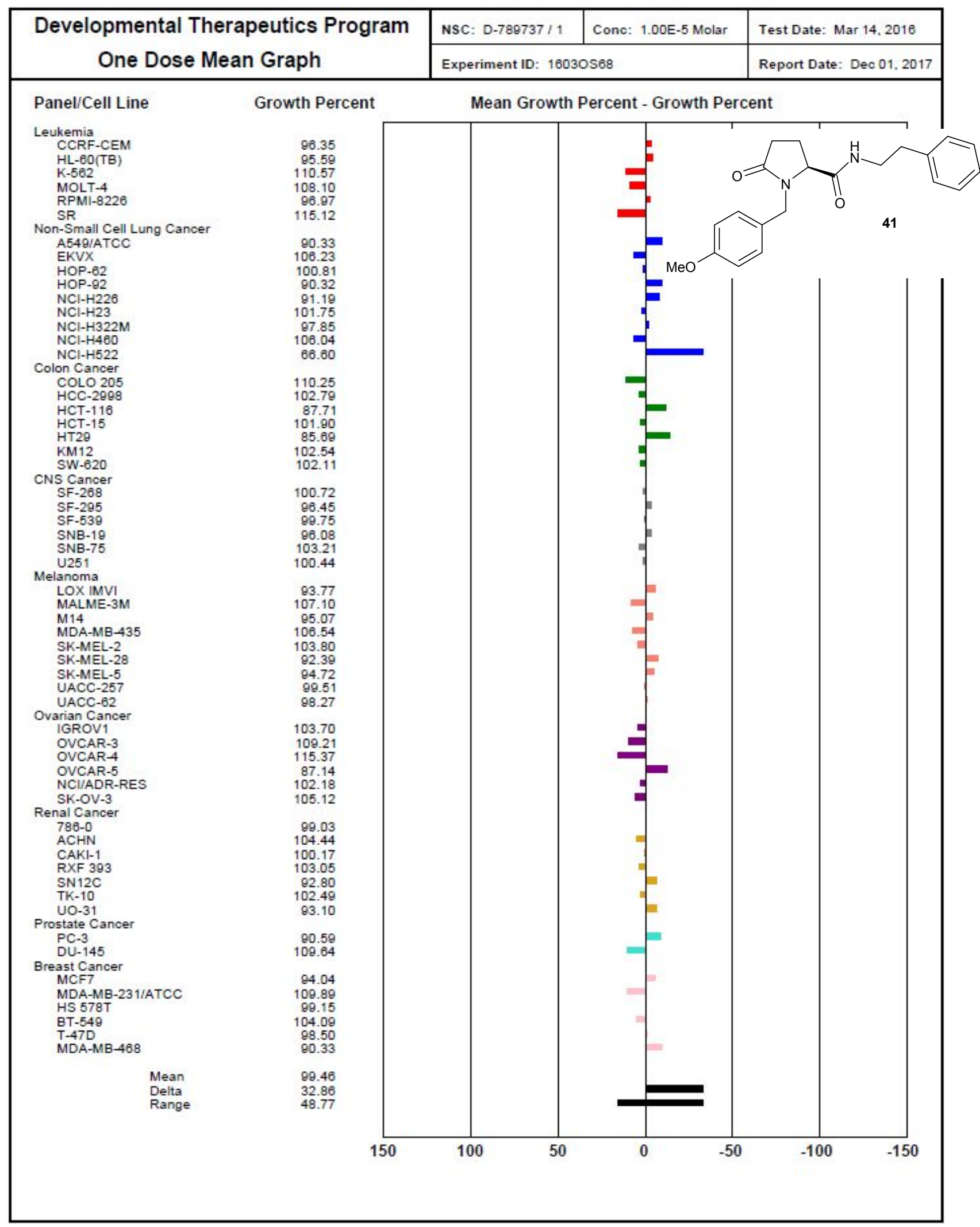




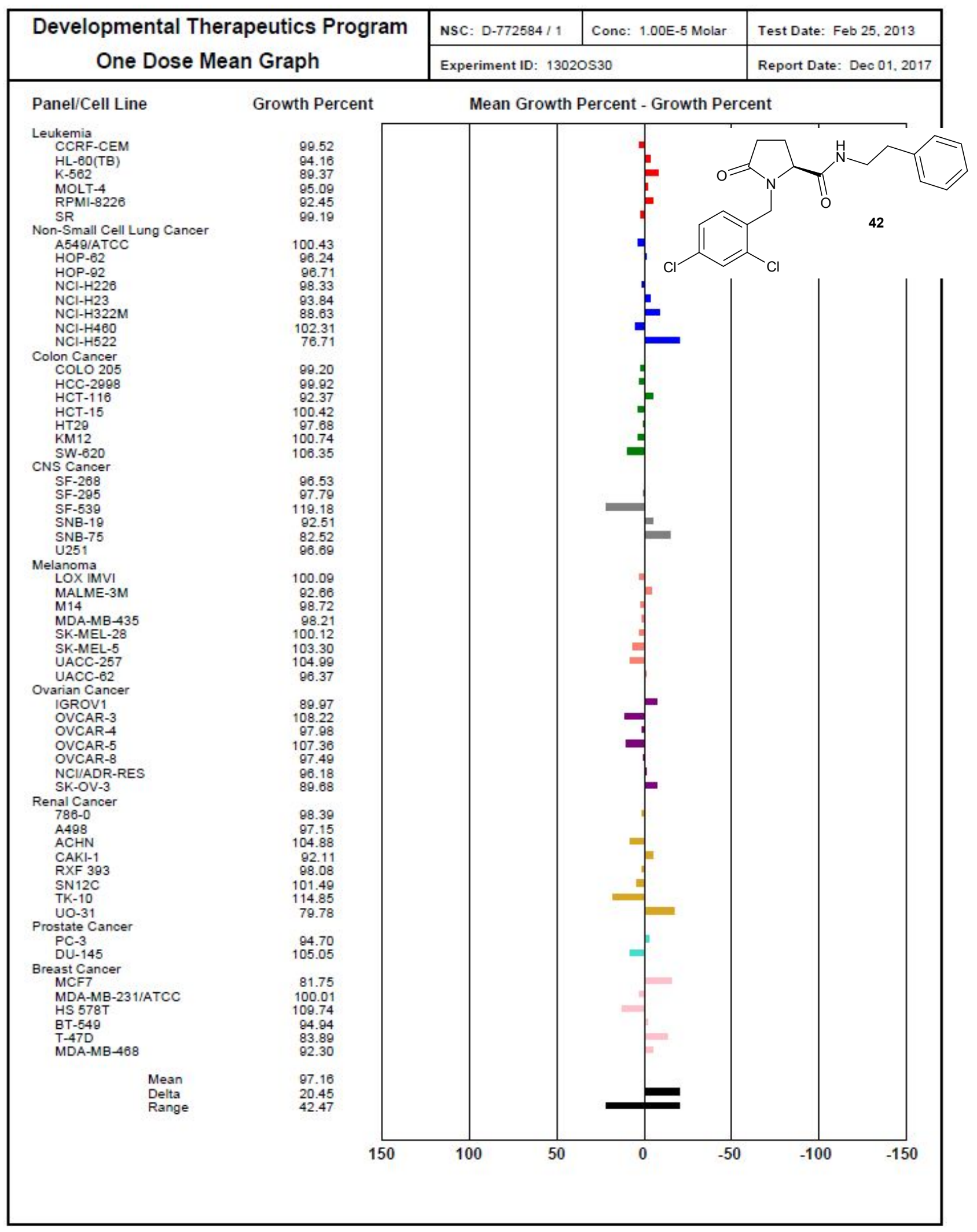




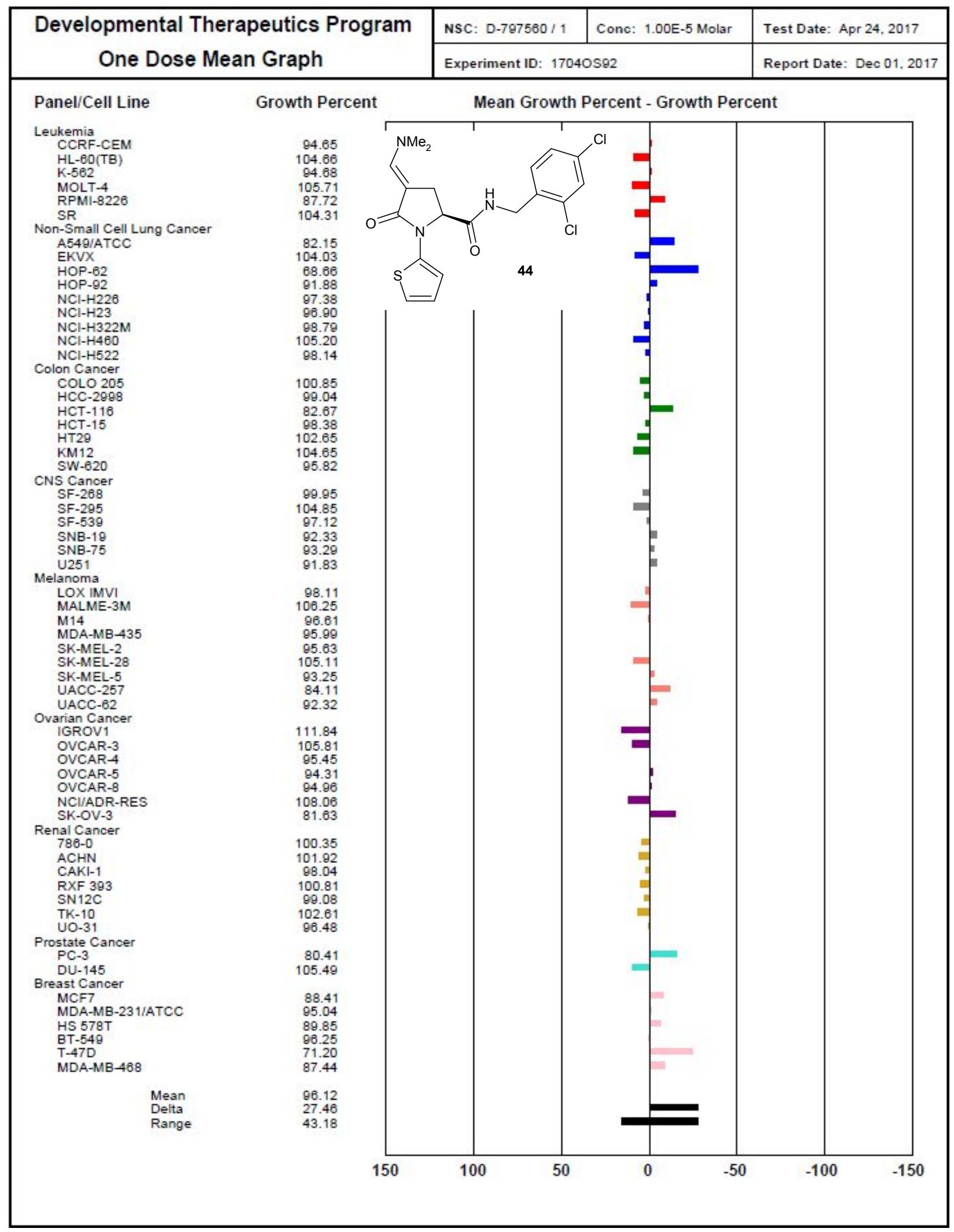




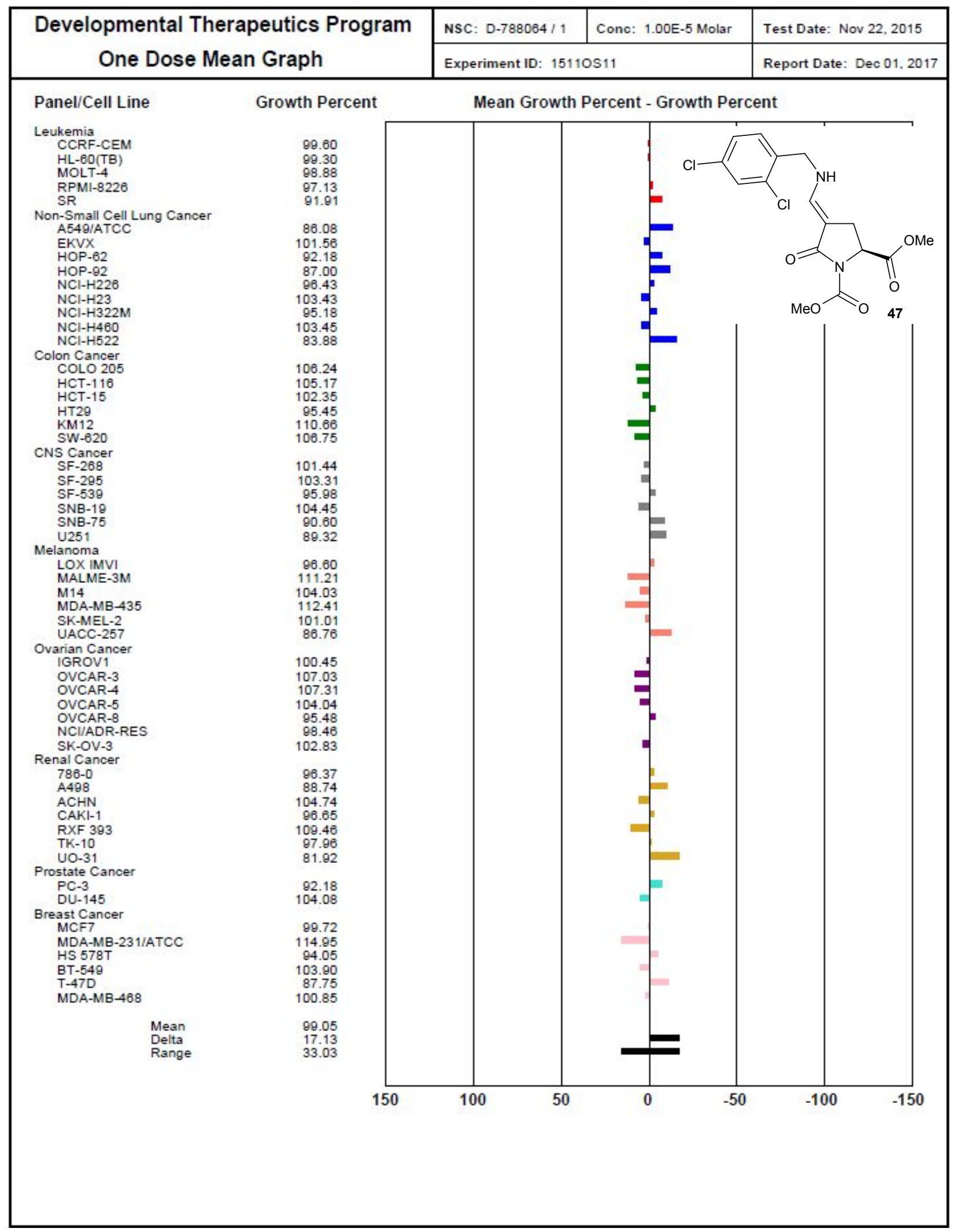




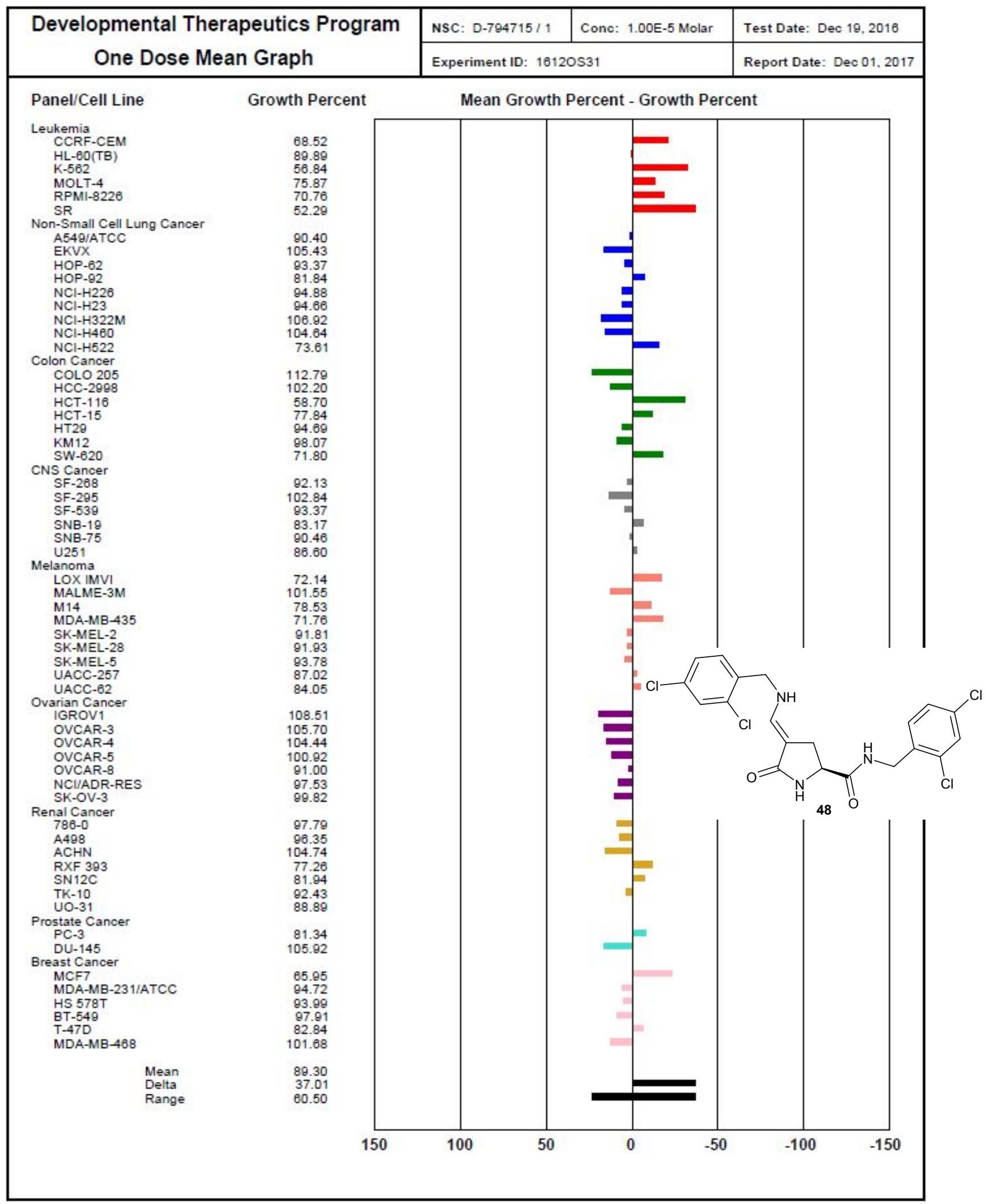




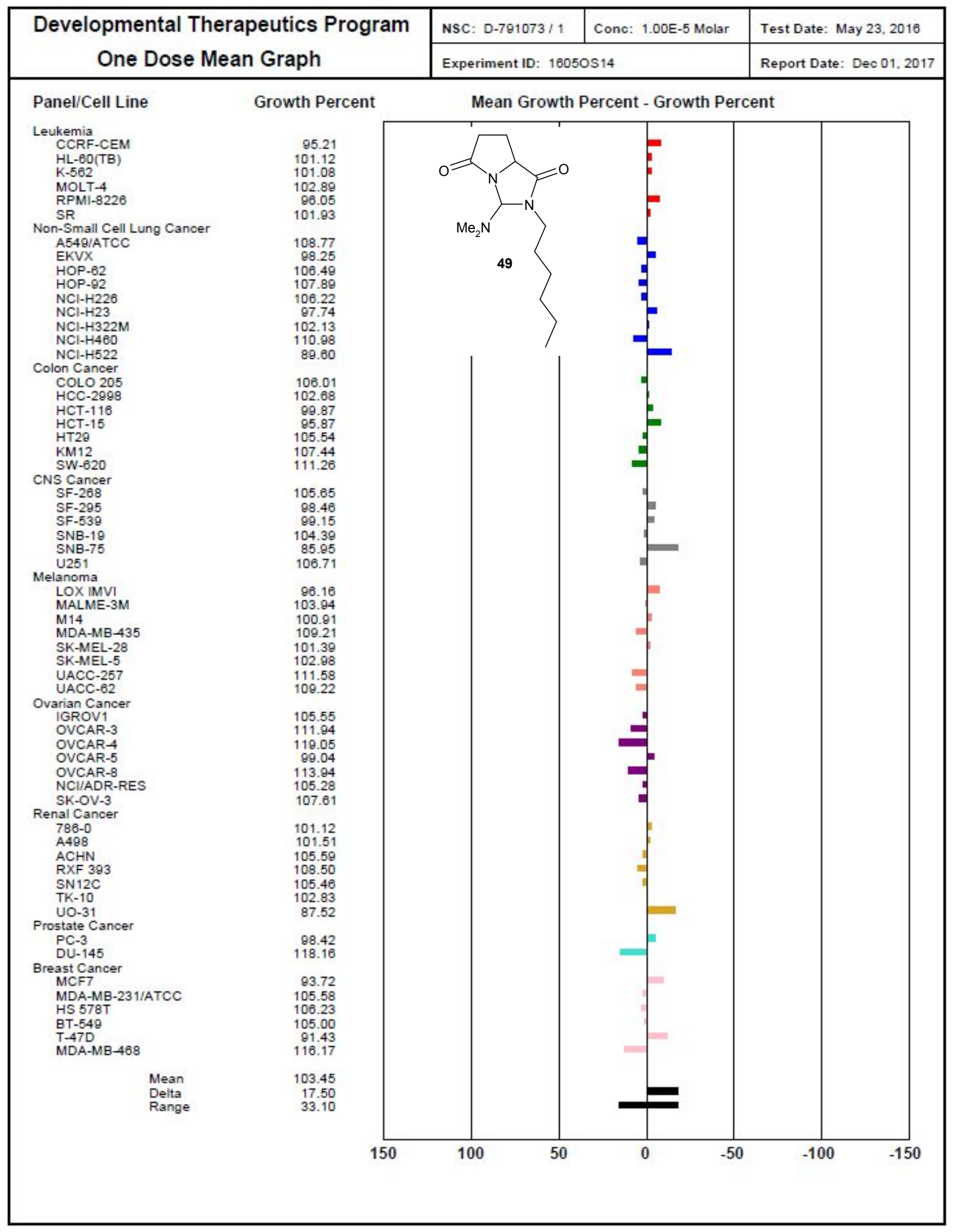




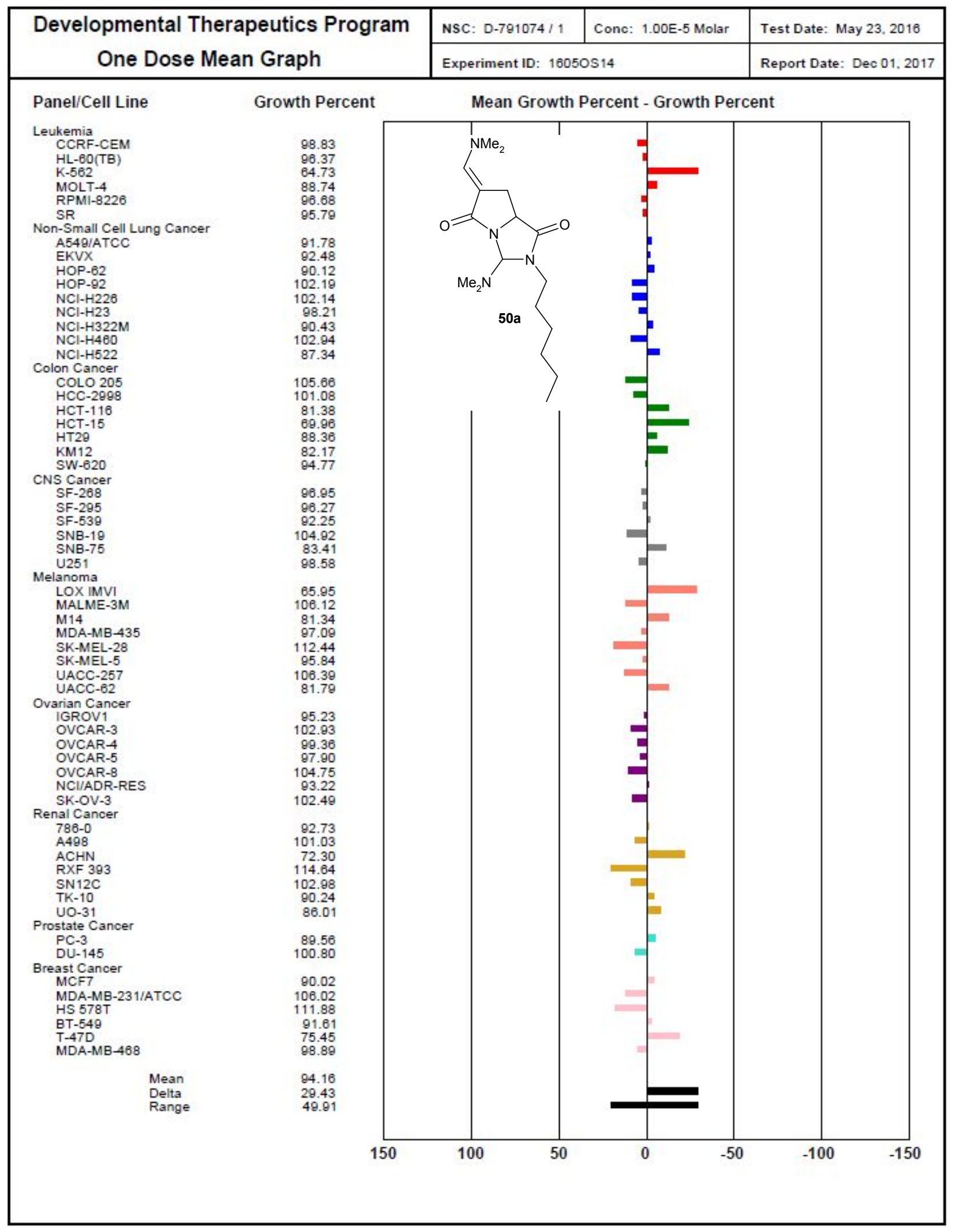




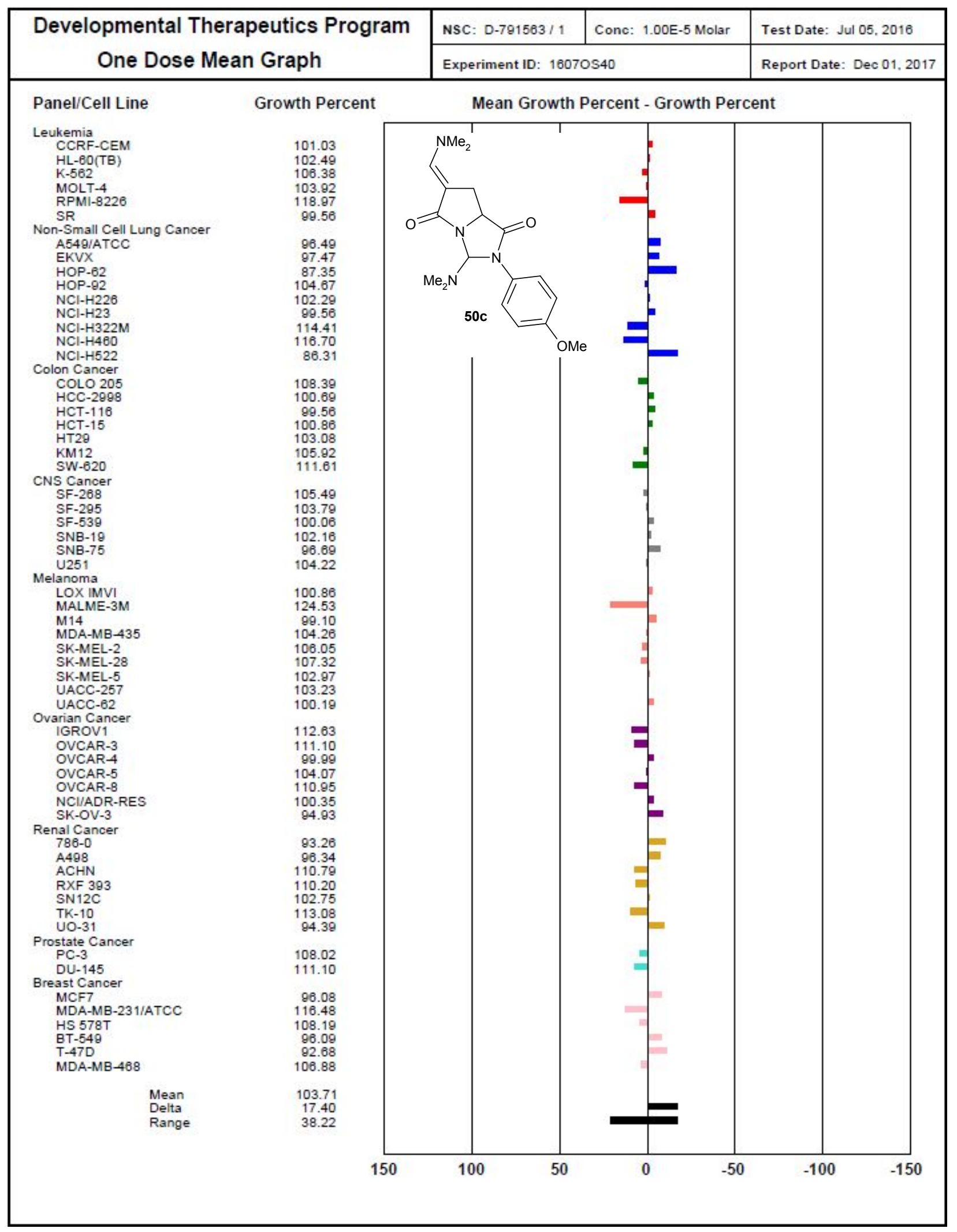




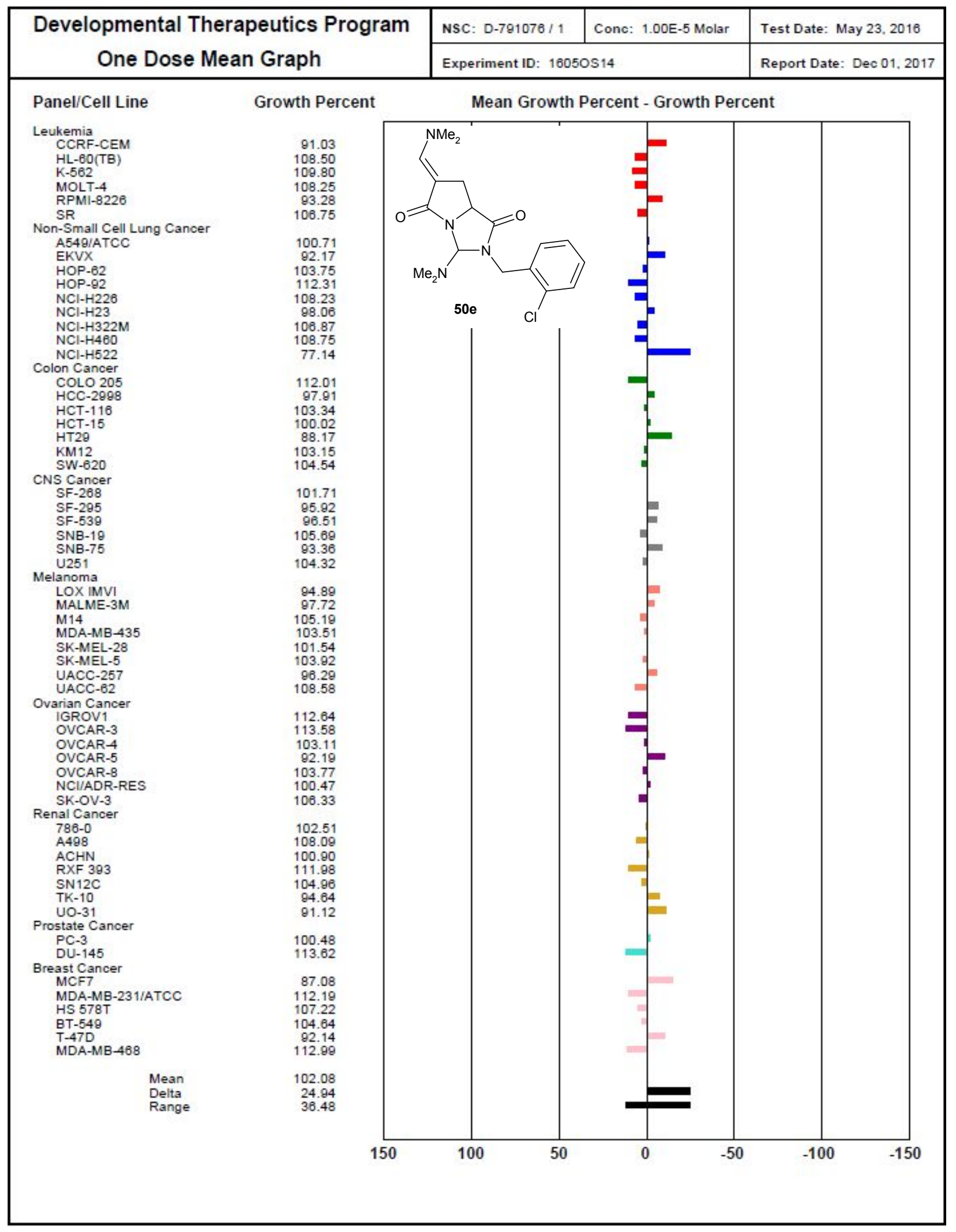




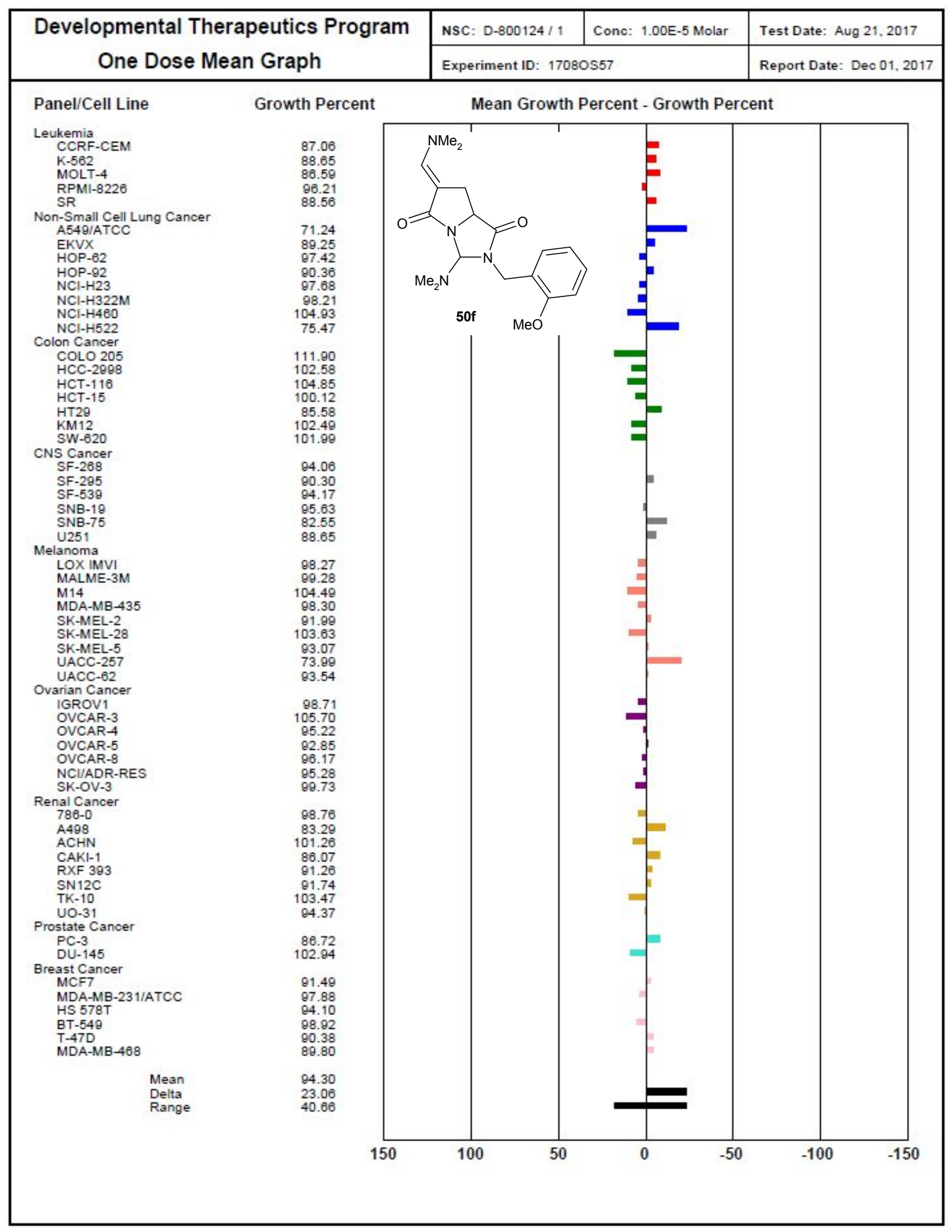




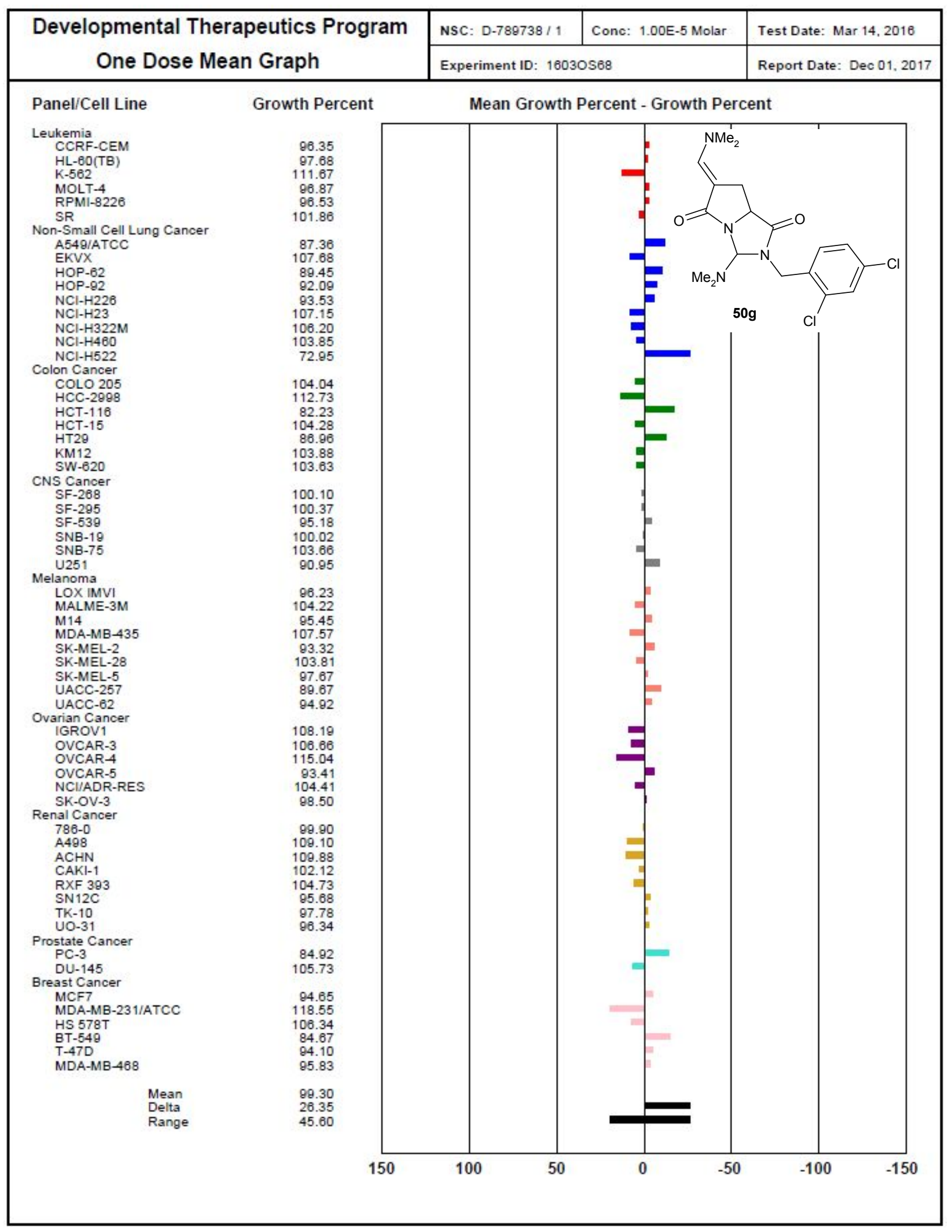




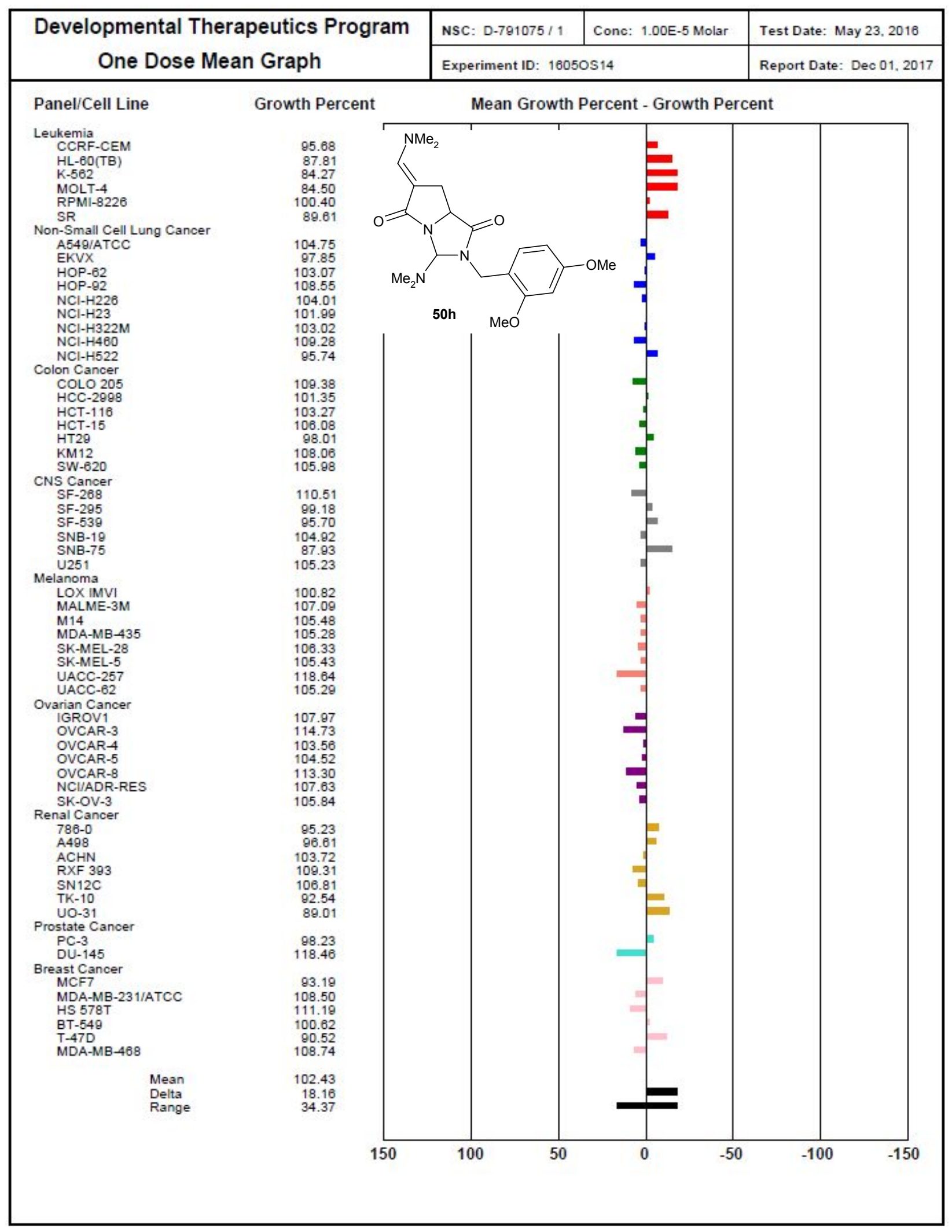




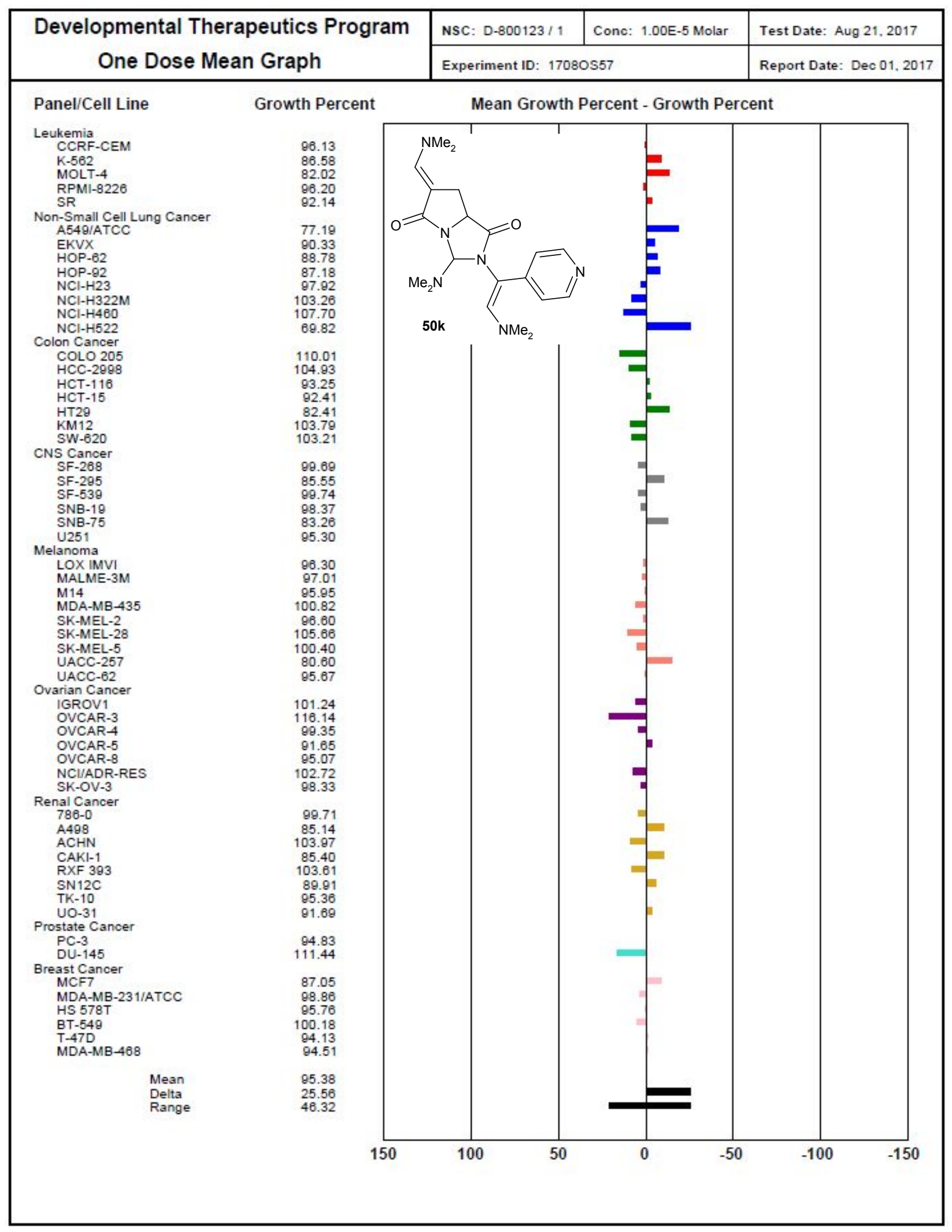




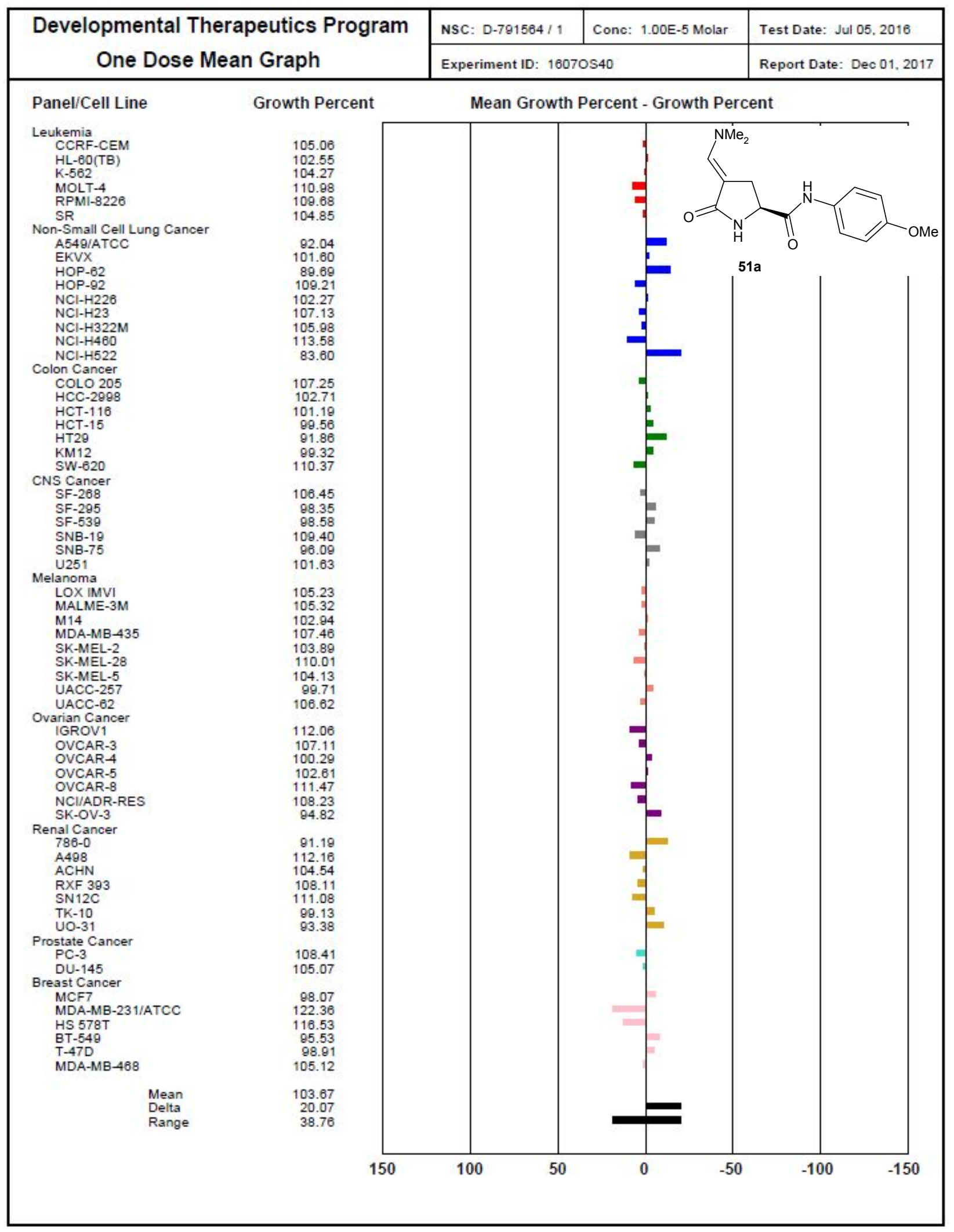




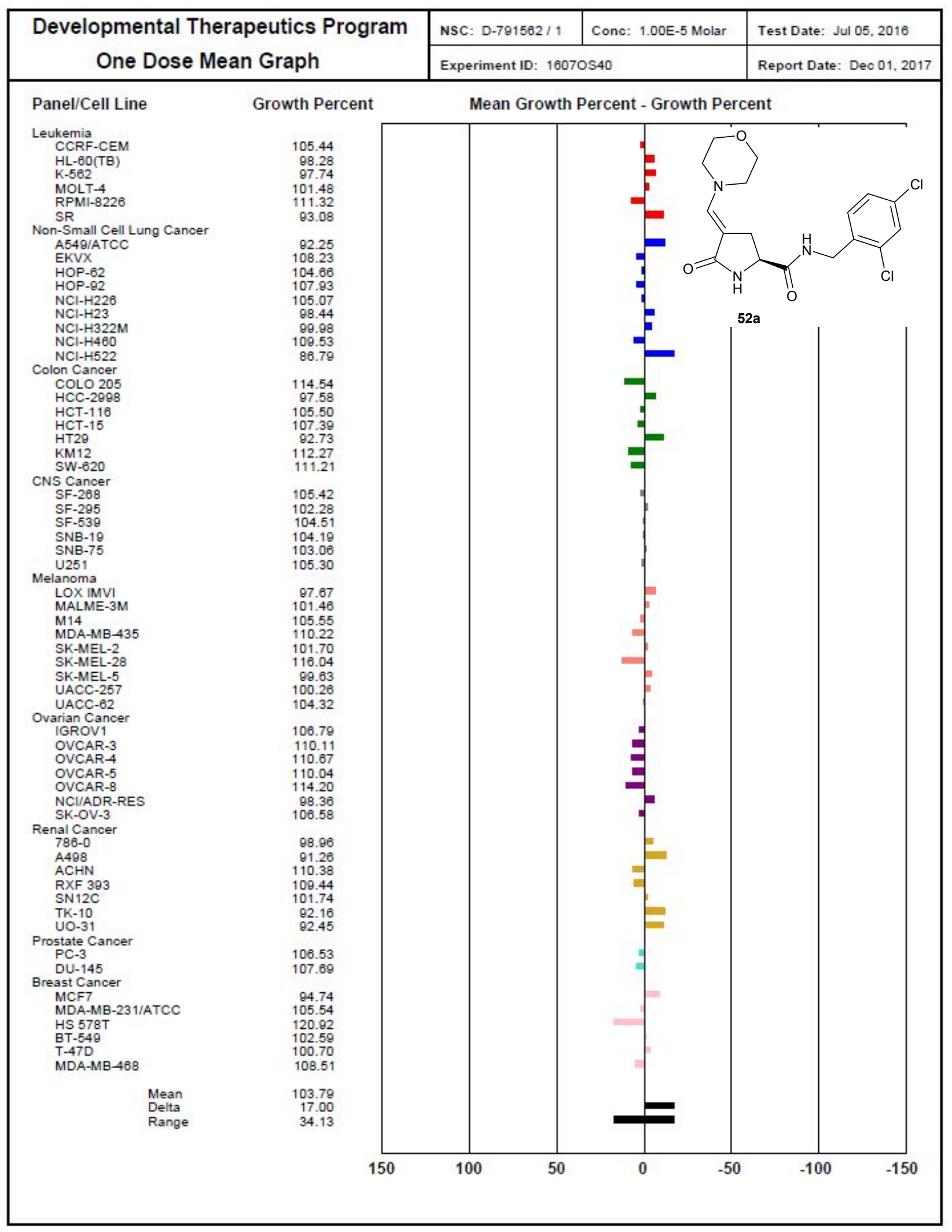




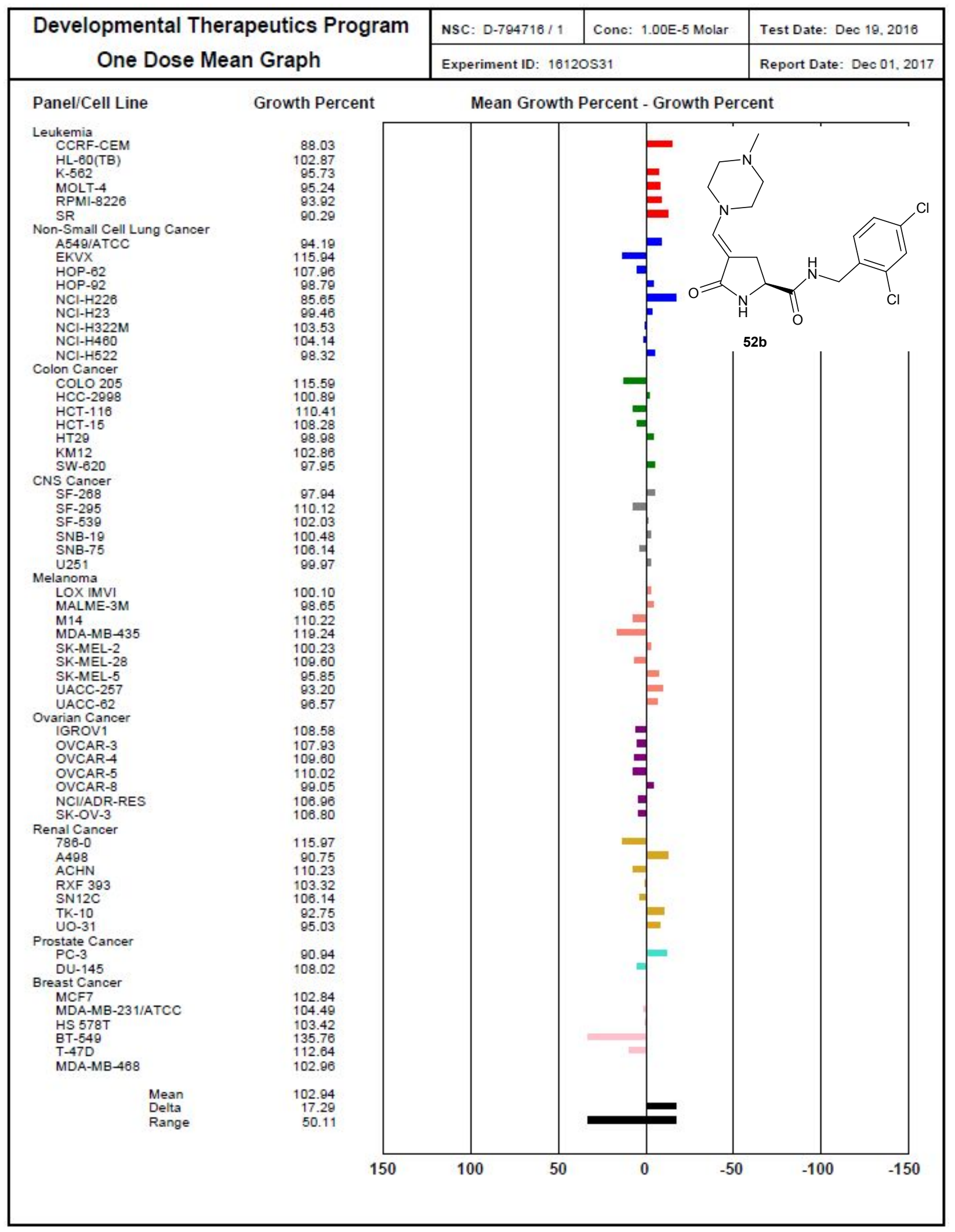




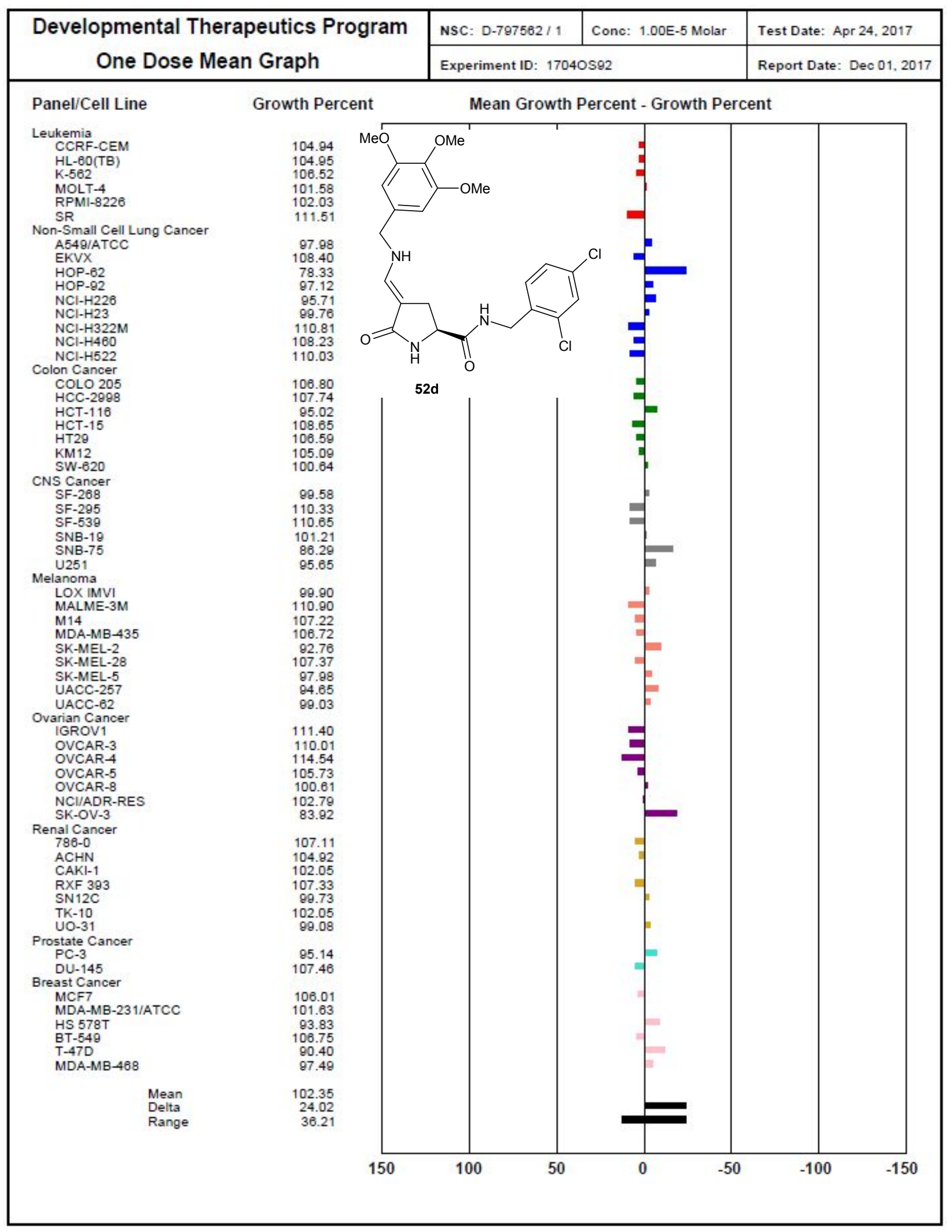




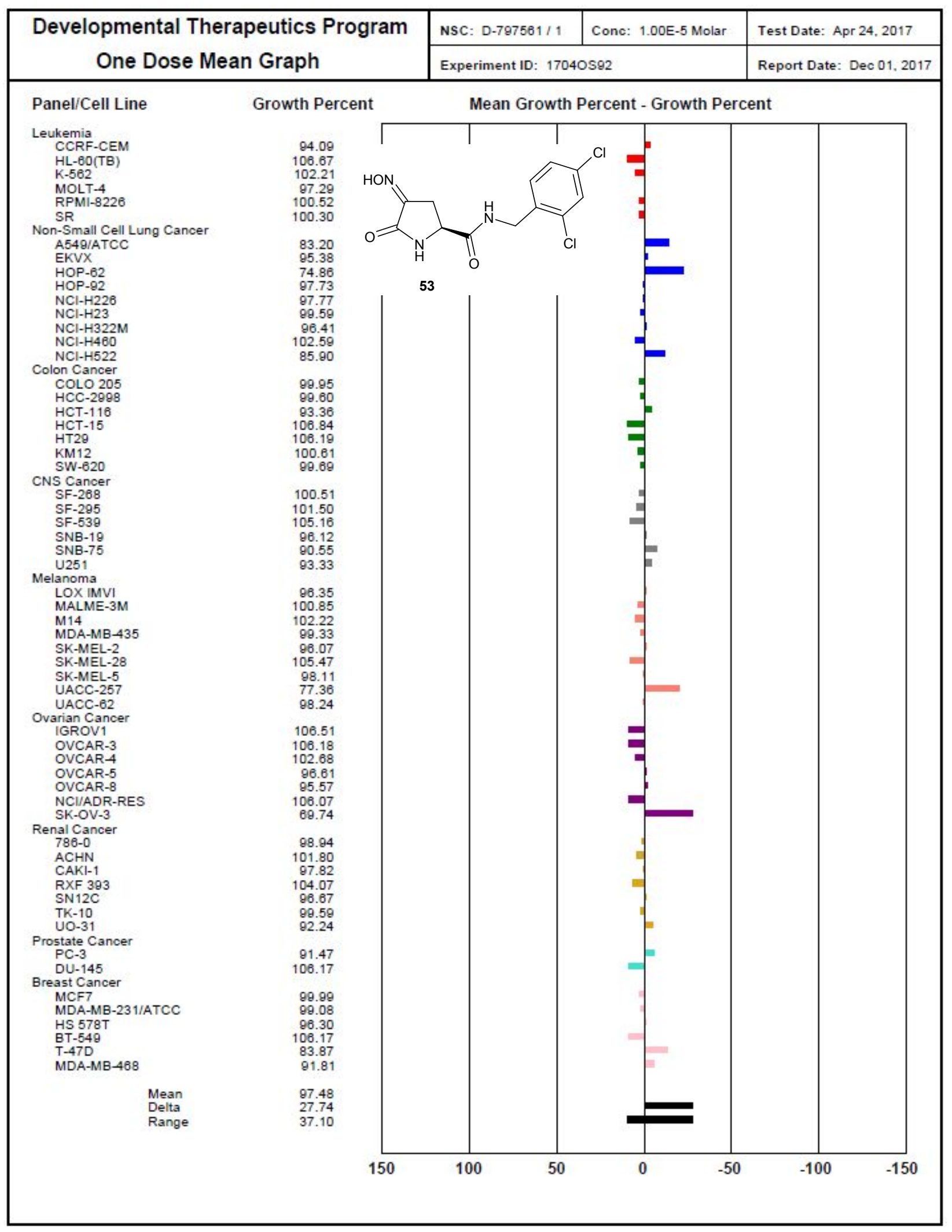




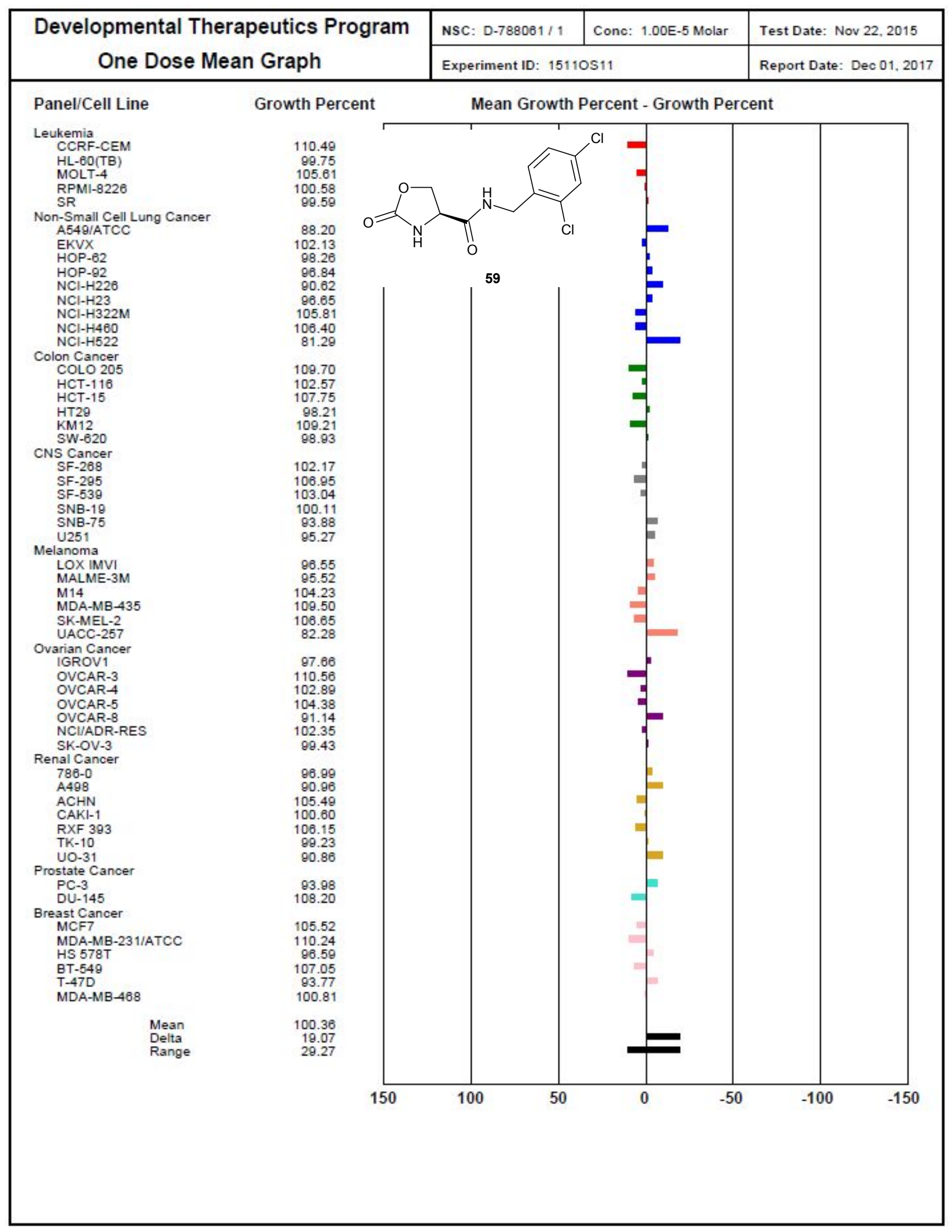




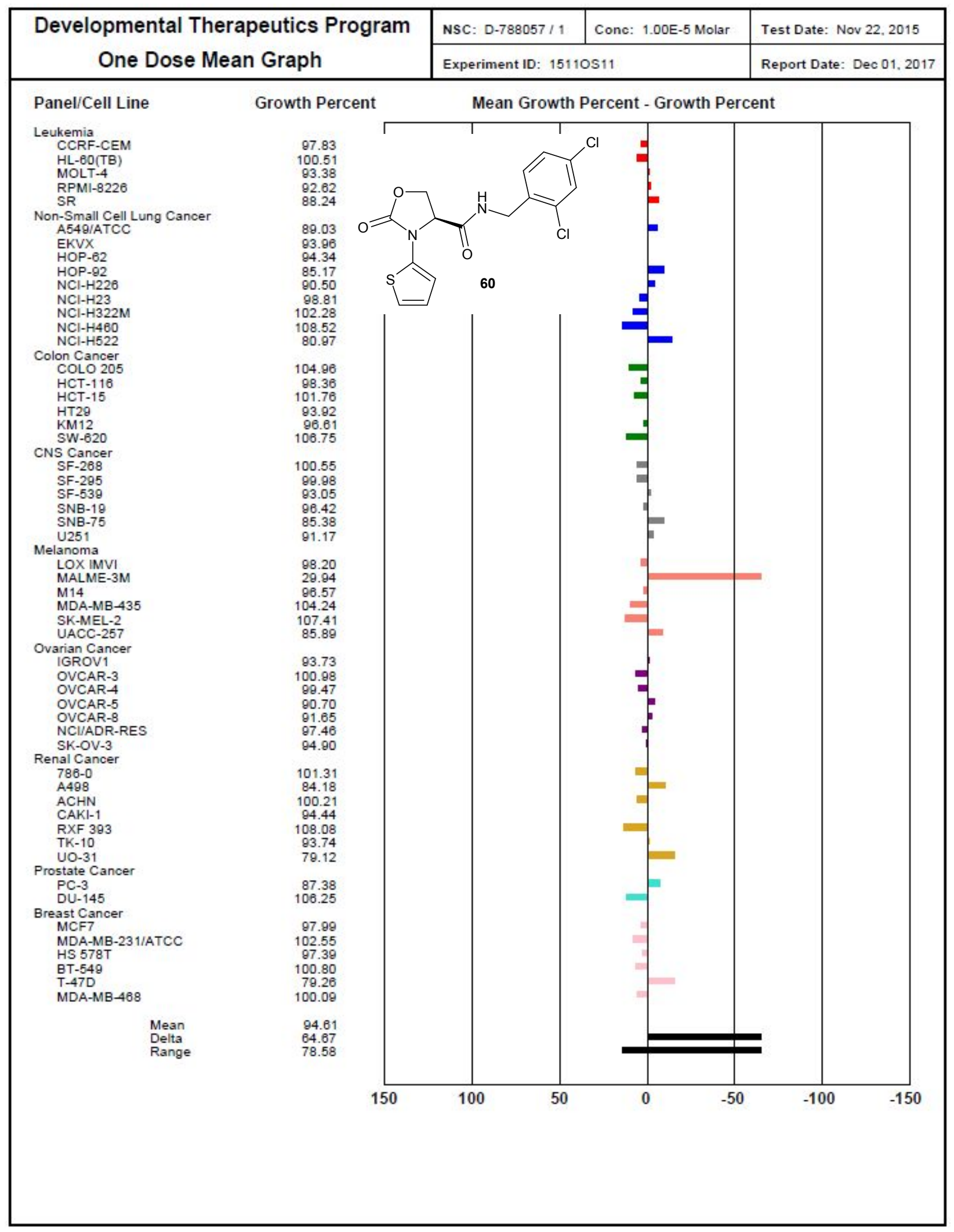




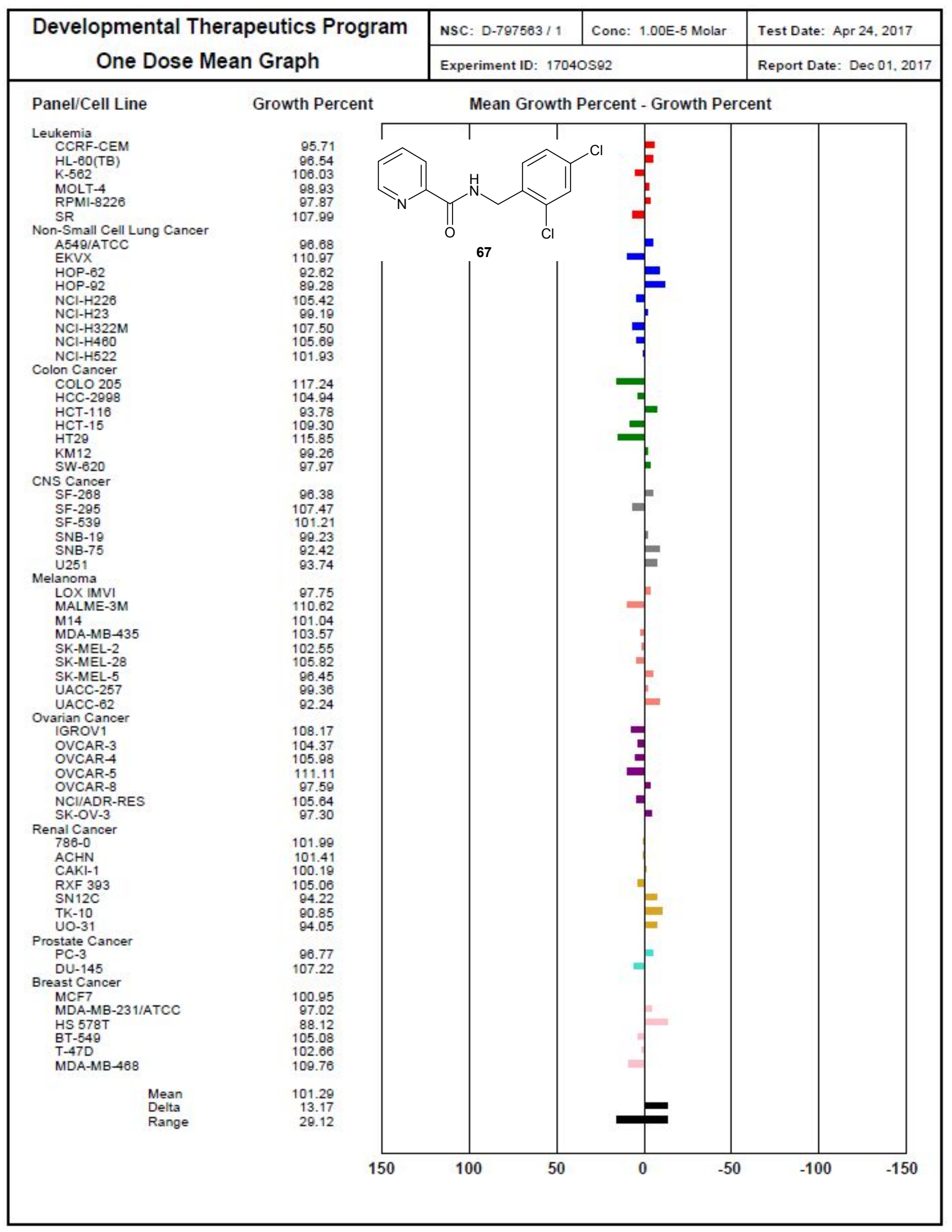




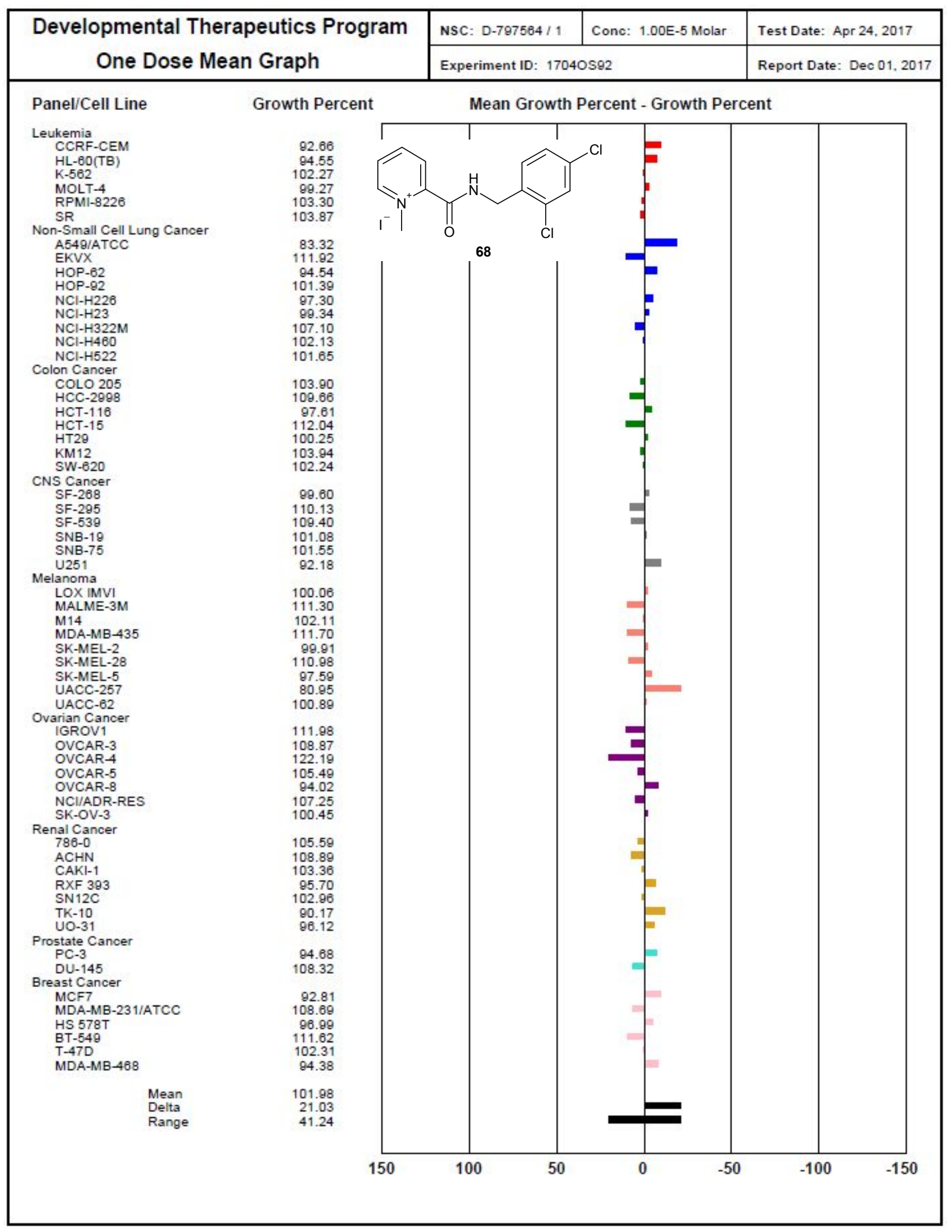




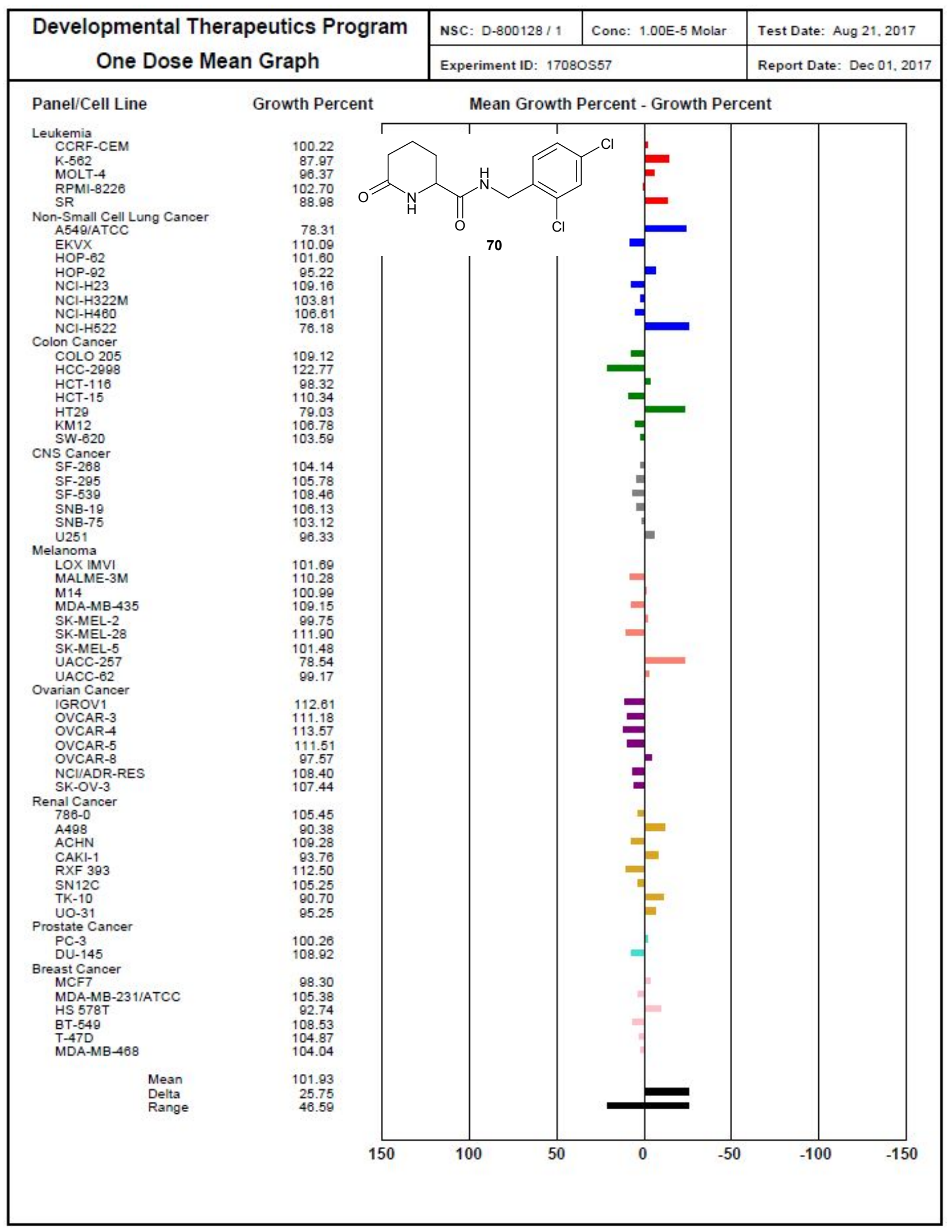


Figure S16. Flow cytometry analysis of macrophages, macrophages + LPS + BzATP, macrophages + LPS + BzATP + compound 1a, macrophages + LPS + BzATP + compound 16i and macrophages + LPS + BzATP + compound 16j

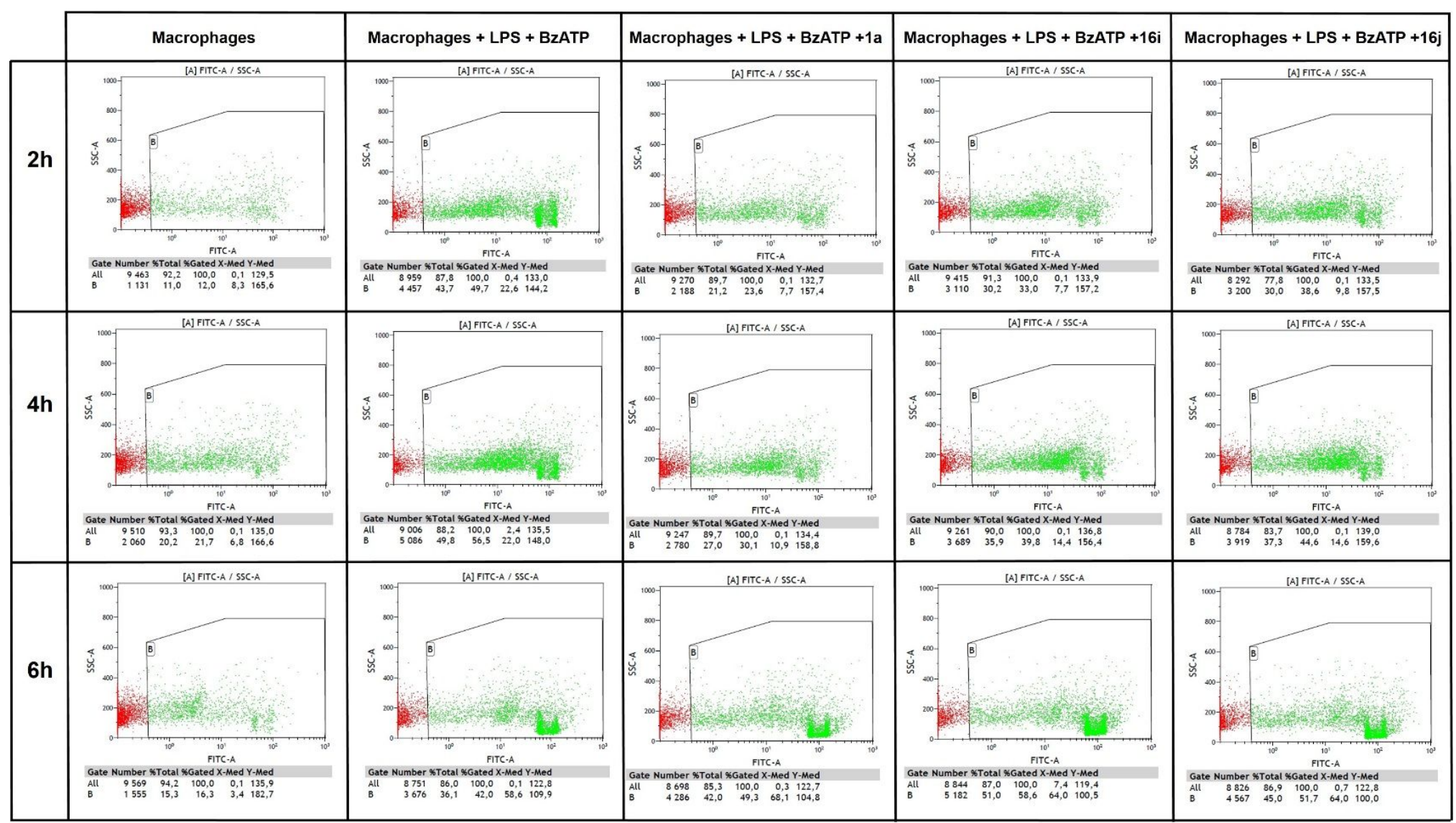




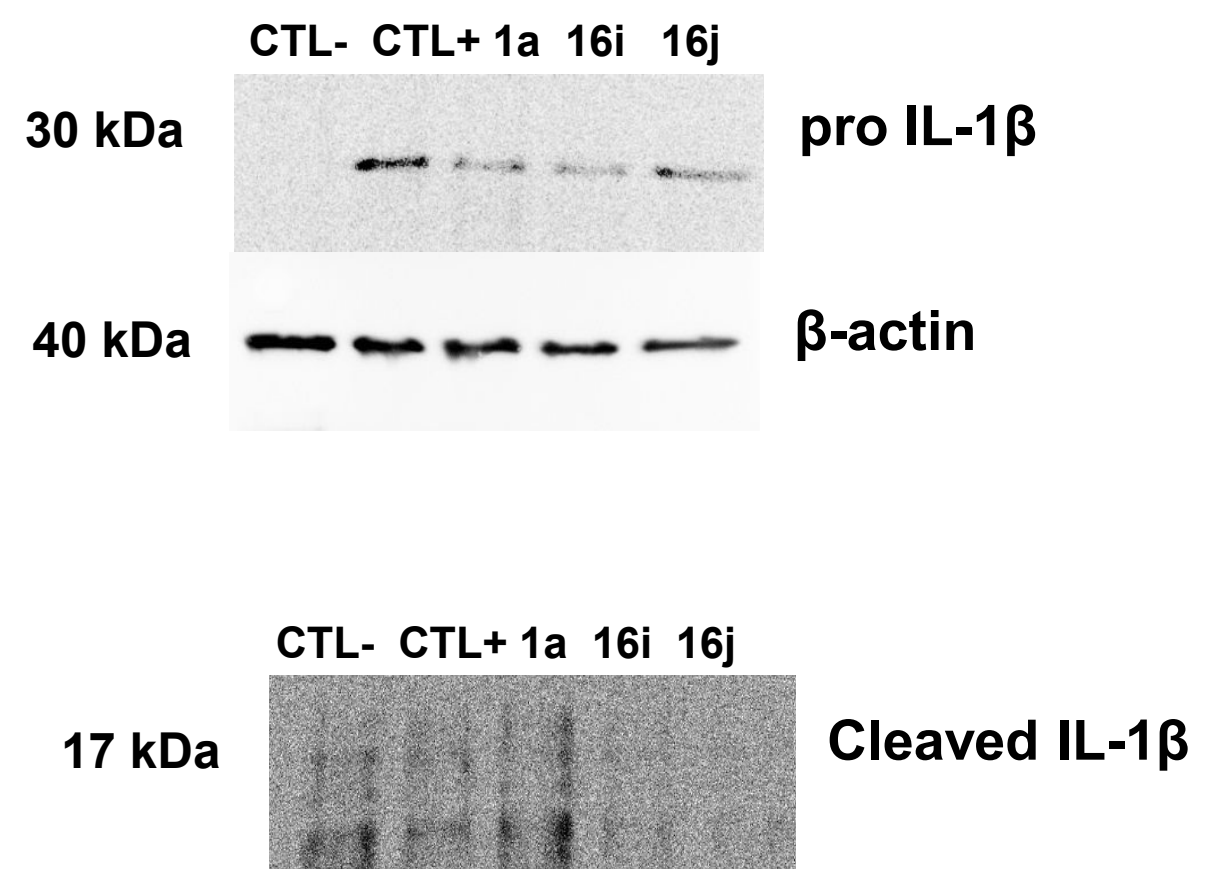

40 kDa

$\beta$-actin

\section{References}

1. Our previous work in the field of pyroglutamic acid derivatives: (a) Caulier, P.; Rigo, B.; Fasseur, D.; Couturier, D. Studies on pyrrolidones. Convenient syntheses of methyl, methyl $N$-methyl- and methyl $N$-methoxymethylpyroglutamate. J. Heterocycl. Chem. 1991, 28, 1143-1146. (b) Fasseur, D.; Rigo, B.; Leduc, C.; Caulier, P.; Couturier, D. Studies on pyrrolidones. Synthesis and $N$-alkylation of $\beta$-enaminoesters derived from pyroglutamic acid. J. Heterocycl. Chem. 1992, 29, 1285-1291. (c) Baudelet, D.; Daïch, A.; Rigo, B.; Lipka, E.; Gautret, P.; Homerin, G.; Claverie, C.; Rousseau, J.; Abuhaie, C.-M.; Ghinet, A. Impact of functional groups on the copper initiated N-arylation of 5functionalized pyrrolidin-2-ones and their vinylogues. Synthesis, 2016, 48, 2226-2244. (d) Homerin, G.; Baudelet, D.; Dufrénoy, P.; Rigo, B.; Lipka, L.; Dezitter, D.; Furman, C.; Millet, R.; Ghinet, A. $\mathrm{ZrCl}_{4}$ as a new catalyst for ester amidation: an efficient synthesis of $h$-P2X7R antagonists. Tetrahedron Lett., 2016, 57, 1165-1170. (e) Caulier, P.; Fasseur, D.; Couturier, D.; Rigo, B.; Kolocouris, A. Studies on pyrrolidones. On the carbamoylation of some pyroglutamic derivatives. $J$. Heterocycl. Chem., 1996, 33, 1233-1237. (f) Rigo, B.; Couturier, D. Studies on pyrrolidones. Synthesis of methyl N-(4-nitrobenzyl)pyroglutamate. J. Heterocycl. Chem., 1985, 22, 207-208. (g) Rigo, B.; Gautret, P.; Legrand, A.; Hénichart, J.-P.; Couturier, D. Reaction of trimethylsilyl benzhydryl ethers with methyl N-(trimethylsilyl)pyroglutamate: an easy and rapid $\mathrm{N}$-alkylation. Synlett, 1997, 998-1000. (h) Bourry, A.; Akué-Gédu, R.; Rigo, B.; Hénichart, J.-P.; Sanz, G.; Couturier, D. Studies on pyrrolidones. An improved Synthesis of $N$-arylmethyl pyroglutamic acids. $J$. 
Heterocycl. Chem., 2003, 40, 989-993. (i) Kolocouris, N. M.; Rigo, B. Etude dans la série des pyrrolidinones. Synthèse des acides N-(aryl methyl) oxo-5 pyrrolidine carboxyliques-2. Chim. Chron., 1982, 11, 309-317. (j) Homerin, G.; Lipka, E.; Rigo, B.; Millet, R.; Dezitter, D.; Furman, C.; Ghinet, A. Discovery of highly functionalized scaffolds: Pyrroloimidazolediones as P2X7 receptor antagonists. Tetrahedron 2017, 73, 5327-5336. (k) Homerin, G.; Lipka, E.; Rigo, B.; Farce, A.; Dubois, J.; Ghinet, A. On the discovery of new potent human farnesyltransferase inhibitors: emerging pyroglutamic derivatives. Org. Biomol. Chem., 2017, 15, 8110-8118.

2. Stanovnik, B.; Svete, J. Synthesis of Heterocycles from Alkyl 3-(Dimethylamino)propenoates and Related Enaminones. Chem. Rev. 2004, 104, 2433-2480.

3. Seki, M.; Hatsuda, M.; Mori, Y.; Yoshida, S.-I.; Yamada, S.-I.; Shimizu, T. A practical synthesis of (+)-biotin from L-cysteine. Chem. Eur. J., 2004, 10, 6102-6110.

4. Paytash, P. L.; Sparrow, E.; Gathe, J. C. The Reaction of Itaconic Acid with Primary Amines, J. Am. Chem. Soc., 1950, 72, 1415-1416.

5. Donnelly-Roberts, D. L.; Namovic, M. T.; Surber, B.; Vaidyanathan, S. X.; Perez-Medrano, A.; Wang, Y.; Carroll, W. A.; Jarvis, M. F. [(3)H]A-804598 ([(3)H]2-cyano-1-[(1S)-1-phenylethyl]-3quinolin-5-ylguanidine) is a novel, potent, and selective antagonist radioligand for $\mathrm{P} 2 \mathrm{X} 7$ receptors. Neuropharmacol., 2008, 56, 223-229.

6. Compound 74b was tritiated by Pharmaron UK Ltd. - The Old Glassworks, Nettlefold Road, Cardiff, CF24 5JQ, United Kingdom.

7. Chambers, L. J.; Gleave, R.; Senger, S.; Walter, D. S. WO 2008003697A1 (2008) Chem. Abstr. 2008, 148, 145026 .

8. Abdi, M. H.; Beswick, P. J.; Billinton, A.; Chambers, L. J.; Charlton, A.; Collins, S. D.; Collis, K. L.; Dean, D. K.; Fonfria, E.; Gleave, R. J.; Lejeune, C. L.; Livermore, D. G.; Medhurst, S. J.; Michel, A. D.; Moses, A. P.; Page, L.; Patel, S.; Roman, S. A.; Senger, S.; Slingsby, B.; Steadman, J. G. A.; Stevens, A. J.; Walter, D. S. Bioorg. Med. Chem. Lett. 2010, 20, 5080.

9. Homerin, G.; Baudelet, D.; Dufrenoy, P.; Rigo, B.; Lipka, E.; Dezitter, X.; Furman, C.; Millet, R.; Ghinet, A. Tetrahedron Lett. 2016, 57, 1165.

10.Cauliez, P.; Rigo, B.; Fasseur, D.; Couturier, D. J. Heterocycl. Chem., 1991, 28, 1143.

11.Nagasaka, T.; Tsukada, A.; Hamaguchi, F. Heterocycles, 1986, 24, 2015.

12.Fasseur, D.; Rigo, B.; Leduc, C.; Cauliez, P.; Couturier, D. J. Heterocycl. Chem., 1992, 29, 1285.

13.Baudelet, D.; Daich, A.; Rigo, B.; Lipka, E.; Gautret, P.; Homerin, G.; Claverie, C.; Rousseau, J.; Abuhaie, C.-M.; Ghinet, A. Synthesis 2016, 48, 2226.

14.Nebolsin, V. E.; Kromova, T. A. WO 2015137846 A1 (2015) Chem. Abstr. 2015, 163, 450241.

15.Koenig, W.; Geiger, R.; Wissmann, H.; Kruse, H. J.; Peterfalvi, M. DE 2423390 A1 19751204 (1975) Chem. Abstr. 1975, 84, 122346.

16.Homerin, G.; Lipka, E.; Rigo, B.; Millet, R.; Dezitter, D.; Furman, C.; Ghinet, A. Tetrahedron, 2017, 73, 5327.

17. Conde, S.; Lopez-Serrano, P.; Martinez, A. J. Mol. Catal. B: Enzym. 1999, 7, 299.

18. Brunet, M. A.; Boucherle, A.; Badinand, A. Chim. Therap. 1996, 3, 158.

19. Ellis, T. K.; Ueki, H.; Tiwari, R.; Soloshonok, V. A. Tetrahedron: Asymmetry 2009, $20,2629$.

20. Brunel, S.; Brumas, V.; Fiallo, M. M. L. Inorg. Chim. Acta 2009, 342, 4043.

21. Koehn, U.; Schramm, A.; Kloss, F.; Goerls, H.; Arnold, E.; Anders, E. Tetrahedron: Asymmetry 2007, 18, 1735 . 
22. Rigo, B.; Dolaine, R; El Ghammarti, S.; Couturier, D. J. Heterocycl. Chem., 1996, 33, 1063.

23. Rigo, B.; Gautret, P.; Legrand, A.; Hénichart, J.-P.; Couturier, D. Synlett, 1997, 998.

24. Rigo, B.; Erb, B.; El Ghammarti, S.; Gautret, P. J. Heterocycl. Chem., 1995, 32, 1599.

25. Homerin, G; Lipka, E.; Rigo, B.; Farce, A.; Dubois, J.; Ghinet, A. Org. Biomol. Chem., 2017, 15, 8110.

26. Seki, M.; Hatsuda, M.; Mori, Y.; Yoshida, S.-I.; Yamada, S.-I.; Shimizu, T. Chem. Eur. J., 2004, 10, 6102.

27. Wu, Y.-H.; Feldkamp, R. F.; Corrigan, J. R.; Rhodes, H. J. J. Org. Chem., 1961, 26, 1524.

28. Trot, O.; Olson, A. J. J. Comput. Chem., 2010, 31, 455-461.

29.(a) Kintz, P.; Richeval, C.; Jamey, C.; Ameline, A.; Allorge, D.; Gaulier, J.-M.; Raul, J.-S. Detection of the designer benzodiazepine metizolam in urine and preliminary data on its metabolism. Drug Test. Anal., 2017, 9, 1026-1033. (b) Vacek, J.; Papoušková, B.; Vrba, J.; Zatloukalová, M.; Křen, V.; Ulrichová, J. LC/MS metabolic study on quercetin and taxifolin galloyl esters using human hepatocytes as toxicity and biotransformation in vitro cell model. J. Pharm. Biomed. Anal., 2013, 86, 135-142.

30.Byzova, T.V.; Plow, E. F. Networking in the hemostatic system. Integrin alphaiibbeta3 binds prothrombin and influences its activation. $J$ Biol Chem, 1997, 272, 27183-27188. 